\title{
The Impact of HII Regions on the Interstellar Medium of our Galaxy
}

Matteo Luisi

West Virginia University, maluisi@mix.wvu.edu

Follow this and additional works at: https://researchrepository.wvu.edu/etd

Part of the Stars, Interstellar Medium and the Galaxy Commons

\section{Recommended Citation}

Luisi, Matteo, "The Impact of HII Regions on the Interstellar Medium of our Galaxy" (2019). Graduate Theses, Dissertations, and Problem Reports. 3921.

https://researchrepository.wvu.edu/etd/3921

This Dissertation is protected by copyright and/or related rights. It has been brought to you by the The Research Repository @ WVU with permission from the rights-holder(s). You are free to use this Dissertation in any way that is permitted by the copyright and related rights legislation that applies to your use. For other uses you must obtain permission from the rights-holder(s) directly, unless additional rights are indicated by a Creative Commons license in the record and/ or on the work itself. This Dissertation has been accepted for inclusion in WVU Graduate Theses, Dissertations, and Problem Reports collection by an authorized administrator of The Research Repository @ WVU.

For more information, please contact researchrepository@mail.wvu.edu. 


\title{
The Impact of H II Regions on the Interstellar Medium of our Galaxy

\author{
Matteo Luisi
}

\author{
Dissertation submitted \\ to the Eberly College of Arts and Sciences \\ at West Virginia University
}

in partial fulfillment of the requirements for the degree of

Doctor of Philosophy

in

Physics

Loren Anderson, Ph.D., Chair

Dana Balser, Ph.D.

Paul Cassak, Ph.D.

Duncan Lorimer, Ph.D.

D.J. Pisano, Ph.D.

Department of Physics and Astronomy

Morgantown, West Virginia, USA

2019

Keywords: Physics, Astronomy, H II Region, Interstellar Medium

Copyright 2019 Matteo Luisi 
Abstract

\title{
The Impact of H II Regions on the Interstellar Medium of our Galaxy
}

\author{
Matteo Luisi
}

The interstellar medium (ISM) of galaxies like the Milky Way contains lowdensity diffuse ionized gas known as the warm ionized medium (WIM). The WIM is the last major component of the ISM to be studied at high spatial and spectral resolution, and therefore many of its fundamental properties remain unclear. In particular, the connection between HII regions, areas of ionized hydrogen surrounding OB stars, and the WIM is not fully understood. OB stars emit large amounts of ionizing radiation and it is believed that a fraction of this radiation escapes from their HII regions and into the ISM where it is responsible for maintaining the ionization of the WIM. The goal of this work is to better understand how the radiation produced by OB stars is able to leak from the H II regions, how the radiation field changes during this process, and how the radiation affects the ambient ISM.

Using Green Bank Telescope (GBT) radio recombination line (RRL) data of a subset of Galactic H II regions, we show that the morphology of the photodissociation region surrounding an H II region strongly affects the amount of leaking radiation. We compute a leaking photon fraction of $15 \pm 5 \%$ for the compact H II region NGC 7538 and argue that more luminous H II regions likely allow a greater fraction of ionizing radiation to escape. We also show that physically large H II regions affect the surrounding ISM out to larger distances from the region. As a result, giant $\mathrm{H}$ II region complexes may have a greater effect on maintaining the ionization of the WIM. The $N\left({ }^{4} \mathrm{He}^{+}\right) / N\left(\mathrm{H}^{+}\right)$abundance ratio decreases with distance from most observed regions, indicating that heliumionizing photons are being absorbed within the H II region ionization fronts.

We find that WIM emission toward the first Galactic quadrant is correlated both with the locations of luminous H II region complexes and with the intensity of diffuse $8 \mu \mathrm{m}$ emission caused by excitation of polycyclic aromatic hydrocarbons (PAHs). This suggests that the soft-ultraviolet photons required to excite PAHs have the same origin as the more energetic radiation maintaining the WIM.

The GBT Diffuse Ionized Gas Survey (GDIGS) is an ongoing GBT project to map the inner-Galaxy WIM in RRL emission. These observations will allow us to better understand the large-scale distribution and dynamical state of the WIM. With the GDIGS data, we will also be able to constrain the physical properties of the WIM and determine how leaking photons from H II regions affect the ISM across all spatial scales. 


\section{Acknowledgments}

I would first like to thank Loren Anderson for his support throughout my graduate studies. His guidance has made this a fun and rewarding journey. I cannot imagine a better advisor. I am also thankful for my dissertation committee of Dana Balser, Paul Cassak, Dunc Lorimer, and D.J. Pisano for their advice and careful reading of this manuscript.

A huge thank you goes to my wife Meg. She spent many hours listening to me talk about my research and she gave me encouragement whenever I needed it. And to George, who won my heart through countless puppy kisses, cuddles, and play sessions. I am not sure I could have completed this degree without them.

Thank you to my friends in Austria; I realize it is particularly rare to stay close after moving halfway across the world. And to the friends I made in Morgantown, who helped keep me sane and motivated during our many hikes and board game afternoons.

Finally, I would like to thank my family and, above all, my mom, for always sharing her optimism with me and for her unwavering support when I decided to move to the United States to go to graduate school. 


\section{Table of Contents}

List of Tables vi

List of Figures $\quad$ vii

1 Introduction 1

1.1 H

1.1.1 Structure and Physical Properties . . . . . . . . . . . 2

1.1.2 H II Region Evolution . . . . . . . . . . . . . . . . . . . 5

1.2 The Interstellar Medium . . . . . . . . . . . . . . . . . . . 6

1.2.1 Phases of the ISM . . . . . . . . . . . . . . 6

1.2.2 The Warm Ionized Medium . . . . . . . . . . . . . . . 7

1.2.3 The H in Region/WIM Connection . . . . . . . . . . . . . . 8

1.3 Basics of Radio Astronomy . . . . . . . . . . . . . . . . . . . . . 10

1.4 Emission Mechanisms of Ionized Gas . . . . . . . . . . . . . . . 12

1.4.1 Radio Continuum Emission . . . . . . . . . . . . . . . . . . 12

1.4.2 Radio Recombination Lines . . . . . . . . . . . . . . . . . 13

1.4.3 $\mathrm{H} \alpha$ Emission . . . . . . . . . . . . . . . . . . . 16

1.4.4 Infrared Emission . . . . . . . . . . . . . . . . . 16

1.5 Telescopes and Datasets Used . . . . . . . . . . . . . . . 17

1.5.1 Green Bank Telescope . . . . . . . . . . . . . . . . . . . . 17

1.5.2 Wide-field Infrared Survey Explorer . . . . . . . . . . . . . . . 19

1.5.3 Supplementary Data . . . . . . . . . . . . . . . . 19

1.6 Key Questions . . . . . . . . . . . . . . . . . . 20

1.7 Dissertation Outline . . . . . . . . . . . . . . . 20

2 H II Region Ionization of the Interstellar Medium: A Case Study of NGC 753822

2.1 Abstract . . . . . . . . . . . . . . . 22

2.2 Introduction . . . . . . . . . . . . . . . . . . . . . . . . . . . . . . . . . . . . . . .

2.3 Observations . . . . . . . . . . . . . . . . . . 26

2.3.1 Radio Continuum . . . . . . . . . . . . . . 27

2.3.2 Radio Recombination Lines . . . . . . . . . . . . . . . . 27

2.4 Ionized Gas in and around NGC 7538 . . . . . . . . . . . . . . . . . . 31

2.4.1 The PDR Boundary . . . . . . . . . . . . . . . . . 31

2.4.2 The Leaking Emission Fraction . . . . . . . . . . . . . . . 34

2.4.3 Ionic Abundance Ratio . . . . . . . . . . . . . . . . . . . 40

2.4 Carbon RRLs . . . . . . . . . . . . . . . . . . . 43

2.4.5 Electron Temperature ................ . . . . 43

2.4.6 Dust Properties . . . . . . . . . . . . . . . . . . . 46

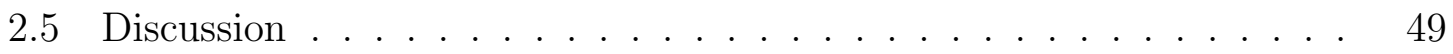

2.6 Summary . . . . . . . . . . . . . . . . . . . 52 
3 Diffuse Ionized Gas in the Milky Way Disk 54

3.1 Abstract . . . . . . . . . . . . . . . 54

3.2 Introduction . . . . . . . . . . . . . . . . . . 55

3.3 DIG RRL Emission . . . . . . . . . . . . . . . . . 57

3.4 Discussion . . . . . . . . . . . . . . . . . . . . . 58

3.4.1 The Galactic Location of the DIG . . . . . . . . . . . . . . 58

3.4.1.1 The $45 \mathrm{~km} \mathrm{~s}^{-1}$ Gas Component . . . . . . . . . 61

3.4.1.2 The $100 \mathrm{~km} \mathrm{~s}^{-1}$ Gas Component . . . . . . . . . . 65

3.4.1.3 Interacting Gas Clouds? . . . . . . . . . . . . 67

3.4.2 Intensity and Distribution of the DIG . . . . . . . . . . 70

3.4.2.1 The $45 \mathrm{~km} \mathrm{~s}^{-1}$ Gas Component . . . . . . . . . . 73

3.4.2.2 The $100 \mathrm{~km} \mathrm{~s}^{-1}$ Gas Component . . . . . . . . . 73

3.4.3 H I and the Diffuse Gas . . . . . . . . . . . . . . . . . . . . 75

3.4.4 Correlation with $8.0 \mu \mathrm{m}$ Intensity . . . . . . . . . . . . . . 77

3.5 Summary . . . . . . . . . . . . . . . . . . . . . 82

4 Ionization Profiles of Galactic H in Regions 84

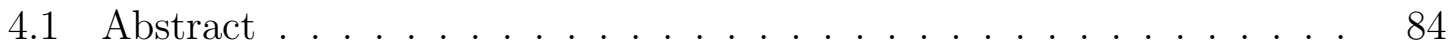

4.2 Introduction . . . . . . . . . . . . . . . . . . 84

4.3 Observations and Data Reduction . . . . . . . . . . . . . . . 89

4.4 The PDR Boundaries . . . . . . . . . . . . . . . . . . . . . . . . . . 92

4.5 Hydrogen RRL Emission . . . . . . . . . . . . . . . . . . . . . . 95

4.6 Ionic Abundance Ratios . . . . . . . . . . . . . . . . . . . . . . . . . 99

4.7 Physical Properties of the Ionized Gas . . . . . . . . . . . . . . . . . 103

4.7.1 LTE Electron Temperatures . . . . . . . . . . . . . . . . . . 103

4.7 .2 Electron Densities . . . . . . . . . . . . . . . . . . 105

4.7 .3 Non-LTE Analysis . . . . . . . . . . . . . . . . . . . 107

4.8 Line Profile Analysis . . . . . . . . . . . . . . . . . . . . . . . . . 109

4.9 Carbon RRLs . . . . . . . . . . . . . . . . . . . . . 110

$4.10 \mathrm{He}^{++}$Emission . . . . . . . . . . . . . . . . . . . . . 112

4.11 Conclusions . . . . . . . . . . . . . . . . . . . . . 114

5 Current and Future Plans 117

5.1 The GBT Diffuse Ionized Gas Survey . . . . . . . . . . . . . . . . 117

5.1 .1 Science Goals of GDIGS . . . . . . . . . . . . . . . . 118

5.1 .2 The Hir Region Complex W43 . . . . . . . . . . . . . . . . . 119

6 Conclusions $\quad 125$

6.1 Mechanisms of Ionizing Photon Escape . . . . . . . . . . . . . . . 125

6.2 Changes in the Radiation Field . . . . . . . . . . . . . . . . 126

6.3 The Impact of Leaking Photons on the ISM . . . . . . . . . . . . . 127

6.4 Large-Scale Properties of the WIM . . . . . . . . . . . . . . . 128

Appendix A Supplemental Data for Ionization Profiles of Galactic H II Regions 130 


\section{List of Tables}

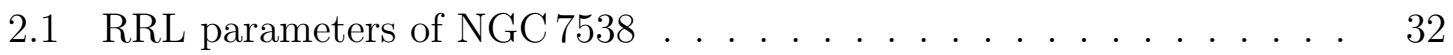

2.2 Derived Properties of NGC $7538 \ldots \ldots$. . . . . . . . . . . . . 41

3.1 RRL Emission Near W43 . . . . . . . . . . . . . . . . . 59

3.2 DIG Parameters . . . . . . . . . . . . . . . . 63

4.1 H II Region Properties . . . . . . . . . . . . . . . . . . . . . . . . . . 89

4.2 Fitted Hydrogen RRL Gradients . . . . . . . . . . . . . . . . . . . . 99

4.3 Fitted $y^{+}$Gradients and Spearman Coefficients . . . . . . . . . . 101

A.1 Observed Positions . . . . . . . . . . . . . . . . 130

A.2 H, He, and C RRL Parameters . . . . . . . . . . . . . . 134

A.3 Radio Continuum Temperatures and Source Properties . . . . . . . 156 


\section{List of Figures}

1.1 Examples of Galactic H II regions . . . . . . . . . . . . . . 3

1.2 Structure of a PDR . . . . . . . . . . . . . . . . 4

1.3 SED of an H II region . . . . . . . . . . . . . . . . . . 14

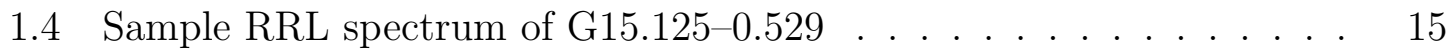

2.1 Radio continuum image of NGC $7538 \ldots \ldots \ldots \ldots$

2.2 RRL spectra of the 16 observed positions in NGC $7538 \ldots \ldots$. . . 30

2.3 WISE $12 \mu \mathrm{m}$ image of NGC $7538 \ldots \ldots \ldots \ldots \ldots$

2.4 WISE $12 \mu \mathrm{m}$ intensity convolved with the GBT beam . . . . . . 36

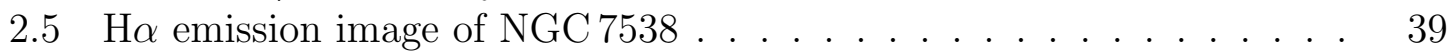

2.6 Ionic abundance ratio as a function of distance from NGC $7538 \ldots \ldots 42$

2.7 Carbon line intensity as a function of distance from NGC 7538 . . . 44

2.8 LTE electron temperature as a function of distance from NGC 7538 . 46

2.9 Dust temperature and column density map of NGC $7538 \ldots \ldots$. . . 48

2.10 RRL intensity as a function of distance from NGC 7538 and W43 . . 50

3.1 Velocity distribution of the DIG . . . . . . . . . . . . . 62

3.2 H I absorption spectra towards H II regions . . . . . . . . . . . 66

3.3 Distribution of RRL line widths . . . . . . . . . . . . . . . 69

3.4 Diffuse RRL Emission from $\ell=40^{\circ}$ to $18^{\circ} \ldots \ldots \ldots \ldots$

3.5 Longitude-velocity diagram of the DIG . . . . . . . . . . . 72

3.6 Correlation of the diffuse gas emission with Alves et al. (2015) . . . . 74

3.7 Correlation between $45 \mathrm{~km} \mathrm{~s}^{-1}$ and $100 \mathrm{~km} \mathrm{~s}^{-1}$ DIG intensities . . . . 76

3.8 Comparison of DIG RRL emission with H I and CO emission . . . . 78

3.9 Locations of multiple-velocity H II regions . . . . . . . . . . . . 79

3.10 Correlation between $8.0 \mu \mathrm{m}$ emission and the number of $\mathrm{HII}$ regions . 80

3.11 Correlation between $8.0 \mu \mathrm{m}$ emission and RRL emission . . . . . . . 81

4.1 Observed H II regions in $12 \mu \mathrm{m}$ WISE emission . . . . . . . . . . . 93

4.2 Hydrogen RRL intensities and ionic abundance ratios . . . . . . . 96

4.3 Hydrogen RRL intensities as a function of distance from the $\mathrm{H}$ II regions 98

4.4 Ionic abundance ratios . . . . . . . . . . . . . . . . . . 102

4.5 LTE electron temperatures . . . . . . . . . . . . . . . . . . . 104

4.6 Emission measure and electron density for each observed direction . . 106

4.7 Electron temperature derived from our line profile analysis . . . . . 111

4.8 Carbon line intensities . . . . . . . . . . . . . . . . . . . 113

5.1 GDIGS survey range $\ldots \ldots \ldots \ldots \ldots \ldots$

$5.2 \quad$ RRL moment 0 map of W43 . . . . . . . . . . . . . . . 120

5.3 WIM-only map of W43 . . . . . . . . . . . . . . . . . . 121

5.4 Leaking emission model . . . . . . . . . . . . . . . . . . . . 123

5.5 Longitude-velocity diagram of $\mathrm{W} 43 \ldots \ldots \ldots \ldots \ldots$

A.1 $\alpha$ RRL spectra of all observed positions . . . . . . . . . 160 
A.2 $\beta$ RRL spectra of all observed positions . . . . . . . . . . . . . 164

A.3 $\gamma$ RRL spectra of all observed positions . . . . . . . . . . 168 


\section{Chapter 1}

\section{Introduction}

\subsection{H II Regions}

The hottest and most massive stars, O- and B-type stars, emit enough ultraviolet (UV) photons to fully ionize their surroundings. The resulting volume of ionized hydrogen is known as an HII region (Ter Haar, 1946), following the astronomical practice of using the Roman numeral II to describe singly-ionized atoms. Because OB stars have lifetimes of only tens of megayears, H II regions are considered zero-age objects compared to the dynamical timescale of our Galaxy. Within the lifetime of the ionizing star, H II regions tend to not travel far $(\lesssim$ a few pc) from the environment in which they were born and they therefore trace ongoing high-mass star formation in the Milky Way.

H II regions were first detected at optical wavelengths. In a study of the Cygnus and Cepheus regions, Struve \& Elvey (1938) observed emission from ionized hydrogen and oxygen in the Galactic plane. They found that the observed emission decreases rapidly for high Galactic latitudes where high-mass star formation is uncommon. They therefore argued that the observed spectral lines were caused by the UV radiation of OB stars in the Galactic plane.

Because there is significant interstellar extinction at optical wavelengths, many subsequent observations of H II regions were performed at radio wavelengths at which the interstellar medium (ISM) is optically thin. Haddock et al. (1954) first observed radio continuum emission from the Orion nebula and M17 and Höglund \& Mezger (1965) reported on the first hydrogen radio recombination line (RRL; see §1.4.2) detections in a total of $11 \mathrm{H}$ II regions.

More recently, infrared (IR) data have played an important role in identifying H II region candidates. Wood \& Churchwell (1989) and Kurtz et al. (1994) used selection criteria based on Infrared Astronomical Satellite (IRAS) data to find ultra- 
compact (UC) H II region candidates. Using Wide-Field Infrared Survey Explorer (WISE) data, Anderson et al. (2014) compiled the WISE Catalog of Galactic H II Regions, a list of $\sim 8,500 \mathrm{HII}$ regions and $\mathrm{H}$ II region candidates. The H II Region Discovery Survey (HRDS; Bania et al., 2010) is based on similar IR criteria. HRDS observations showed that over $95 \%$ of IR candidates with radio continuum emission also have RRL emission and are thus bona fide H II regions. To date, the HRDS has discovered $\sim 900$ individual HII regions and more than doubled the previous H II region census (Anderson et al., 2011; Anderson et al., 2015a, 2018).

\subsubsection{Structure and Physical Properties}

The size of an H II region can be estimated by the Strömgren model (Strömgren, 1939) if the type of the ionizing star is known. For an HII region in ionization equilibrium, the volume ionization rate $I$ must be equal to the recombination rate $R$. Assuming that the $\mathrm{H}$ II region is entirely composed of hydrogen, the ionization rate

$$
I=\frac{3 N_{\mathrm{ly}}}{4 \pi R_{\mathrm{s}}^{3}},
$$

where $N_{\text {ly }}$ is the number of Lyman-continuum photons $(E>13.6 \mathrm{eV})$ emitted by the star per unit time and $R_{\mathrm{S}}$ is the Strömgren radius of the H II region. The recombination rate

$$
R=n_{\mathrm{e}}^{2} \alpha_{\mathrm{H}},
$$

where $n_{\mathrm{e}}$ is the electron number density and $\alpha_{\mathrm{H}}$ is the total recombination coefficient. Setting these two rates equal and solving for the Strömgren radius yields

$$
R_{\mathrm{s}}=\left(\frac{3}{4 \pi} \frac{N_{\mathrm{ly}}}{n_{\mathrm{e}}^{2} \alpha_{\mathrm{H}}}\right)^{1 / 3} .
$$

For an evolved H II region in ionization equilibrium, the Strömgren model is usually a good approximation despite the fact that real $\mathrm{H}$ II regions contain helium ( $25 \%)$ and heavier elements $(1-2 \%)$. In addition, H II regions form in inhomogeneous environments and their morphology is seldom that of a perfect sphere (see Figure 1.1). 

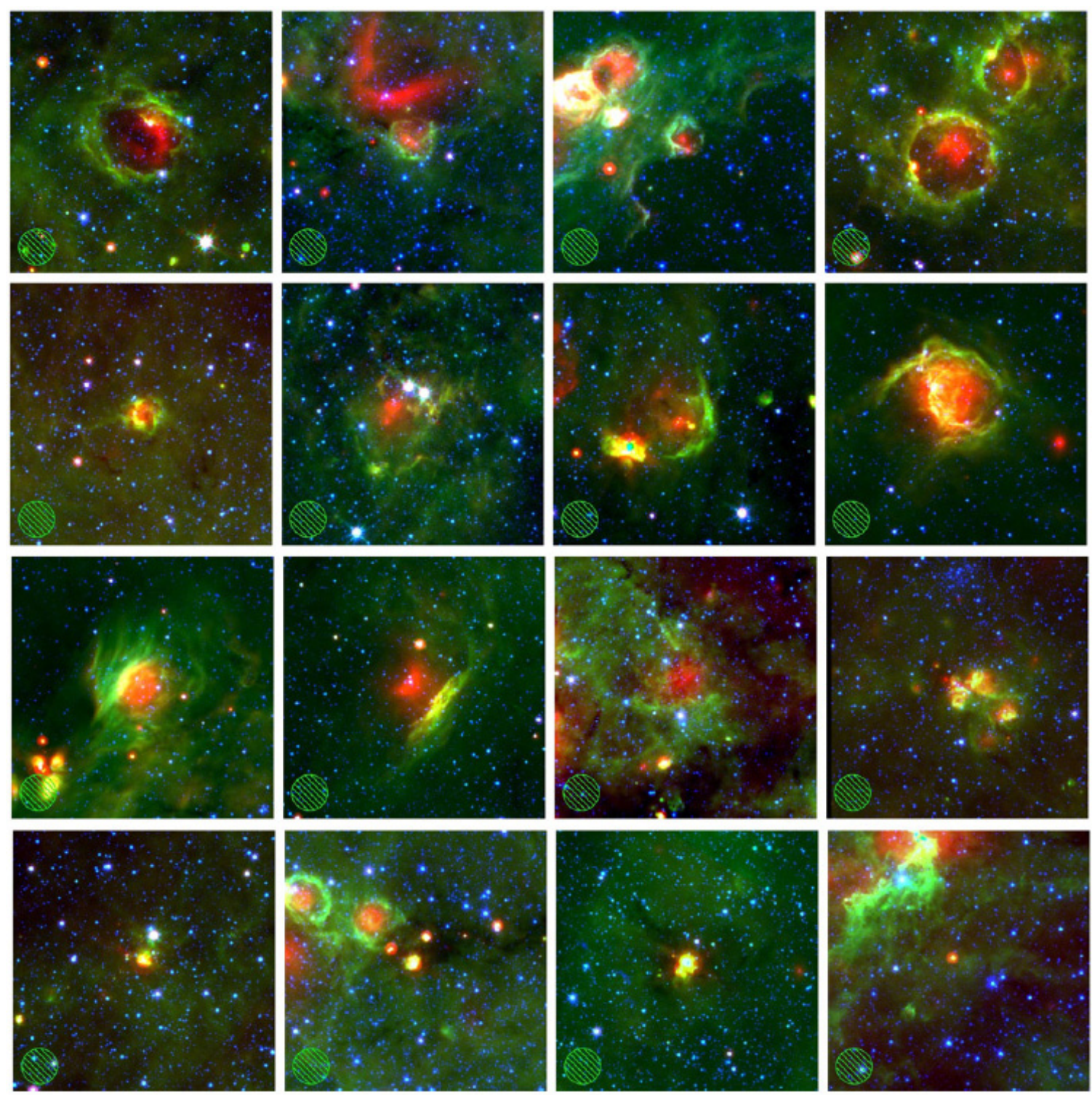

Figure 1.1: Examples of Galactic H II regions. Each panel shows a three-color image of an H II region: MIPSGAL $24 \mu \mathrm{m}$ (red), GLIMPSE $8.0 \mu \mathrm{m}$ (green), and GLIMPSE $3.6 \mu \mathrm{m}$ (blue). Images from the H II Region Discovery Survey (Anderson et al., 2011). 


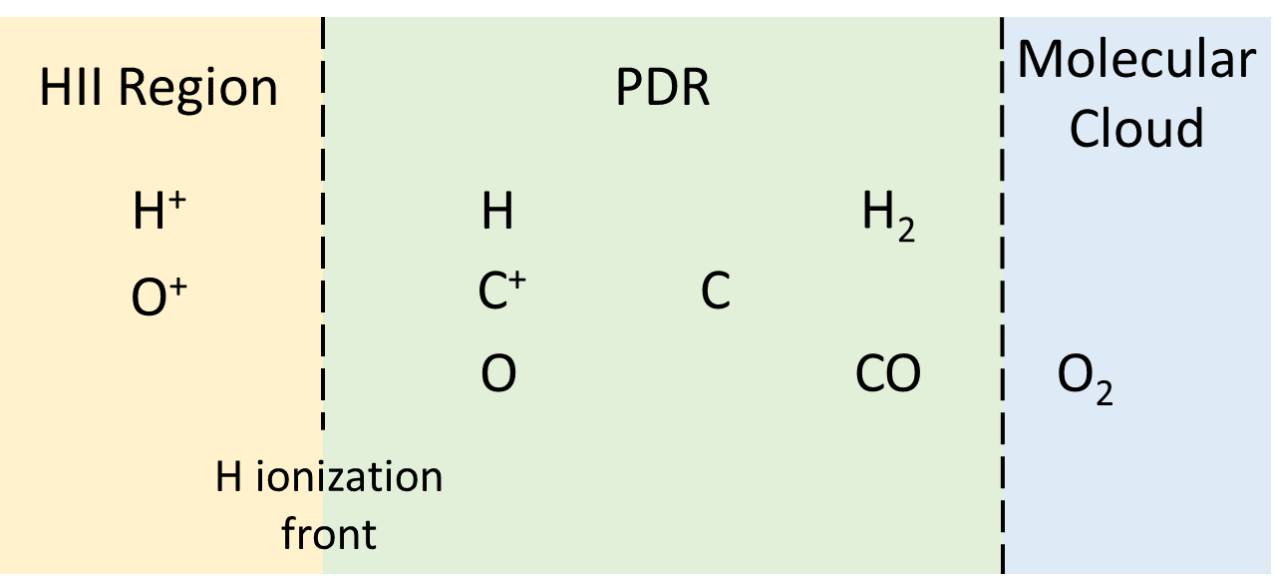

Figure 1.2: Structure of a PDR between an H II region (left) and a molecular cloud (right).

Due to density variations in the surrounding medium, the radiation emitted by the OB star will travel different distances depending on the direction. A significant fraction of the emitted radiation can escape from the H II region altogether. H II regions, for which the ionized region is determined by the absorption of ionizing photons, are known as ionization-bounded H II regions. Such regions are typically embedded in larger clouds of neutral or molecular gas. Density-bounded H II regions, on the other hand, do not contain enough matter to absorb all ionizing photons. These regions typically have much larger ionizing photon escape fractions than ionization-bounded H II regions.

On the interface between an H II region and the surrounding ISM lies the photodissociation region (PDR). PDRs are mostly neutral, however, their chemistry is strongly affected by UV photons emitted from the ionizing source. In particular, photons with energies below $13.6 \mathrm{eV}$ (the ionization potential of hydrogen) can ionize carbon within the PDR and dissociate various molecules, including $\mathrm{H}_{2}$. PDRs generally have distinct ionization and dissociation fronts for different species (see Figure 1.2). Like H II regions, PDRs are generally not homogeneous but instead have discontinuities and clumps. 


\subsubsection{H II Region Evolution}

H II regions can be found in various evolutionary states. Shortly after the birth of the ionizing star, an H II region is still strongly embedded within the molecular cloud. As the star ionizes the surrounding medium, the ionization front of the H II region rapidly expands until the region reaches the Strömgren radius. Once in ionization equilibrium, the region continues to expand, albeit more slowly. This phase is driven by the difference in pressure within the H II region and the ambient gas. The pressure differential is caused by two factors. First, the number of particles within the region roughly quadruples as the star ionizes the surrounding molecular hydrogen. Second, the temperature within the H II region is increased compared to the molecular cloud temperature by a factor of $10^{2}$ to $10^{3}$. The region continues to expand until its pressure is equal to that of its surroundings. Since the expansion speed is relatively slow (a few $\mathrm{kms}^{-1}$ ) and $\mathrm{OB}$ stars are short-lived, most $\mathrm{H}$ II regions are unable to achieve pressure equilibrium before the death of the ionizing source.

The physically smallest H II regions are usually also the youngest. These regions are known as UC or hyper-compact (HC) H II regions. Typical electron densities for UC H II regions are $\sim 10^{5} \mathrm{~cm}^{-3}$ and their physical sizes are $<0.1 \mathrm{pc}$. UC H II regions are deeply embedded within molecular clouds and thus difficult to observe at optical and IR wavelengths.

Compact and classical H II regions are at a later evolutionary state than UC H II regions. These regions have electron densities of $10^{2}$ to $10^{3} \mathrm{~cm}^{-3}$ and physical sizes of up to a few parsecs. Compact and classical H II regions have typically reached ionization equilibrium with their surroundings.

Diffuse H II regions are evolved, physically large (> 10 pc) H II regions with low electron densities $\left(<10 \mathrm{~cm}^{-3}\right)$. Due to their large sizes, diffuse H II regions may be among the most luminous H II regions in our Galaxy despite their low surface brightness (Anderson et al., 2018). Their PDRs are often weak or fragmented, suggesting that diffuse H II regions may leak a large fraction of ionizing photons into the ISM (see also $§ 1.2 .3)$. 


\subsection{The Interstellar Medium}

The ISM is defined as the matter that exists between the stars and other compact objects within our Galaxy. Stars are formed from the densest regions within the ISM and are recycled back into the ISM at the end of their lifetimes. The ISM therefore plays a crucial role in the chemical evolution of our Galaxy. Furthermore, the ISM affects most astronomical observations, either by selectively attenuating the observed signal or by various emission processes within the ISM itself.

By mass, the ISM consists of $\sim 99 \%$ gas and $\sim 1 \%$ dust. The gas is mostly hydrogen $(\sim 70 \%$ by mass) and helium $(\sim 30 \%)$, with a small fraction $(\sim 1 \%)$ of heavier elements. The density of the ISM varies from $\sim 10^{-3} \mathrm{~cm}^{-3}$ to $\sim 10^{6} \mathrm{~cm}^{-3}$, depending on the environment and the state of the gas. It is estimated that up to $\sim 10 \%$ of all baryons in the Milky Way are in the ISM, based on observations of diffuse neutral and molecular hydrogen (e.g., Kalberla \& Kerp, 2009).

\subsubsection{Phases of the ISM}

The ISM can be divided into three main phases: hot ionized gas, warm ionized or atomic gas, and cold atomic or molecular gas. The phases do not share the same volume in space (although they can transition from one phase into another) and they are typically in rough pressure equilibrium. Each phase is defined by characteristic temperatures and densities.

The hot ionized medium (HIM) is the most energetic phase, with temperatures of $\sim 10^{6} \mathrm{~K}$ and densities of $10^{-3} \mathrm{~cm}^{-3}$ to $10^{-2} \mathrm{~cm}^{-3}$. It is found within the Galactic halo where it makes up most of the baryonic matter. The HIM is often called the "X-ray corona" of the Milky Way, because of its bright soft X-ray emission and its large scale height of $1-3 \mathrm{kpc}$. It is believed that the HIM is heated by stellar wind driven bubbles and supernovae.

The warm gas can be divided into three sub-categories: dense ionized gas associated with discrete HII regions, diffuse ionized gas known as the warm ionized medium (WIM), and the warm neutral medium (WNM). The dense H II gas with 
temperatures of $\sim 10^{4} \mathrm{~K}$ surrounds OB stars (see also §1.1). The WIM has similar temperatures but pervades the Galactic disk and is not directly associated with any $\mathrm{H}$ II regions. With a scale height of $\sim 1 \mathrm{kpc}$ and number densities of $\sim 0.1 \mathrm{~cm}^{-3}$, the WIM is believed to be maintained by OB stars whose ionizing photons escape from the surrounding HII region and into the ISM (see §1.2.3). The WNM has slightly lower temperatures $(\sim 5,000 \mathrm{~K})$ than the WIM and consists of predominantly neutral atomic hydrogen and helium.

The cold gas exists in either atomic $(\mathrm{HI})$ or molecular form $\left(\mathrm{H}_{2}\right)$. The atomic "cold neutral medium" (CNM) is often associated with the molecular gas but has slightly higher temperatures $(\sim 100 \mathrm{~K})$ and lower densities $\left(\sim 30 \mathrm{~cm}^{-3}\right)$. The cold molecular gas forms dense clouds, which make up $<1 \%$ of the fractional volume of the ISM, but have particle densities up to $10^{6} \mathrm{~cm}^{-3}$ and contain a significant fraction ( $\sim 20 \%$ ) of the total ISM mass. Massive molecular clouds, for which the gas pressure is insufficient to support against their gravitational potential, eventually collapse and form stars. This process takes, on average, several million years for OB stars, a timespan comparable to the overall lifetime of the star.

\subsubsection{The Warm Ionized Medium}

The WIM is a major, widespread component of the ISM, with a scale height of $\sim 1 \mathrm{kpc}$, temperatures between 6,000 and 10,000 K (e.g., Reynolds, 1989; Haffner

et al., 2009), a number density of $\sim 0.1 \mathrm{~cm}^{-3}$, and an $n\left(\mathrm{H}^{+}\right) / n\left(\mathrm{H}^{0}\right)$ ionization ratio $\geq 13$ (Reynolds et al., 1998). There is evidence that the ionization fraction of the WIM is lower than that of H II regions and that the WIM is ionized by a softer radiation field (Madsen et al., 2006). With a smaller scale height of $\sim 100 \mathrm{pc}$, the "extendedlow density medium" (ELDM) is sometimes considered a separate diffuse ionized gas component of the ISM, despite having similar densities and electron temperatures as the WIM.

The existence of the WIM was first posited by Hoyle \& Ellis (1963) who found free-free emission (see §1.4.1) originating from the Galactic plane suggestive of a lowdensity layer of warm ionized hydrogen. Their measurements were later confirmed 
by Hewish et al. (1968) and Guélin (1974) who found that radio signals from pulsars showed dispersion measures consistent with the electron density derived by Hoyle \& Ellis (1963). Soon thereafter, optical emission lines originating from the WIM were detected (Reynolds, 1971; Reynolds et al., 1973). With the onset of CCD detectors, diffuse ionized $10^{4} \mathrm{~K}$ gas was also found in $\mathrm{H} \alpha$ images of nearby external galaxies (e.g., Dettmar, 1990).

In studies of external galaxies, the WIM is often referred to as the "diffuse ionized gas" (DIG). Although the properties of the diffuse gas can more easily be measured in the Milky Way, observations of external galaxies are necessary to understand the connection between the DIG and global galactic properties (e.g., star formation rates). In edge-on galaxies, many studies focus on the disk-halo interaction between star-forming regions in the galactic plane and the DIG extending into the halo (e.g., Mac Low \& Ferrara, 1999; Strickland et al., 2004). Face-on galaxies, on the other hand, provide an ideal test bed to study the spatial correlation between H II region complexes and the diffuse gas (e.g., Ferguson et al., 1996; Zurita et al., 2000, see also $§ 1.2 .3)$.

\subsubsection{The Hir Region/WIM Connection}

There is some debate as to how the WIM maintains its ionization. Radiation produced by supernova explosions contributes, however, it falls short of the total energy requirements given the mass and distribution of the WIM (Hoopes \& Walterbos, 2003). The only source capable of injecting enough energy into the WIM are OB stars. The vast majority of OB stars, however, is confined to the Galactic midplane. The emitted photons must therefore travel up to several kiloparsecs through the ISM to account for the distribution of the WIM. The mechanisms of how the photons can traverse across these distances are still poorly understood.

Previous studies have shown that supernovae and stellar winds may create cavities in the ISM through which radiation can propagate without being absorbed (e.g., Cox \& Smith, 1974), or that the ISM may have naturally occurring low-density pathways due to a fractal geometry (Ciardi et al., 2002). Theory and observations have 
suggested the existence of superbubble-, or chimney-like structures in starburst galaxies (e.g., Mac Low \& Ferrara, 1999; Cecil et al., 2002; Strickland et al., 2004). These chimneys are hot $\left(\sim 10^{6} \mathrm{~K}\right)$ and fully ionized, such that the probability of Lymancontinuum radiation being absorbed within the chimney is negligible. While chimneys have also been predicted for Milky Way-like galaxies (Norman \& Ikeuchi, 1989), only a small number have been found in observations of non-starburst edge-on galaxies (Howk \& Savage, 2000; Rossa et al., 2004) and our own Galaxy (Terebey et al., 2003). This may be due to difficulties in disentangling these structures along the line of sight.

If the WIM is maintained by photons emitted by OB stars, then emission from the WIM should be spatially related with the location of massive star-forming regions. Rossa \& Dettmar (2003) show that the far-IR flux ratio at $60 \mu \mathrm{m}$ and $100 \mu \mathrm{m}$, a tracer of the star formation rate, is strongly correlated with the presence of diffuse ionized gas. In a study of two galaxies, Ferguson et al. (1996) demonstrate that the surface brightness distribution tracing the locations of $\mathrm{O}$ stars is related with $\mathrm{H} \alpha$ emission from ionized gas. The main difficulty in such studies, however, is to separate between H II region emission and DIG emission. Based on their morphologies and luminosities, Zurita et al. (2000) compiled a catalog of H II regions in a sample of six face-on galaxies, which they used to create a census of the DIG emission by masking pixels coincident with H II regions. They also find a spatial correlation between DIG emission and HII regions, and show that this correlation is strongest for the most luminous H II regions.

H II regions surrounding OB stars must allow a significant amount of ionizing radiation to leak into the ISM in order to maintain the ionization of the WIM. Averaged over entire galaxies, photon escape fractions of $30 \%$ (Zurita et al., 2002) to $50 \%$ (Giammanco et al., 2005) are consistent with observations and models. For the Large and Small Magellanic Cloud, 40\% of ionizing photons were found to escape from individual H II regions (Pellegrini et al., 2012). There has never been a comprehensive study of H II region leaking fractions in the Milky Way, although Anderson et al. (2015b) estimate that the bubble H II region RCW 120 is leaking $25 \pm 10 \%$ of 
its emission beyond its PDR.

As radiation escapes from an H II region, its spectrum changes due to absorption and re-emission processes. He-ionizing photons with energies $>24.6 \mathrm{eV}$ are easily absorbed within the HII region since the ionization cross-section of helium is much larger than that of hydrogen. For $\mathrm{O}$ stars with low effective temperatures $(<35,000 \mathrm{~K})$, nearly the entire He-ionizing radiation is suppressed before reaching the ambient ISM (Weber et al., 2019). This has been indirectly confirmed by Madsen et al. (2006), who found that the $\mathrm{HeI} / \mathrm{H} \alpha$ line ratio is lower in the WIM than in H II regions. In addition, it has been shown in observations and simulations that the H-ionizing continuum hardens as photons escape from the region, presumably because photons with energies $\gtrsim 13.6 \mathrm{eV}$ (the ionization potential of hydrogen) are preferentially absorbed by neutral hydrogen (Osterbrock, 1989). Since the ionization cross-section is largest for photon energies equal to the ionization potential and decreases with increasing energy, photons with energies $\gg 13.6 \mathrm{eV}$ are less likely to ionize hydrogen. This results in a relative hardening of the emergent radiation field from an H II region at energies between the ionization potential of hydrogen and that of helium (Wood \& Mathis, 2004).

\subsection{Basics of Radio Astronomy}

Radio astronomy covers a frequency range of roughly $30 \mathrm{MHz}$ to $300 \mathrm{GHz}$. The fundamental observable is the specific intensity of a source $I_{\nu}$, which is equal to its surface brightness if the source is resolved (i.e. larger in angular size than the telescope beam). In this case, the brightness does not depend on the distance to the source. For observations of astronomical sources, the specific intensity measured at frequency $\nu$ depends on the intensity of the background $I_{\nu, 0}$ plus the emission along the path $I_{\nu, \text { path}}$, both of which are attenuated by material along the line of sight,

$$
I_{\nu}\left(\tau_{\nu}\right)=I_{\nu, 0}\left(\tau_{\nu}\right) e^{-\tau_{\nu}}+I_{\nu, \operatorname{path}}\left(1-e^{-\tau_{\nu}}\right),
$$


where $\tau_{\nu}$ is the optical depth. The total intensity of a source is the specific intensity integrated over all frequencies,

$$
I=\int_{0}^{\infty} I_{\nu} d \nu
$$

In local thermodynamic equilibrium (LTE), $I_{\nu}$ is equal to the Planck function of a blackbody at temperature $T$,

$$
B_{\nu}=\frac{2 h \nu^{3}}{c^{2}} \frac{1}{e^{h \nu / k T}-1},
$$

where $h=6.626 \times 10^{-27} \mathrm{~cm}^{2} \mathrm{~g} \mathrm{~s}^{-1}$ is the Planck constant, $c=2.998 \times 10^{10} \mathrm{~cm} \mathrm{~s}^{-1}$ is the speed of light, and $k=1.381 \times 10^{-16} \mathrm{~cm}^{2} \mathrm{~g} \mathrm{~s}^{-2} \mathrm{~K}^{-1}$ is the Boltzmann constant. In radio astronomy where $h \nu \ll k T, B_{\nu}$ can often be approximated by the RayleighJeans limit of Eq. 1.6. In this case, it is straightforward to show that

$$
B_{\nu}=\frac{2 \nu^{2} k T}{c^{2}}
$$

For unresolved sources the specific intensity cannot be measured directly. In this case it is more useful to use flux density to describe the source. The flux density $S_{\nu}$ is defined as the specific intensity integrated over the source. With this definition,

$$
S_{\nu}=\int I_{\nu} \cos \theta d \Omega
$$

where $\Omega$ is the solid angle of the source and $\theta$ is the source angle in spherical coordinates. Most sources have angular sizes small enough that $\cos \theta \approx 1$. The unit of flux density is erg $\mathrm{cm}^{-2} \mathrm{~s}^{-1} \mathrm{~Hz}^{-1}$. Since astronomical sources are faint, it is common to use the unit Jansky (Jy) instead, where $1 \mathrm{Jy}=10^{-23} \mathrm{erg} \mathrm{cm}^{-2} \mathrm{~s}^{-1} \mathrm{~Hz}^{-1}$.

Both intensity and flux depend on the distance to the source. The corresponding intrinsic property of the source is the luminosity. The specific luminosity $L_{\nu}$ is defined as the total power per unit bandwidth radiated by an isotropic source at frequency $\nu$. In the local universe, it is related to the flux density by

$$
L_{\nu}=4 \pi D^{2} S_{\nu}
$$


where $D$ is the distance to the source. The unit of specific luminosity is $\operatorname{erg~s}^{-1} \mathrm{~Hz}^{-1}$, and $\operatorname{erg~s}^{-1}$ for the total luminosity. For OB stars, the total ionizing luminosity is often defined as the number of Lyman-continuum photons emitted per second.

Radio telescopes do not directly measure any of the above quantities. Instead, they register the total detected power from a source $P_{\nu}$, which depends on the flux density of the source and the physical properties of the telescope. For a point source of flux density $S_{\nu}$, a radio telescope measures the so-called antenna temperature

$$
T_{\mathrm{A}}=\frac{P_{\nu}}{k}=\frac{A_{\mathrm{e}} S_{\nu}}{2 k}
$$

Here, $A_{\mathrm{e}}$ is the effective collecting area of the telescope. It is often more convenient to use the main beam brightness temperature which is defined as $T_{\mathrm{MB}}=T_{\mathrm{A}} / \eta_{\mathrm{MB}}$, where $\eta_{\mathrm{MB}}$ is the main beam efficiency. In the Rayleigh-Jeans limit, Eq. 1.8 can be rewritten as

$$
S_{\nu}=\frac{2 k \nu^{2}}{c^{2}} \int T_{\mathrm{MB}} d \Omega_{\mathrm{MB}}
$$

where $\Omega_{\mathrm{MB}}$ is the solid angle of the telescope main beam size.

\subsection{Emission Mechanisms of Ionized Gas}

Ionized gas emits across the electromagnetic spectrum in both continuum and spectral lines. The basic emission mechanisms are the same for dense ionized gas found within H II regions and the diffuse WIM. Given below is a discussion of radio continuum and RRL emission (which constitute the bulk of observations in this work), $\mathrm{H} \alpha$ emission (an important optical tracer of ionized hydrogen), and various emission processes in the infrared regime.

\subsubsection{Radio Continuum Emission}

Continuum emission, the strongest emission mechanism at radio wavelengths, is due to thermal bremsstrahlung (free-free radiation). As free electrons interact with ions, they are decelerated and lose kinetic energy in the process, which is converted 
into radiation. Since the change in energy can take any value up to a maximum of the initial kinetic energy of the electron, free-free radiation has a continuous spectrum.

The spectral energy distribution (SED) of free-free radiation is steep for optically thick emission (with slope $\sim 2$ ), turns over where the emission transitions from optically thick to optically thin, and has only a weak dependence on frequency in the optically thin case (with slope $\sim-0.1$; see Figure 1.3). The turnover frequency $\nu_{0}$ depends on the temperature and the emission measure. As shown by Anderson (2010),

$$
\nu_{0}=304.5 \mathrm{MHz}\left(\frac{T_{\mathrm{e}}}{\mathrm{K}}\right)^{-0.643}\left(\frac{\mathrm{EM}}{\mathrm{cm}^{-6} \mathrm{pc}}\right)^{0.476},
$$

where $T_{\mathrm{e}}$ is the electron temperature and the emission measure,

$$
\mathrm{EM}=\int_{0}^{s} n_{\mathrm{e}}^{2} d s
$$

where $n_{\mathrm{e}}$ is the electron number density and $s$ is the observed path length along the line of sight. For H II regions, the turnover frequency is on order of $1 \mathrm{GHz}$ and it is lower for diffuse ionized gas.

\subsubsection{Radio Recombination Lines}

After an ion and an electron recombine, the electron cascades from highly excited states to lower energy states. Each time the electron drops to a lower energy level, radiation with a characteristic rest frequency $\nu_{0}=E / h$ is emitted, where $E$ is the difference between energy levels. The frequency can also be expressed as a function of the electron's principal quantum number, such that

$$
\nu_{0}=R_{\infty} c\left(1+\frac{m_{\mathrm{e}}}{M}\right)^{-1}\left[\frac{1}{n^{2}}-\frac{1}{(n+\Delta n)^{2}}\right]
$$

where $R_{\infty}=1.097 \times 10^{5} \mathrm{~cm}^{-1}$ is the Rydberg constant, $m_{\mathrm{e}}=9.109 \times 10^{-28} \mathrm{~g}$ is the mass of an electron, $M$ is the mass of the nucleus, $n$ is the final electronic level, and $\Delta n$ is the change in level. Recombination lines for which $\nu_{0}$ is in the radio regime are known as RRLs. Shown in Figure 1.4 is a sample RRL spectrum of a Galactic H II 


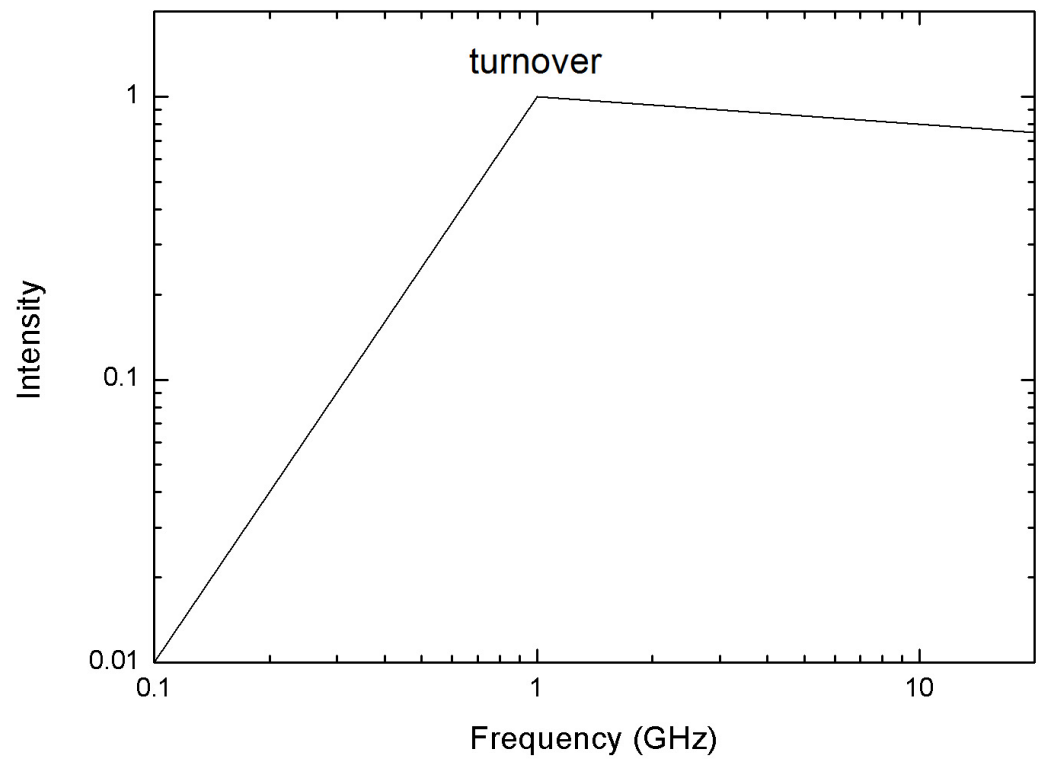

Figure 1.3: Expected SED of an H II region. The spectrum turns over at the transition from optically thick to optically thin emission. The intensity units are arbitrary.

region.

The nomenclature for recombination lines is $\mathrm{X} n[\alpha / \beta / \gamma / \ldots]$, where $\mathrm{X}$ is the element, $n$ is the electronic level, and $\alpha / \beta / \gamma / \ldots$ is the change in level, where $\alpha$ corresponds to $\Delta n=1, \beta$ is $\Delta n=2$, etc. (Lilley et al., 1966). For example, H100 $\alpha$ is the hydrogen RRL transition from $n=101$ to $n=100$ at $6478.76 \mathrm{MHz}$.

RRLs are useful tools to study ionized gas because they have well-defined rest frequencies. Any deviation between the rest frequency of the emitted line and its observed frequency is due to the Doppler effect caused by the motion of the source along the line of sight. The line-of-sight velocity of the source,

$$
V=c \frac{\nu_{0}-\nu}{\nu_{0}}
$$

where $\nu$ is the observed line frequency. Velocities are defined as positive if the source is redshifted, i.e., it is moving away from us, and negative for blueshifted sources moving toward us. 


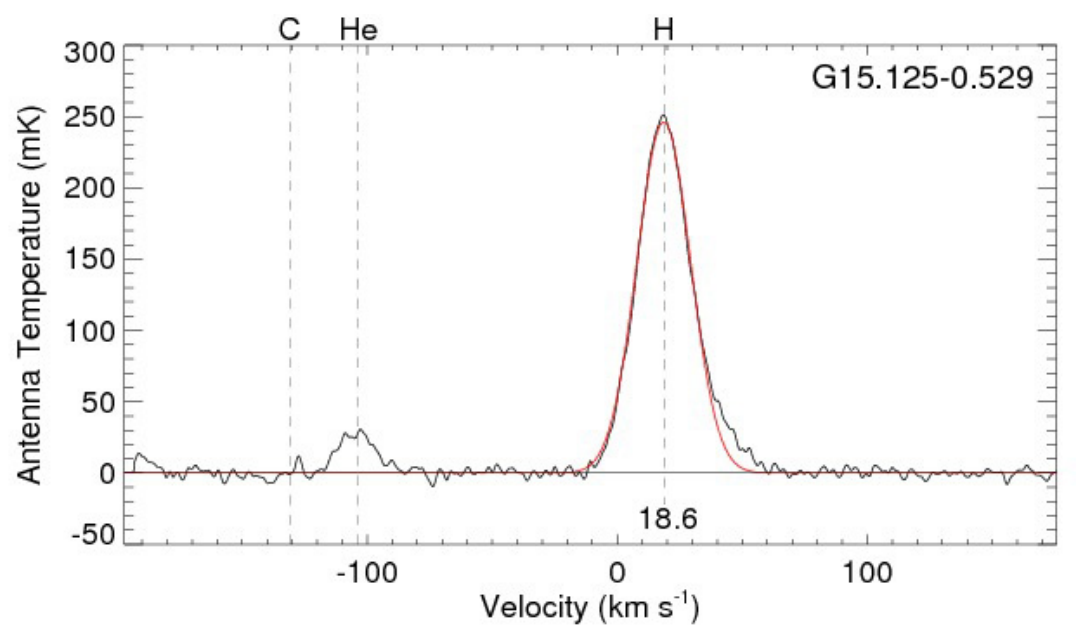

Figure 1.4: RRL spectrum for the Galactic H II region G15.125-0.529. The strongest line is hydrogen but emission from helium and carbon is also visible. The velocities for helium and carbon are shifted from that of hydrogen by $-122.15 \mathrm{~km} \mathrm{~s}^{-1}$ and $-149.56 \mathrm{~km} \mathrm{~s}^{-1}$, respectively, due to their larger atomic masses. The spectrum is an average of seven $\mathrm{H} n \alpha$ transitions at X-band $(8-10 \mathrm{GHz})$. Figure from the HRDS (Bania et al., 2010).

It is important to note that the movement of Earth has a strong effect on observed velocities. In Galactic astronomy, velocities are typically measured using the Local Standard of Rest (LSR). LSR velocities are corrected for the rotation of the Earth, Earth's motion relative to the Sun, and the motion of the Sun with respect to the local surrounding matter. For sources outside the Solar circle, measured LSR velocities can be converted into distances by assuming a model of the rotation of the Galaxy, and by assuming that the source does not move with respect to its ambient medium. For sources within the Solar circle, each velocity can be mapped to two possible distances. This effect is known as the kinematic distance ambiguity (KDA).

For optically thin lines, the RRL intensity of an extended source can be approximated by

$$
\left(\frac{T_{\mathrm{L}}}{\mathrm{K}}\right)=1.92 \times 10^{3}\left(\frac{T_{\mathrm{e}}}{\mathrm{K}}\right)^{-3 / 2}\left(\frac{\mathrm{EM}}{\mathrm{pc} \mathrm{cm}^{-6}}\right)\left(\frac{\Delta \nu}{\mathrm{kHz}}\right)^{-1}(\Delta n)^{-2},
$$

where $\Delta \nu$ is the full width at half-maximum (FWHM) of the line (Condon \& Ransom, 2016). In the radio regime, the FWHM line width is mainly a function of temperature 
and turbulence. The thermal line width for particles following a Maxwell-Boltzmann distribution,

$$
\mathrm{FWHM}=\sqrt{8 \ln 2} \sigma=2.355 \sigma
$$

where the one-dimensional velocity dispersion,

$$
\sigma=\left(\frac{k T_{\mathrm{e}}}{m}\right)^{0.5}
$$

For hydrogen clouds with $T_{\mathrm{e}}=10^{4} \mathrm{~K}$ the thermal line width is $\sim 20 \mathrm{~km} \mathrm{~s}^{-1}$. The turbulent line width is roughly equal to the thermal line width for H II regions and the WIM. Since RRLs have Gaussian line profiles, these components must be added in quadrature; typical hydrogen line FWHM values are therefore $\sim 30 \mathrm{~km} \mathrm{~s}^{-1}$.

Although RRLs are faint compared to recombination lines at higher frequencies, they have the benefit of being essentially free from the effects of extinction, a combination of absorption and scattering due to dust particles. Interstellar extinction is strongly dependent on wavelength and the line of sight. It is generally negligible at radio frequencies.

\subsection{3 $\mathrm{H} \alpha$ Emission}

$\mathrm{H} \alpha$ is a specific optical recombination line $(n=3$ to $n=2)$ with a rest wavelength of $656.47 \mathrm{~nm}\left(\nu_{0}=456.673 \mathrm{THz}\right) . \mathrm{H} \alpha$ emission is very bright compared to other ionized gas tracers, however, it suffers from extinction (see $§ 1.4 .2$ ). Since extinction is largest toward the inner Galaxy, $\mathrm{H} \alpha$ observations are typically restricted to outer-Galaxy sources or sources off the Galactic plane.

\subsubsection{Infrared Emission}

There is a multitude of emission lines in the IR, including recombination lines, atomic fine-structure transitions, molecular vibrational and rotational lines, and thermal continuum radiation from dust. The focus of this section lies on two specific IR bands that are strongly associated with emission from $\mathrm{H}$ II regions and PDRs: 
$\sim 24 \mu \mathrm{m}$ emission from hot, small dust grains and $\sim 8 \mu \mathrm{m}$ emission from polycyclic aromatic hydrocarbons (PAHs).

Although dust only makes up $\sim 1 \%$ of the total mass of the ISM, it absorbs a large number of photons emitted by stars. The dust is heated and subsequently re-emits in the IR, providing a significant fraction $(\sim 30 \%)$ of the total luminosity of the Milky Way (Mathis, 1990). Dust grains largely consist of silicates and carbides, and range in size from a few molecules to several $\mu \mathrm{m}$. In environments with strong radiation fields, such as H II regions, small dust grains are heated stochastically. These small grains are responsible for the strong $\sim 24 \mu \mathrm{m}$ emission observed toward H II regions.

H II region PDRs emit strongly in the mid-IR due to PAHs. PAHs are hydrocarbons composed of multiple aromatic rings, which emit by various bending and stretching modes. PAHs are excited by near-UV photons, however, very hard radiation fields are hostile to them since their dissociation energy can be as low as $3.5 \mathrm{eV}$ (Voit, 1992). The strongest PAH emission features associated with PDRs are at $\sim 8 \mu \mathrm{m}$ and $\sim 12 \mu \mathrm{m}$.

\subsection{Telescopes and Datasets Used}

\subsubsection{Green Bank Telescope}

The majority of the data used in this work comes from the Green Bank Telescope (GBT) located in Green Bank, WV. With a total collecting area of 9,300 $\mathrm{m}^{2}$, the GBT is the world's largest fully steerable single-dish radio telescope. The GBT is located at a latitude of $38.4^{\circ}$ North, resulting in a sky coverage of $\sim 85 \%$, which includes the entire first and second quadrant of the Galaxy, as well as parts of the third and fourth quadrant.

The main advantages of the GBT in observing H II regions and the diffuse ionized gas are its large collecting area, resulting in a high sensitivity (see Eq. 1.10), and its capability to simultaneously measure a large number of RRLs. While radio interferometers such as the Atacama Large Millimeter Array (ALMA) or the Jansky 
Very Large Array (VLA) have similar total collecting areas, interferometers are not sensitive to diffuse extended sources such as the WIM. The beam size of the GBT at C-band ( $4-8 \mathrm{GHz})$ is $\sim 2^{\prime}$, which is small enough to resolve most classical $\mathrm{H}$ II regions in the Milky Way, but large enough to map large areas of the sky in a reasonable amount of time. Depending on the receiver and the observing mode, the GBT can record up to 64 frequency bands simultaneously, including up to $22 \mathrm{H} n \alpha$ transitions. The Hno lines can then be averaged together to make one sensitive spectrum (e.g., Balser, 2006).

Summarized below are the GBT observations carried out for this work. In $\S 2$ we observed the compact Galactic H II region NGC 7538 in X-band radio continuum and RRL emission. We mapped the radio continuum emission from a $40^{\prime}$ square region centered at $(\ell, b)=\left(111.544^{\circ}, 0.813^{\circ}\right)$ using the Digital Continuum Receiver on the GBT. The observations were done at $8665 \mathrm{MHz}$ with a bandwidth of $320 \mathrm{MHz}$ in two orthogonal polarizations (left and right circular). The telescope was slewed at a rate of $60^{\prime} \mathrm{min}^{-1}$ and we took data by scanning in Galactic longitude and Galactic latitude to minimize in-scan artifacts. We also employed total-power position-switching RRL observations of 16 individual positions near NGC 7538. Integration times ranged from 12 to 216 minutes per position. For integration times exceeding 6 minutes, the observation was split up into blocks of 6 minutes each to reduce the overall impact of radio-frequency interference (RFI). We simultaneously measured $7 \mathrm{Hn} \alpha$ RRL transitions in two orthogonal polarizations. Each spectrum spans $50 \mathrm{MHz}$ and is sampled by 4096 channels, for a spectral resolution of $\sim 0.4 \mathrm{~km} \mathrm{~s}^{-1}$ per channel.

In $\S 3$ we use previously published (Anderson et al., 2011; Anderson et al., 2015b) and unpublished GBT RRL data of 254 discrete pointings between $\ell=18^{\circ}$ and $40^{\circ}$ and $|b|<1^{\circ}$. The pointings include directions coincident with $\mathrm{H}$ II regions, for which the diffuse gas velocity can be distinguished from the H II region velocity, as well as directions devoid of discrete HII regions. The observational setup is identical with the RRL observations in $\S 2$ described above.

In $\S 4$ we took C-band ( $4-8 \mathrm{GHz}) \mathrm{RRL}$ data toward eight Galactic H II regions with the GBT. For each targeted H II region between 7 and 17 positions were observed, 
with integration times ranging from 3 to 36 minutes per position. As in in $\S 2$, we observed in total-power position-switching mode and split up the observations into blocks of 6 minutes each for integration times exceeding 6 minutes to reduce the impact of RFI. We simultaneously observed $22 \mathrm{H} n \alpha$ transitions ( $n=95$ to $n=117$ ) in two orthogonal polarizations. We also tuned to $19 \mathrm{Hn} \beta$ lines, $8 \mathrm{Hn} \gamma$ lines, $7 \mathrm{HeIn} \alpha$ lines, and 8 molecular lines, including formaldehyde and methanol.

\subsubsection{Wide-field Infrared Survey Explorer}

WISE is a $40 \mathrm{~cm}$ IR space telescope, which produced an all-sky astronomical survey at wavelength bands of 3.4, 4.6, 12, and $22 \mu \mathrm{m}$ (Wright et al., 2010). Because H II regions have a characteristic IR morphology $-24 \mu \mathrm{m}$ emission from small dust grains (associated with the ionized gas) surrounded by 8 and $12 \mu \mathrm{m}$ emission from PAHs (associated with the PDR)-WISE survey data can be used to identify HII region candidates (see Figure 1.1). Based on this criterion, Anderson et al. (2014) compiled an H II region catalog, the "WISE Catalog of Galactic H II Regions."

Here, we use $12 \mu \mathrm{m}$ WISE data to trace H II region PDRs (see $\S 2$ and $\S 4$ ). We also use the WISE catalog to identify discrete H II regions and distinguish between emission from H II regions and WIM emission (see §3).

\subsubsection{Supplementary Data}

There are various supplementary datasets that we use for our analysis in this work. In $\S 2$ we derive dust temperatures from Herschel Space Observatory data at 160, 250, 350, and $500 \mu \mathrm{m}$ (Motte et al., 2010), and we compare H $\alpha$ data from the "Isaac Newton Telescope Photometric H-Alpha Survey of the Northern Galactic Plane" (Drew et al., 2005; Barentsen et al., 2014) with our radio continuum map. We also use $8 \mu \mathrm{m}$ emission data from the Spitzer Space Telescope (Benjamin et al., 2003; Churchwell et al., 2009) to trace PAH emission, neutral hydrogen data from the VLA Galactic Plane Survey (Stil et al., 2006) to investigate the location of the WIM, and carbon monoxide emission data from the "Milky Way in Molecular Clouds" survey 
(Dame et al., 2001) and the Galactic Ring Survey (Jackson et al., 2006, all in §3) to trace the cold molecular gas.

\subsection{Key Questions}

The main goal of this work is to better understand the connection between H II regions and the Warm Ionized Medium (WIM). Key questions we aim to answer are:

1. How is radiation originating from the O-stars within Galactic H II regions able to leak into the ISM?

2. How does the radiation field change as the photons escape through the HII region $\mathrm{PDR}$ ?

3. What fraction of ionizing radiation leaks from individual H II regions and how does this radiation affect the ambient ISM?

4. What are the large-scale spatial distribution, kinematics, and physical properties of the WIM?

\subsection{Dissertation Outline}

The following chapters each analyze a different aspect of the connection between H II regions and the WIM.

In $\S 2$ we focus our analysis on the compact H II region NGC 7538. We quantify the leaking fraction of NGC 7538 and constrain the hardness of the radiation field. By comparing our data of NGC 7538 with the H II region complex W43, we explore if high-luminosity H II regions have a stronger effect on maintaining the ionization of the WIM compared to regions with lower luminosity.

In $\S 3$ we study the distribution of the WIM in the first Galactic quadrant. We constrain the large-scale kinematics of the WIM and calculate possible distances for the strongest emission components. We also explore the correlation between hard and soft-UV radiation to better understand the origin of the interstellar radiation field. 
In $\S 4$ we analyze a larger sample of Galactic H II regions to determine whether the results derived in $\S 2$ are representative of the $\mathrm{H}$ II region population as a whole. We also calculate physical properties of the observed H II regions and quantify the impact of leaking radiation on the ambient ISM.

We discuss current and future plans in $\S 5$, and summarize in $§ 6$. 


\section{Chapter 2}

\section{H II Region Ionization of the Interstellar Medium: A Case Study of NGC 7538}

\subsection{Abstract}

Using data from the Green Bank Telescope, we analyze the radio continuum (free-free) and radio recombination line (RRL) emission of the compact H II region NGC 7538 (Sharpless 158). We detect extended radio continuum and hydrogen RRL emission beyond the photodissociation region (PDR) toward the north and east, but a sharp decrease in emission toward the south and west. This indicates that a nonuniform PDR morphology is affecting the amount of radiation "leaking" through the PDR. The strongest carbon RRL emission is found in the western PDR that appears to be dense. We compute a leaking fraction $f_{\mathrm{R}}=15 \pm 5 \%$ of the radio continuum emission measured in the plane of the sky which represents a lower limit when accounting for the three-dimensional geometry of the region. We detect an average ${ }^{4} \mathrm{He}^{+} / \mathrm{H}^{+}$ abundance ratio by number of $0.088 \pm 0.003$ inside the $\mathrm{H}$ II region and a decrease in this ratio with increasing distance from the region beyond the PDR. Using Herschel Space Observatory data, we show that small dust temperature enhancements to the north and east of NGC 7538 coincide with extended radio emission, but that the dust temperature enhancements are mostly contained within a second PDR to the east. Unlike the giant H II region W43, the radiation leaking from NGC 7538 seems to only affect the local ambient medium. This suggests that giant H II regions may have a large effect in maintaining the ionization of the interstellar medium.

The research contained in this chapter was published as Luisi et al. (2016), ApJ, 824, 125.

Contributing authors: L. D. Anderson, D. S. Balser, T. M. Bania, T. V. Wenger. 


\subsection{Introduction}

Despite the fact that they are relatively rare, O-type stars have a large impact on the interstellar medium (ISM) at both large and small spatial scales. Their ultraviolet (UV) photons propagate through molecular clouds, dissociating molecules and ionizing the gas. Due to their intense radiation fields, O stars are surrounded by H II regions of ionized plasma (see Höglund \& Mezger, 1965; Mezger et al., 1967). At the interface between the fully ionized H II region and the neutral medium surrounding it there is a photo-dissociation region (PDR). The ionized gas within H II regions can be studied using radio recombination line (RRL) and radio free-free continuum emission, which have the benefit of being essentially free from the effects of extinction, whereas their PDRs can be studied using numerous molecular or atomic transitions.

The ISM of galaxies like the Milky Way contains low-density $\left(\sim 0.1 \mathrm{~cm}^{-3}\right)$ diffuse ionized gas known as the "warm interstellar medium" (WIM), first proposed by Hoyle \& Ellis (1963). The WIM is a major, widespread component of the ISM, with a scale height of $\sim 1500 \mathrm{pc}$ and temperatures between 6,000 and 10,000 K (Reynolds, 1989; Haffner et al., 2009). Optical emission line measurements have shown that the WIM is in a lower ionization state and is ionized by a softer radiation field compared to gas in H II regions (Madsen et al., 2006). The "extended low-density medium" (ELDM; see Gottesman \& Gordon, 1970; Mezger, 1978) is occasionally cited as another diffuse ionized component of the ISM, with a smaller scale height of $\sim 100 \mathrm{pc}$ and a density

of 1 to $10 \mathrm{~cm}^{-3}$. The distribution of the ELDM was found to be correlated with the location of H II regions (Alves et al., 2012).

It is still not completely understood how the WIM maintains its ionization (Haffner et al., 2009). While the radiation from supernovae can contribute, it cannot provide the total energy required (Hoopes \& Walterbos, 2003). The most likely source of ionizing photons is O-type stars (Domgörgen \& Mathis, 1994; Madsen et al., 2006), but it is unclear precisely how radiation from the luminous O-type stars is able to propagate across the kiloparsec size-scales required given the distribution of the WIM. One suggestion is that superbubbles created by supernovae and stellar winds provide 
low-density regions for photons to traverse Galactic distances (see Cox \& Smith, 1974; Dove et al., 2000; Reynolds et al., 2001; Terebey et al., 2003; Dale et al., 2005). Another possibility is the existence of a two-component (Wood \& Loeb, 2000) or fractal (Ciardi et al., 2002) ISM with a sufficient number of low-density paths.

If the O-type stars within H II regions are maintaining the ionization of the WIM, their radiation must either escape through their dense PDRs or their PDRs must be clumpy. In a clumpy PDR, photons could escape along low-density pathways in some directions and heat the ambient dust. For the Galactic H II region RCW 120, Anderson et al. (2010) found dust temperature enhancements that correlate with locations where the PDR shows discontinuities at $8.0 \mu \mathrm{m}$, which suggests that for this region radiation is leaking through such small-scale $(\sim 0.3 \mathrm{pc})$ inhomogeneities in the PDR. This result was supported by CO observations by Anderson et al. (2015), who determined that these holes are spatially correlated with deficits in CO emission at distinct molecular velocities. They further noted that $\sim 5 \%$ of the $\mathrm{H} \alpha$ emission of RCW 120 (as measured in the plane of the sky) is found directly outside these holes and that overall RCW 120 is leaking $\sim 25 \pm 10 \%$ of its emission beyond its PDR. Extended radio continuum and RRL emission has also been observed by Kim \& Koo (2001) around ultracompact H II regions. They argue that these extended emission envelopes are mostly due to ionizing radiation from the exciting star of the compact region.

In a survey of $117 \mathrm{H}$ II regions with multiple hydrogen RRL velocities, Anderson et al. (2015b) found that most multiple-velocity regions are clustered near large starforming complexes in the inner Galaxy. They posited that the additional velocity components are caused by diffuse ionized gas along the line of sight, perhaps due to photons from these large complexes leaking into the ISM. Observations in Anderson et al. (2011) support this hypothesis, as they found that for multiple-velocity HII regions near W43, the strength of the diffuse velocity component decreases with increasing projected distance from the central position of W43. This suggests that leaking photons from W43 may be a major contributor to the WIM in these directions.

The main goal of the present study is to understand how a single H II region 
may contribute to the diffuse ionized gas detected by Anderson et al. (2011). While the Anderson et al. (2015b) study suggests a link between H II regions and diffuse ionized gas, additional research on individual regions is required. The region W43 is at the tip of the Galactic bar, along a very complicated sight line, so the relationship between the diffuse ionized gas and H II regions is still not clear.

Numerous studies of external galaxies indicate that a significant amount of ionizing radiation may leak from H II regions. Averaged over the entire NGC 157 galaxy, Zurita et al. (2002) found that $30 \%$ of the emitted ionizing photons escape from H II regions based on their $\mathrm{H} \alpha$ emission model. Though this escape fraction by itself may not be sufficiently large to account for the total luminosity of the diffuse ionized gas, they suggested that almost all ionizing radiation escapes from the highest luminosity H II regions. As a result, the ionization of the diffuse gas could be maintained even with a much lower escape fraction of less luminous regions (see Zurita et al., 2000). Oey \& Kennicutt (1997) compared the estimated stellar Lyman continuum flux to the $\mathrm{H} \alpha$ luminosities of the H II regions for the Large Magellanic Cloud. They estimated that up to $50 \%$ of ionizing radiation escapes the nebulae. Pellegrini et al. (2012) used photoionization models of optically thin H II regions. They calculated a lower limit on the ionizing escape fraction of $42 \%$ for the Large Magellanic Cloud and $40 \%$ for the Small Magellanic Cloud. Giammanco et al. (2005) showed that models with optically thick clumps within the H II region are generally in better agreement with observations than models that allow photon transmission through optically thin clumps. They further found photon escape fractions of 30\%-50\% for M51 and $<60 \%$ for M101.

The spectrum of the stellar radiation field outside an $\mathrm{H}$ II region PDR differs from that inside as some of the leaked photons undergo absorption and re-emission processes in the local ISM. Though these processes can be complex, a hardening in the H-ionizing continuum and a suppression of He-ionizing photons has been observed (Wood \& Mathis, 2004). As there will be fewer photons with enough energy to ionize $\mathrm{He}$ compared to $\mathrm{H}$ outside the $\mathrm{H}$ II region, the observed ionic abundance ratio $N\left({ }^{4} \mathrm{He}^{+}\right) / N\left(\mathrm{H}^{+}\right)$should thus be lower than it is inside (see Roshi et al., 2012). As well 
as photons being absorbed by the gas around $\mathrm{H}$ II regions, part of the radiation field is attenuated by interstellar dust. This becomes particularly important in PDRs, as they can absorb UV photons from the central source and re-emit them in the infrared (Hollenbach \& Tielens, 1997a). Knowledge of the dust properties in and around the PDR can provide further understanding of the radiative transfer effects in these regions (see Compiegne et al., 2008).

Here we use radio observations of the H II region NGC 7538 as a case study to understand the role that leaking UV radiation from H II regions may have in creating the diffuse ionized gas detected by Anderson et al. (2011), and to better understand the connection between this diffuse ionized gas in the vicinity of $\mathrm{H}$ II regions and the WIM. NGC 7538 is located in the Perseus spiral arm at a distance of $2.65 \mathrm{kpc}$ (see Moscadelli et al., 2009) and is therefore close enough for detailed study. It lies in the Outer Galaxy where there is less confusion along the line of sight. We describe the radio continuum and RRL observations in Section 2.3 of this dissertation. We derive an estimate of the location of the PDR boundary, determine the percentage of leaking emission, and analyze the properties of ionized gas outside the PDR in Section 2.4. We discuss these results in Section 2.5 and conclude in Section 2.6.

\subsection{Observations}

We observed NGC 7538 in radio continuum and RRL emission at X-band (9 GHz; $3 \mathrm{~cm}$ ) using the National Radio Astronomy Observatory Green Bank Telescope (NRAO GBT) from February 2013 to March 2014. For both the continuum and line data, we assume that the noise diodes, fired during data acquisition, provide accurate intensities at the $10 \%$ level. We verified the flux density calibration of $3 \mathrm{C} 147$ in a nearly concurrent program in November 2012 that used the same instrumental configuration (Anderson et al., 2015a) and found agreement with Peng et al. (2000) to within $10 \%$. Throughout, we use a GBT X-band gain of $2 \mathrm{~K} \mathrm{Jy}^{-1}$ to convert from antenna temperature to flux density (Ghigo et al., 2001). 


\subsubsection{Radio Continuum}

We mapped the radio continuum emission from a $40^{\prime}$ square region centered at $(\ell, b)=\left(111.544^{\circ}, 0.813^{\circ}\right)$ or $(J 2000$ R.A., decl. $)=(23: 13: 40,61: 30: 13)$ using the Digital Continuum Receiver (DCR) on the GBT. Our central position for NGC 7538 corresponds to the $12 \mu \mathrm{m}$ Wide-Field Infrared Survey Explorer catalog (WISE; see Wright et al., 2010). The observations were done at $8665 \mathrm{MHz}$ with a bandwidth of $320 \mathrm{MHz}$ in two orthogonal polarizations (left and right circular). Radio continuum emission from H II regions at this frequency is caused by free-free emission. We slewed the telescope at a rate of $60^{\prime} \mathrm{min}^{-1}$, while sampling the total power every $100 \mathrm{~ms}$. We took data by scanning in Galactic longitude and Galactic latitude, and created maps by averaging the two directions and polarizations to minimize in-scan artifacts.

We show the radio continuum image in Figure 2.1. Here, all references to east or west refer to increasing or decreasing Galactic Longitude, respectively, and references to north or south refer to increasing or decreasing Galactic Latitude. Most of the emission is peaked slightly west of the central location, but there is extended emission toward the east that appears in two lobes. There is also faint extended emission toward the north, but little toward the south and west.

\subsubsection{Radio Recombination Lines}

We made RRL observations using the AutoCorrelation Spectrometer (ACS) on the GBT using our standard techniques (Bania et al., 2010; Balser et al., 2011; Anderson et al., 2011). We employed position switching with On- and Off-source integrations of 6 minutes per scan. The Off-source scans tracked the same azimuth and zenith angle path as the On-source scans and were observed offset 6.5 minutes in right ascension. We simultaneously measured $7 \mathrm{Hn} \alpha$ RRL transitions, H87 $\alpha$ to H93 $\alpha$ (at rest frequencies of 9812.0, 9505.0, 9183.0, 8877.0, 8584.8, 8300.0, and 8045.6 MHz), in two orthogonal polarizations. Each observation therefore resulted in 14 independent spectra. Each spectrum spans $50 \mathrm{MHz}$ and is sampled by 4096 channels, for a spectral resolution of $\sim 0.4 \mathrm{~km} \mathrm{~s}^{-1}$ per channel. The GBT half-power beamwidth (HPBW) at 

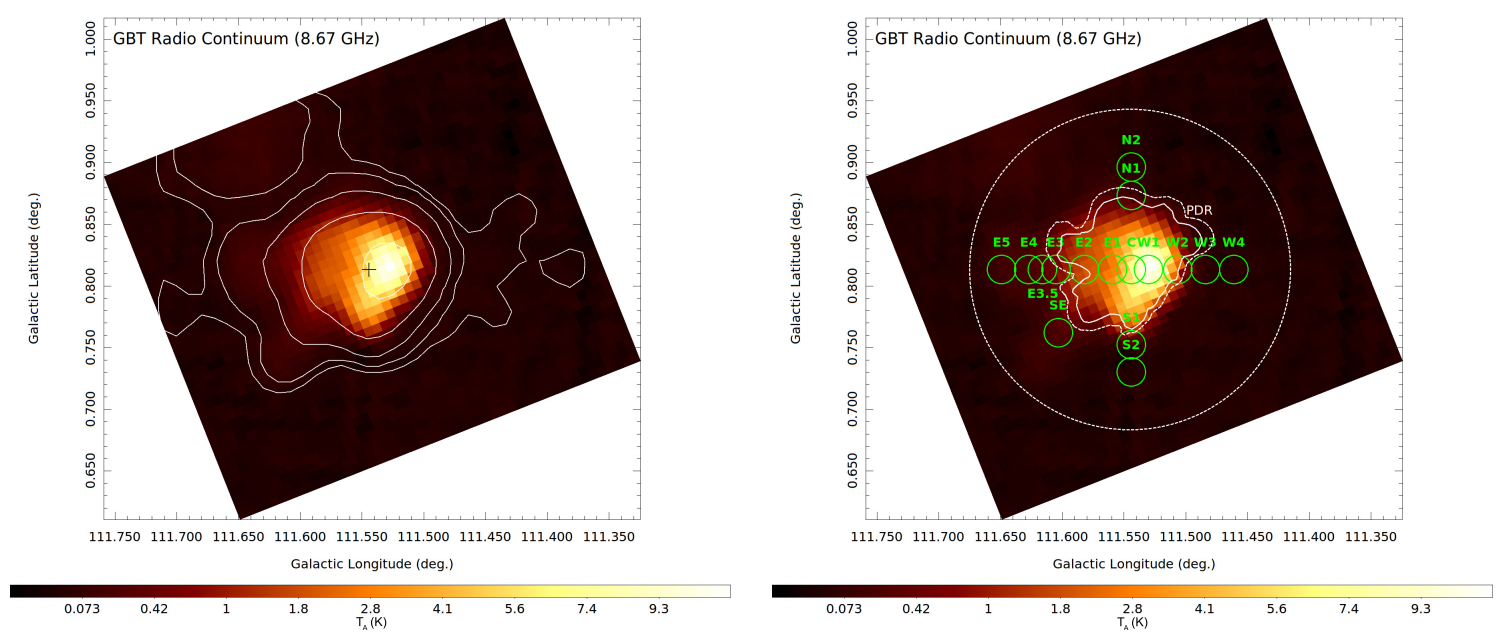

Figure 2.1: Left: Radio continuum image of NGC 7538 at $8.67 \mathrm{GHz}$ in units of antenna temperature. The contours start at $T_{\mathrm{A}}=0.01 \mathrm{~K}$ and increase logarithmically to a maximum of $5 \mathrm{~K}$. The black cross marks the central position (position "C" in the right image) of the region. Right: Same image as left. The solid and dashed white regions marked "PDR" represent the inner and outer PDR boundary of NGC 7538 defined in Figure 2.3 (see text). Green circles indicate the positions where RRLs were observed. The size of the circles is that of the GBT beam at $8.67 \mathrm{GHz}\left(82^{\prime \prime}\right)$. Positions are labeled as in Table 2.1. We use the area inside the large white dashed circle to derive the total intensity of the region. 
these frequencies ranges from $73^{\prime \prime}$ to $90^{\prime \prime}$. We take the average of $82^{\prime \prime}$ for all further RRL data analyses.

Figure 2.1 shows our GBT $8.7 \mathrm{GHz}$ radio continuum map of NGC 7538 overlaid with the positions of the RRL measurements. We observed a total of 16 positions, of which 15 are in a "cross" with arms of constant latitude and longitude to trace variations across the PDR boundaries. Eleven of the observed positions are on a line of constant Galactic Latitude, which we refer to as the East-West (EW) direction, and five positions are on a line of constant Galactic Longitude. The cross directions intersect at the central position at $(\ell, b)=\left(111.544^{\circ}, 0.813^{\circ}\right)$ or $(\mathrm{J} 2000$ R.A., decl. $)=$ (23:13:39.8, 61:30:13). We observed one additional position offset to the south-east at $(\ell, b)=\left(111.603^{\circ}, 0.762^{\circ}\right)$ or $(\mathrm{J} 2000$ R.A., decl. $)=(23: 14: 16.5,61: 28: 40)$. Based on our definition of the location of the PDR boundary of NGC 7538 (see Section 2.4.1), most of the observed positions are outside the PDR, with only three positions located within the PDR and two on the PDR.

We reduce and analyze the data in TMBIDL ${ }^{1}$ (Bania et al., 2014). For each position we average all 14 spectra together to increase the RRL signal-to-noise ratio (see Balser, 2006) after first re-gridding to the velocity resolution of the $\mathrm{H} 87 \alpha$ data (which has the poorest resolution) and shifting the spectra so they are aligned in velocity. When averaging, we use a weighting factor of $t_{\text {intg }} / T_{\text {sys }}^{2}$, where $t_{\text {intg }}$ is the integration time and $T_{\text {sys }}$ is the system temperature. We smooth the resultant spectrum to a resolution of $1.86 \mathrm{~km} \mathrm{~s}^{-1}$ and fit a third-order polynomial baseline. We fit Gaussian models to the $\mathrm{H}, \mathrm{He}$, and $\mathrm{C}$ profiles if their peak signal was two times the rms noise defined in a line-free portion of the spectrum. We thus derive the peak line heights, their full width at half maximum (FWHM) values, and LSR velocities for all detected $\mathrm{H}, \mathrm{He}$, and $\mathrm{C}$ components. In spectra with strong He lines, the carbon RRLs are often blended with He. This makes it difficult to fit both lines simultaneously. In these cases, we fit the He RRLs first, subtract the He Gaussian models, and then fit the C lines (see Quireza et al., 2006). In Figure 2.2, we show the RRL data and Gaussian fits from all observed positions.

\footnotetext{
${ }^{1}$ V7.1, see https://github.com/tvwenger/tmbidl.git.
} 

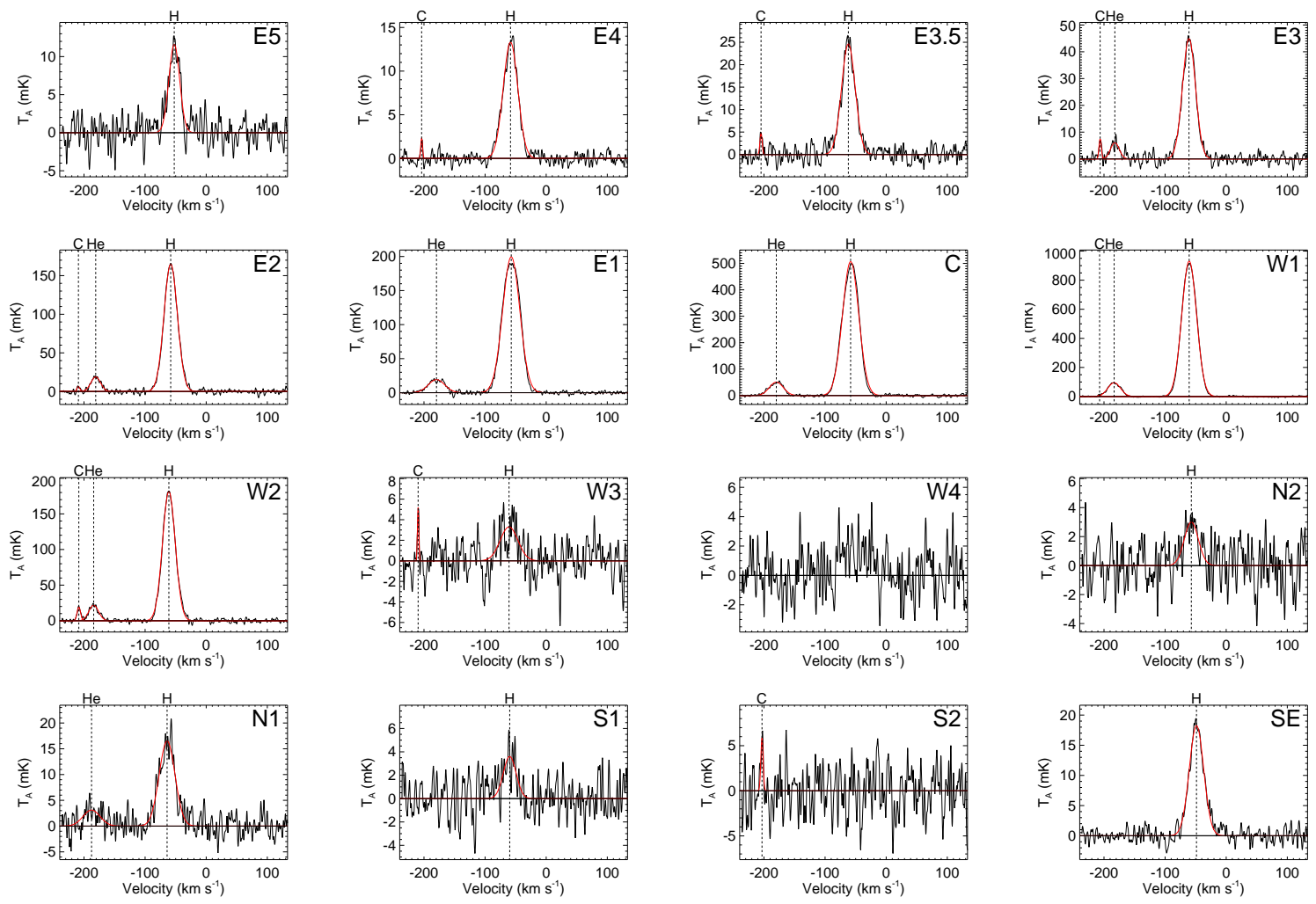

Figure 2.2: RRL spectra of the 16 observed positions in NGC 7538, smoothed to a spectral resolution of $1.86 \mathrm{~km} \mathrm{~s}^{-1}$. Plotted is the antenna temperature as a function of LSR velocity. Gaussian model fits to the $\mathrm{H}, \mathrm{He}$, and $\mathrm{C}$ peaks above the $2 \sigma$ confidence interval are shown. The centers of the Gaussian peaks are indicated by dashed vertical lines. 
Of the 16 positions observed, we detect a hydrogen line at 14 positions, a helium line at 7 positions, and a carbon line at 8 positions. We summarize the observations and RRL analysis in Table 2.1, which lists the source, the Galactic longitude and latitude, the location of the observed position with respect to the PDR based on our PDR definition in Section 2.4.1 ("on" for on the PDR, "in" for inside the PDR, "out" for outside the PDR), the line, the line intensity, the FWHM line width, the LSR velocity, the rms noise in the spectrum, and the total integration time for each position, including all corresponding $1 \sigma$ uncertainties of the Gaussian fits.

\subsection{Ionized Gas in and around NGC 7538}

\subsubsection{The PDR Boundary}

If the origin of the WIM is linked to escaping ionizing photons from H II regions, we should detect a significant amount of radio continuum emission "leaking" through the PDR. In order to quantify this amount, we need to define the inner PDR boundary. Radio emission outside this boundary can then be classified as "leaked", whereas emission inside the boundary can be associated with the H II region itself.

We use the $12 \mu \mathrm{m}$ WISE map shown in Figure 2.3 to define inner and outer PDR boundaries. Within the $12 \mu \mathrm{m}$ band is strong emission from a polycyclic aromatic hydrocarbon (PAH; e.g. Hollenbach \& Tielens, 1997b). This emission occurs in PDRs of H II regions, allowing us to use the image to estimate the PDR extent. Following the enhanced $12 \mu \mathrm{m}$ intensity, we trace by hand the inner and outer PDR boundaries around the region. While the PDR to the north and east of the region is bright and well-defined at $12 \mu \mathrm{m}$, the sharp decrease in intensity to the south-west makes a clear distinction from the surrounding medium more difficult. 


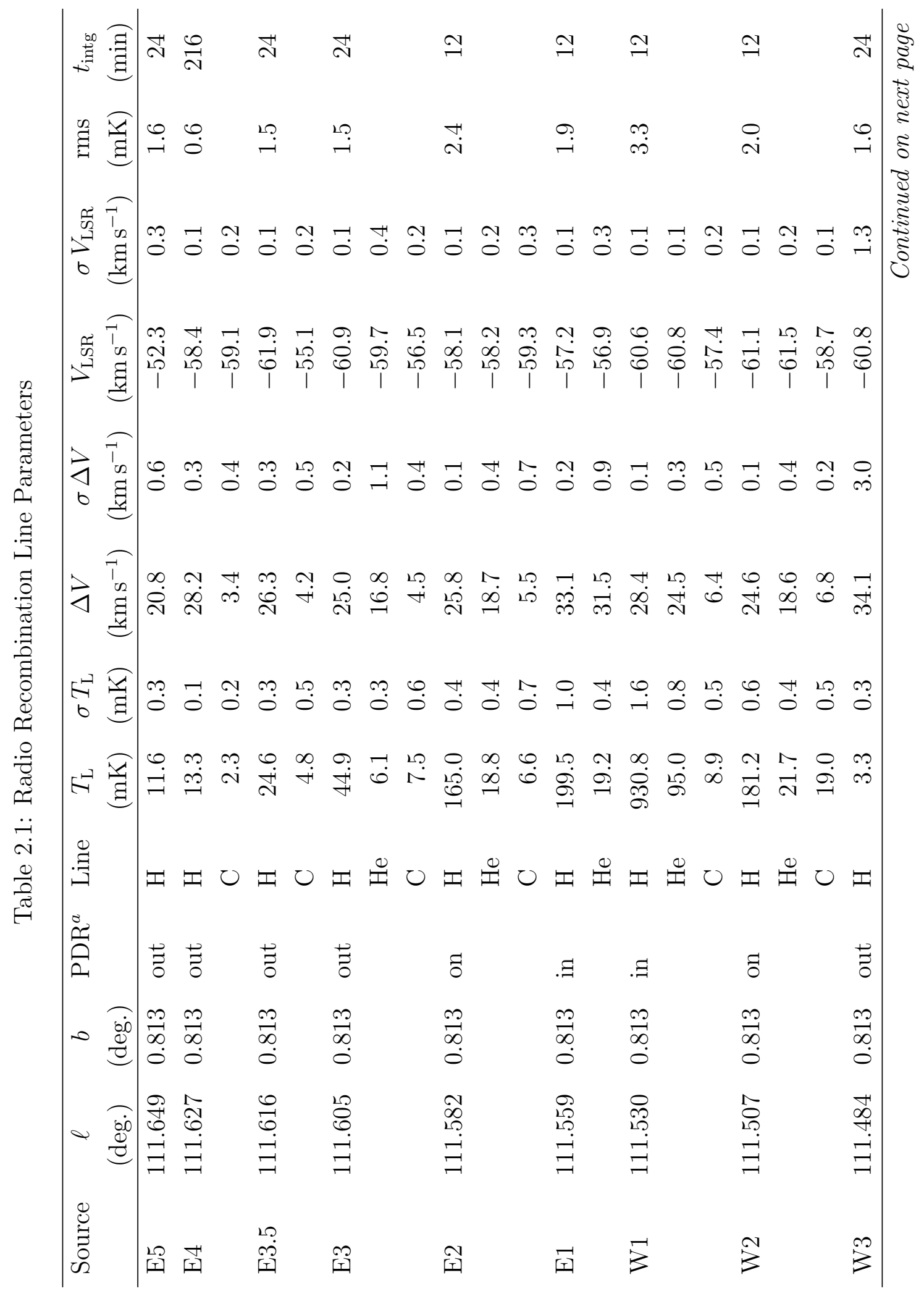




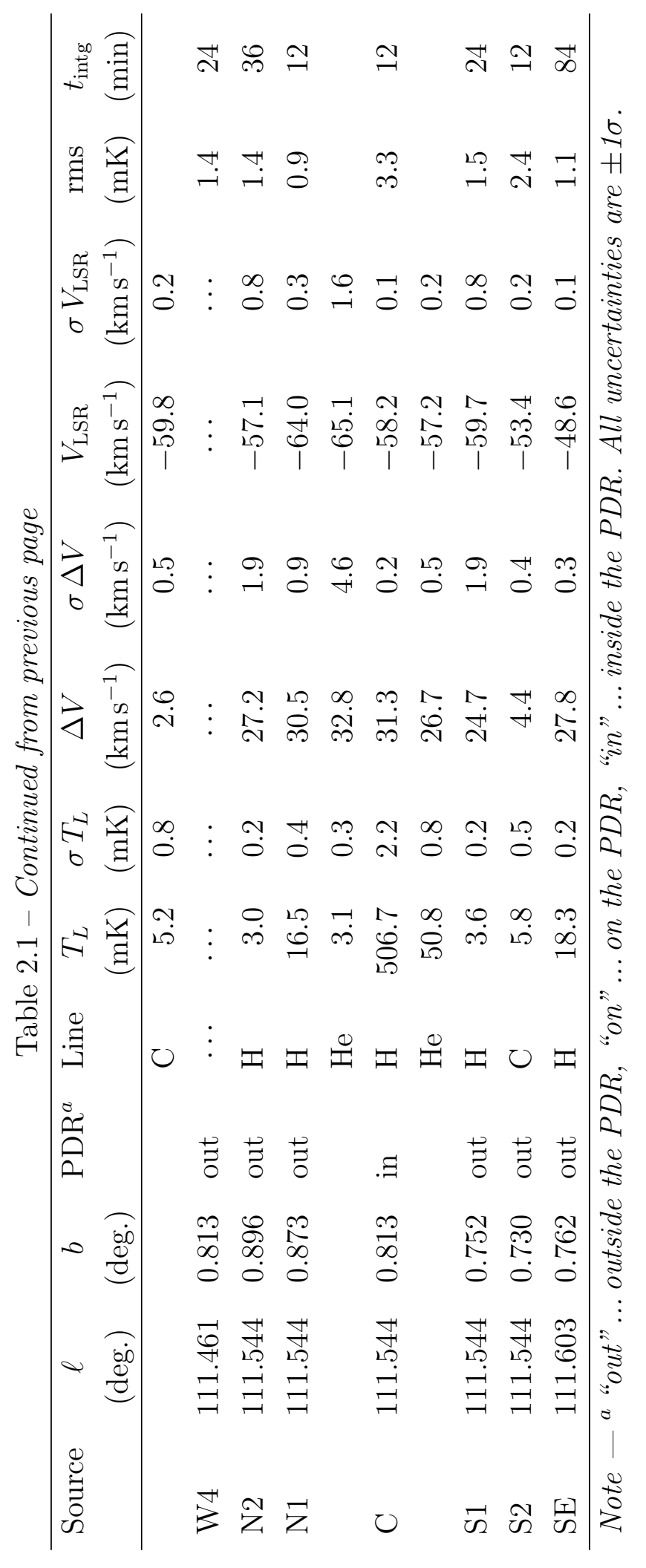


To test the plausibility of this PDR definition along the EW direction (the line of $b=0.813^{\circ}$ ), we convolve the WISE $12 \mu \mathrm{m}$ data with a Gaussian of FWHM 87", the HPBW of the GBT at the frequency of the radio continuum data (Figure 2.4). This gives us a smoothed intensity distribution of the $12 \mu \mathrm{m}$ emission along the EW direction that can easily be compared to the radio data. We assume that the center of the PDR can be identified from peaks in the WISE intensity, which are located at $(\ell, b)=\left(111.585^{\circ}, 0.813^{\circ}\right)$ and $(\ell, b)=\left(111.503^{\circ}, 0.813^{\circ}\right)$, respectively. We then fit a Gaussian to these peaks and define the width of the PDR as the FWHM of the fits, resulting in a total width of $0.012^{\circ}$ for the eastern PDR boundary and $0.010^{\circ}$ in the west. Due to the convolution with the GBT HPBW these widths may be broadened. This analysis agrees well with the visual definition (see Figure 2.4). PDRs, however, are characterized by a multitude of emission lines (Hollenbach \& Tielens, 1997b). Using other emission lines to trace the PDR may shift its location and therefore our characterization of the PDR structure is by no means unique. Throughout the remainder of this work we use the visual definition of the PDR boundary defined in Figure 2.3.

There is, however, a second PDR structure visible to the east in the WISE $12 \mu \mathrm{m}$ data of Figure 2.3 (indicated by the dashed white curve). This second PDR may be an additional boundary that prevents photons from escaping great distances from the region. This second PDR is barely visible as a slight enhancement in Figure 2.4 at $\ell=+0.12$.

\subsubsection{The Leaking Emission Fraction}

Since extinction due to interstellar dust is negligible at $\nu \approx 8.7 \mathrm{GHz}$, we can use the radio continuum intensity to estimate the percentage of emission escaping the inner PDR boundary of the region. To determine this percentage of leaked emission, or escape fraction $f_{\mathrm{R}}$, from the radio data the intensity inside and outside the PDR boundary must be known. Assuming that the continuum emission is completely thermal and that the continuum background intensity is negligible, we sum the radio continuum map pixel values inside the visually-defined PDR boundary to find the 


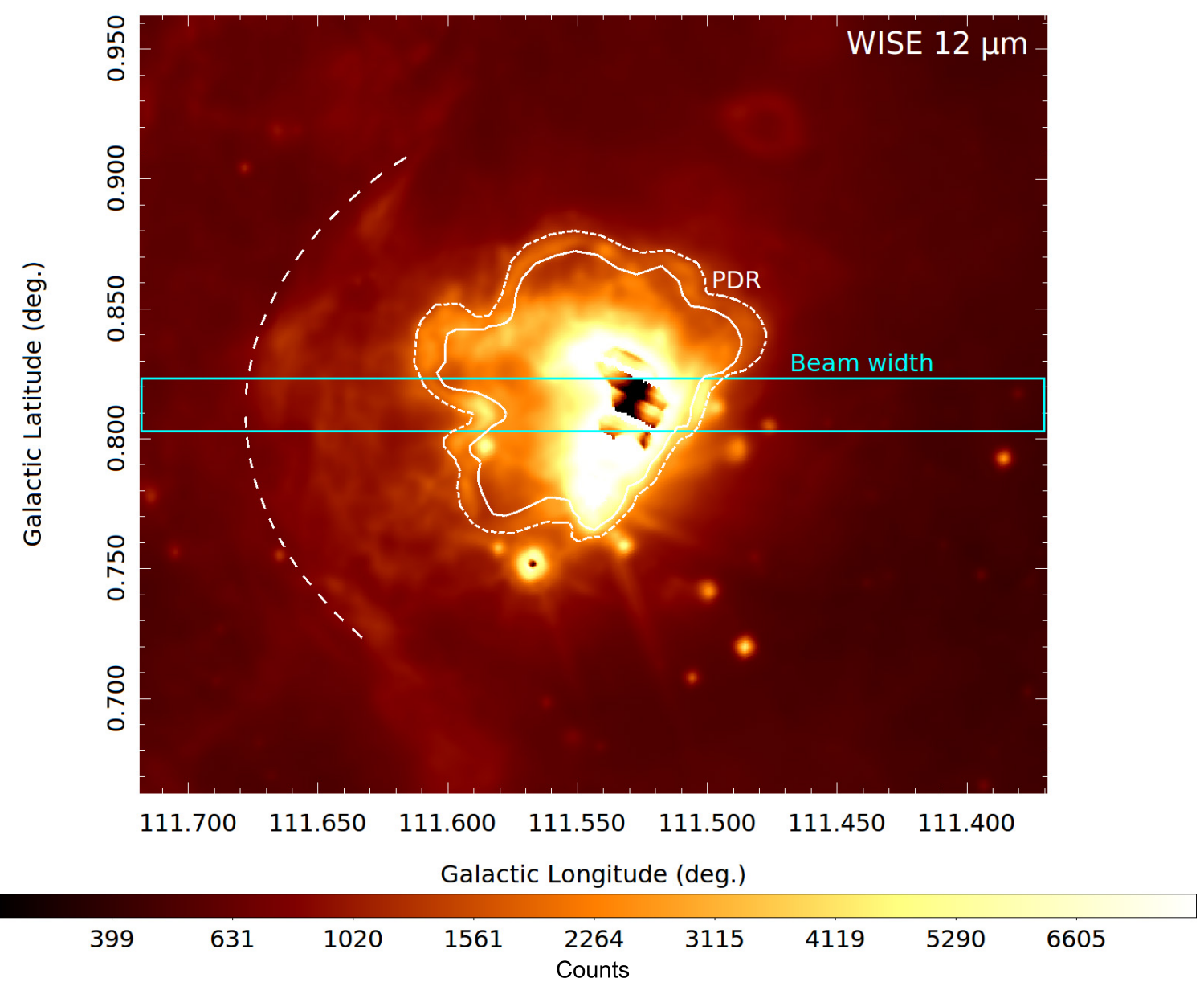

Figure 2.3: WISE $12 \mu \mathrm{m}$ image showing PAH emission from the region. The profile shown in Figure 2.4 is extracted from the blue box area. The width of the box is the GBT beam at $8.67 \mathrm{GHz}$ and the vertical center of the box is at $b=0.813^{\circ}$. Our definitions of the inner and outer PDR boundaries are indicated by solid and dashed white lines marked "PDR", respectively. Another PDR boundary to the east is shown with the dashed white line. Portions of the interior of NGC 7538 are saturated in these data; we disregard these regions in the analysis. 


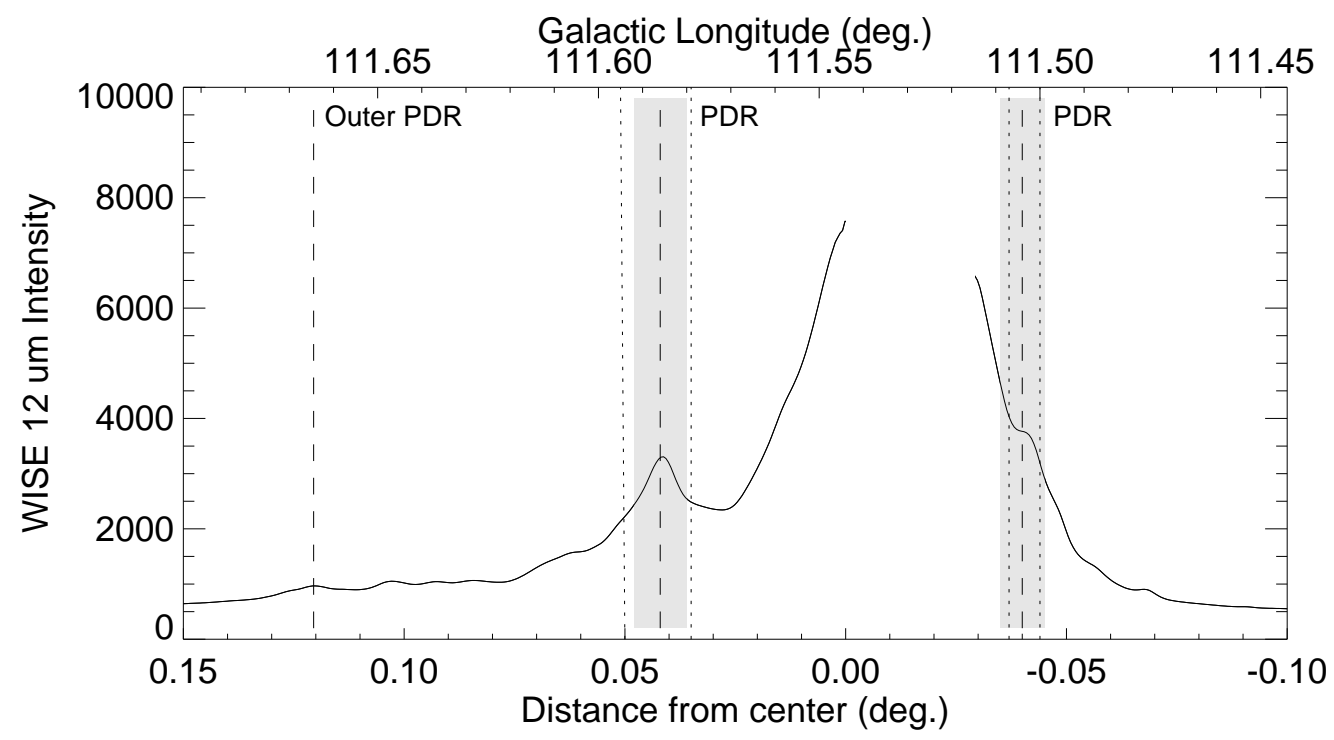

Figure 2.4: Average of the WISE $12 \mu \mathrm{m}$ intensity convolved with the GBT beam for the boxed zone shown in Figure 2.3. Missing data toward the center of the region were discarded due to saturation. We assume the center of the PDR is at the relative peaks in intensity at positions $(\ell, b)=\left(111.586^{\circ}, 0.813^{\circ}\right)$ and $(\ell, b)=\left(111.504^{\circ}, 0.813^{\circ}\right)$ as represented by the vertical dashed lines. We estimate the FWHM width of the PDR using Gaussian fits to these data, as indicated by the shaded regions. The vertical dotted lines indicate the inner and outer PDR boundaries defined visually in Figure 2.3. The second, outer PDR to the east is shown as the dashed vertical line at $\sim 0.12^{\circ}$. 
radio continuum intensity of the $\mathrm{H}$ II region. We then estimate the total intensity (inside and outside the PDR boundary) by summing the pixel values from a circle of radius $0.13^{\circ}$ centered at $(\ell, b)=\left(111.545^{\circ}, 0.813^{\circ}\right)$ as shown in Figure 2.1. A faint source of radio continuum emission is found to the north-eastern side of NGC 7538 . We assume it is from a background radio source and manually subtract its contribution to the intensity by excluding the source from the outside aperture. Since the source is faint and the continuum background intensity is insignificant, we assume that the uncertainty associated with the source subtraction is negligible.

Using a gain of $2 \mathrm{~K} \mathrm{Jy}^{-1}$, we derive the total flux densities inside and outside the PDR boundary of 17.3 Jy and 3.0 Jy, respectively, for 20.3 Jy total. Our flux density value for NGC 7538 itself (inside the PDR) is in rough agreement with those found by other authors. Gregory \& Condon (1991) found a value of $15.5 \mathrm{Jy}$ at $4.85 \mathrm{GHz}$ and Becker et al. (1991) found 23.7 Jy at 4.85 GHz.

We can calculate the expected flux density by making assumptions about the ionizing sources in NGC 7538. If two stars, O3V and O9V, are responsible for the observed emission (Puga et al., 2010), we estimate the number of emitted H-ionizing photons per second to be $N_{\text {ly }}=10^{49.65}$ (see Martins et al., 2005). From this, we calculate the expected flux density using (see Rubin, 1968; Anderson, 2010)

$$
N_{\mathrm{ly}}=4.76 \cdot 10^{48}\left(\frac{S_{\nu}}{\mathrm{Jy}}\right)\left(\frac{T_{\mathrm{e}}}{\mathrm{K}}\right)^{-0.45}\left(\frac{\nu}{\mathrm{GHz}}\right)^{0.1}\left(\frac{d}{\mathrm{kpc}}\right)^{2},
$$

where $N_{\text {ly }}$ is the number of $\mathrm{H}$-ionizing photons per second, $S_{\nu}$ is the integrated flux density at frequency $\nu, T_{\mathrm{e}}$ is the electron temperature of the region (see Section 2.4.5), and $d$ is the distance to NGC 7538. This yields an expected flux density of $60 \mathrm{Jy}$, about three times the measured value. There is, however, debate about the spectral classification of the ionizing sources in NGC 7538. Puga et al. (2010) identify the main source of H-ionizing photons as a O3V star, whereas Ojha et al. (2004) classify it as a O5V star. Following the classification of Ojha et al. (2004), we would get an estimated $S_{\nu}$ of $22.4 \mathrm{Jy}$, much closer to the measured value of 20.3 Jy. Moreover, part of the radiation field may be absorbed by dust grains within the ionized region (see 
Arthur et al., 2004) which would lower the observed flux density.

We calculate the escape fraction, $f_{\mathrm{R}}=15 \pm 5 \%$, from the ratio of the outside to total (inside plus outside) radio continuum flux density using the inner PDR boundary from Figure 2.1. Using the outer PDR as our boundary, $f_{\mathrm{R}}$ decreases to $\sim 8 \%$. We estimate the uncertainty in $f_{\mathrm{R}}$ from two contributions. We measure the effect of the uncertainty in the PDR location by shifting the PDR by half its thickness and recomputing $f_{\mathrm{R}}$. This gives a variation of $\sim 30 \%$ in $f_{\mathrm{R}}$, which is the largest contribution to the total uncertainty. We also evaluate uncertainties in the flux measurements due to the noise in the radio continuum map ( $\sim 10 \%$ contribution in $f_{\mathrm{R}}$ ). The uncertainty in $f_{\mathrm{R}}$ takes into account both effects, added in quadrature.

As an alternative method, we measure the $656 \mathrm{~nm} \mathrm{H} \alpha$ escape fraction of NGC 7538 using the Isaac Newton Telescope Photometric H-Alpha Survey of the Northern Galactic Plane (IPHAS) shown in Figure 2.5 (Drew et al., 2005; Barentsen et al., 2014). The H $\alpha$ IPHAS map has $~ 80$ times higher spatial resolution than the radio data. The disadvantages of using $\mathrm{H} \alpha$ is that extinction affects the results to an unknown degree and stellar subtraction in regions of bright $\mathrm{H} \alpha$ emission is difficult. We determine the leaking emission fraction $f_{\mathrm{H} \alpha}$ using the same methods as for the radio continuum data (i.e., without performing stellar subtraction) and find $f_{\mathrm{H} \alpha}=$ $31 \pm 10 \%$. The uncertainty includes the same contributions used for the radio data. These, added in quadrature, result in an estimated uncertainty of $\sim 5 \%$. Since the southern region of NGC 7538 shows less emission in $\mathrm{H} \alpha$ inside the PDR compared to the radio map, we assume the $\mathrm{H} \alpha$ data is affected by extinction here, which would artificially increase $f_{\mathrm{H} \alpha}$. To account for this, we add an additional $5 \%$ uncertainty contribution estimated by measuring variations in the $\mathrm{H} \alpha$ to radio continuum intensity ratio.

Because of the rather large GBT beam compared to the size of the region, some of the radio continuum emission that appears outside the H II region PDR may actually be from inside the PDR. To investigate the magnitude of this effect, we smooth the H $\alpha$ map with the GBT's $~ 87^{\prime \prime}$ beam. We find the same leaking fraction, $f_{\mathrm{H} \alpha \text {,smooth }}=31 \pm 10 \%$, indicating that the GBT beam size has not significantly 


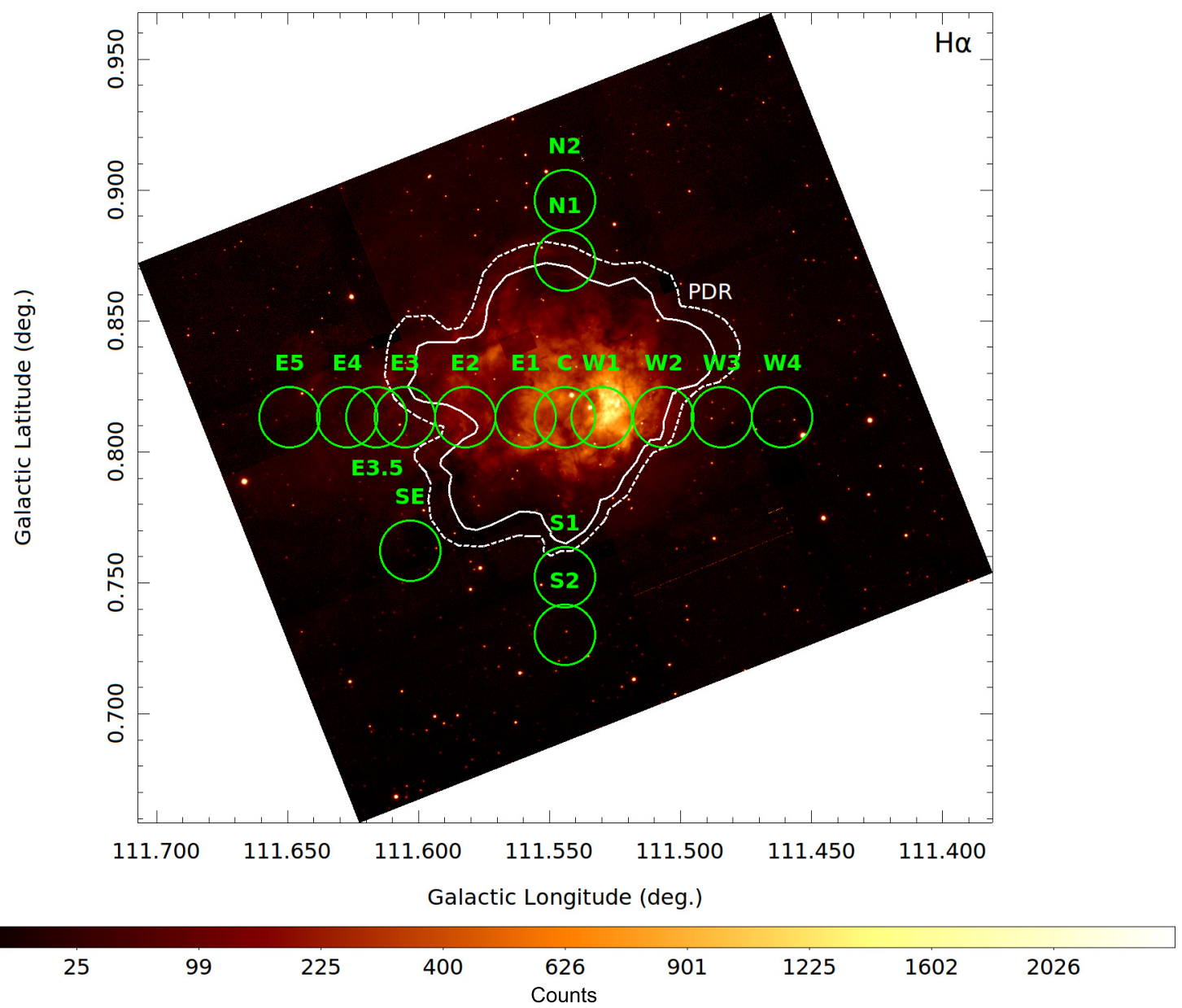

Figure 2.5: H $\alpha$ emission image of NGC 7538. The solid and dashed white regions represent the inner and outer PDR boundaries as defined in Figure 2.3. As in Figure 2.1, green circles indicate the positions where RRLs were observed. 
affected our determination of $f_{\mathrm{R}}$.

Since NGC 7538 is observed in the plane of the sky, we cannot readily distinguish emission beyond the PDR along the line of sight from that within the PDR. This effect would artificially increase the amount of emission we associate with the H II region since "leaked" emission is superposed on top of the region itself. As a result, $f_{\mathrm{R}}$ and $f_{\mathrm{H} \alpha}$ represent lower limits on the total leaking emission fraction.

\subsubsection{Ionic Abundance Ratio}

We use our RRL measurements to characterize the state of the ionized gas inside and outside the PDR. Previous observational (Hoopes \& Walterbos, 2003) and theoretical work (Wood \& Mathis, 2004) shows that radiation escaping the H II region has a softer spectrum due to absorption and re-emission processes in the surrounding

gas. This would indicate a decrease in the $y^{+}=N\left({ }^{4} \mathrm{He}^{+}\right) / N\left(\mathrm{H}^{+}\right)$ionic abundance ratio by number outside the PDR. We derive $y^{+}$using

$$
y^{+}=\frac{T_{\mathrm{L}}\left({ }^{4} \mathrm{He}^{+}\right) \Delta V\left({ }^{4} \mathrm{He}^{+}\right)}{T_{\mathrm{L}}\left(\mathrm{H}^{+}\right) \Delta V\left(\mathrm{H}^{+}\right)},
$$

where $T_{\mathrm{L}}\left({ }^{4} \mathrm{He}^{+}\right)$and $T_{\mathrm{L}}\left(\mathrm{H}^{+}\right)$are the line strengths of $\mathrm{He}$ and $\mathrm{H}$, respectively, and $\Delta V\left({ }^{4} \mathrm{He}^{+}\right)$and $\Delta V\left(\mathrm{H}^{+}\right)$are the corresponding FWHM line widths (Peimbert et al., 1992). If He was not detected, we use upper limits of $T_{\mathrm{L}}\left({ }^{4} \mathrm{He}^{+}\right)=3 \times \mathrm{rms}$ and $\Delta V\left({ }^{4} \mathrm{He}^{+}\right)=0.84 \Delta V\left(\mathrm{H}^{+}\right)$to find $y^{+}$. Here, the constant 0.84 is the average line width ratio $\Delta V\left({ }^{4} \mathrm{He}^{+}\right) / \Delta V\left(\mathrm{H}^{+}\right)$from our $\mathrm{RRL}$ data. This value is in agreement with Wenger et al. (2013), who found $\Delta V\left({ }^{4} \mathrm{He}^{+}\right) / \Delta V\left(\mathrm{H}^{+}\right)=0.77 \pm 0.25$, averaged over 54 individual H II regions.

Results for the ionic abundance ratio $y^{+}$, electron temperatures (see Section 2.4.5), and dust temperatures (see Section 2.4.6) are summarized in Table 2.2. Listed are the Galactic longitude and latitude, the location of the observation with respect to the PDR ("on" for on the PDR, "in" for inside the PDR, "out" for outside the PDR), the ionic abundance ratio, the LTE electron temperature, and the estimated dust temperature (see next section). All uncertainties are 1 $\sigma$. In Figure 2.6, we 
show $y^{+}$along the EW direction. Though not located along the EW direction, we also include the source $\mathrm{SE}$ at $(\ell, b)=\left(111.603^{\circ}, 0.762^{\circ}\right)$ in the plot. This position is surrounded by a faint lobe of radio continuum emission outside the PDR similar to the eastern locations.

Table 2.2: Derived Properties

\begin{tabular}{lcclccccc}
\hline Source & $\ell$ & $b$ & PDR & $y^{+}$ & $\sigma y^{+}$ & $\begin{array}{c}T_{\mathrm{e}} \\
(\mathrm{K})\end{array}$ & $\begin{array}{c}\sigma T_{\mathrm{e}} \\
(\mathrm{K})\end{array}$ & $\begin{array}{c}T_{\mathrm{d}} \\
(\mathrm{K})\end{array}$ \\
\hline E5 & 111.649 & 0.813 & out & $<0.149$ & $\ldots$ & $\ldots$ & $\ldots$ & 20.6 \\
E4 & 111.627 & 0.813 & out & $<0.045$ & $\ldots$ & $\ldots$ & $\ldots$ & 20.6 \\
E3.5 & 111.616 & 0.813 & out & $<0.066$ & $\ldots$ & $\ldots$ & $\ldots$ & 20.5 \\
E3 & 111.605 & 0.813 & out & 0.091 & 0.008 & 10,550 & 180 & 19.3 \\
E2 & 111.582 & 0.813 & on & 0.083 & 0.002 & 8,330 & 50 & 21.8 \\
E1 & 111.559 & 0.813 & in & 0.092 & 0.003 & 8,250 & 60 & 22.8 \\
C & 111.544 & 0.813 & in & 0.086 & 0.002 & 7,890 & 50 & 23.9 \\
W1 & 111.530 & 0.813 & in & 0.088 & 0.001 & 7,520 & 20 & 26.4 \\
W2 & 111.507 & 0.813 & on & 0.091 & 0.003 & 9,440 & 60 & 25.8 \\
SE & 111.603 & 0.762 & out & $<0.063$ & $\ldots$ & $\ldots$ & $\ldots$ & 19.8 \\
\hline Note - All uncertainties & are $\pm 1 \sigma$. & & & & &
\end{tabular}

The ionic abundance ratio $y^{+}$declines outside the PDR boundary but remains relatively constant inside. We find an average total ionic abundance ratio inside/on the PDR of $y^{+}=0.088 \pm 0.003$. Directly outside the eastern PDR boundary at a distance of $+0.061^{\circ}$ at position $\mathrm{E} 3, y^{+}$is $0.091 \pm 0.008$, and then decreases with increasing distance. The large upper limit at a distance of $+0.105^{\circ}$ at position E5 is a result of the low signal-to-noise ratio of the observation and does not necessarily indicate an actual increase of $y^{+}$. We estimate an upper limit on the average $y^{+}=$ 0.034 for all locations with He non-detections outside the PDR. The decline in $y^{+}$ with increasing distance suggests that the radiation field softens as the radiation passes through the PDR (Reynolds \& Tufte, 1995; Hoopes \& Walterbos, 2003; Wood \& Mathis, 2004; Roshi et al., 2012). Since at position E3, which is directly outside our $12 \mu \mathrm{m}$-defined PDR boundary, $y^{+}$is comparable to that inside, it may indicate 


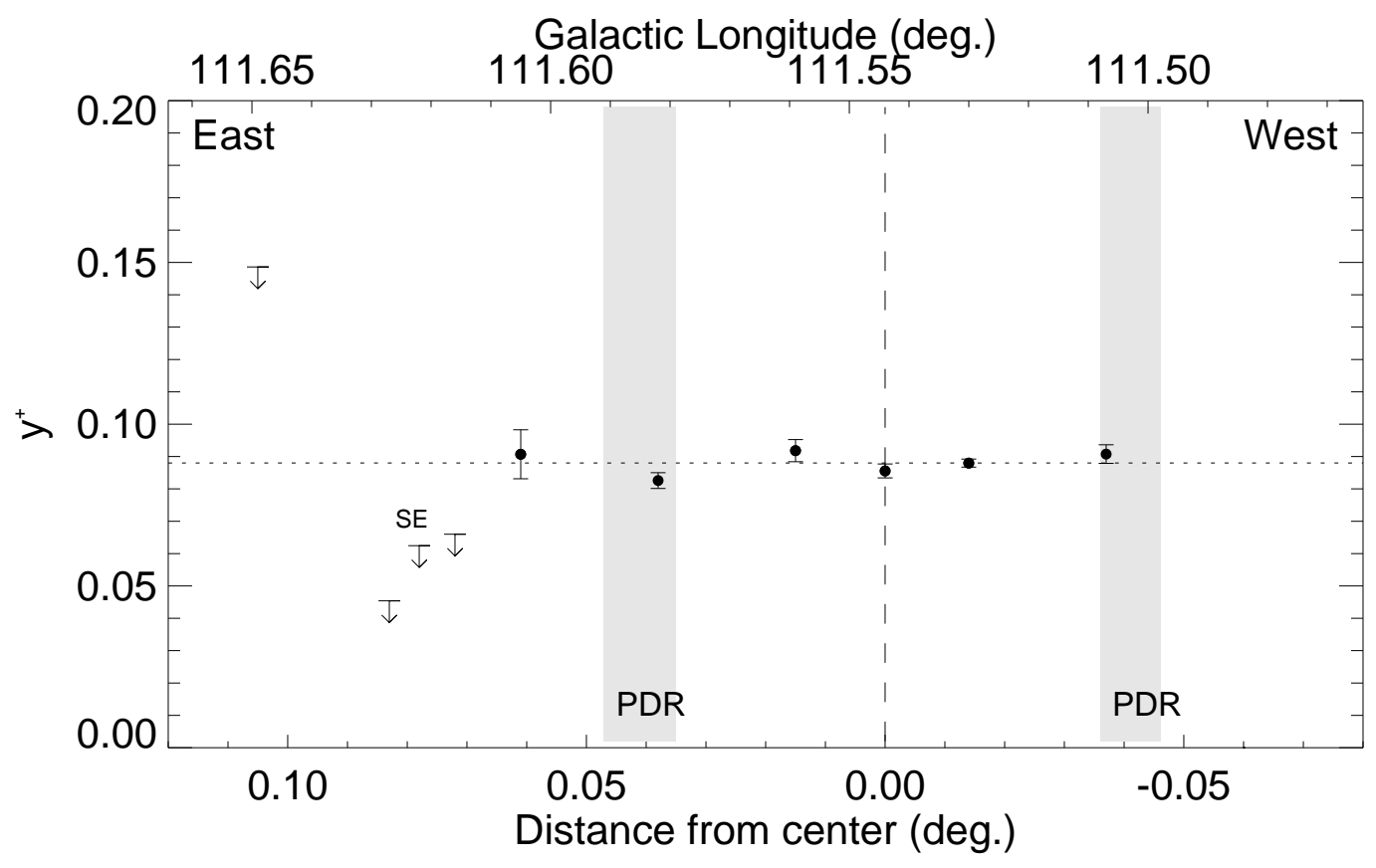

Figure 2.6: The ionic abundance ratio $y^{+}$as a function of distance from the central location. The dotted horizontal line shows the average $y^{+}=0.088$ inside the PDR. All observed RRLs, with the exception of position SE (marked in the figure), were taken along the EW direction at a Galactic latitude of $b=0.813^{\circ}$. The x-axis and meaning of the shaded regions are the same as in Figure 2.4. Error bars are $\pm 1 \sigma$. These measurements are consistent with a softening of the stellar radiation field outside the PDR. 
that the PDR extends slightly further to the east than our $12 \mu$ m-definition.

\subsubsection{Carbon RRLs}

Carbon RRL emission is primarily observed in PDRs (see Hollenbach \& Tielens, 1999). Because of its lower first ionization potential $(11.2 \mathrm{eV})$, carbon can be ionized by softer radiation than $\mathrm{H}(13.6 \mathrm{eV})$ and $\mathrm{He}(24.6 \mathrm{eV})$. This softer radiation is better able to pass through the HII region and into the PDR surrounding it. Figure 2.7 shows the observed intensity of the carbon RRL, $T_{\mathrm{L}}(\mathrm{C})$, along the EW direction, again including the position SE. If $\mathrm{C}$ was not detected, an upper limit defined by 3 times the observed rms is used. Here, we use the original spectral resolution of $1.86 \mathrm{~km} \mathrm{~s}^{-1}$ as the typical $\mathrm{C}$ line profile is more narrow compared to $\mathrm{H}$ and He. As expected, we find the largest carbon RRL intensities near the PDR boundaries of the H II region.

The observed intensity of the line at position W2 on the western PDR is $\sim 19 \mathrm{mK}$, which is two times higher than that detected at any other pointing. It is probably no coincidence that the W2 position falls on the PDR. We hypothesize that this PDR is dense and is absorbing the C-ionizing photons. Position E2 on the weaker eastern PDR also has a detected carbon line, although only at an intensity of $\sim 6 \mathrm{mK}$, comparable to values found both inside and outside the H II region.

\subsubsection{Electron Temperature}

The electron temperature $T_{\mathrm{e}}$ is an important physical parameter of $\mathrm{H}$ II regions that can be used to assess the efficiency of cooling processes. There is debate in the literature about the relationship between the electron temperature inside and outside the H II region PDR. While previous observations of H II regions have found a fairly homogeneous spatial $T_{\mathrm{e}}$ distribution (see Roelfsema et al., 1992; Adler et al., 1996;

Krabbe \& Copetti, 2002; Rubin et al., 2003), an increase in scatter has been observed at the edges of NGC 346 (Oliveira et al., 2008). For the well-studied Orion nebula, the results are inconclusive, with optically-derived values of $T_{\mathrm{e}}$ the same inside and 


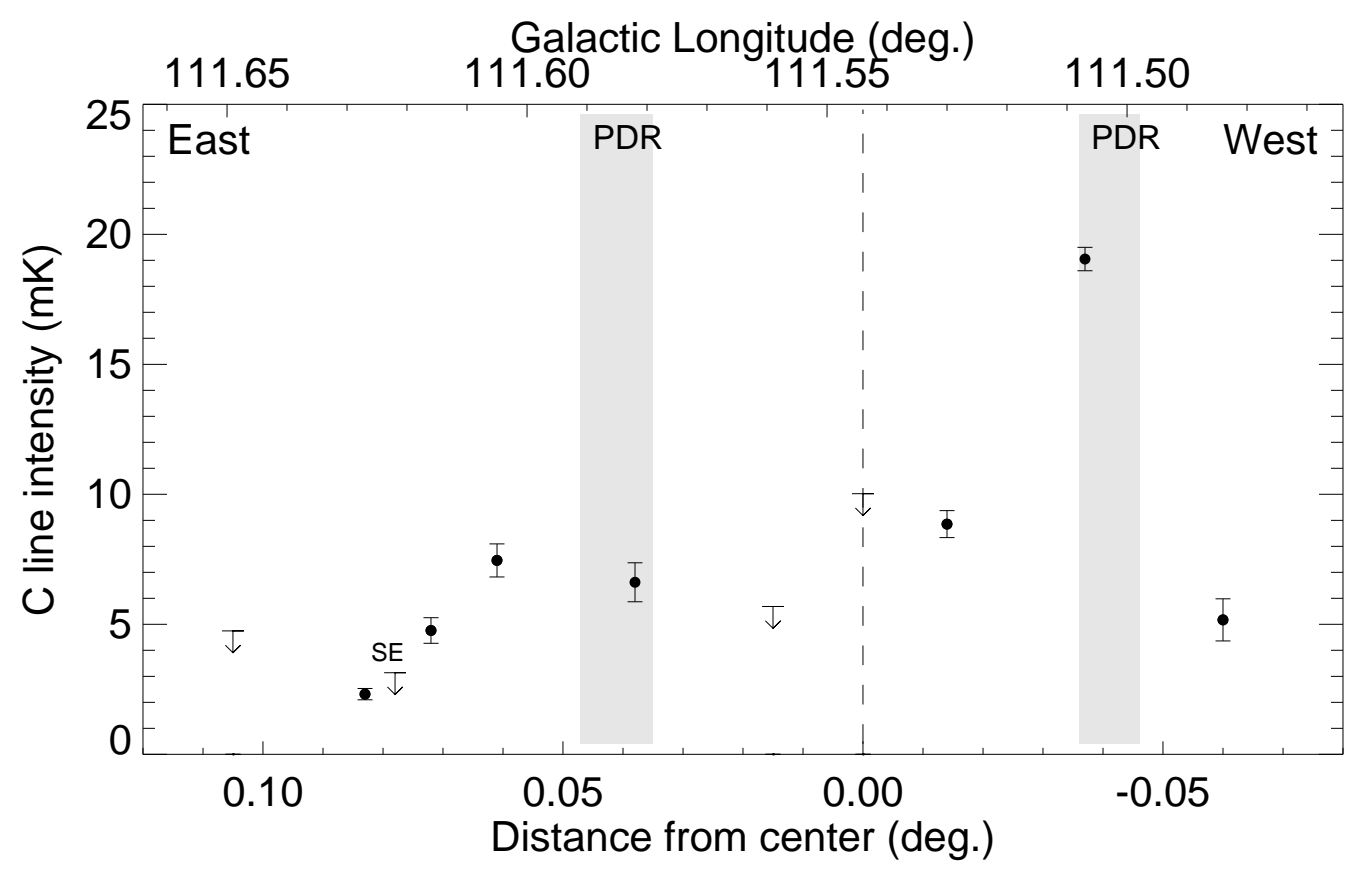

Figure 2.7: Carbon line intensity as a function of distance from the central location in Galactic coordinates. Shown are all C RRLs measured in the EW direction at a Galactic latitude of $b=0.813^{\circ}$ with the addition of position SE (marked). The $\mathrm{x}$-axis and meaning of the shaded regions are the same as in Figure 2.4. Error bars are $\pm 1 \sigma$. 
outside the PDR (McCall, 1979) or different findings for different species (Walter, 1994; Weilbacher et al., 2015). Recently, Wilson et al. (2015) found a decrease in the radio-derived electron temperature with angular offset for Orion A.

We calculate the electron temperature assuming local thermal equilibrium (LTE) for all RRL positions where a He line could be detected, using

$$
\left(\frac{T_{\mathrm{e}}^{*}}{\mathrm{~K}}\right)=\left\{7103.3\left(\frac{\nu_{\mathrm{L}}}{\mathrm{GHz}}\right)^{1.1}\left[\frac{T_{\mathrm{C}}}{T_{\mathrm{L}}\left(\mathrm{H}^{+}\right)}\right] \times\left[\frac{\Delta V\left(\mathrm{H}^{+}\right)}{\mathrm{km} \mathrm{s}^{-1}}\right]^{-1} \times\left[1+y^{+}\right]^{-1}\right\}^{0.87}
$$

where $\nu_{\mathrm{L}}=8.9 \mathrm{GHz}$ is the average frequency of our Hn $\alpha$ recombination lines, $T_{\mathrm{C}}$ is the continuum antenna temperature, $T_{\mathrm{L}}$ is the $\mathrm{H}$ line antenna temperature, $\Delta V\left(\mathrm{H}^{+}\right)$ is the FWHM line width, and $y^{+}$is the ionic abundance ratio found by Eq. 2.2 (see Quireza et al., 2006). We derive errors in $T_{\mathrm{e}}$ by propagating errors in $T_{\mathrm{C}}, T_{\mathrm{L}}, \Delta V\left(\mathrm{H}^{+}\right)$, and $y^{+}$via Eq. 2.3. Here, the uncertainty of the continuum antenna temperature $\sigma T_{\mathrm{C}}$ is due to random temperature fluctuations found by taking the rms of an Off-source location in the map close to NGC 7538. Uncertainties of all line parameters are based on the Gaussian fits used to characterize the line.

We show the derived electron temperature values as a function of distance from the central location in Figure 2.8. We find an average temperature of $T_{\mathrm{e}}=7890 \pm$ $300 \mathrm{~K}$ within the PDR of the region. The peak of the radio emission (position W1) has $T_{\mathrm{e}}=7520 \pm 20 \mathrm{~K}$. Our E3 position outside the PDR is much hotter with $T_{\mathrm{e}}=$ $10050 \pm 180 \mathrm{~K}$, similar to the W2 position on the PDR $\left(T_{\mathrm{e}}=9440 \pm 60 \mathrm{~K}\right)$. The electron temperature at the E2 position that is also located on the PDR, however, is closer to the average value inside with $T_{\mathrm{e}}=8330 \pm 50 \mathrm{~K}$. It appears that $T_{\mathrm{e}}$ remains roughly constant within the PDR but may increase outside the PDR boundary. Previous work at the same observing frequencies reports average electron temperatures for NGC 7538 of $8230 \pm 40 \mathrm{~K}$ (Quireza et al., 2006) and $8483 \pm 51 \mathrm{~K}$ (Balser et al., 2011) which are slightly higher than our results. This is likely due to uncertainties in the observed continuum antenna temperatures. Quireza et al. (2006) assigns a quality factor of "B" to the continuum data of NGC 7538, whereas Balser et al. (2011) gives a quality factor of only "C" ("A" is highest quality). Additionally, observations at slightly different 


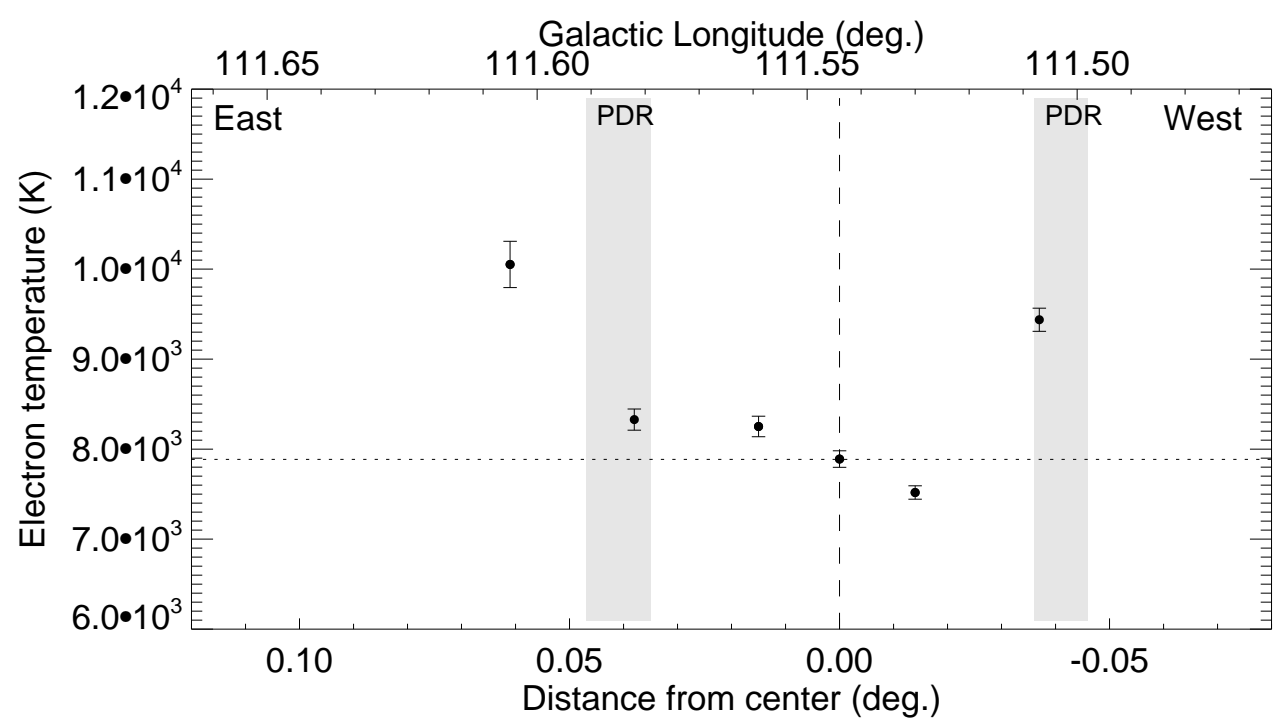

Figure 2.8: LTE electron temperature $T_{\mathrm{e}}$ as a function of distance from the central location. The x-axis and meaning of the shaded regions are the same as in Figure 2.4. The dotted horizontal line shows the average $T_{\mathrm{e}}=7890 \mathrm{~K}$ inside the PDR. The electron temperature remains relatively constant inside the PDR, but we detect significantly higher values of $T_{\mathrm{e}}$ on and outside the PDRs. Error bars are $\pm 1 \sigma$.

locations may result in differences in the calculated electron temperatures. Quireza et al. $(2006)$ observed at $(\ell, b)=\left(111.53^{\circ}, 0.82^{\circ}\right)$ and Balser et al. $(2011)$ observed at $(\ell, b)=\left(111.525^{\circ}, 0.816^{\circ}\right)$. These positions are offset from our W1 position by $25^{\prime \prime}$ and $22^{\prime \prime}$, respectively.

\subsubsection{Dust Properties}

Higher dust temperatures outside the PDR may be correlated with the locations along the PDR that have significant fractions of escaping radiation. Anderson et al. (2012b) and Anderson et al. (2015) show that for the H II region RCW 120 the locations of dust temperature enhancements outside the PDR are correlated with "holes" in the PDR. These results indicate that radiation can escape through these holes to heat the ambient medium. This effect has yet to be investigated in detail for other H II regions, although it may be related to the extended radio continuum emission around ultra-compact H II regions observed by Kim \& Koo (2001). 
We use Herschel Space Observatory data at 160, 250, 350, and $500 \mu \mathrm{m}$ from the HOBYS key-time program (Motte et al., 2010) to derive dust temperature and column density maps of NGC 7538. The HOBYS data of NGC 7538 were first shown in Fallscheer et al. (2013). Here we use a similar method to create these maps by fitting grey-body models pixel-by-pixel, after regridding to the common spatial resolution of the $350 \mu \mathrm{m}$ data (see Anderson et al., 2012b). In the fits we assume a dust emissivity index $\beta=2$. We subtract an offset from all data at the position $(\ell, b)=\left(113.271^{\circ}, 0.425^{\circ}\right)$, as this location is relatively devoid of emission. To convert to column density, we assume a dust-to-gas ratio of 100, and an opacity $\kappa_{\nu}=0.1(\nu / 1000 \mathrm{GHz})^{\beta}$.

We show the dust temperature map of NGC 7538 in Figure 2.9. The contour in the left panel of Figure 2.9 is at $23 \mathrm{~K}$, as this temperature best highlights the features of the dust temperature distribution near the PDR. We see higher dust temperatures to the north and east of the PDR compared to the west and south, which may suggest that leaking radiation is heating the ambient material. Within the second PDR to the east (defined in Figure 2.3) we detect higher dust temperatures of $>22 \mathrm{~K}$ compared to $\sim 20 \mathrm{~K}$ outside. This indicates that the second PDR is an additional barrier to the propagation of photons from the region further into the ISM. The point-like temperature enhancement to the south of the region is associated with the infrared source IRS 9 and is not related to dust heating by NGC 7538 itself.

The contours in the right panel of Figure 2.9 are of column density of the neutral gas. The dust temperature is higher in directions where the column density along the PDR is lower, namely toward the north and east. There is a small region of high column density on the eastern PDR. This region is presumably leading to the two "lobes" of radio emission seen in this direction (Figure 2.1). Toward the south and west there is high column density material along the PDR that is spatially correlated with lower dust temperatures. 

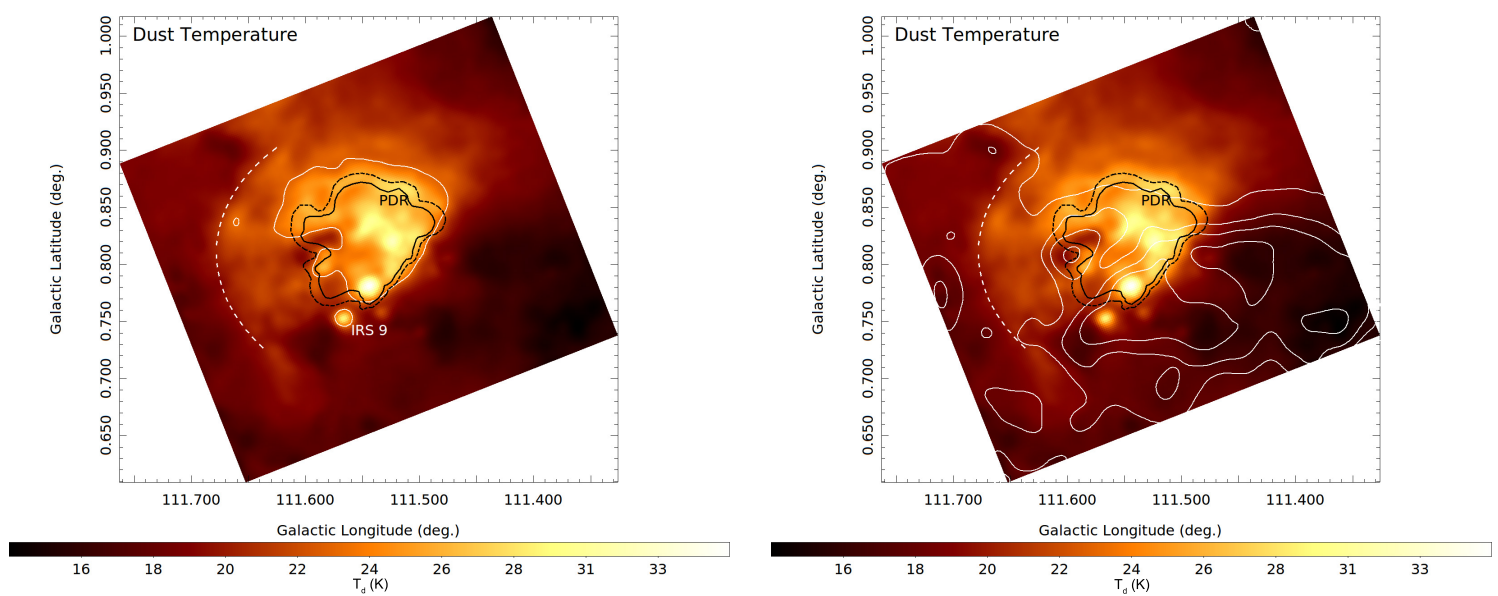

Figure 2.9: Left: Dust temperature map of NGC 7538, derived from Herschel data (see Anderson et al., 2012b). The solid white outline indicates the boundary of $23 \mathrm{~K}$ dust temperature (hotter dust inside). The inner/outer PDR boundaries are indicated by the solid/dashed black lines marked "PDR". The second PDR is indicated by the dashed white line to the east. Significant temperature enhancements are observed outside the PDR in the northern and eastern part of the region, possibly due to leakage through the PDR. These temperature enhancements to the east are confined to the second PDR boundary. The point-like enhancement to the south of the region is associated with the infrared source IRS 9 and is not related to dust heating by NGC 7538 itself. Right: Same image as left, except that the white contours are of column density. Contour levels are at 3, 6, 10, and $20 \times 10^{21} \mathrm{~cm}^{-2}$. Locations along the PDR of high column density are associated with low dust temperatures, and vice versa, showing how unimpeded radiation can heat the local ambient medium. 


\subsection{Discussion}

Studies of external galaxies suggest that $30-70 \%$ of the emitted hydrogenionizing photons escape from H II regions into the ISM (e.g., Oey \& Kennicutt, 1997; Zurita et al., 2002; Giammanco et al., 2005; Pellegrini et al., 2012). In our Galaxy, however, information on radiation leaking from HII regions is sparse. Furthermore, Galactic studies are often contaminated by confusion since we reside in the Galactic plane (see Anderson et al., 2015b). Here we use the Outer Galaxy H II region NGC 7538 as a case study since there is less confusion along the line of sight to this source.

The detection of radio continuum and RRL emission outside the PDR of NGC 7538 hints that the ionizing photons from H II regions could be responsible for some of the diffuse RRL emission detected in the plane of the Galaxy (Anderson et al., 2011; Anderson et al., 2015b). The intensity of this emission outside the PDR, however, decreases rapidly with increasing distance. To quantify this decrease, we fit a power law of the form $T_{\mathrm{L}}[\mathrm{mK}]=T_{\mathrm{L}, 0} d^{\alpha}$ to the $\mathrm{H}$ RRL intensity outside the PDR. Here, the model parameter $T_{\mathrm{L}, 0}$ is the antenna temperature value at a distance of $1 \mathrm{pc}$ from the center of the region at $(\ell, b)=\left(111.544^{\circ}, 0.813^{\circ}\right), d$ is the distance in pc from the center, and $\alpha$ is the power law index. Since the antenna temperature on the PDR varies with direction, we have to fit each direction separately. We only fit RRL intensities towards the east of the region due to a lack of data points in the other directions. We find $\alpha=-3.8$, indicating a very steep decrease with distance. These results are shown in the top panel of Figure 2.10.

We repeat this analysis for the complex W43 region (bottom panel of Figure 2.10) using RRL data from Anderson et al. (2015b) for which the emission has been identified as "diffuse" (i.e., not from within the PDR of a discrete H II region). These data were taken and reduced using the same methodology employed here and so are directly comparable to our data for NGC 7538. We use all RRL detections within $2^{\circ}$ of W43 that have velocities between 80 and $120 \mathrm{~km} \mathrm{~s}^{-1}$ and assume the maser parallax distance for W43 is $5.5 \mathrm{kpc}$ (Zhang et al., 2014). We split our analysis 

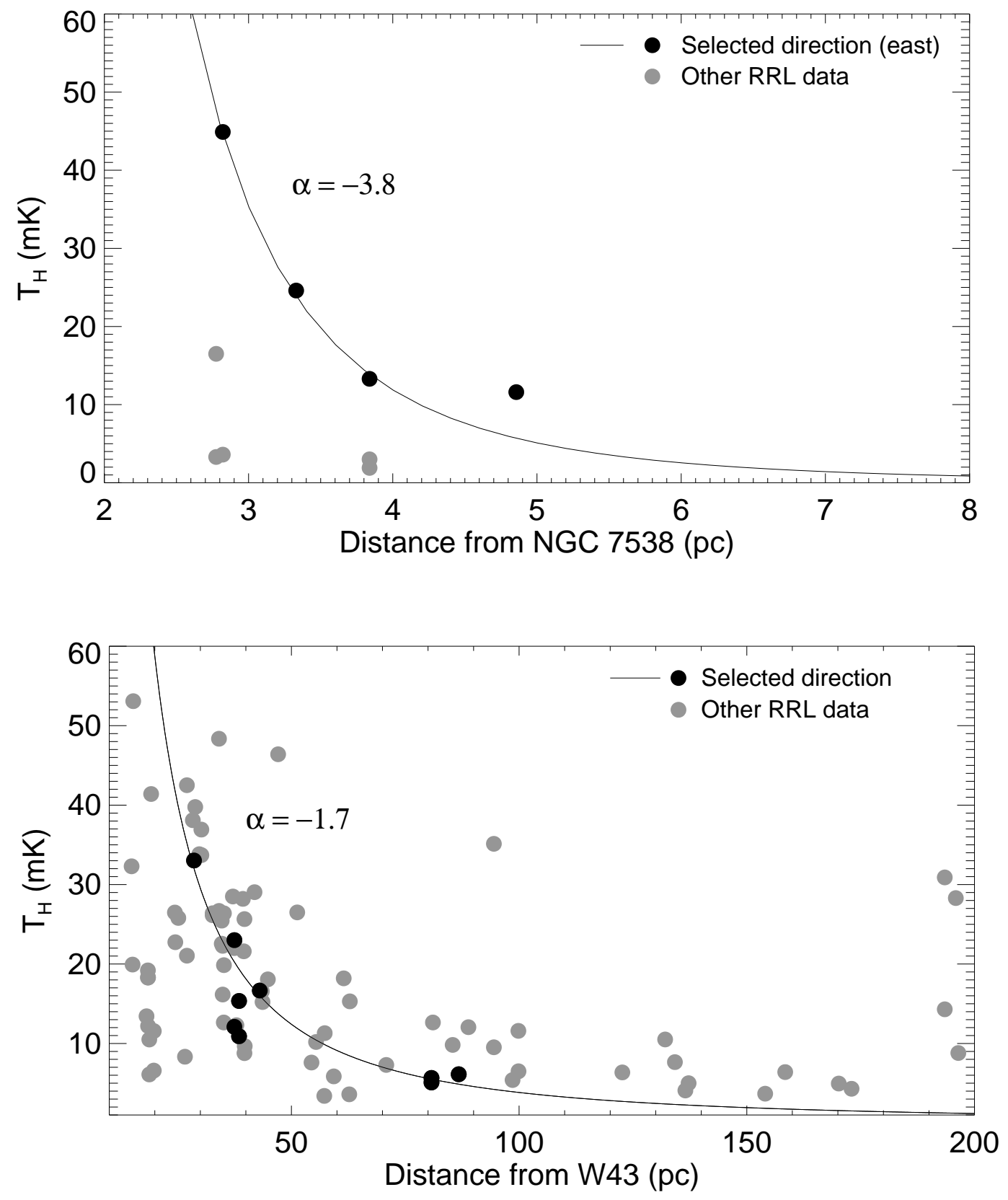

Figure 2.10: The RRL intensity outside the PDR of NGC 7538 (top) and W43 (bottom) as a function of distance from the nominal center. Due to insufficient data points in the other directions, we only fit data from the east direction for NGC 7538. Due to the asymmetrical nature of W43, we must fit the data separately for different directions from the region. The shown fit for W43 represents a $10^{\circ}$ sector centered at $295^{\circ}$ from Galactic north. The emission from NGC 7538 decreases more rapidly than that from W43 as indicated by the power-law index $\alpha$ in the figures. 
into sectors of $10^{\circ}$ opening angle around W43 and fit each separately. This allows us to detect spatial asymmetries of the diffuse emission around W43 similar to the analysis of NGC 7538 above. The power law fits range from $\alpha=-0.26$ to $\alpha=-3.01$, with an average of $\alpha=-0.54$, indicating that the decrease in diffuse RRL intensity for W43 is significantly less steep than that for NGC 7538. A representative power law fit (the $10^{\circ}$ sector centered at $295^{\circ}$ from Galactic north) for W43 is shown in Figure 2.10 for illustration purposes.

What would lead to the difference in power law indices? W43 is much more luminous than NGC 7538. Smith et al. (1978) list a flux density of 86.5 Jy for W43 at $5 \mathrm{GHz}$. Given their respective Heliocentric distances, and assuming that both regions are optically thin, this implies that W43 is $\sim 20$ times more luminous than NGC 7538. Zurita et al. (2000) show that, in a model suggested by Beckman et al. (2000), leaking radiation from very luminous H II regions is sufficient to ionize the diffuse gas. The model, based on the slope of $\mathrm{H} \alpha$ luminosity functions of $\mathrm{H}$ II regions, assumes that high luminosity HII regions are primarily density-bounded. This is consistent with their data on a sample of six spiral galaxies and would allow a large number of ionizing photons to escape into the surrounding medium. If the extended emission around W43 is due to W43 itself, the shallow power law may imply that this is the case here. As a result, a significant fraction of the leaked radiation would be able to ionize the diffuse gas. Along the W43 sight line, however, it is difficult to disentangle the possible contribution from the numerous H II regions. We therefore cannot be sure that all the diffuse radiation is due to W43 itself. Another model, proposed by Anantharamaiah (1986), suggests that the extended emission could be due to low-density envelopes of size $\sim 100$ pc around individual compact H II regions. Due to the high population of H II regions around W43, our line of sight could cross many of these envelopes. Roshi \& Anantharamaiah (2001) argue that this model is in good agreement with RRL observations at $327 \mathrm{MHz}$ in the Galactic plane.

The steep power law of NGC 7538 shows the difficulty in detecting radio emission far from such compact H II regions. We see from Figure 2.10 that $5 \mathrm{pc}$ from the center the fitted RRL intensity is just $\sim 5 \mathrm{mK}$. Furthermore, there is clearly a second PDR 
seen toward the east, visible at $12 \mu \mathrm{m}$ in Figure 2.3. The zone interior to this second PDR has a higher dust temperature with a sharp boundary (see Figure 2.9). This implies that in this direction the radiation is leaking through the primary PDR. This radiation, however, is being further attenuated by the secondary PDR and is not truly escaping the region.

Our results cast doubt on whether compact H II regions like NGC 7538 can be responsible for the diffuse ionized gas detected in the inner Galaxy. Since this is a case study of only one such region, however, the broader implications are unclear. While giant H II region complexes such as W43 seem to leak a significant fraction of their ionizing radiation into the ISM, more study is required on individual regions. Larger H II regions in the Outer Galaxy such as W3/W4/W5 may be suitable targets for future observations as there is little confusion along the line of sight.

\subsection{Summary}

We observed the HII region NGC 7538 with the NRAO Green Bank Telescope (GBT) in $\sim 8.7 \mathrm{GHz}$ continuum and recombination line emission to investigate the escape of radiation through the PDR into the local interstellar medium. This leaked radiation from H II regions could be an important source of photons that maintain the ionization of the WIM.

Using the radio continuum intensity, we estimate the leaked radiation fraction from NGC 7538. We first visually define the PDR using WISE $12 \mu \mathrm{m}$ infrared images and sum the pixel values of the radio continuum map inside and outside the PDR to find the total intensity of NGC 7538. We calculate a total intensity of $17.3 \mathrm{Jy}$ inside the region which would roughly be expected from a single $\mathrm{O} 5 \mathrm{~V}$ star. Using the ratio of the outside to total radio continuum intensity, we compute the percentage of leaked emission to be $f_{\mathrm{R}}=15 \pm 5 \%$ in the plane of the sky. We also use $\mathrm{H} \alpha$ data from IPHAS to repeat the calculation and find $f_{\mathrm{H} \alpha}=31 \pm 10 \%$. Due to the

three-dimensional geometry of the region, these numbers represent lower limits on the total leaked emission. 
The leaking radiation is not found everywhere around the region, but rather is mostly in the north and east. These are also the directions where we identify dust temperature enhancements, and locations along the PDR of decreased column density. This shows how, due to a non-uniform PDR, radiation can escape the region in some directions to heat the ambient medium while it is confined in other directions.

RRL measurements of NGC 7538 are consistent with a decrease in the ionic abundance number ratio $y^{+}$with increasing distance outside the PDR and an increased carbon line emission in the PDR, suggesting a softening of the radiation field. We compute an average LTE electron temperature of $7890 \pm 300 \mathrm{~K}$ inside the region, which is marginally lower than that derived in previous work (Balser et al., 1999; Quireza et al., 2006).

The RRL intensity from NGC 7538 decreases rapidly outside the PDR. This decrease is much steeper than that seen for the high mass star formation complex W43. Furthermore, the existence of an additional PDR boundary further from NGC 7538 implies that the "leaked" emission is not able to traverse large distances. This seems to imply that maintaining the WIM might rather be caused by giant H II region complexes regions such as W43 than "normal" compact H II regions like NGC 7538. 


\section{Chapter 3}

\section{Diffuse Ionized Gas in the Milky Way Disk}

\subsection{Abstract}

We analyze the diffuse ionized gas (DIG) in the first Galactic quadrant from $\ell=18^{\circ}$ to $40^{\circ}$ using radio recombination line (RRL) data from the Green Bank Telescope. These data allow us to distinguish DIG emission from H II region emission and thus study the diffuse gas essentially unaffected by confusion from discrete sources. We find that the DIG has two dominant velocity components, one centered around $100 \mathrm{~km} \mathrm{~s}^{-1}$ associated with the luminous HII region W43, and the other centered around $45 \mathrm{~km} \mathrm{~s}^{-1}$ not associated with any large $\mathrm{HII}$ region. Our analysis suggests that the two velocity components near W43 may be caused by non-circular streaming motions originating near the end of the Galactic bar. At lower Galactic longitudes, the two velocities may instead arise from gas at two distinct distances from the Sun, with the most likely distances being $\sim 6 \mathrm{kpc}$ for the $100 \mathrm{~km} \mathrm{~s}^{-1}$ component and $\sim 12 \mathrm{kpc}$ for the $45 \mathrm{~km} \mathrm{~s}^{-1}$ component. We show that the intensity of diffuse Spitzer GLIMPSE $8.0 \mu \mathrm{m}$ emission caused by excitation of polyaromatic hydrocarbons (PAHs) is correlated with both the locations of discrete HII regions and the intensity of the RRL emission from the DIG. This implies that the soft ultra-violet photons responsible for creating the infrared emission have a similar origin as the harder ultra-violet photons required for the RRL emission. The $8.0 \mu \mathrm{m}$ emission increases with RRL intensity but flattens out for directions with the most intense RRL emission, suggesting that PAHs are partially destroyed by the energetic radiation field at these locations.

The research contained in this chapter was published as Luisi et al. (2017), ApJ, 849, 117.

Contributing authors: L. D. Anderson, D. S. Balser, T. V. Wenger, T. M. Bania. 


\subsection{Introduction}

First proposed by Hoyle \& Ellis (1963), the warm interstellar medium (WIM) is a widespread component of the interstellar medium (ISM) with density $\sim 0.1 \mathrm{~cm}^{-3}$ and temperatures from 6000 to $10000 \mathrm{~K}$ (see Haffner et al., 2009, and references therein). At the upper end of this temperature range, the WIM is nearly fully ionized, with a hydrogen ionization ratio $n\left(\mathrm{H}^{+}\right) / n\left(\mathrm{H}^{0}\right) \geq 13$ (Reynolds et al., 1998). Thus, the WIM is also known as the "Diffuse Ionized Gas" (DIG). Despite its low density, 80-90\% of the total free-free emission in our Galaxy is thought to come from the DIG.

Though the exact mechanisms are still unknown, it is believed that the DIG maintains its ionization from O-type stars, whose UV radiation leaks out of the H II regions surrounding them and into the ISM (Reynolds, 1984; Ferguson et al., 1996; Zurita et al., 2002). Murray \& Rahman (2010) confirmed that a large number of ionizing photons are leaking from H II regions. Anderson et al. (2015) derived an ionizing radiation leaking fraction of $\sim 25 \%$ for the bubble H II region RCW 120 using $\mathrm{H} \alpha$ data at $656 \mathrm{~nm}$. They also showed that the photodissociation region (PDR) surrounding the H II region has distinct "holes" through which photons can escape into the ISM. This suggests that PDRs are generally not homogeneous. Recently, we showed that the non-uniform PDR surrounding the compact H II region NGC 7538 allows radiation to escape preferentially along a single direction (Luisi et al., 2016). We calculated a leaking fraction $f_{R}=15 \pm 5 \%$ of the radio continuum emission. This leaking emission appears spatially confined within an additional, more distant PDR boundary around NGC 7538 and thus seems to only affect the local ambient medium. Results suggest, however, that giant H II regions such as W43 may have a much larger effect in maintaining the ionization of the DIG and despite their small numbers may be the dominant source of ionizing radiation in the ISM (see Zurita et al., 2000).

Together with HII regions and PDRs, the DIG is a major source of radio recombination line (RRL) emission. Consequently, RRL observations have been used to map its spatial and velocity distribution. Compared to studies of optical emission lines, specifically H $\alpha$ (e.g., the WHAM survey, Haffner et al., 1999), RRL observa- 
tions have the advantage of essentially being free from extinction due to interstellar dust. Their disadvantage is reduced sensitivity, restricting RRL detections to gas with higher emission measure than that traced by $\mathrm{H} \alpha$. The fully-sampled $1.4 \mathrm{GHz} \mathrm{RRL}$ survey of Alves et al. (2010, 2012, 2015) mapped the plane of the Galaxy at a spatial resolution of 14'.4. They were, however, unable to distinguish the contributions from discrete H II regions and the DIG for most sight lines. The observing method of the fully-sampled SIGGMA RRL survey (Liu et al., 2013) partially filters out the emission from the DIG. Finally, Roshi \& Anantharamaiah (2001) observed the Galactic plane in RRLs near $327 \mathrm{MHz}$ from $-28^{\circ}<\ell<89^{\circ}$. Despite the low resolution of $\sim 2^{\circ}$, they obtain an upper limit of $12,000 \mathrm{~K}$ for the electron temperature of the gas and suggest that the emission originates from low-density ionized gas forming H II region envelopes.

With the emergence of high-sensitivity RRL surveys, the DIG has been serendipitously detected in observations of discrete H II regions (see Anderson et al., 2015b, and references therein). In the Green Bank Telescope H II Region Discovery Survey (GBT HRDS, see Bania et al., 2010; Anderson et al., 2011) we identified multiple RRL velocity components in $\sim 30 \%$ of all observed targets. This fraction is too large to be caused by multiple discrete H II regions along the line of sight (see Anderson et al., 2015b). We thus infer that the RRL emission at these locations is usually composed of emission from a discrete source and emission from the DIG (Anderson et al., 2015b).

Here, we use data from past observations (Anderson et al., 2011; Anderson et al., 2015b) and previously unpublished data for directions either known to be devoid of discrete H II regions, or in directions where the H II region emission can be distinguished from that of the DIG (see $\S 3.3$ for details on how we distinguish between these two components). This gives us an irregularly-spaced grid of pointings, for which we can extract the intensity and velocity of only the DIG. The advantage of our strategy is that the beam size is relatively small $\left(82^{\prime \prime}\right)$ compared with typical spacings between discrete H II regions so the emission at each pointing is not contaminated with H II region emission. The disadvantage of course is that the $(\ell, b)$-space is not 
fully sampled. By distinguishing the emission from discrete H II regions and the DIG, our data allows us to essentially filter out H II region emission entirely and map only the diffuse component. This gives us an advantage over previous RRL surveys (Liu et al., 2013; Alves et al., 2015) as these are at least partially contaminated by H II region emission. With this analysis we are able to investigate the relationship between discrete H II regions and the diffuse gas, and test our hypothesis that large H II regions are dominant in maintaining the ionization of the DIG (Zurita et al., 2000; Luisi et al., 2016).

\subsection{DIG RRL Emission}

Our RRL emission data were taken with the Auto-Correlation Spectrometer (ACS) on the National Radio Astronomy Observatory Green Bank Telescope (GBT). We observed a total of 254 directions between $\ell=18^{\circ}$ and $40^{\circ}$ and $|b|<1^{\circ}$ which yielded 379 sets of line parameters for the DIG. Our data come from two previously published sources, Anderson et al. (2011) and Anderson et al. (2015b), and one previously unpublished source (see below). Anderson et al. (2011) contains directions coincident with H II regions, as defined by $8 \mu \mathrm{m}$ Spitzer GLIMPSE emission, for which the diffuse gas velocity can be distinguished from the H II region velocity (these data include 98 pointings with 116 sets of diffuse line parameters). The process of distinguishing the diffuse gas velocities from H II region velocities is described in Anderson et al. (2015b). We use previous GBT observations, analyze the derived electron temperature for each velocity component, and search for the molecular emission or carbon recombination lines associated with one RRL component. Sight lines that that do not pass within the $8 \mu \mathrm{m}$-defined H II region PDR are always considered "diffuse." Anderson et al. (2015b) also includes such directions devoid of discrete H II regions which allows us to directly sample the DIG without confusion (135 pointings; 237 sets of line parameters).

We also incorporate observations taken near the giant H II region W43 (21 pointings; 26 sets of diffuse line parameters) which we have not published previously. Here, 
we use our HRDS data to distinguish the diffuse gas velocity from the H II region velocity. If the observed direction is spatially coincident with a known H II region, we assume that the velocity component closest to the HII region velocity is due to the H II region itself. We summarize these data in Table 3.1, which lists the source, the Galactic longitude and latitude, the hydrogen line intensity, the FWHM line width, the local standard of rest (LSR) velocity, and the rms noise in the spectrum, including all corresponding $1 \sigma$ uncertainties of the Gaussian fits. For directions with multiple velocity components detected along the line of sight, the source names are given additional letters, "a," "b," or "c," in order of decreasing peak line intensity. Velocity components that are due to discrete H II regions are marked with an asterisk in the table and are not used for our data analysis. For each observed direction, we simultaneously measured $7 \mathrm{Hn} \alpha$ RRL transitions in the $9 \mathrm{GHz}$ band, $\mathrm{H} 87 \alpha$ to $\mathrm{H} 93 \alpha$, using our standard techniques (Bania et al., 2010; Balser et al., 2011; Anderson et al., 2011), and averaged all spectra together to increase the signal-to-noise ratio using TMBIDL $^{1}$ (Bania et al., 2014). We assume that the brightest line emission from the DIG is due to hydrogen and fit a Gaussian model to each line profile. We use the line intensities, full width at half maximum (FWHM) values, and LSR velocities derived from the Gaussian fits for all further analysis.

\subsection{Discussion}

\subsubsection{The Galactic Location of the DIG}

Over the longitude range considered here, the DIG emission is concentrated near two velocities, $45 \mathrm{~km} \mathrm{~s}^{-1}$ and $100 \mathrm{~km} \mathrm{~s}^{-1}$ (Figure 3.1). This suggests that within our observed Galactic longitude range the DIG itself is located at two distinct distances, assuming that the diffuse gas in each velocity range can be assigned a single distance. We summarize the DIG emission properties in Table 3.2.

\footnotetext{
${ }^{1}$ V7.1, see https://github.com/tvwenger/tmbidl.git.
} 


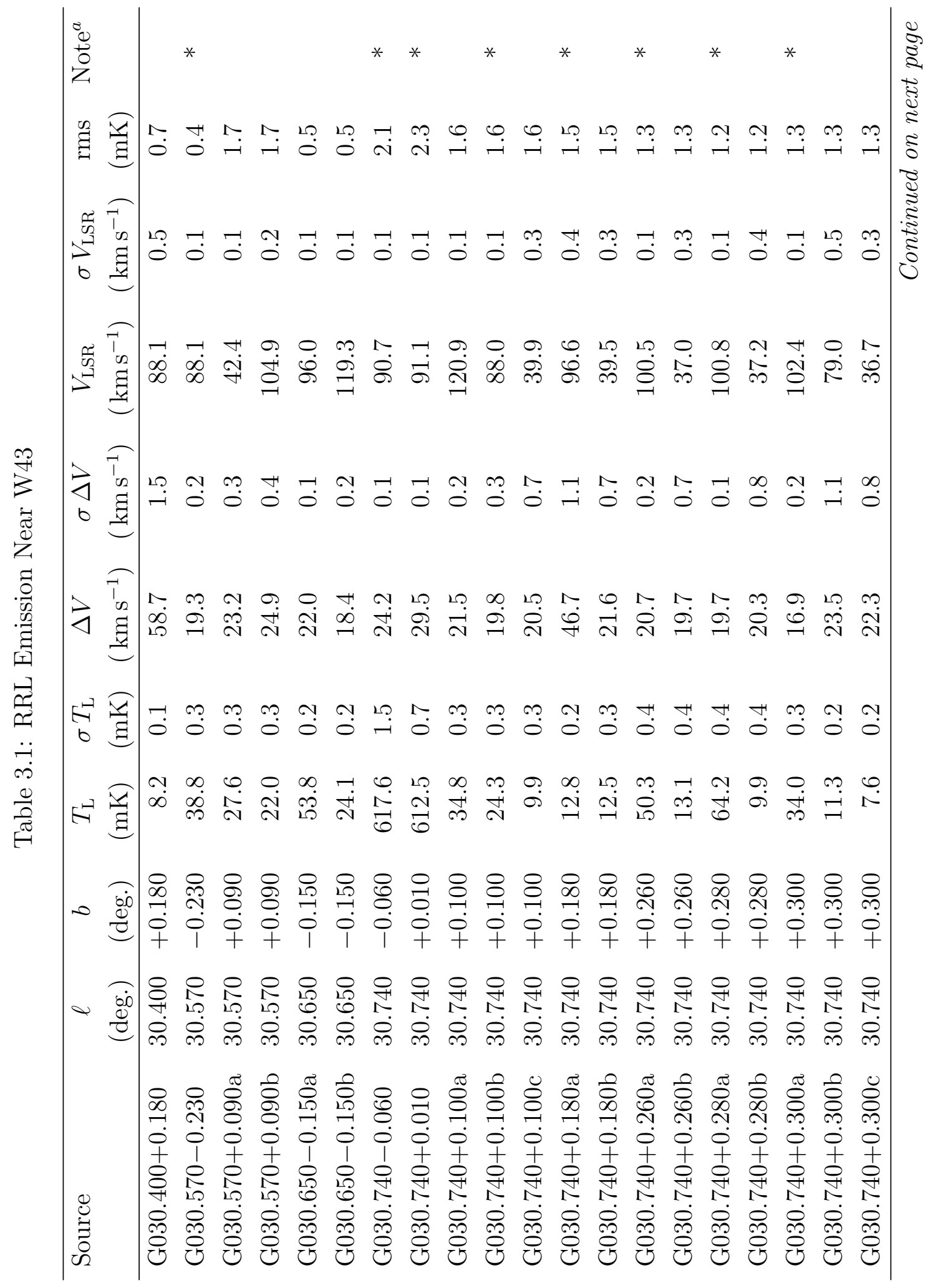




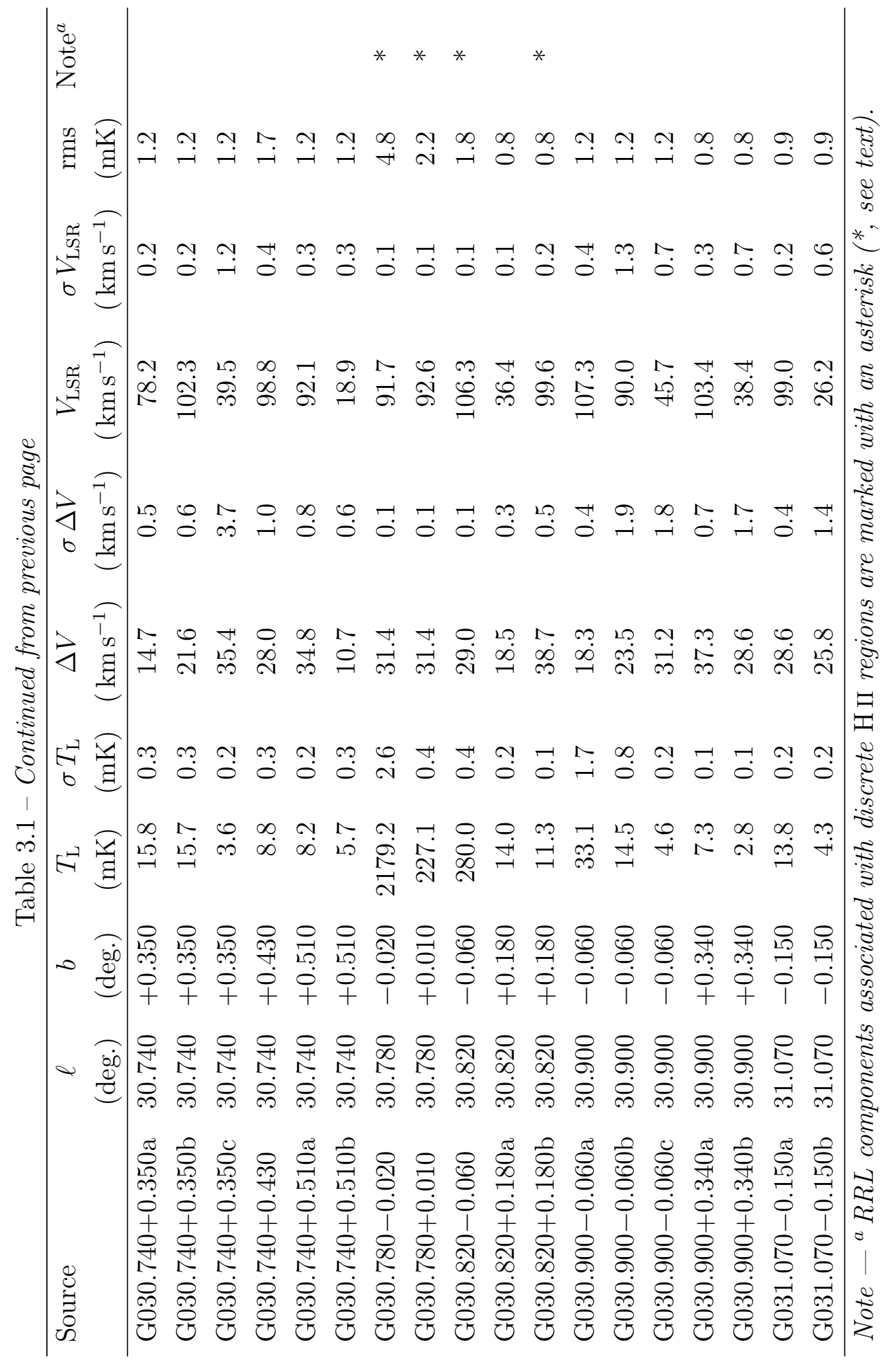


Just as for the discrete sources, however, this diffuse gas also suffers from the kinematic distance ambiguity (KDA). For H II regions, the KDA can often be resolved using the H I emission/absorption (H IE/A) method (Kuchar \& Bania, 1994; Kolpak et al., 2003; Anderson et al., 2012a). The HIE/A method uses the fact that in the Galactic plane H I is found toward most directions and velocities. Cold H I foreground to the HII region is seen in absorption; if the HII region is at the near kinematic distance, H I absorption features will be present at velocities up to the H II regions velocity. However, if the region is at the far distance, it will show absorption features up to the tangent point velocity. Absorption between the HII region velocity and the tangent point velocity therefore indicates that the region is at the far kinematic distance. Unfortunately, we cannot use the H IE/A method for the DIG, both because it is faint and also because of the difficulty in finding a suitable "off" position. Only massive stars can produce ionizing photons energetic enough to create and maintain the DIG (e.g., Reynolds, 1984). We can therefore potentially determine the kinematic distance for the diffuse gas by associating it with massive star formation tracers that have their KDA resolved: massive H II regions, molecular gas, and cold H I.

Below, we attempt to find the distance to the two observed velocity components of the DIG by resolving their KDA. In $§ 3.4 .1 .1$ and $\S 3.4 .1 .2$ we assume that each velocity component can be assigned a single distance from the Sun. In $§ 3.4 .1 .3$ we explore the possibility of the two observed velocity components being due to interacting gas clouds at the same distance from the Sun.

\subsubsection{The $45 \mathrm{~km} \mathrm{~s}^{-1}$ Gas Component}

The KDA leads to two possible distance ranges for each velocity range. The $45 \mathrm{~km} \mathrm{~s}^{-1}$ gas could be at either $1.7-3.7 \mathrm{kpc}$ or $10.6-12.7 \mathrm{kpc}$, if we assume $(\ell, b)=$ $\left(30^{\circ}, 0^{\circ}\right)$, and use the Reid et al. (2014) rotation curve (see Table 3.2).

Assuming that the DIG is maintained by massive stars, we can use the ionization rate of HII regions as a tracer to determine the distance to the DIG. In the range $\ell=18^{\circ}$ to $40^{\circ}$, there are $205 \mathrm{H}$ II regions with velocities between 25 and $65 \mathrm{~km} \mathrm{~s}^{-1}$, and 127 of these have kinematic distance ambiguity resolutions (KDARs; Anderson 


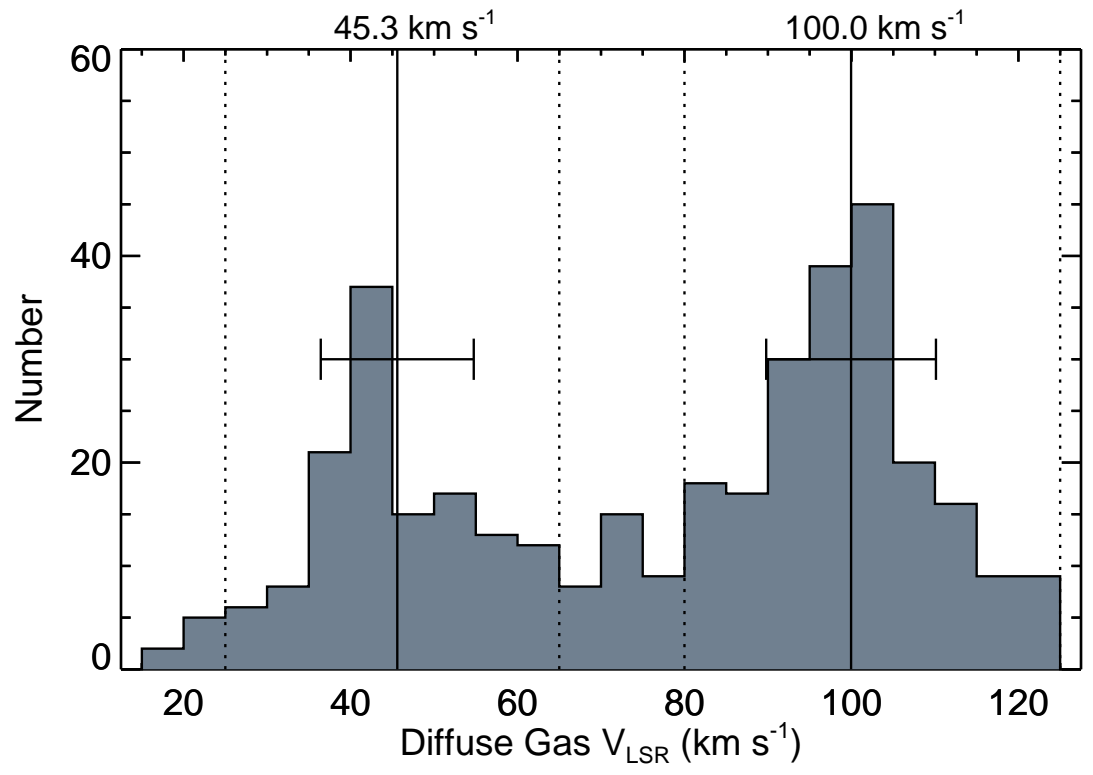

Figure 3.1: Velocity distribution of the DIG. The LSR velocities are derived from Gaussian fits to our RRL data. The dotted lines are defined by eye and show the velocity ranges that contain the majority of the diffuse gas velocities: the $45 \mathrm{~km} \mathrm{~s}^{-1}$ component at $25-65 \mathrm{~km} \mathrm{~s}^{-1}$ and the $100 \mathrm{~km} \mathrm{~s}^{-1}$ component at $80-125 \mathrm{~km} \mathrm{~s}^{-1}$. The solid lines and error bars indicate the mean velocities within these ranges and their standard deviations. 
et al., 2014, The WISE Catalog of Galactic H II Regions, Version 1.4 ${ }^{2}$ ). The total radio flux density of the 94 regions at the far kinematic distance is $10.84 \mathrm{Jy}$, whereas the total flux density of the 33 regions at the near kinematic distance is only 1.09 Jy.

Table 3.2: DIG Parameters

\begin{tabular}{lcc}
\hline Velocity Range & $45 \mathrm{~km} \mathrm{~s}^{-1}$ & $100 \mathrm{~km} \mathrm{~s}^{-1}$ \\
\hline Number of RRL components (total) & 128 & 211 \\
Number of RRL components (on $)^{a}$ & 33 & 63 \\
Number of RRL components (off) & 95 & 148 \\
\hline Mean velocity $\left(\mathrm{km} \mathrm{s}^{-1}\right)$ & 45.3 & 100.0 \\
Median velocity $\left(\mathrm{km} \mathrm{s}^{-1}\right)$ & 43.0 & 99.4 \\
Std. Dev. velocity $\left(\mathrm{km} \mathrm{s}^{-1}\right)$ & 9.2 & 10.1 \\
\hline Mean $T_{\mathrm{A}}(\mathrm{mK})$ & 12.5 & 16.8 \\
Median $T_{\mathrm{A}}(\mathrm{mK})$ & 9.8 & 13.6 \\
Std. Dev. $T_{\mathrm{A}}(\mathrm{mK})$ & 9.6 & 11.2 \\
\hline Near distance $(\mathrm{kpc})$ & $1.7-3.7$ & $4.4-7.2$ \\
Far distance $(\mathrm{kpc})$ & $\sim 12$ & $\sim 6$ \\
Assumed distance $(\mathrm{kpc})$ & 172.8 & 246.0 \\
\hline Total integrated flux $(\mathrm{Jy})$ & 118.3 & 220.4 \\
Total integrated flux $(\mathrm{Jy})^{b}$ &
\end{tabular}

Note — " "on" and "off" correspond to directions coincident with $\mathrm{H}$ II regions (on), and directions devoid of discrete $\mathrm{H}$ II regions (off). ${ }^{b}$ From Alves et al. (2015).

We estimate the ionization rate for each region using our HRDS data (Rubin, 1968; Anderson, 2010) by

$$
N_{\mathrm{ly}} \approx 4.76 \times 10^{48}\left(\frac{S_{\nu}}{\mathrm{Jy}}\right)\left(\frac{T_{\mathrm{e}}}{\mathrm{K}}\right)^{-0.45}\left(\frac{\nu}{\mathrm{GHz}}\right)^{0.1}\left(\frac{d}{\mathrm{kpc}}\right)^{2}
$$

where $N_{\text {ly }}$ is the ionization rate, the number of emitted Lyman Continuum ionizing photons per second, $S_{\nu}$ is the radio flux density of the $\mathrm{H}$ II region, $T_{\mathrm{e}}$ is the electron

\footnotetext{
${ }^{2}$ see http://astro.phys.wvu.edu/wise/
} 
temperature, $\nu=1.4 \mathrm{GHz}$ is the observed frequency (see Anderson et al., 2011), and $d$ is the distance to the region. We assume a constant $T_{\mathrm{e}}=10^{4} \mathrm{~K}$ and sum the contribution for each individual region to find the total $N_{\text {ly }}$ for H II regions at the far and near kinematic distance. This estimate yields $N_{\text {ly }}=1.06 \times 10^{50} \mathrm{~s}^{-1}$ for the far distance and only $N_{\mathrm{ly}}=5.80 \times 10^{47} \mathrm{~s}^{-1}$ for the near distance. This suggests that most of the DIG near $45 \mathrm{~km} \mathrm{~s}^{-1}$ is also at the far kinematic distance.

There is also over twice as much total CO gas at the far kinematic distance for clouds in the velocity range 25 to $65 \mathrm{~km} \mathrm{~s}^{-1}$ compared to the near distance. The average near GRS cloud CO luminosity from Roman-Duval et al. (2009) in units of $10^{4} \mathrm{~K} \mathrm{~km} \mathrm{~s}^{-1} \mathrm{pc}^{-2}$ is 0.23 with a standard deviation of 0.31 , while it is 1.4 with a standard deviation of 1.7 for the far GRS clouds. The total CO luminosity for the near clouds is $3.4 \times 10^{5} \mathrm{~K} \mathrm{~km} \mathrm{~s}^{-1} \mathrm{pc}^{-2}$, while it is $8.0 \times 10^{5} \mathrm{~K} \mathrm{~km} \mathrm{~s}^{-1} \mathrm{pc}^{-2}$ for the far GRS clouds. This again supports the $45 \mathrm{~km} \mathrm{~s}^{-1}$ DIG being at the far kinematic distance, if it is indeed associated with the molecular gas traced by $\mathrm{CO}$ emission that will continue to form massive stars.

Finally, we investigate the location of the cold H I gas using the H I E/A method. Only cold H I foreground to a radio continuum source will cause H I absorption, assuming that H I self-absorption is negligible. The H I spectrum toward an extragalactic radio continuum source can show absorption for all $\mathrm{H}$ I along the line of sight, while for Galactic sources the H I spectrum can only show absorption up to the source velocity. Comparing the H I spectra toward nearby extragalactic and Galactic H II region pairs can therefore tell us about the $\mathrm{HI}$ distribution. If cold $\mathrm{HI}$ gas is foreground to the H II region, we expect to see absorption in both spectra. Cold H I beyond the H II region, however, will only show absorption in the spectrum toward the extragalactic source.

Here we use the Very Large Array Galactic Plane Survey (VGPS; see Stil et al., 2006) spectral line data to compare the HIE/A spectrum for three HII regions $\left(\mathrm{G} 24.47+0.49, \mathrm{G} 24.81+0.10\right.$, and W43) with velocities near $100 \mathrm{~km} \mathrm{~s}^{-1}$. All three have nearby (within $\sim 40^{\prime}$ ) extragalactic radio continuum sources. Figure 3.2 shows the difference between on- and off-target directions for the H II regions and extragalac- 
tic radio sources, where the on- and off-positions are separated by $6^{\prime}$. The H II regions are located either foreground or background to the $45 \mathrm{~km} \mathrm{~s}^{-1}$ gas, depending on their KDARs. As a result, H I gas at velocities showing extragalactic absorption which is not present in the H II region spectra should be background to the H II region. This analysis implies that most of the $\mathrm{H}$ I below $50 \mathrm{~km} \mathrm{~s}^{-1}$ near W43 is at the far kinematic distance. The first H I spectrum pair (G24.47+0.49) extracted near $\ell=24^{\circ}$ shows partial absorption near $45 \mathrm{~km} \mathrm{~s}^{-1}$ that is inconsistent with the absorption features seen in the second pair near $\ell=24^{\circ}(\mathrm{G} 24.81+0.10)$. Therefore, we can not assign a single distance to the $\mathrm{HI}$ near the $\ell=24^{\circ}$ region. These results are somewhat ambiguous, however, since the separation between the line of sight towards the HII regions and the extragalactic continuum sources are probing different H I volumes.

Since both the total H II region ionization rate and the fraction of molecular gas are greater at the far distance, we favor the conclusion that most of the $45 \mathrm{~km} \mathrm{~s}^{-1}$ diffuse gas is at its far kinematic distance of $\sim 12 \mathrm{kpc}$ as well. This is a simplified assumption and does not take into account the existence of additional gas at the other distance.

\subsubsection{The $100 \mathrm{~km} \mathrm{~s}^{-1}$ Gas Component}

The possible distance range for the $100 \mathrm{~km} \mathrm{~s}^{-1}$ gas is $4.4-10.0 \mathrm{kpc}$ for $(\ell, b)=$ $\left(30^{\circ}, 0^{\circ}\right)$. Because the molecular gas and massive star formation for the $80-100 \mathrm{~km} \mathrm{~s}^{-1}$ $(\ell, b, v)$ locus is associated with W43 (e.g., Nguyen Luong et al., 2011) at a distance of $5.49_{-0.34}^{+0.39} \mathrm{kpc}$ (Zhang et al., 2014), we assume throughout the remainder of this paper that the $100 \mathrm{~km} \mathrm{~s}^{-1}$ DIG is at a distance of $\sim 6 \mathrm{kpc}$. Recently, Langer et al. (2017) observed the DIG along 18 lines of sight between $\ell=30^{\circ}$ and $32^{\circ}$ using the [C II] $158 \mu \mathrm{m}$ and [N II] $205 \mu \mathrm{m}$ fine structure lines. They find a strong line component near $\sim 115 \mathrm{~km} \mathrm{~s}^{-1}$ and argue that this component is due to DIG emission associated with the inner edge of the Scutum spiral arm tangency at a distance of $\sim 7 \mathrm{kpc}$. Even if our assumption that the gas is at the distance of $\sim 6 \mathrm{kpc}$ is poor, our conclusions below are largely unaffected. 

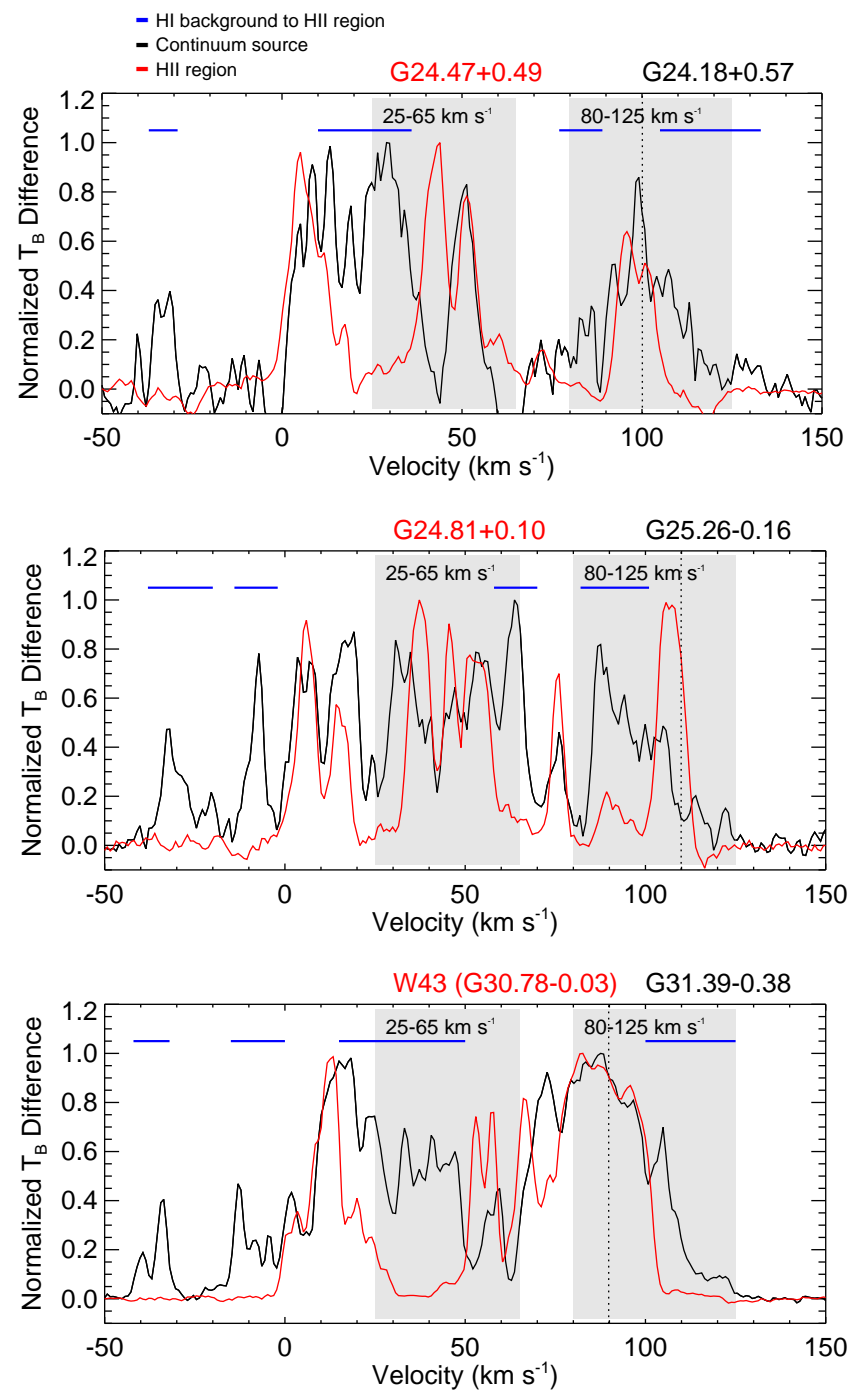

Figure 3.2: H I absorption spectra towards H II regions and extragalactic radio sources to investigate the KDAR of the DIG. The difference in VGPS main beam temperature, normalized by the maximum observed temperature, is shown between on- and off-target directions for H II regions (red curves) and extragalactic radio continuum sources (black curves). The top panel shows the H II region G24.47+0.49 and the extragalactic source G24.18+0.57, the middle panel shows $\mathrm{G} 24.81+0.10$ and the extragalactic source G25.26-0.16, and the bottom panel shows $\mathrm{W} 43$ at $(\ell, b) \simeq\left(30.8^{\circ}, 0.0^{\circ}\right)$ and the extragalactic source G31.39-0.38. The H II region RRL velocities are shown with dotted lines. Shaded regions indicate the two dominant velocity ranges of the DIG emission. Continuum source spectra showing absorption not present in the H II region spectra should be background to the H II region; these velocities are marked with horizontal blue lines at the top of each plot. This analysis implies that most of the $\mathrm{HI}$ below $50 \mathrm{~km} \mathrm{~s}^{-1}$ near W43 is at the far kinematic distance. 


\subsubsection{Interacting Gas Clouds?}

Our detection of the DIG in two separate velocity ranges suggests that each velocity range is primarily located at either its near or its far kinematic distance. If the two velocity components are indeed interacting, we would expect to observe an interaction signature between them. Such an interaction signature has been suggested by Beuther et al. (2012) for the ${ }^{13} \mathrm{CO}(2-1)$ emission near the W43 region, as well as for dense gas tracers like $\mathrm{N}_{2} \mathrm{H}^{+}$. In the Milky Way, however, this picture is further complicated by the vicinity of the $45 \mathrm{~km} \mathrm{~s}^{-1}$ component to the Galactic bar and the Scutum arm. Using an extragalactic counterpart to the W43 region, Beuther et al. (2017) argue that gas buildup near the bar/spiral arm interface, where W43 is located, is likely due to crossings between different orbit families. They posit that the observed velocities in the bar/spiral arm interface of NGC 3627 are primarily due to interacting gas clouds.

If we assume that the observed velocities toward the $\ell \sim 30^{\circ}$ region are due to interacting gas clouds at a single distance, we can use the method described by Beuther et al. (2017) to estimate the expected gas velocities observed along the line of sight and compare these with our observations. The simplest approximation assumes that the observed diffuse gas towards W43 is located at the tip of the Galactic bar, and that the two observed velocity signatures are due to the unperturbed, purely circular gas motion around the Galactic center and gas streaming motions along the bar, respectively. Using the Reid et al. (2014) rotation curve, we find a circular gas velocity $V_{C} \sim 230 \mathrm{~km} \mathrm{~s}^{-1}$ for the observed diffuse gas towards W43. This corresponds

to a velocity component along the line of sight of $91 \mathrm{~km} \mathrm{~s}^{-1}$, almost identical to the observed velocity of $89.8 \mathrm{~km} \mathrm{~s}^{-1}$ for W43 itself. The perturbed velocity component due to streaming motions can be described by determining the bar perturbation to the gravitational potential (see Sellwood \& Sánchez, 2010; Beuther et al., 2017). Since we only consider emission from the end of the bar, the radial streaming velocity 
component must go to zero, and the resultant azimuthal velocity component $v_{\phi}^{B}$ is

$$
v_{\phi}^{B} \sim\left(1-\frac{1-q_{\phi}^{2}}{4 q_{\phi}^{2}}\right) V_{C},
$$

where $q_{\phi}$ is the axial ratio of the bar potential. We use $1-q_{\phi} \simeq \frac{1}{3}(1-q)$ from Binney \& Tremaine (2008, p. 77), where $q=0.3-0.4$ is the axial ratio of the density distribution for the Milky Way bar (Bissantz \& Gerhard, 2002). We adopt $q=0.35 \pm 0.05$ and find that $v_{\phi}^{B}=0.84 \pm 0.02 V_{C}$. Observed along the line of sight, this corresponds to a velocity of $57 \pm 4 \mathrm{~km} \mathrm{~s}^{-1}$ which is near our observed $45 \mathrm{~km} \mathrm{~s}^{-1}$ velocity component.

Although the above method describes the observed velocity components near $\ell \sim 30^{\circ}$ fairly well, the assumption that the gas is located at the end of the Galactic bar breaks down when considering gas emission from the $\ell \sim 24^{\circ}$ region further within the bar where we observed a similar velocity distribution. To describe the kinematics of the gas at this location, we must include radial streaming motions along the bar (Sellwood \& Sánchez, 2010; Beuther et al., 2017) which can be estimated by

$$
v_{r}^{B} \sim \frac{2}{3}\left(1-\frac{1-q_{\phi}^{2}}{4 q_{\phi}^{2}}\right) V_{C} .
$$

We repeat the analysis above for the $\ell \sim 24^{\circ}$ region, and find an unperturbed velocity component along the line of sight of $96 \mathrm{~km} \mathrm{~s}^{-1}$, and a perturbed velocity component of $7 \pm 2 \mathrm{~km} \mathrm{~s}^{-1}$. In theory, shocks and turbulence could increase the latter to match our observed $45 \mathrm{~km} \mathrm{~s}^{-1}$ emission. While we can not quantify the amount of turbulence in the DIG directly, we can compare the observed hydrogen recombination line widths at the diffuse directions with the line widths of directions coincident with discrete $\mathrm{H}$ II regions. Assuming the same electron temperature, differences in line widths should trace the relative strength of turbulence between these directions. We find, however, no statistically significant difference of line widths between directions coincident with H II regions $\left(F W H M=24.5 \pm 6.4 \mathrm{kms}^{-1}\right)$ and our diffuse directions (FWHM = $23.7 \pm 8.8 \mathrm{~km} \mathrm{~s}^{-1}$ ). We show the corresponding FWHM line width distributions in Figure 3.3. This suggests that turbulence does not play a significant role in altering 


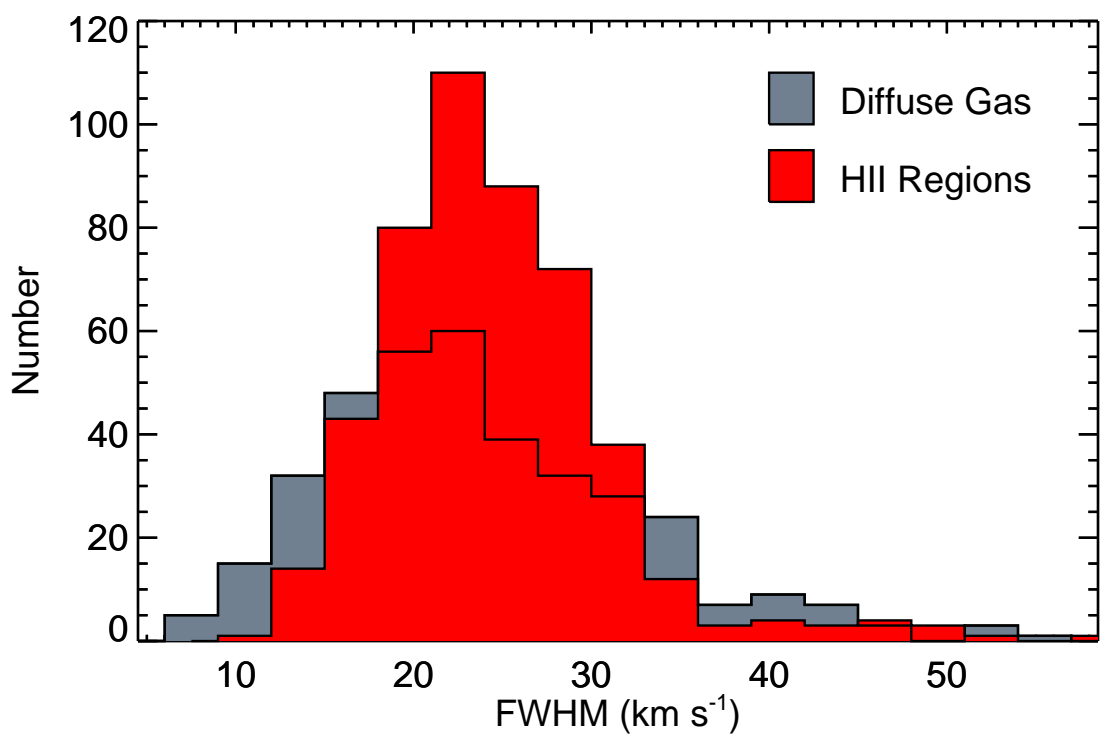

Figure 3.3: Distribution of FWHM line widths of directions coincident with discrete $\mathrm{H}$ II regions and the DIG. There is no significant difference between the average line widths of HII regions and the DIG (see text), indicating that turbulence does not play a major role in altering the observed velocity of the diffuse gas.

the observed velocity of the gas. As a result, the large difference of the derived $7 \mathrm{~km} \mathrm{~s}^{-1}$ velocity component to our observed $45 \mathrm{~km} \mathrm{~s}^{-1}$ emission makes it doubtful whether interacting gas clouds at a single distance near $\ell \sim 24^{\circ}$ could result in the observed velocity distribution.

Although the simple model discussed above suggests that interacting gas clouds cannot account for our observed data, a more thorough numerical analysis would be required to confirm this result. Renaud et al. (2013, 2015) developed a hydrodynamical simulation of a Milky Way-like galaxy which includes star formation and stellar feedback through photoionization, radiative pressure and supernovae. They find that the leading edges of bars are favorable locations for converging gas flows and shocks. A similar model, focusing on bar kinematics in particular, may provide more insight towards the interaction processes near the bar-spiral arm interface. 


\subsubsection{Intensity and Distribution of the DIG}

Our database of RRL parameters from the HRDS also allows us to investigate the spatial distribution of the DIG in the plane of the sky. Using our irregularly gridded data points, we examine the diffuse gas separately for the two velocities, $45 \mathrm{~km} \mathrm{~s}^{-1}$ and $100 \mathrm{~km} \mathrm{~s}^{-1}$. We create maps of the DIG in these two velocity ranges by interpolating the irregularly-spaced grid of 233 points to create pixels $6^{\prime}$ square. We do this by first performing a Delauney triangulation (using the IDL program "qhull") and then create an $(\ell, b)$ map of the RRL intensity from the DIG using inverse distance weighting (using the IDL program "griddata"). This method has the advantage that the maximum and minimum values in the interpolated surface can only occur at sample points. We assume that the top and bottom edges of the map $\left(b= \pm 1^{\circ}\right)$ have zero intensity to ensure that the emission is constrained in latitude. We show these images in Figure 3.4 for the two velocity ranges. We also show in Figure 3.4 the $1.4 \mathrm{GHz}$ H I Parkes All-sky survey RRL map (Alves et al., 2015) averaged over the velocity ranges of the $45 \mathrm{~km} \mathrm{~s}^{-1}$ and $100 \mathrm{~km} \mathrm{~s}^{-1}$ components for comparison. The green circles in Figure 3.4 show the locations of discrete H II regions cataloged by Anderson et al. (2014, Version 1.4) that are within the velocity range of interest, while the gray crosses show locations where the DIG was detected within the velocity range.

Using the same data set of RRL parameters, we explore the velocity distribution of the DIG in more detail. We create a longitude-velocity diagram of the DIG by interpolating between our grid points (Figure 3.5, top panel), and assume that the velocity edges of the diagram (at $0 \mathrm{~km} \mathrm{~s}^{-1}$ and $130 \mathrm{~km} \mathrm{~s}^{-1}$ ) have zero intensity so that the emission is constrained in velocity space. This assumption appears valid, since we did not detect any RRL components outside of this velocity range. In fact, our smallest and largest detected velocities at $18 \mathrm{~km} \mathrm{~s}^{-1}$ and $124 \mathrm{~km} \mathrm{~s}^{-1}$, respectively, are well within this range. For comparison, we also show a longitude-velocity diagram of ${ }^{12} \mathrm{CO}$ used to trace molecular clouds (Figure 3.5, bottom panel; data from Dame et al., 1987). 


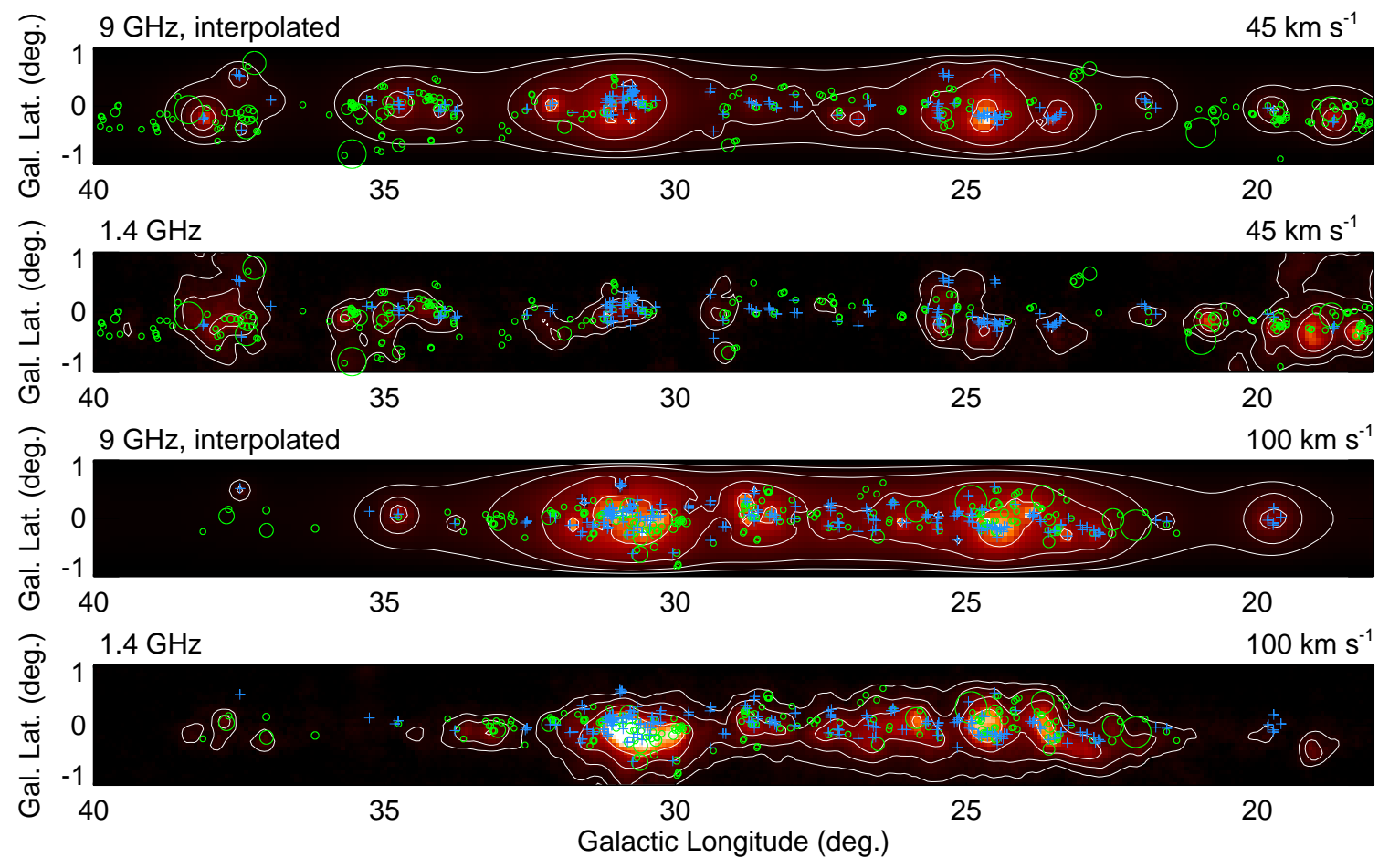

Figure 3.4: RRL emission over our observed longitude range. Top panel: Image of the diffuse gas emission for the $45 \mathrm{~km} \mathrm{~s}^{-1}$ velocity component. This image was made by interpolating our irregularly gridded $9 \mathrm{GHz}$ RRL data. The color scale shows the emission ranging linearly from 0 to $30 \mathrm{mK}$, and the contours are at 2, 4, 8, and $16 \mathrm{mK}$. The locations of the diffuse emission in this velocity range are marked by blue crosses, and green circles mark H II regions from the velocity range indicated. The H II region circle sizes and locations are from Anderson et al. (2014), with a minimum circle size of $0.1^{\circ}$ for better visibility. Second from top: Parkes $1.4 \mathrm{GHz}$ RRL survey map, integrated for the $45 \mathrm{kms}^{-1}$ velocity component (Alves et al., 2015). Unlike our interpolated image above, the $1.4 \mathrm{GHz}$ data includes emission from discrete H II regions. The color scale shows the emission ranging linearly from 0 to $200 \mathrm{mK}$, and the contours are at 15, 30, 60, and $120 \mathrm{mK}$. Third from top: Same as top panel, but for the $100 \mathrm{~km} \mathrm{~s}^{-1}$ velocity component. Bottom panel: Parkes RRL survey map for the $100 \mathrm{~km} \mathrm{~s}^{-1}$ velocity component (scale and contours equivalent to second panel from top). 

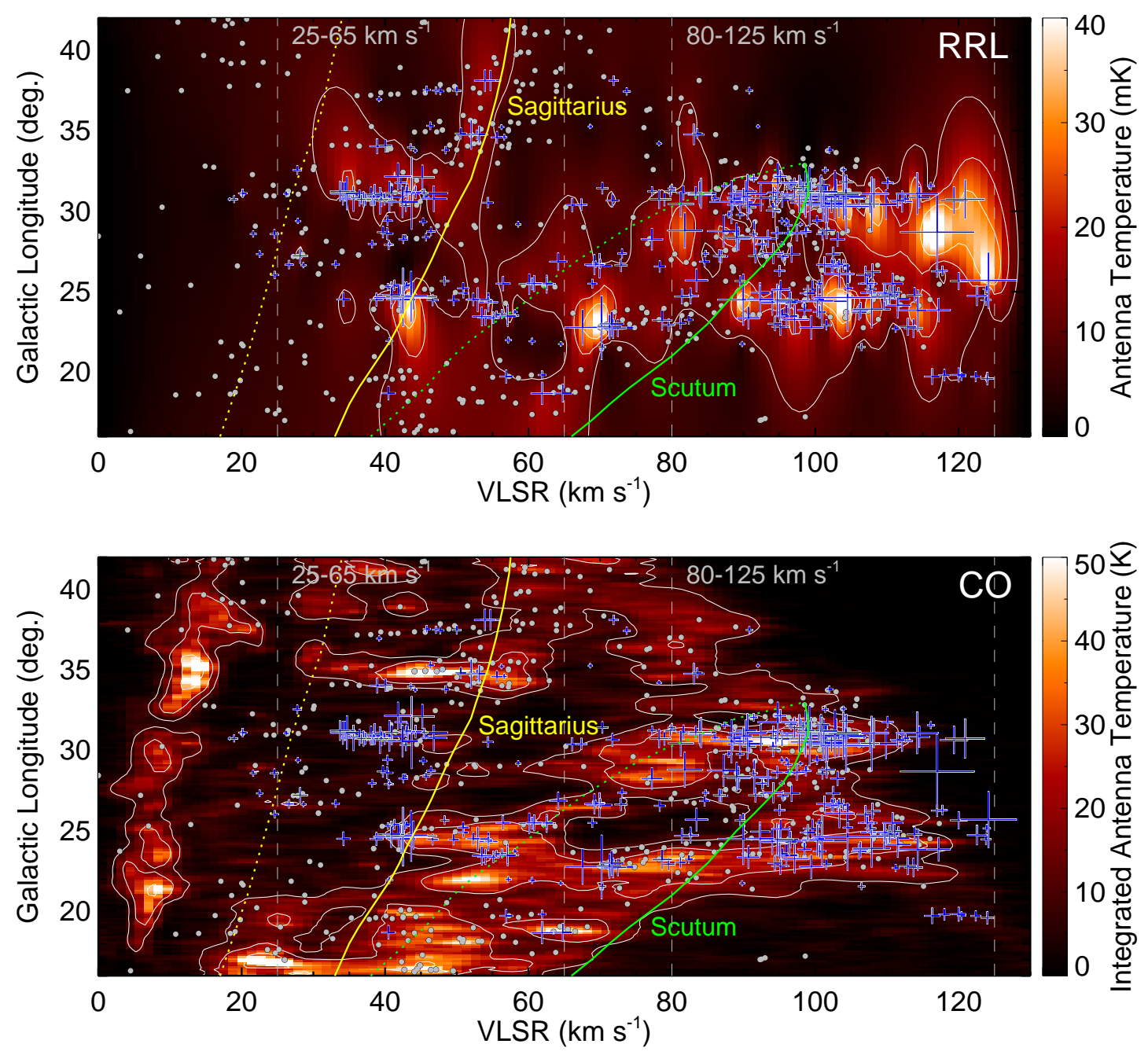

Figure 3.5: Top: Longitude-velocity diagram of the DIG. The white contours are at 10, 20, and $30 \mathrm{mK}$. The blue crosses denote the directions at which RRL spectra were taken and the cross sizes are proportional to the fitted H RRL intensities. The gray dots indicate discrete H II regions. The Sagittarius and Scutum spiral arms are traced in yellow and green, respectively (data from Reid et al., 2016). The dashed vertical lines indicate the two dominant velocity ranges: the $45 \mathrm{~km} \mathrm{~s}^{-1}$ component at $25-65 \mathrm{~km} \mathrm{~s}^{-1}$ and the $100 \mathrm{~km} \mathrm{~s}^{-1}$ component at $80-125 \mathrm{~km} \mathrm{~s}^{-1}$. Bottom: Same, for Galactic ${ }^{12} \mathrm{CO}$ emission, integrated in the range from $-1^{\circ}$ to $+1^{\circ}$ in latitude (see Dame et al., 1987). Contours are at 10, 20, and $40 \mathrm{~K}$. 


\subsubsection{The $45 \mathrm{~km} \mathrm{~s}^{-1}$ Gas Component}

The pixel-by-pixel correlation of RRL intensity at $45 \mathrm{~km} \mathrm{~s}^{-1}$ between our maps and the Alves et al. (2015) data is poor (see Figure 3.6, top panel). Our emission towards $\mathrm{W} 43$ near $\ell \sim 30^{\circ}$ and the $\ell \sim 24^{\circ}$ region is disproportionately large in the $45 \mathrm{~km} \mathrm{~s}^{-1}$ map, whereas we do not see strong emission near the map edge at $\ell \sim 19^{\circ}$. This may be due to interpolation errors between our sparse RRL pointings in this velocity and longitude range. Our low number of pointings may also be the cause of some of the more extended RRL emission between W43 and the $\ell \sim 24^{\circ}$ complex that is less pronounced in the Alves et al. (2015) data. This makes it challenging to distinguish between interpolation errors and actual diffuse gas below the Parkes 1.4 GHz RRL survey's sensitivity threshold for the undersampled regions in our maps. Additionally, the beam size of $\sim 14^{\prime}$ in the Alves et al. (2015) maps is too large to avoid H II regions at locations where their number density is high. Thus, most of their emission towards W43 and the $\ell \sim 24^{\circ}$ region must be caused by discrete H II regions rather than the DIG. Overall, the total integrated intensity of our maps is $46 \%$ larger in the $45 \mathrm{~km} \mathrm{~s}^{-1}$ component compared with the Alves et al. (2015) data. This perhaps indicates that we are more sensitive to the diffuse gas.

\subsubsection{The $100 \mathrm{~km} \mathrm{~s}^{-1}$ Gas Component}

While the interpolated $45 \mathrm{~km} \mathrm{~s}^{-1}$ map shows poor agreement with the Alves et al. (2015) data, our $100 \mathrm{~km} \mathrm{~s}^{-1}$ map is strongly correlated with the $1.4 \mathrm{GHz} \mathrm{RRL}$ emission data (Figure 3.6, bottom panel). By-eye comparison of the two maps (Figure 3.4) indicates that we are more sensitive to the diffuse gas component, especially at lower Galactic longitudes. The total integrated intensity of our data is $10 \%$ larger in the $100 \mathrm{~km} \mathrm{~s}^{-1}$ component compared to Alves et al. (2015) (see Table 3.2). Figure 3.5 (top panel) shows that much of the $100 \mathrm{~km} \mathrm{~s}^{-1}$ emission from the DIG may be associated with the Scutum spiral arm. The higher velocities of the DIG compared to the Scutum arm may indicate that we are observing strong streaming motions in this direction (see Bania et al., 2012). Alternatively, the DIG may be located near 

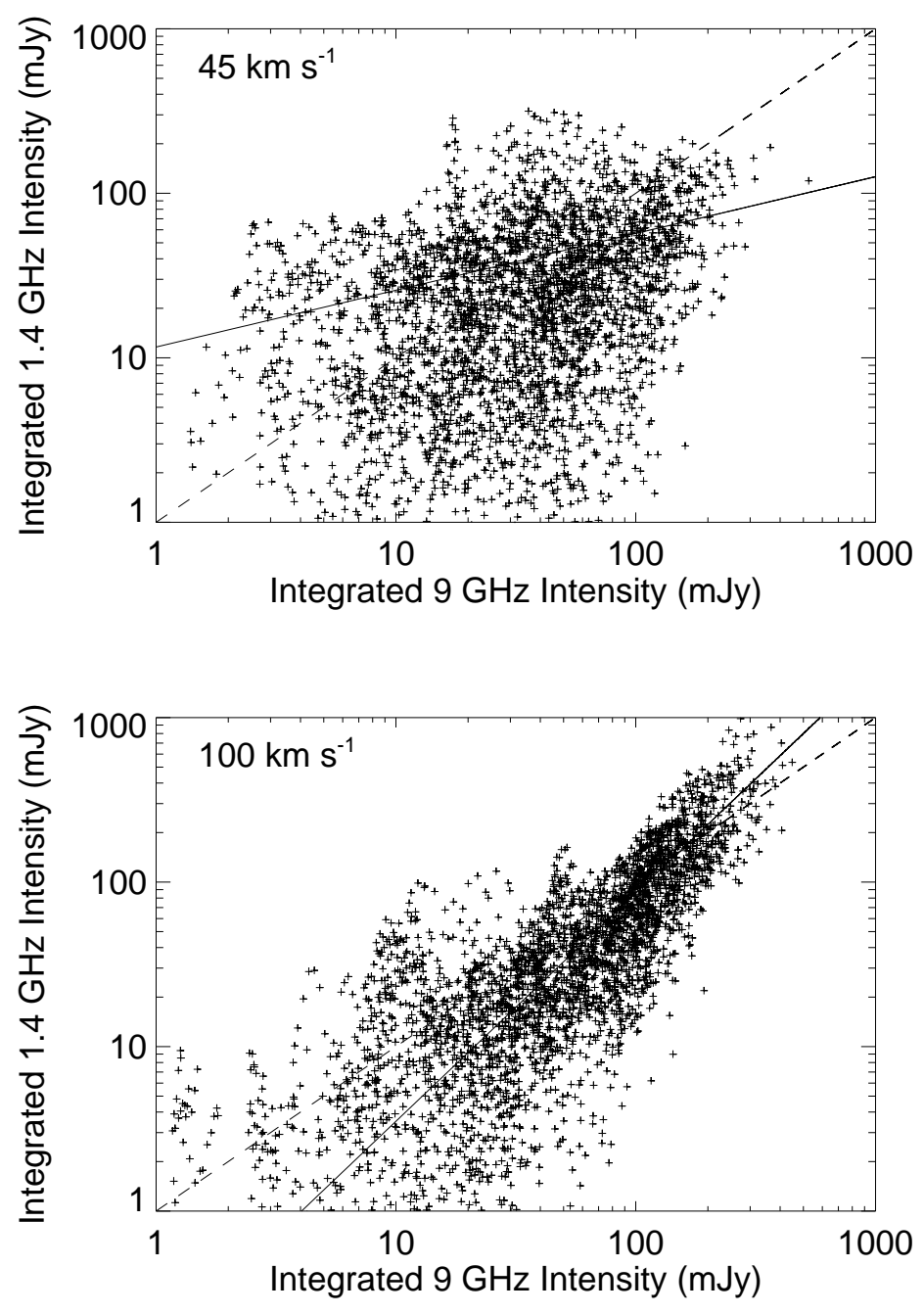

Figure 3.6: Top: Correlation of the diffuse gas emission between our interpolated data at $9 \mathrm{GHz}$ and the $1.4 \mathrm{GHz}$ map from Alves et al. (2015) for the $45 \mathrm{~km} \mathrm{~s}^{-1}$ velocity component. We assume a GBT gain of $2 \mathrm{~K} \mathrm{Jy}^{-1}$ and integrate the intensity over a pixel size of $36 \mathrm{sq}$. arcmin for both data sets. The solid line is a power-law fit of the form $y=a x^{b}$, with $a=11.6321 \pm 0.0295$ and $b=0.3452 \pm 0.0006$. The dashed line is a 1:1 relation. Bottom: Same, for the $100 \mathrm{~km} \mathrm{~s}^{-1}$ velocity component. The power-law fit parameters are $a=0.1440 \pm 0.0003$ and $b=1.3874 \pm 0.0004$. 
the inner edge of the Scutum tangency where it is falling into the arm's gravitational potential, as suggested by Langer et al. (2017).

The directions of strong emission in the two velocity ranges are slightly correlated, such that locations of strong emission from the DIG near $45 \mathrm{~km} \mathrm{~s}^{-1}$ mostly have strong emission near $100 \mathrm{~km} \mathrm{~s}^{-1}$ as well. The correlation is more significant towards the W43 region, whereas it is weak near $\ell \sim 24^{\circ}$ as shown in Figure 3.7. This may suggest that the two velocity ranges towards W43 represent flows of interacting ionized gas (see §3.4.1.3), whereas the two velocity ranges towards the $\ell \sim 24^{\circ}$ region could be caused by DIG emission at two distinct distances.

\subsubsection{H I and the Diffuse Gas}

It is uncertain whether a substantial amount of cold H I gas can coexist with the diffuse ionized gas in regions with strong RRL emission from the DIG. If the radiation field in such regions is strong enough to ionize a large fraction of the gas, we may be able to observe a depletion in H I at locations and velocities of strong DIG emission (Miller \& Cox, 1993; Domgörgen \& Mathis, 1994).

This relationship has been probed by Zurita et al. (2002) who find that the H I distribution does not correlate (or anti-correlate) at all with the diffuse $\mathrm{H} \alpha$ emission tracing the ionized gas in the face-on galaxy NGC 157. The angular resolution of their H I map, however, does not match the better resolution of their $\mathrm{H} \alpha$ map. As a result, they would not be able to resolve H I depletion cavities much smaller than $1 \mathrm{kpc}$. A previous study by Reynolds et al. (1995) analyzed the same correlation for $\mathrm{H} \alpha$-emitting $\mathrm{HI}$ clouds in the Milky Way. They find that the neutral and ionized components in these clouds are likely spatially separated. Since they only observed a relatively small region of the sky away from the Galactic plane, their available sample size is limited.

We use the VGPS H I data to test whether regions with strong emission from the DIG show a deficiency in H I. The VGPS data cubes have a spatial resolution of $1^{\prime} \times 1^{\prime}$ and a spectral resolution of $1.56 \mathrm{~km} \mathrm{~s}^{-1}$. For the $\mathrm{W} 43$ region near $\ell \sim 31^{\circ}$ we

find an apparent $\mathrm{H}$ I depletion cavity at $92 \mathrm{~km} \mathrm{~s}^{-1}$ (Figure 3.8, top left panel) which 

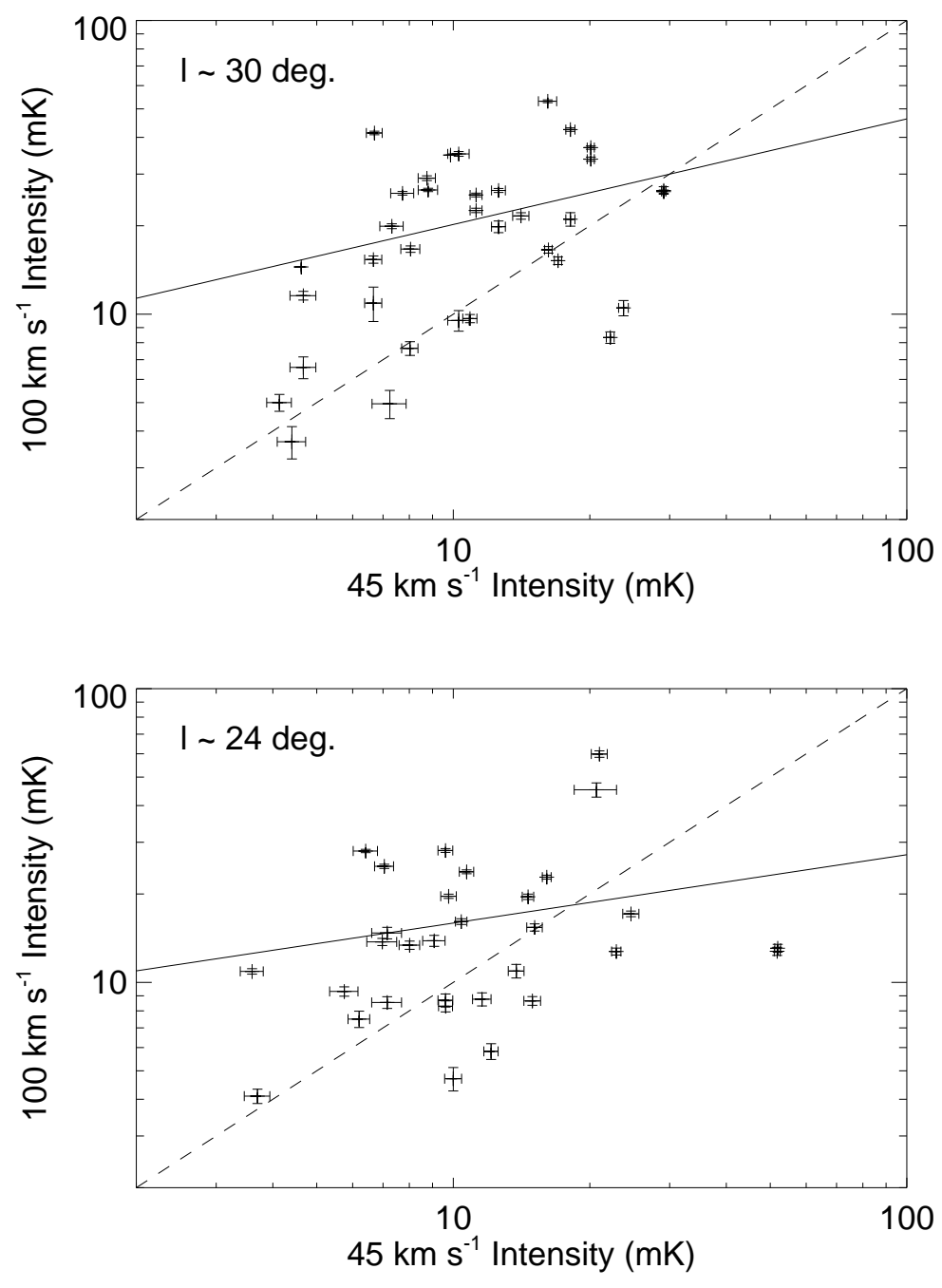

Figure 3.7: Top: Correlation between the observed $45 \mathrm{~km} \mathrm{~s}^{-1}$ and $100 \mathrm{~km} \mathrm{~s}^{-1}$ DIG intensities towards W43 $\left(\ell \sim 30^{\circ}\right)$. Each data point corresponds to a location where the RRL spectrum shows two hydrogen line profiles: one with a LSR velocity in the $25-65 \mathrm{~km} \mathrm{~s}^{-1}$ range (the $45 \mathrm{~km} \mathrm{~s}^{-1}$ component), and one with a LSR velocity in the 80$125 \mathrm{~km} \mathrm{~s}^{-1}$ range (the $100 \mathrm{~km} \mathrm{~s}^{-1}$ component). Error bars are $\pm 1 \sigma$. The solid line is a power-law fit of the form $y=a x^{b}$, with $a=8.8281 \pm 0.3453$ and $b=0.3595 \pm 0.0149$. The $\mathrm{R}^{2}$ of the regression is 0.141 . The dashed line is a 1:1 relation. Bottom: Same, for the $\ell \sim 24^{\circ}$ region. Here, $a=9.3157 \pm 0.4008$ and $b=0.2327 \pm 0.0160\left(\mathrm{R}^{2}=0.058\right)$. 
is consistent with our strong DIG emission shown in Figure 3.4. We find a similar depletion cavity for the $\ell \sim 23^{\circ}$ region at $60 \mathrm{~km} \mathrm{~s}^{-1}$ (see Figure 3.8, top right panel) which is, however, offset by $\sim 1.5^{\circ}$ from the strong DIG emission seen in the $45 \mathrm{~km} \mathrm{~s}^{-1}$ component near $\ell \sim 24.5^{\circ}$ (Figure 3.4). Our large number of RRL pointings near the $\ell \sim 24^{\circ}$ region makes it unlikely that this offset is an artifact from our interpolation algorithm. We did not find any other strong H I depletion cavities in the velocity ranges of significant emission from the DIG for the sky zones in Figure 3.8.

Comparison of our RRL data with ${ }^{13} \mathrm{CO}$ maps casts doubt on whether strong ionized gas emission is usually spatially associated with a deficiency in HI. Using ${ }^{13} \mathrm{CO}$ GRS data, we can determine for both our regions whether these deficiencies are caused by H I self-absorption or due to an actual lack of H I gas. H I self-absorption, first described in detail by Knapp (1974), is usually correlated with CO emission features (Burton et al., 1978; Garwood \& Dickey, 1989). We find substantial ${ }^{13} \mathrm{CO}$ emission at the two directions and velocities (Figure 3.8, bottom panels). In fact, the integrated ${ }^{13} \mathrm{CO}$ emission found at $\ell \sim 31^{\circ}$ and $\ell \sim 23^{\circ}$ is among the strongest within the range of the GRS. This suggests that the lack in H I emission is caused by H I self-absorption and is not due to an actual deficiency in H I gas.

\subsubsection{Correlation with $8.0 \mu \mathrm{m}$ Intensity}

Emission from polycyclic aromatic hydrocarbons (PAHs) within the $8.0 \mu \mathrm{m}$ band is usually caused by softer ultra-violet (UV) radiation than that responsible for RRL emission from H II regions. For example, Robitaille et al. (2012) show, using radiative transfer models, that most of $\mathrm{PAH}$ heating is provided by $\mathrm{B}$ stars, compared to RRL emission typically caused by $\mathrm{O}$ stars. While the $8.0 \mu \mathrm{m}$ emission is often associated with strong PDRs surrounding discrete H II regions, there exists significant $\mathrm{PAH}$ emission that originates from the diffuse gas without nearby $\mathrm{H}$ II regions. Below, we analyze this "diffuse" PAH emission and its relation to the DIG. In the bottom panel of Figure 3.9 we show a map of the point-source subtracted $8.0 \mu \mathrm{m}$ Spitzer GLIMPSE emission (Benjamin et al., 2003; Churchwell et al., 2009). Since there is also strong $8.0 \mu \mathrm{m}$ emission from discrete H II regions, we blank out these regions 

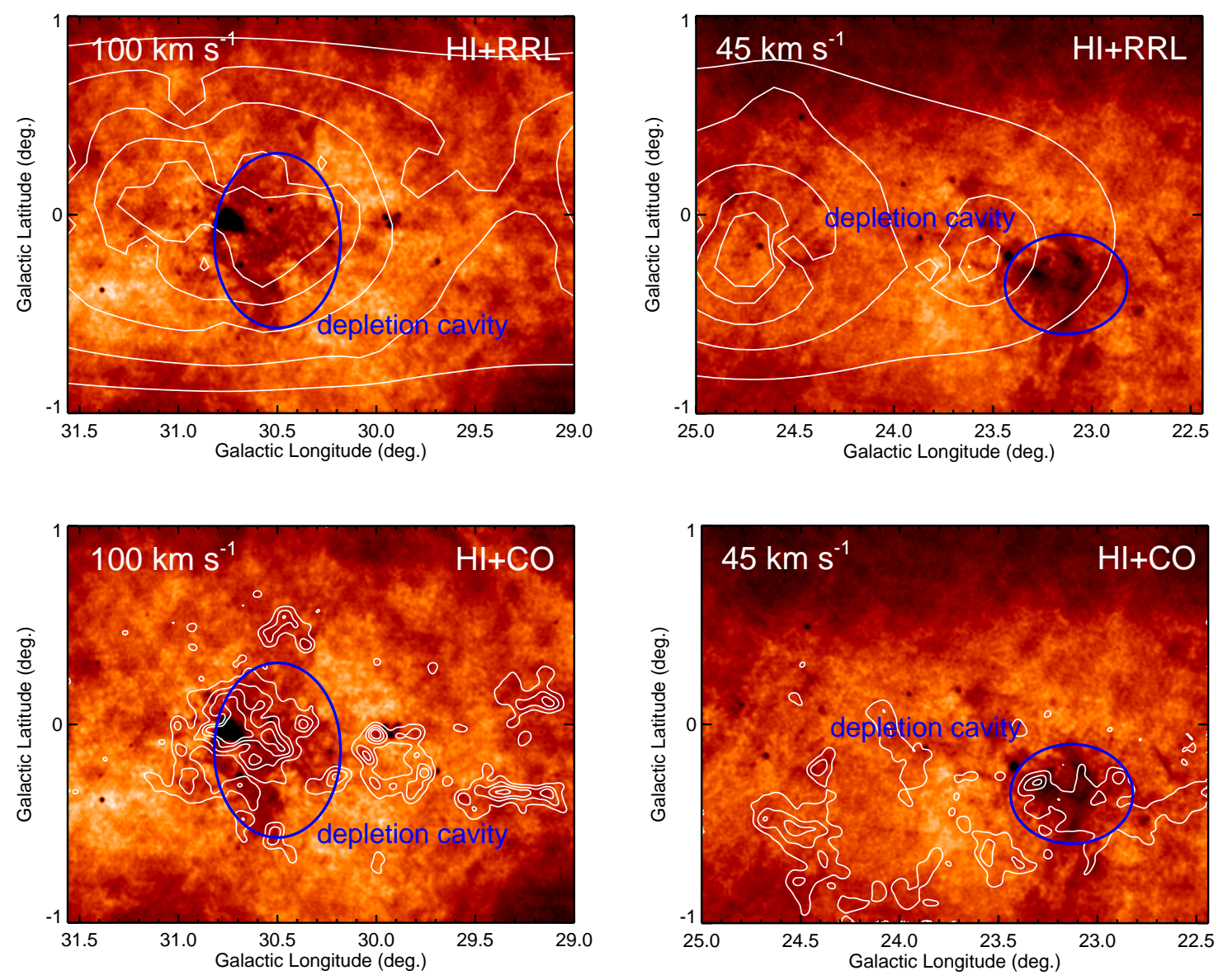

Figure 3.8: Comparison of RRL emission from the DIG with H I and CO emission reveals that deficiencies in $\mathrm{HI}$ emission are more likely due to self-absorption rather than gas displacement by the DIG. Top left: The VGPS H I map at $92 \mathrm{~km} \mathrm{~s}^{-1}$ for the $29^{\circ}<\ell<31.5^{\circ}$ region, linearly scaled from 0 to $150 \mathrm{~K}$ brightness temperature. The white contours show our interpolated diffuse gas map at the $100 \mathrm{~km} \mathrm{~s}^{-1}$ velocity component at $4,8,12,16$, and $20 \mathrm{mK}$. A deficiency in $\mathrm{H} \mathrm{I}$ emission is clearly visible at $\ell \sim 30.5^{\circ}$, the location of W43. Top right: VGPS H I map at $60 \mathrm{~km} \mathrm{~s}^{-1}$ for the $22.5^{\circ}<\ell<25^{\circ}$ region, with contours of our $45 \mathrm{~km} \mathrm{~s}^{-1}$ diffuse gas map and same increments as above. An H I deficiency is visible at $\ell \sim 23^{\circ}$ which is, however, not spatially correlated with the diffuse gas emission. Bottom left: Same as top left, but with blue GRS ${ }^{13} \mathrm{CO}$ contours at the same velocity. Contours are at $0.5,1,1.5$, and $2 \mathrm{~K}$ antenna temperature. The $\mathrm{CO}$ emission is spatially correlated with the $\mathrm{HI}$ bubble above, indicating that the deficiency in $\mathrm{HI}$ emission may be caused by $\mathrm{HI}$ self-absorption. Bottom right: Same, for the $22.5^{\circ}<\ell<25^{\circ}$ region at $60 \mathrm{~km} \mathrm{~s}^{-1}$. 


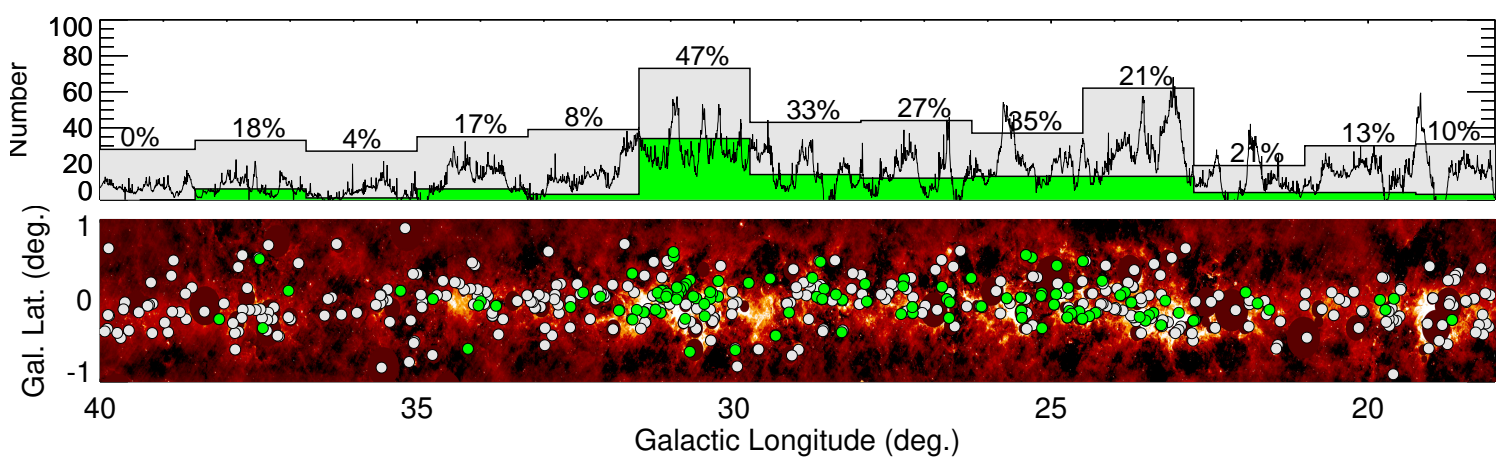

Figure 3.9: Locations of multiple-velocity H II regions. In the bottom panel, the background image is the point-source subtracted $8.0 \mu \mathrm{m}$ Spitzer GLIMPSE emission, on a square-root scale ranging from -5 to $50 \mathrm{MJy} \mathrm{sr}^{-1}$. Multiple-velocity H II regions are shown in green and single velocity H II regions are shown in gray. Emission around H II regions is blanked out according to their positions and sizes from the WISE catalog (Anderson et al., 2014). In the top panel we show histograms of the number of single-velocity H II regions (gray) and multiple-velocity H II regions (green). The numbers above the histogram indicate the percentage of multiple-velocity regions over the given longitude range. The black curve in the top panel shows the $8.0 \mu \mathrm{m}$ GLIMPSE emission from the bottom panel integrated over all latitudes. There is a correlation between the number of H II regions and the $8.0 \mu \mathrm{m}$ intensity (see Figure 3.10).

based on their corresponding positions and sizes from the WISE catalog, Version 1.4 (Anderson et al., 2014). In the upper panel of Figure 3.9 we show histograms of the location of single-velocity and multiple-velocity H II regions.

We observe a correlation between the location of discrete $\mathrm{H}$ II regions and the intensity of the $8.0 \mu \mathrm{m}$ emission (see Figure 3.10). Both of these diffuse emission components should be caused by UV photons leaking from the discrete H II regions. The PAHs responsible for the $8.0 \mu \mathrm{m}$ emission are destroyed in the hard UV radiation within an H II region (Voit, 1992; Povich et al., 2007), but can survive where the radiation field is softer, i.e., in the diffuse ISM. The correlation therefore suggests that either a significant amount of the (soft) UV photons responsible for the $8 \mu \mathrm{m}$ emission is leaking from the discrete HII regions or that the harder UV radiation produced by the $\mathrm{O}$ stars softens as it escapes into the ISM. Such a radiation softening has been suggested by Reynolds \& Tufte (1995) and was recently observed indirectly for the compact H II region NGC 7538 (Luisi et al., 2016).

We also observe a correlation between the hard UV radiation field within the 


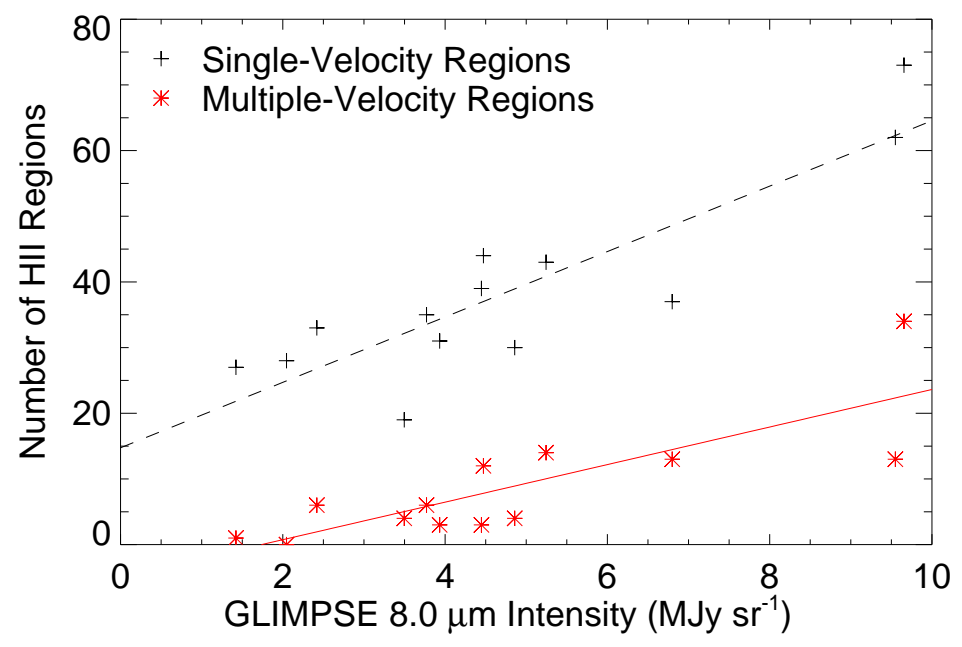

Figure 3.10: Correlation between $8.0 \mu \mathrm{m}$ emission from PAHs and the number of H II regions. Each data point represents one histogram bin from Figure 3.9, averaged over the entire $|b|<1^{\circ}$ latitude range. The dashed line is a linear fit of the form $y=a+b x$ for the single-velocity regions, with $a=14.743 \pm 0.6056$ and $b=4.9803 \pm 0.1127$. The solid line is the fit for the multiple-velocity regions, with $a=-4.9657 \pm 0.6057$ and $b=2.8588 \pm 0.1127$. The correlation indicates that a significant amount of soft UV radiation is present near discrete $\mathrm{H}$ II regions.

DIG and the softer UV radiation field responsible for PAH emission. Our method probes the radiation field strengths by using the observed RRL emission from the DIG and the $8.0 \mu \mathrm{m}$ emission intensities as a proxy for the diffuse hard UV and soft UV radiation field strengths, respectively. A correlation between the intensities of these two emission components indirectly tests for a correlation between the radiation fields. We determine the diffuse $8.0 \mu \mathrm{m}$ background by integrating the $8.0 \mu \mathrm{m}$ flux in a circular $41^{\prime \prime}$ aperture centered at each of the 135 off-target directions. By only using the off-target directions, we ensure that we are only sampling the diffuse $8.0 \mu \mathrm{m}$ background, and not the emission associated with H II region PDRs. We then compute the fluxes using our Kang software ${ }^{3}$. Kang is an astronomical visualization and analysis package written in IDL. Its relevant functionality here is that it can compute aperture photometry measurements using arbitrary aperture shapes.

We compare the diffuse $8.0 \mu \mathrm{m}$ emission with the integrated intensity from all hydrogen RRLs at all velocities detected at an off-target direction. We plot in Fig-

\footnotetext{
${ }^{3}$ http://www.bu.edu/iar/files/script-files/research/kang/
} 


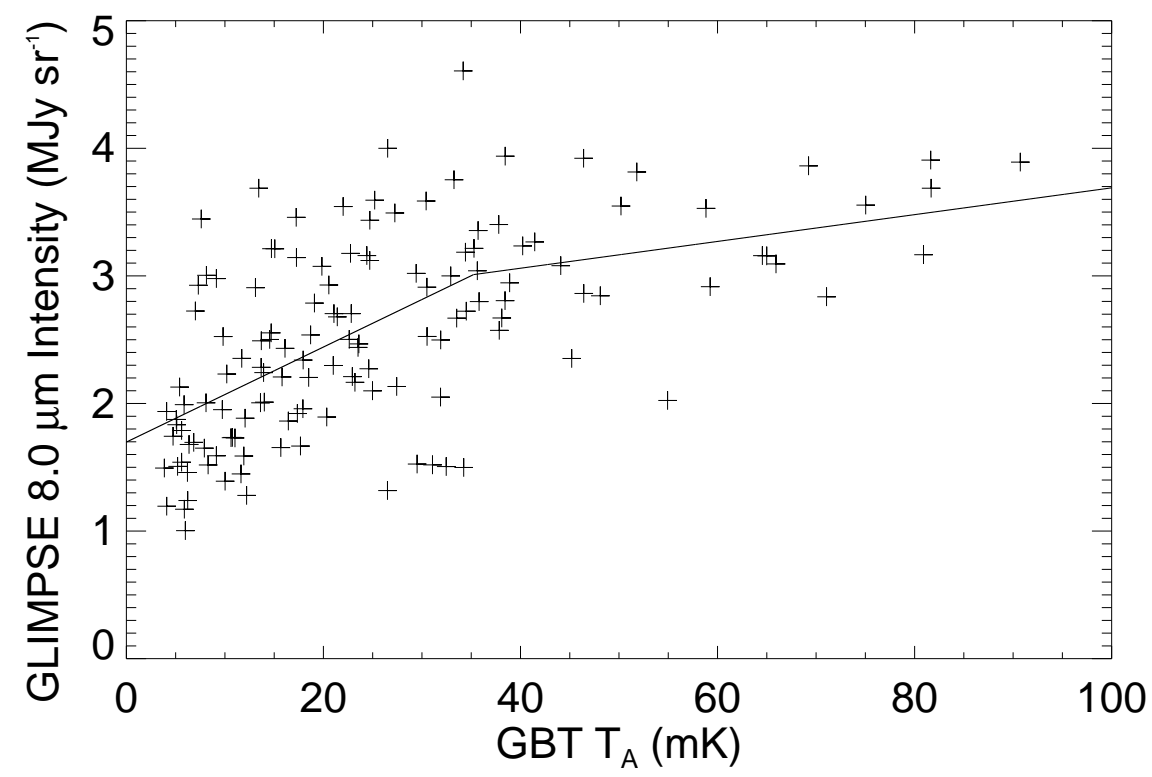

Figure 3.11: Correlation between $8.0 \mu \mathrm{m}$ emission from PAHs and RRL emission from the DIG. The RRL emission is from our pointed GBT observations of the diffuse gas. We average the point-source subtracted $8.0 \mu \mathrm{m}$ Spitzer GLIMPSE intensity over a 41" radius aperture (the beam size of the RRL observations) at the directions of our GBT observations. The two straight lines show linear fits, and indicate that the intensity of the far-UV radiation responsible for the RRL emission is correlated with the softer UV radiation responsible for the $8.0 \mu \mathrm{m}$ emission. The break between the fits is at GBT $T_{A}=35 \mathrm{mK}$ which suggests that above this temperature PAHs are destroyed in highly energetic radiation fields, leading to a saturation in IR emission.

ure 3.11 the correlation between the diffuse $8.0 \mu \mathrm{m}$ emission and the integrated RRL intensity. Figure 3.11 indicates that the hard UV radiation is correlated with the softer UV radiation, as one would expect, although there is quite a large scatter.

Furthermore, the correlation appears to change above $35 \mathrm{mK}$. This may be due to the destruction of the PAH molecules that are largely responsible for the diffuse $8.0 \mu \mathrm{m}$ emission. A similar effect has been observed by Lebouteiller et al. (2011) in the Galactic HII region NGC 3603. Direct PAH destruction typically requires photons with energies $>20 \mathrm{eV}$ which exist in sufficient numbers only in the most energetic radiation fields (e.g., within H II regions). The binding energy of H atoms to PAHs, however, is only $\sim 4.8 \mathrm{eV}$ (Voit, 1992). Thus, even less energetic radiation fields can contribute to PAH dissociation. The relatively strong hydrogen ionizing 
radiation field $(\geq 13.6 \mathrm{eV})$ within regions with substantial $\mathrm{RRL}$ emission from the DIG must therefore partly be responsible for PAH dissociation. Considering that this effect limits the abundance of PAHs in these regions, it comes as no surprise that we observe a saturation in PAH emission.

Clearly, further study of this correlation is required. Most helpful would be additional pointings toward cleaner sight lines where the source of the UV photons can be more easily be determined. A more direct measurement of the radiation field strength is difficult. Recently, Stock \& Peeters (2017) described a technique to estimate the UV field intensity using the ratio between two PAH spectral components at $7.6 \mu \mathrm{m}$ and $7.8 \mu \mathrm{m}$. While this technique has not yet been applied to the diffuse ISM, it may prove useful in constraining properties of the radiation field outside H II regions.

\subsection{Summary}

Here, we analyze the DIG using hydrogen RRL emission line spectra in the range $\ell=18^{\circ}$ to $40^{\circ}$ which are either devoid of $\mathrm{H}$ II region emission or have multiple velocity components. Our data set is comprised of 353 RRL emission line components from the DIG. These allow us to determine the intensity and distribution of the diffuse gas.

We find that the DIG is spatially concentrated in two areas near $\ell=31^{\circ}$ and $\ell=24^{\circ}$, with two dominant velocity components $\left(45 \mathrm{~km} \mathrm{~s}^{-1}\right.$ and $\left.100 \mathrm{~km} \mathrm{~s}^{-1}\right)$ in each of the areas. We investigate the KDA for the two velocity ranges and conclude that the $100 \mathrm{~km} \mathrm{~s}^{-1}$ component has a Galactocentric distance of $\sim 6 \mathrm{kpc}$, corresponding to the location of W43. This suggests that much of the $100 \mathrm{~km} \mathrm{~s}^{-1}$ gas is associated with W43. The origin of the $45 \mathrm{~km} \mathrm{~s}^{-1}$ component is less clear. The intensity of the emission in the two velocity ranges is slightly correlated near $\ell=31^{\circ}$, which may imply that both velocity components originate at a single distance. In this case, the $45 \mathrm{~km} \mathrm{~s}^{-1}$ component may arise from complex streaming motions near the end of the Galactic bar. For the $\ell=24^{\circ}$ region, however, it is unlikely that the observed velocity 
components are due to this effect. As an alternative, we suggest that the $45 \mathrm{~km} \mathrm{~s}^{-1}$ emission may have its origin at a Galactocentric distance of $\sim 12 \mathrm{kpc}$, or a combination of both. Unfortunately, our current data are insufficient to clearly distinguish between these cases, a problem which may be investigated in future work. Future work may also explore in more detail the connection between the DIG observed in RRL emission, the more diffuse component observed in $\mathrm{H} \alpha$, and the different environments these data are tracing.

Since regions with strong RRL emission from the DIG may show a deficiency in H I, we examine data from the VGPS for H I depletion cavities. We find such a bubble in $\mathrm{HI}$ emission for the W43 region at $\ell \sim 31^{\circ}$ at $92 \mathrm{~km} \mathrm{~s}^{-1}$. We also find a second bubble at $\ell \sim 23^{\circ}$ and $60 \mathrm{~km} \mathrm{~s}^{-1}$ which is, however, offset by $\sim 1.5^{\circ}$ from the direction of strong DIG emission at $\ell \sim 24.5^{\circ}$. There is strong ${ }^{13} \mathrm{CO}$ emission associated with these locations, suggesting that the deficiency in H I emission is rather caused by H I self-absorption than an actual lack of H I gas.

The intensity of the RRL emission from the DIG is also correlated with the intensity of diffuse Spitzer GLIMPSE $8.0 \mu \mathrm{m}$ emission, implying that the soft UV photons responsible for creating the infrared emission have a similar origin as the harder UV photons required for the RRL emission. The diffuse $8.0 \mu \mathrm{m}$ emission appears to saturate at locations with the strongest RRL emission suggesting that the PAHs responsible for the $8.0 \mu \mathrm{m}$ emission are destroyed by the radiation field in these regions. 


\section{Chapter 4}

\section{Ionization Profiles of Galactic H II Regions}

\subsection{Abstract}

Using Green Bank Telescope radio recombination line (RRL) data, we analyze the role of leaking radiation from H II regions in maintaining the ionization of the interstellar medium. We observed a sample of eight Galactic H II regions of various sizes, morphologies, and luminosities. For each region the hydrogen RRL intensity decreases roughly as a power-law with distance from the center of the region. This suggests that radiation leaking from the HII region is responsible for the majority of surrounding ionized gas producing RRL emission. Our results further indicate that the hydrogen RRL intensity appears to be fundamentally related to the HII region sizes traced by their photodissociation regions, such that physically smaller H II regions show a steeper decrease in intensity with increasing distance from the region centers. As a result, giant H II regions may have a much larger effect in maintaining the ionization of the interstellar medium. For six of the eight observed H II regions we find a decrease in the ${ }^{4} \mathrm{He}^{+} / \mathrm{H}^{+}$abundance ratio with increasing distance, indicating that He-ionizing photons are being absorbed within the ionization front of the H II region. There is enhanced carbon RRL emission toward directions with strong continuum background, suggesting that the carbon emission is amplified by stimulated emission.

\subsection{Introduction}

H II regions, first described by Strömgren (1939), are regions of ionized gas surrounding $\mathrm{O}$ and B-type stars. Only these short-lived and massive stars emit a sufficient number of high-energy photons $(>13.6 \mathrm{eV})$ to fully ionize their surroundings. H II regions are among the most luminous objects in our Galaxy at radio wavelengths

The research contained in this chapter was published as Luisi et al. (2019), ApJS, 241, 2.

Contributing authors: L. D. Anderson, B. Liu, D. A. Roshi, E. Churchwell. 
and can be studied using radio recombination line (RRL) and radio free-free continuum emission. Compared to observations at near- to mid-infrared (IR) wavelengths, these radio observations are faint, but have the benefit of being essentially free from extinction.

Between the H II region and the ambient diffuse interstellar medium (ISM) lies the $\mathrm{HI}$ front, the boundary between fully ionized hydrogen within the region and neutral hydrogen outside, followed by a photodissociation region (PDR). In PDRs, hydrogen is predominantly neutral, but carbon and other species with ionization potentials lower than that of hydrogen are mostly ionized. PDRs can be studied using numerous atomic or molecular transitions, e.g., IR emission from polycyclic aromatic hydrocarbons (PAHs) or $\mathrm{C}^{+}$emission at $158 \mu \mathrm{m}$.

Low-density diffuse gas known as the "Diffuse Ionized Gas" or "Warm Ionized Medium" (WIM) is a major component of the ISM of our Galaxy. The WIM, with electron temperatures ranging from $6000 \mathrm{~K}$ to $10,000 \mathrm{~K}$, accounts for over $90 \%$ of all ionized hydrogen in the ISM (Haffner et al., 2009). It has a scale height of $\sim 1 \mathrm{kpc}$ and was found to be in a lower ionization state than gas in H II regions (Haffner et al., 1999; Madsen et al., 2006). The "Extended Low-Density Medium" (ELDM), another diffuse component of the ISM (see Gottesman \& Gordon, 1970; Mezger, 1978), has a smaller scale height of only $\sim 100$ pc and has been observed to be spatially correlated with the locations of discrete H II regions (Alves et al., 2012).

While it is still not fully known how the WIM maintains its ionization (Haffner et al., 2009), it is believed that O-stars are the most likely source of ionizing photons since all other possible ionization mechanisms (e.g., supernova explosions) cannot fulfill the energy requirements (Domgörgen \& Mathis, 1994; Hoopes \& Walterbos, 2003). Given the distribution of the WIM, however, it remains unclear precisely how the radiation from O-stars within H II regions is able to propagate through their surrounding PDRs and across kiloparsec size-scales into the ISM. While Wood et al. (2010) argue that a supernova-driven turbulent ISM has low-density paths that would allow ionizing photons to reach and ionize gas several kiloparsecs above the midplane, it is unknown whether this scenario could explain the WIM distribution in the Galactic 
plane.

Observations have shown that a significant amount of ionizing radiation is leaking from individual H II regions. While most of these analyses focus on H II regions in external galaxies (e.g., Oey \& Kennicutt, 1997; Zurita et al., 2002; Giammanco et al., 2005; Pellegrini et al., 2012), a few studies were performed on Milky Way H II regions. Anderson et al. (2015) showed using H-alpha emission data that the Galactic H II region RCW 120 is leaking 25\% of its ionizing radiation into the ISM. They further showed that the PDR is clumpy at $8.0 \mu \mathrm{m}$ and that photons preferentially escape through low-density pathways into the ISM. We performed a similar analysis on the compact Galactic H II region NCG 7538 (Luisi et al., 2016, hereafter L16) using Green Bank Telescope (GBT) RRL and radio continuum data to better understand how a single H II region may contribute to the ionization of the WIM. We computed an ionizing leaking fraction of $15 \pm 5 \%$ and found that, unlike giant H II region complexes, the radiation leaking from NCG 7538 seems to only affect the local ambient medium.

It is not well-understood how emission from the WIM is affected by the presence of H II regions, their sizes, and morphologies. We showed in L16 that the hydrogen RRL intensity around the compact H II region NGC 7538 decreases rapidly with distance from the central region, whereas the RRL emission decrease in the giant H II region complex W43 is much less steep. This result implies that giant H II regions may have a much larger effect on maintaining the ionization of the WIM compared to compact H II regions. In fact, Zurita et al. (2002) suggest that essentially all ionizing radiation escapes from H II regions with the highest luminosities. Using a model from Beckman et al. (2000), Zurita et al. (2000) suggest that radiation leaking from luminous clusters of HII regions may be sufficient to ionize the diffuse gas. As a result, a large escape fraction of less luminous regions may not necessarily be required to maintain the ionization of the WIM.

The spectrum of the radiation field provides additional information on the physical processes within HII regions and the effect of leaking radiation on the WIM. Within an H II region, the radiation field depends on the temperature of the ionizing 
star(s). The ratio of emitted helium $(E>24.6 \mathrm{eV})$ to hydrogen $(E>13.6 \mathrm{eV})$ ionizing photons, $Q_{1} / Q_{0}$, can be estimated indirectly by measuring the $N\left({ }^{4} \mathrm{He}^{+}\right) / N\left(\mathrm{H}^{+}\right)$ ionic abundance ratio. While $Q_{1} / Q_{0}$ is determined by the temperature of the ionizing star(s), its value is only $\sim 0.25$ even for the hottest O3V stars (see Martins et al., 2005; Draine, 2011). For an O9V star, $Q_{1} / Q_{0}$ is reduced to 0.015. As the radiation travels through the stellar atmosphere, metals will selectively absorb more energetic photons in a process known as line blanketing. While dependent on the metallicity of the star, this process will generally result in a decrease in the ratio of He-to-H ionizing photons (e.g., Pankonin et al., 1980; Afflerbach et al., 1997). The He-to-H ionizing photon ratio is also affected by dust, which causes selective attenuation of ultraviolet (UV) photons.

As radiation propagates through an $\mathrm{H}$ II region, its spectrum changes further due to absorption and re-emission processes. Wood \& Mathis (2004) suggested that these interaction processes preferentially result in a hardening of the $\mathrm{H}$-ionizing continuum and a suppression of He-ionizing photons. Recently, Weber et al. (2019) showed that for O-stars with low effective temperatures $(<35000 \mathrm{~K})$ nearly the entire He-ionizing radiation is absorbed within the HII regions. The radiation hardening has been demonstrated by Osterbrock (1989) based on the dependence of the absorption crosssection on frequency: ionizing photons with energies $\mathrm{E} \gtrsim 13.6 \mathrm{eV}$ are preferentially absorbed by hydrogen compared to photons with much higher energies. Since the ionization cross-section of $\mathrm{He}$ is much greater than that of $\mathrm{H}$, a large fraction of He-ionizing photons is absorbed well within the ionization front of the $\mathrm{HII}$ region, resulting in a depletion of He-ionizing photons outside the PDR.

The suppression of He-ionizing photons implies a reduced $N\left({ }^{4} \mathrm{He}^{+}\right) / N\left(\mathrm{H}^{+}\right)$ionic abundance ratio by number outside the $\mathrm{H}$ II region as there will be a fewer number of photons with sufficient energy to ionize He compared to H. Such H II regions may be density bounded beyond the $\mathrm{He}^{+}$zone, but within the $\mathrm{H}^{+}$zone (see Reynolds et al., 1995). This effect was observed by Pankonin et al. (1980), who show that the ionized helium abundance in the Orion Nebula decreases with distance from the exciting star. We also indirectly confirmed these results in L16 for the compact H II 
region NCG 7538 by observing a decrease in the $N\left({ }^{4} \mathrm{He}^{+}\right) / N\left(\mathrm{H}^{+}\right)$ratio with increasing distance from the region's central position. It is, however, unknown whether these findings are applicable to Galactic H II regions in general and their relation to age or geometry of the region.

Previous observational work and simulations confirm that the ratio of He-to-H ionizing photons in the WIM is lower than that found in H II regions. In a study of optical emission lines toward faint $\mathrm{H} \alpha$-emitting regions in the Milky Way, Madsen et al. (2006) show that the $\mathrm{HeI} / \mathrm{H} \alpha$ line ratio is suppressed compared to that of H II regions, indicating a softer radiation field. Using Monte Carlo photoionization simulations, Wood \& Mathis (2004) suggest that this line ratio depends strongly on the $\mathrm{HII}$ region leaking fraction. They find that $\mathrm{HeI} / \mathrm{H} \alpha$ is significantly reduced only for low escape fractions $(\sim 15 \%)$. This result is in disagreement with Roshi et al. (2012), who observed a $N\left({ }^{4} \mathrm{He}^{+}\right) / N\left(\mathrm{H}^{+}\right)$upper limit of only 0.024 in the diffuse gas near the HII region G49, despite an apparent escape fraction of $\sim 63 \%$ and $N\left({ }^{4} \mathrm{He}^{+}\right) / N\left(\mathrm{H}^{+}\right)$ratios of $>0.066$ within the $\mathrm{H}$ II region (Churchwell et al., 1974; Lichten et al., 1979; Thum et al., 1980; McGee \& Newton, 1981; Mehringer, 1994; Bell et al., 2011). Clearly, a larger sample size is required for further study.

The goal of this study is to observe a variety of $\mathrm{H}$ II regions to determine the role of leaking radiation from H II regions in maintaining the ionization of the WIM. Our observed sample includes H II regions of different sizes and morphologies for which the PDR boundary can be identified and for which the spectral class of its central star(s) is known. The properties of our observed H II regions are summarized in Table 4.1, which lists the source name, the Galactic longitude and latitude, the radius of the H II region, its Galactocentric radius, the distance to the Sun, and the spectral type of the ionizing source(s).

The GBT RRL observations are described in $\S 4.3$ of this dissertation and we outline the process of defining PDR boundaries in $\S 4.4$. In $\S 4.5$ and $\S 4.6$ we analyze the hydrogen RRL emission around the observed $\mathrm{H}$ II regions and derive $N\left({ }^{4} \mathrm{He}^{+}\right) / N\left(\mathrm{H}^{+}\right)$ ionic abundance ratios for the observed directions, respectively. Physical properties of the ionized gas, including electron temperatures, emission measures, and electron 
densities are derived in $\S 4.7$. In $\S 4.8$ a line profile analysis is performed as an alternative method to derive electron temperatures. We analyze carbon and doubly-ionized helium emission in $\S 4.9$ and $\S 4.10$, respectively, and conclude in $\S 4.11$.

Table 4.1: H II Region Properties

\begin{tabular}{|c|c|c|c|c|c|c|}
\hline Source & $\begin{array}{c}\ell \\
\text { (deg.) }\end{array}$ & $\begin{array}{c}b \\
\text { (deg.) }\end{array}$ & $\begin{array}{l}\text { Radius } \\
\text { (pc) }\end{array}$ & $\begin{array}{l}R_{\text {gal }} \\
(\mathrm{kpc})\end{array}$ & $\begin{array}{c}\text { Distance } \\
(\mathrm{kpc})\end{array}$ & Spectral Type \\
\hline M17 (S45) & 15.098 & -0.729 & 11.3 & 6.6 & 2.0 & $\mathrm{O} 4-\mathrm{O} 4^{[1]}$ \\
\hline M16 (S49) & 16.993 & 0.874 & 14.2 & 6.1 & 2.6 & $\mathrm{O} 5^{[2]}$ \\
\hline $\mathrm{N} 49$ & 28.823 & -0.226 & 3.6 & 4.5 & 5.5 & $\mathrm{O} 5^{[3]}$ \\
\hline $\mathrm{G} 45.45+0.06(\mathrm{G} 45)$ & 45.453 & 0.055 & 9.9 & 6.5 & 8.4 & $\mathrm{O} 6^{[4]}$ \\
\hline S104 & 74.769 & 0.622 & 2.4 & 8.2 & 1.5 & $\mathrm{O} 5^{[5]}$ \\
\hline S206 & 150.596 & -0.955 & 7.9 & 11.6 & 3.4 & $\mathrm{O} 6^{[6]}$ \\
\hline Orion (S281) & 209.107 & -19.509 & 3.4 & 8.9 & 0.4 & $\mathrm{O} 6^{[7]}$ \\
\hline G29.96-0.02 (G29) & 29.956 & -0.020 & 2.4 & 4.7 & 5.3 & $\mathrm{O} 5.5^{[8]}$ \\
\hline
\end{tabular}

References - [1] Broos et al. (2007), [2] Sota et al. (2011), [3] Watson et al. (2008), [4] Moisés et al. (2011), [5] Lahulla (1985), [6] Georgelin et al. (1973),

[7] O'Dell et al. (2017), [8] Cesaroni et al. (1994).

\subsection{Observations and Data Reduction}

We observed 8 H II regions with the Green Bank Observatory GBT from 2017 February to 2017 May. The properties of the observed HII regions are given in Table 4.1. For each targeted HII region, several positions within and outside the region's PDR were observed (see $§ 4.4$ for a description of how the PDR boundaries were determined). We employed total-power position-switching observations with variable off-source and on-source integration times, ranging from 3 to 36 minutes, depending on the expected brightness of the position. For integration times exceeding 6 minutes, the observation was split up into blocks of 6 minutes each to reduce the overall impact of radio-frequency interference (RFI).

The individual pointings for each observed H II region lie along an imaginary line on the plane of the sky intersecting the region center. The angle of the line was chosen such that there is as little confusion by other radio sources (e.g., nearby HII 
regions) as possible. For each targeted H II region, between 7 and 17 positions were observed. The goal was to include as many positions as possible within the regions' PDR boundaries, separated by as little as the average GBT half-power beam width (HPBW) of $123^{\prime \prime}$. Outside the PDR boundaries, our pointings are spaced apart by one to four GBT HPBWs, depending on the spatial extent of the source and our total available observing time. For the largest regions, we sample up to a maximum distance of $47^{\prime}$ from the center. The locations of the observed positions for each source are shown in Figure 4.1. The locations are labeled according to their predominant direction from the region center in Galactic coordinates ( $\mathrm{N}$ denotes north, $\mathrm{S}$ denotes south, E denotes east, and $\mathrm{W}$ denotes west) and their distance from the center of the regions in multiples of $2 \times \mathrm{HPBW}$. The coordinates of all observed directions are given in Table A.1, which lists the source, the source coordinates, and the location of the observed direction with respect to the PDR boundary (see $§ 4.4)$.

The GBT C-band instantaneous bandpass in combination with the VEGAS backend includes $22 \mathrm{Hn} \alpha$ lines, $19 \mathrm{Hn} \beta$ lines, and $8 \mathrm{Hn} \gamma$ lines between 4.054 and $7.793 \mathrm{GHz}$. In our setup, the $\alpha$ lines range from $n=95$ to $n=117$, the $\beta$ lines range from $n=120$ to $n=146$, and the $\gamma$ lines range from $n=138$ to $n=147$ (see Luisi et al., 2018). In addition, we tuned to $7 \operatorname{HeIn} \alpha$ lines and 8 molecular lines, including formaldehyde and methanol. To achieve a higher signal-to-noise ratio, we average together all $\operatorname{Hn} \alpha, \operatorname{Hn} \beta$, and $\operatorname{Hn} \gamma$ lines, respectively, using $\mathrm{TMBIDL}^{1}$ after first regridding and shifting the spectra so that they are aligned in velocity (see Balser, 2006). Spectra affected by RFI were discarded before averaging. The averaged spectra were smoothed to a resolution of $1.86 \mathrm{~km} \mathrm{~s}^{-1}$ and a fourth-order polynomial baseline was subtracted.

Gaussian models were fit to the $\mathrm{H}$ and He profiles for which the signal-to-noise ratio $(\mathrm{S} / \mathrm{N})$ is at least 5 as defined by the method given by Lenz \& Ayres (1992),

$$
\mathrm{S} / \mathrm{N}=0.7\left(\frac{T_{\mathrm{L}}}{\mathrm{rms}}\right)\left(\frac{\Delta V}{\Delta V_{\mathrm{k}}}\right)^{0.5}
$$

\footnotetext{
${ }^{1}$ V7.1, see https://github.com/tvwenger/tmbidl.git.
} 
where $T_{\mathrm{L}}$ is the peak line intensity, rms is the root-mean-squared spectral noise, $\Delta V$ is the full width at half maximum (FWHM) of the line, and $\Delta V_{\mathrm{k}}=1.86 \mathrm{~km} \mathrm{~s}^{-1}$ is the FWHM of the Gaussian smoothing kernel. We also fit carbon lines with a $\mathrm{S} / \mathrm{N}$ of at least 3. Here, the narrow width of the carbon lines allows for a lower $\mathrm{S} / \mathrm{N}$ threshold as they are less likely to be confused with baseline fluctuations. In the few cases where more than one hydrogen line velocity component is detected, we assume that the brightest component is due to the H II region.

The continuum antenna temperature $T_{\mathrm{C}}$ was derived for all observed positions from the continuum background of our averaged spectral line data by removing all lines above the $3 \sigma$ level. We also remove $20 \%$ of all channels towards each end of the bandpass, since the bandpass edges are prone to instabilities, and average the antenna temperature over the remaining baseline to find $T_{\mathrm{C}}$. Since the baseline stability is the dominant uncertainty contribution, the error in $T_{\mathrm{C}}$ is estimated by averaging over individual segments of each baseline, 1000 channels in width, and by computing the standard deviation between these segments. All continuum antenna temperatures are given in Table A.3, which lists the source, $T_{\mathrm{C}}$, the LTE electron temperatures $\left(T_{\mathrm{e}}^{*}\right.$; see $\S 4.7 .1$ ), and the emission measures (EM; see $§ 4.7 .2)$, including all $1 \sigma$ uncertainties.

The weather conditions were good for most, but not all, observing sessions. Skies were cloudy for $\sim 25 \%$ of our observations, with intermittent rain and snow. While the effect of weather on our RRL observations is small, the derived continuum temperatures are more strongly affected by cloudy skies. We assume that our uncertainties for $T_{\mathrm{C}}$ shown above include the impact of changing weather conditions.

The averaged Hn $\alpha$ spectra are shown in Figure A.1 and the derived $\operatorname{Hn} \alpha, \operatorname{Hn} \beta$, and Hn $\gamma$ RRL parameters are given in Table A.2 which lists the source, the transition, the element, the line intensity, the FWHM line width, the LSR velocity, the rms noise in the spectrum, and the total on-source integration time for each position, including the corresponding $1 \sigma$ uncertainties of the Gaussian fits. The averaged $\operatorname{Hn} \beta$ and $\operatorname{Hn} \gamma$ spectra are shown in Figures A.2 and A.3, respectively. 


\subsection{The PDR Boundaries}

Defining the PDR boundaries of the observed H II regions is a crucial step in determining the region properties. Since PAHs are abundant in PDRs where they emit strongly in the $8 \mu \mathrm{m}, 12 \mu \mathrm{m}$, and $24 \mu \mathrm{m}$ bands (e.g., Hollenbach \& Tielens, 1997a), enhancements in the $12 \mu \mathrm{m}$ WISE emission can be used to trace the PDR itself. We define the PDR boundary for all observed regions by following the enhanced $12 \mu \mathrm{m}$ emission surrounding the H II region by hand. We estimate the inner and outer PDR boundary for each region such that the width of the PDR is the FWHM of the enhanced emission. Although this characterization of the PDR structure is by no means unique, results from L16 show that the above method appears to reliably trace the PDR around compact H II regions.

The PDR boundaries are determined using the above method for each of the 8 observed H II regions and the results are shown in Figure 4.1. The term "strong" refers to a PDR boundary for which the enhanced $12 \mu \mathrm{m}$ emission shows the largest contrast to the surrounding medium, whereas a "weak" PDR is barely distinguishable from the $12 \mu \mathrm{m}$ background. Several PDR boundaries are asymmetrical or incomplete in the associated $12 \mu \mathrm{m}$ emission. For example, in the case of G45, the western PDR boundary is much weaker and further away from the region center than the eastern boundary. For M16, the PDR shows discontinuities to the east and west, but is strong toward the north (the sampled directions). Similarly, the PDR boundary of Orion appears to be strongest toward the east and west and less so towards the south where it is at the largest distance from the center.

Since our by-eye definition of the PDR boundaries is subjective and depends on tracing partially weak or incomplete enhancements in $12 \mu \mathrm{m}$ emission, we do not attempt to quantify the uncertainty associated with the PDR location. Tracing the PDR boundary is particularly challenging for G29 due to its very weak $12 \mu \mathrm{m}$ emission, many discontinuities, and proximity to several nearby continuum sources. For M16 and Orion, several PDR components are visible in a given direction. In these cases, we define the strongest PDR boundary as the "main" PDR. For a number of 

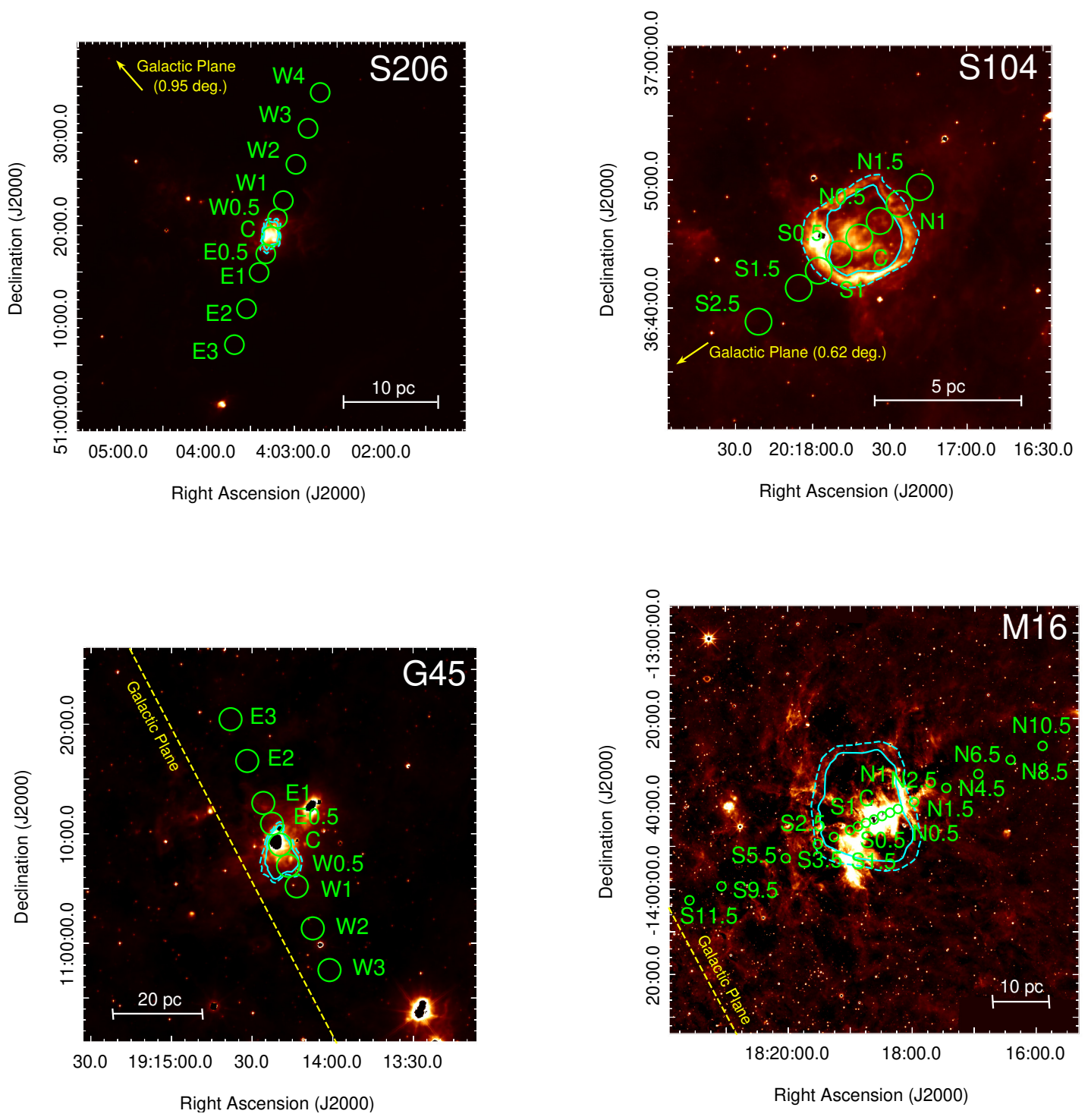

Figure 4.1: The observed H II regions in $12 \mu \mathrm{m}$ WISE emission. This page: S206 (top left), S104 (top right), G45 (bottom left), and M16 (bottom right). The green circles show the positions that we observed with the GBT; the size of the circles corresponds to the average GBT beam width at the observed frequencies. The solid and dashed light blue regions indicate the inner and outer PDR boundaries for each H II region as defined in $\S 4.4$ and the scale bars are derived from the distances given in Table 4.1. The yellow arrow or yellow dashed line indicates the location of the Galactic plane. The angle next to the arrow gives the distance between the central position of the H II region and the Galactic plane. 

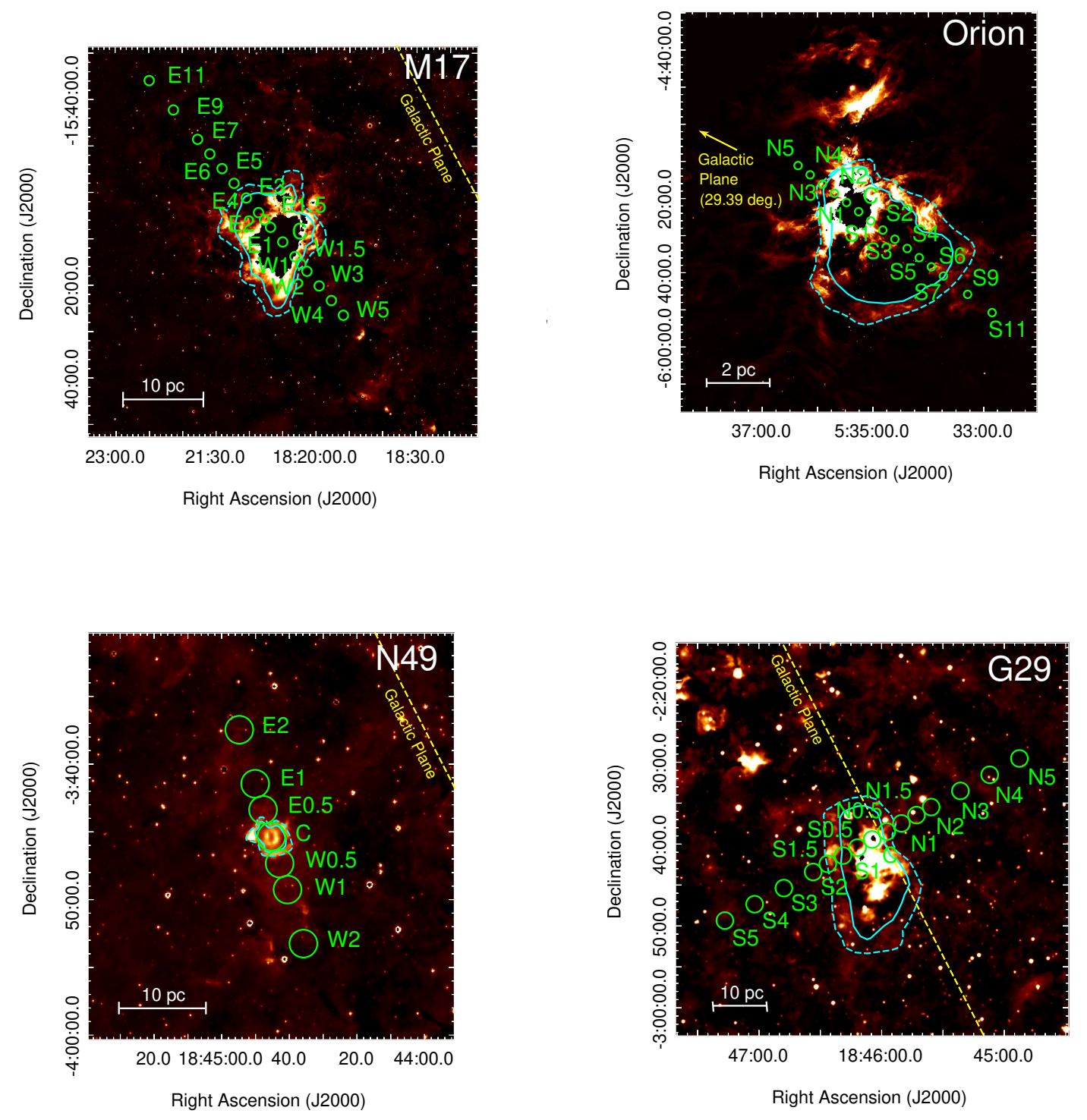

Figure 4.1 (cont.): The observed H II regions in $12 \mu \mathrm{m}$ WISE emission. This page: M17 (top left), Orion (top right), N49 (bottom left), and G29 (bottom right). 
regions, the sharp decrease in $12 \mu \mathrm{m}$ intensity makes it difficult to unambiguously locate the PDR boundary toward some directions.

\subsection{Hydrogen RRL Emission}

Using our GBT data, we test the hypothesis that high luminosity H II regions have a greater effect on maintaining the ionization of the WIM compared to compact H II regions, possibly because they allow a larger fraction of ionizing radiation to escape into the ISM. In Figure 4.2 the hydrogen RRL intensity is shown, averaged over the $\operatorname{Hn} \alpha$ transitions given in $\S 4.3$, and the ionic abundance ratio (see $\S 4.6$ ) as a function of distance from the region center for all observed H II regions. As expected, the RRL intensity decreases with increasing distance for all regions. Regions with more extended PDR boundaries (e.g., M16, M17, and Orion) have more extended emission than the observed compact regions, possibly because they are in a later evolutionary stage. In Figure 4.3 the hydrogen RRL emission is shown for all observed regions with distance, normalized by the radius of the PDR boundary along the given direction. It is striking that all observed H II regions except for Orion and perhaps M17 exhibit roughly the same hydrogen RRL emission gradient.

We also show exponential fits of the form $I=a \times \exp \left(b r_{\mathrm{PDR}}\right)$ (where $I$ is the hydrogen RRL intensity, $r_{\mathrm{PDR}}$ is the normalized distance from the region center, and $a$ and $b$ are the fit parameters) to the data to highlight the similarity between the gradients. We give the derived fit parameters in Table 4.2, which lists the source, $a$, $b$, and the errors in $a$ and $b$, respectively. The decrease of the hydrogen RRL intensity near NGC 7538 (see §2) is similar to that of S104, both in overall intensity and in slope. It is difficult to directly compare the W43 complex studied in $\S 2$ with the eight H II regions in this study. Since the RRL emission near W43 is caused by several ionizing sources, we cannot easily define a single PDR boundary for W43. When

comparing our exponential model to the power-law model used in $\S 2$, there is no significant difference in the fit quality outside the PDR boundaries. The exponential fits, however, are more accurate for positions within the PDR boundaries since the 

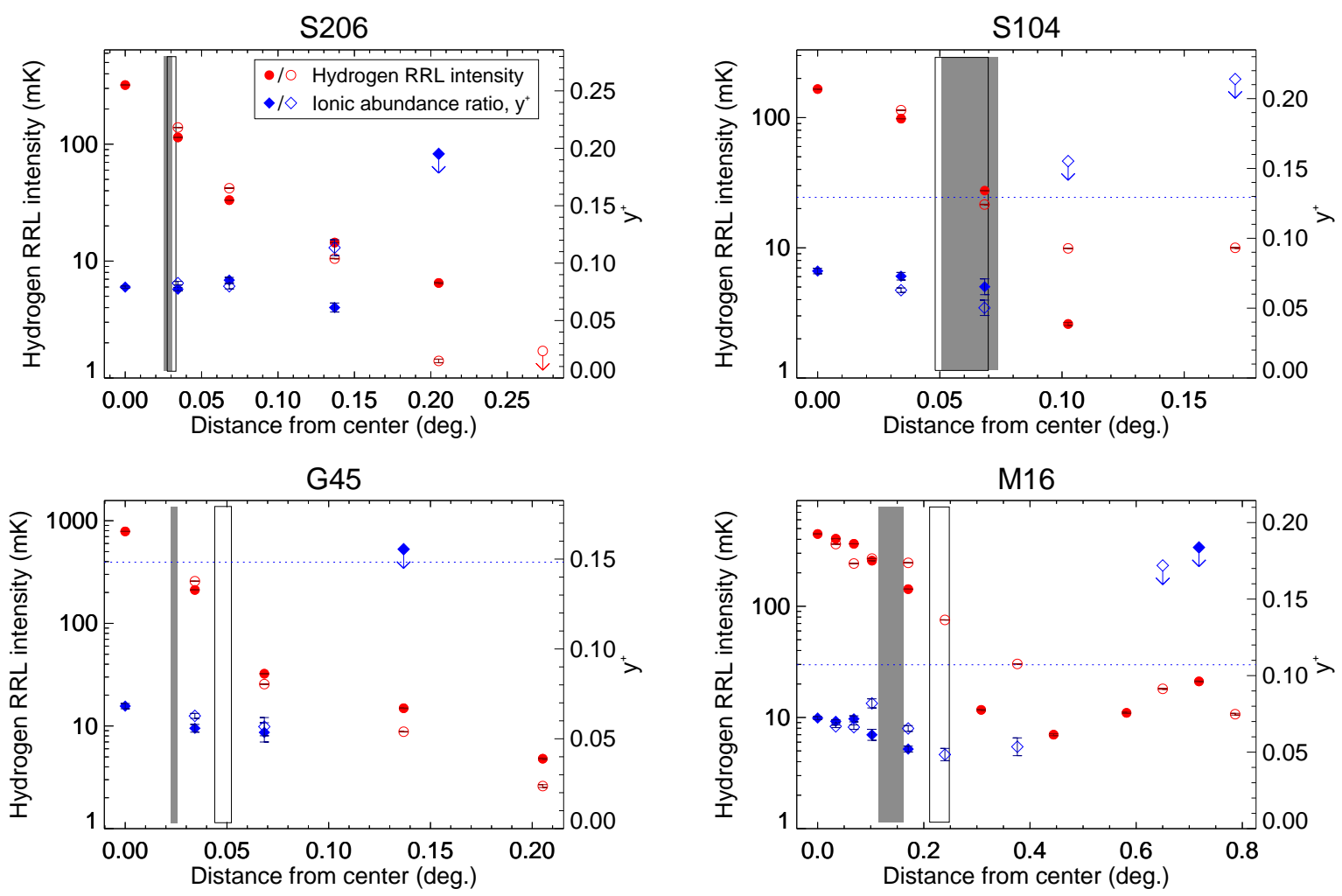

Figure 4.2: The hydrogen RRL intensity (red circles) and ionic abundance ratio $y^{+}$ (blue diamonds) as a function of distance from the region center for all observed H II regions. On this page are shown: S206 (top left), S104 (top right), G45 (bottom left), and M16 (bottom right). The shaded and unshaded symbols indicate pointings towards the two different observed directions from the center (shaded: north/east, unshaded: south/west). Upper limits are denoted as symbols with downward arrows. The large unshaded and shaded regions in the background show the extent of the PDR boundary towards the two directions. The combined upper limit of $y^{+}$for all helium non-detections is marked as the dotted blue horizontal line.

power-law approximation diverges as $r_{\mathrm{PDR}} \rightarrow 0$.

The inverse correlation between H II region size and the slope of the hydrogen RRL intensity may indicate that larger regions allow more ionizing photons to escape through their PDRs which in turn maintain the ionization of the surrounding WIM. Our results suggest that the total amount of escaping ionizing radiation is fundamentally correlated with the radius of the PDR boundary of that region. It is possible that the PDR boundaries surrounding large and luminous H II regions are generally weaker or more inhomogeneous than those surrounding more compact regions. In $\S 4.4$ we show that the PDRs around M16 and Orion are not as well-defined as the PDR 

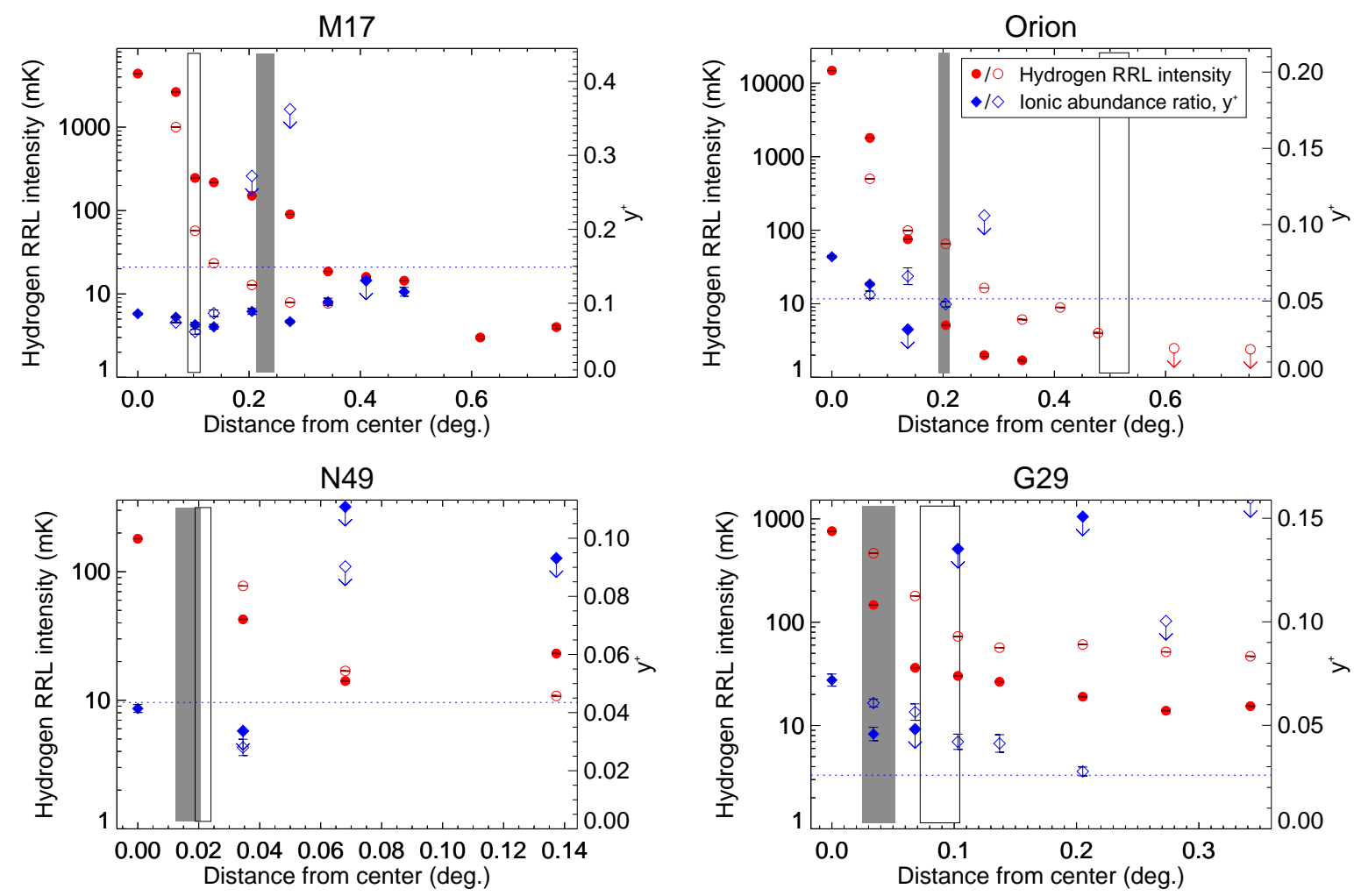

Figure 4.2 (cont.): The hydrogen RRL intensity (red circles) and ionic abundance ratio $y^{+}$(blue diamonds) as a function of distance from the region center for all observed H II regions. On this page are shown: M17 (top left), Orion (top right), N49 (bottom left), and G29 (bottom right). 


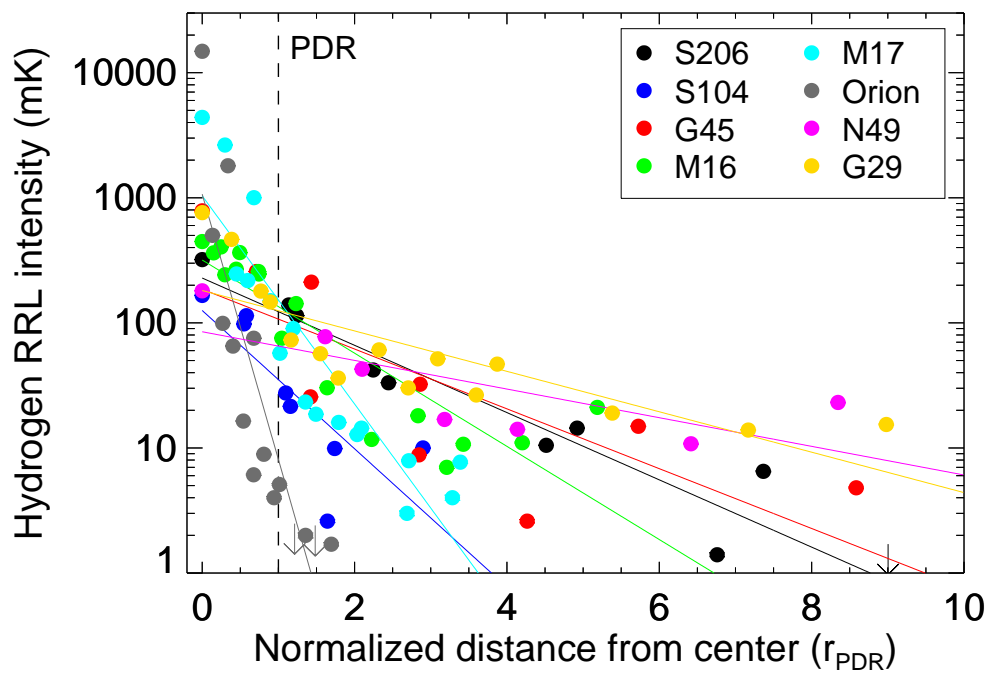

Figure 4.3: The hydrogen RRL intensity for all observed positions as a function of angular offset from the H II regions. The distance is normalized by the radius of the PDR boundary along the observed direction. Downward arrows show upper limits. The solid lines are exponential fits to the data and the vertical dashed line indicates the PDR boundary. This figure shows that, with the exception of Orion and perhaps M17, the hydrogen line intensity decreases with normalized distance at roughly the same rate for all observed $\mathrm{H}$ II regions.

boundaries around the more compact regions in our sample. For M16, this result could be related to the fact that large, high-luminosity H II regions are more likely to be density-bounded rather than radiation-bounded (Beckman et al., 1998), however, the PDR boundaries may be less well-defined for regions with multiple sources of ionization. We note that the observed RRL intensity depends not only on the ionizing flux, but also on the density structure along the line of sight. We make no attempt to separate between the two.

For a number of sources (M16, G29, N49), the intensity does not continue to decrease with distance from the center but rather flattens out beyond a certain radius. We hypothesize that this emission far from the region center is not due to the H II region itself but from the WIM. The observed hydrogen line intensities of $\sim 10-50 \mathrm{mK}$ are not uncommon for WIM emission (see Anderson et al., 2015b; Luisi et al., 2017). It is noteworthy that all these regions are at relatively low Galactic latitudes where presumably emission from the WIM is the strongest (Alves et al., 2012). 
Table 4.2: Fitted Hydrogen RRL Gradients

\begin{tabular}{lrccc}
\hline Source & \multicolumn{1}{c}{$a$} & $\delta a$ & $b$ & $\delta b$ \\
& $(\mathrm{mK})$ & $(\mathrm{mK})$ & & \\
\hline S206 & 228.3 & 23.5 & -0.62 & 0.14 \\
S104 & 125.6 & 16.1 & -1.27 & 0.42 \\
G45 & 185.8 & 18.6 & -0.55 & 0.13 \\
M16 & 318.0 & 19.7 & -0.86 & 0.16 \\
M17 & 1035.6 & 47.3 & -1.92 & 0.24 \\
Orion & 1068.4 & 75.3 & -4.88 & 0.60 \\
N49 & 84.9 & 12.3 & -0.26 & 0.14 \\
G29 & 181.4 & 13.9 & -0.37 & 0.10 \\
\hline
\end{tabular}

Several regions have more than one hydrogen line component. The southern positions of G29 exhibit a second component approximately $-40 \mathrm{~km} \mathrm{~s}^{-1}$ offset from the velocity of the H II region itself. There is also a second hydrogen line visible in N49 and in the southernmost positions of M16. Due to their low RRL intensities and since the existence of an additional $\mathrm{H}$ II region along the line of sight is unlikely, these additional line components are probably due to emission from the WIM (see Anderson et al., 2015b). The second hydrogen component in the central position of M17 is too strong and spatially constrained to be caused by the WIM. It may instead be due to expansion processes within the H II region itself.

\subsection{Ionic Abundance Ratios}

It has been suggested that He-ionizing photons are suppressed as UV photons escape from H II regions (Hoopes \& Walterbos, 2003; Wood \& Mathis, 2004). The complex absorption and re-emission processes in the surrounding gas, however, have never been observed in detail and it is unclear whether this result is applicable to the Galactic H II region population as a whole. The hardness of the interstellar radiation field for our region sample can be constrained by deriving the $y^{+}=N\left({ }^{4} \mathrm{He}^{+}\right) / N\left(\mathrm{H}^{+}\right)$ ionic abundance ratio using our GBT data. Since helium (with an ionization poten- 
tial of $\sim 24.6 \mathrm{eV})$ is ionized by harder radiation compared to hydrogen $(\sim 13.6 \mathrm{eV})$, a larger value of $y^{+}$indicates a more energetic radiation field, assuming that the overall helium-to-hydrogen abundance ratio remains constant.

We calculate $y^{+}$using

$$
y^{+}=\frac{T_{\mathrm{L}}\left({ }^{4} \mathrm{He}^{+}\right) \Delta V\left({ }^{4} \mathrm{He}^{+}\right)}{T_{\mathrm{L}}\left(\mathrm{H}^{+}\right) \Delta V\left(\mathrm{H}^{+}\right)},
$$

where $T_{\mathrm{L}}\left({ }^{4} \mathrm{He}^{+}\right)$and $T_{\mathrm{L}}\left(\mathrm{H}^{+}\right)$are the line temperatures of helium and hydrogen, respectively, and $\Delta V\left({ }^{4} \mathrm{He}^{+}\right)$and $\Delta V\left(\mathrm{H}^{+}\right)$are the corresponding FWHM line widths (Peimbert et al., 1992). For positions with hydrogen but no helium detections, we use upper limits of $T_{\mathrm{L}}\left({ }^{4} \mathrm{He}^{+}\right)=3 \times \mathrm{rms}$ and $\Delta V\left({ }^{4} \mathrm{He}^{+}\right)=\bar{x} \Delta V\left(\mathrm{H}^{+}\right)$, where $\bar{x}$ is the average line width ratio $\Delta V\left({ }^{4} \mathrm{He}^{+}\right) / \Delta V\left(\mathrm{H}^{+}\right)$for the observed region. Our values for $\bar{x}$ range from 0.59 to 0.87 , which is consistent with previous studies by L16 $(\bar{x}=0.84)$ and Wenger et al. $(2013, \bar{x}=0.77 \pm 0.25)$. Since the atomic mass of hydrogen is approximately one-fourth that of helium, $\bar{x}$ should be equal to $\sim 0.5$ in the absence of turbulence. The above values for $\bar{x}$ therefore indicate that turbulence plays a significant role in broadening the observed line widths of our positions (see $\S 4.8$ ).

$y^{+}$is shown for each individual H II region in Figure 4.2 and for all observed regions in Figure 4.4. We observe a decrease in $y^{+}$with distance from the center for most regions. While previous results indicated an approximately constant value of $y^{+}$within the region, and a decrease outside the PDR boundary (L16; see also Balser et al., 2001), our sample shows a relatively steady decrease with angular offset regardless of the sampled location with respect to the PDR. We fit a linear profile, $y^{+}=a+b \times r$ (where $r$ is the distance to the center of the H II region in degrees, and $a$ and $b$ are the fitting parameters), to the ionic abundance ratios of each region, separately for each direction from the region center. We also calculate Spearman's rank correlation coefficient $\rho$, both for each direction separately and for each HII region as a whole. We give the fitting parameters and Spearman's $\rho$ in Table 4.3. 
Table 4.3: Fitted $y^{+}$Gradients and Spearman Coefficients

\begin{tabular}{lccccccc}
\hline Source & Direction & $a$ & $\delta a$ & $\begin{array}{c}b \\
\left(\mathrm{deg}^{-1}\right)\end{array}$ & $\begin{array}{c}\delta b \\
\left(\mathrm{deg}^{-1}\right)\end{array}$ & $\begin{array}{c}\text { Spearman's } \rho \\
\text { (along direction) }\end{array}$ & $\begin{array}{c}\text { Spearman's } \rho \\
\text { (total) }\end{array}$ \\
\hline S206 & East & 0.0798 & 0.0019 & -0.0533 & 0.0238 & -0.400 & +0.202 \\
& West & 0.0788 & 0.0022 & 0.0963 & 0.0288 & +0.800 & \\
S104 & North & 0.0768 & 0.0032 & -0.1408 & 0.0741 & -1.000 & -0.616 \\
& South & 0.0765 & 0.0026 & -0.3947 & 0.0631 & -1.000 & \\
G45 & East & 0.0678 & 0.0027 & -0.2931 & 0.0605 & -1.000 & -0.738 \\
& West & 0.0682 & 0.0022 & -0.1619 & 0.0521 & -1.000 & \\
M16 & North & 0.0735 & 0.0012 & -0.1116 & 0.0120 & -0.900 & -0.743 \\
& South & 0.0705 & 0.0016 & -0.0448 & 0.0088 & -0.750 & \\
M17 & East & 0.0802 & 0.0017 & 0.0087 & 0.0072 & +0.310 & +0.522 \\
& West & 0.0835 & 0.0020 & -0.1330 & 0.0246 & +0.200 & \\
Orion & North & 0.0790 & 0.0009 & -0.2622 & 0.0192 & -1.000 & -0.667 \\
& South & 0.0777 & 0.0013 & -0.1541 & 0.0095 & -0.800 & \\
N49 & East & $\ldots$ & $\ldots$ & $\ldots$ & $\ldots$ & $\ldots$ & -1.000 \\
& West & 0.0414 & 0.0022 & -0.3877 & 0.0920 & -1.000 & \\
G29 & North & 0.0718 & 0.0031 & -0.7658 & 0.1294 & -1.000 & -0.937 \\
& South & 0.0687 & 0.0017 & -0.2042 & 0.0144 & -1.000 & \\
\hline
\end{tabular}

Our results support the hypothesis that a large fraction of He-ionizing photons is being absorbed well within the H II region boundary. We note that the measured $y^{+}$gradient may also partly be due to the geometries of the observed H II regions since we implicitly assume in the derivation of $y^{+}$that both $\mathrm{H}^{+}$and $\mathrm{He}^{+}$fill the beam. We expect to find environments near ionization fronts where hydrogen exists in predominantly ionized form, but where helium remains mostly neutral (see Pankonin et al., 1980). Depending on the geometry of the region, the telescope beam may intersect several ionization fronts, and for these lines of sight the value of $y^{+}$would be lower than expected.

Two regions do not follow the general trend of decreasing $y^{+}$: S206 and M17. S206 shows an increase of $y^{+}$with distance and for M17 the results are inconclusive. While S206 is a relatively compact H II region without much extended emission, M17 is one of the largest and brightest H II regions in the Milky Way. S206 and M17 are the only two known $\mathrm{HII}$ regions with a hard enough radiation field to fully ionize helium within the H II region (see Balser, 2006, and references therein). 

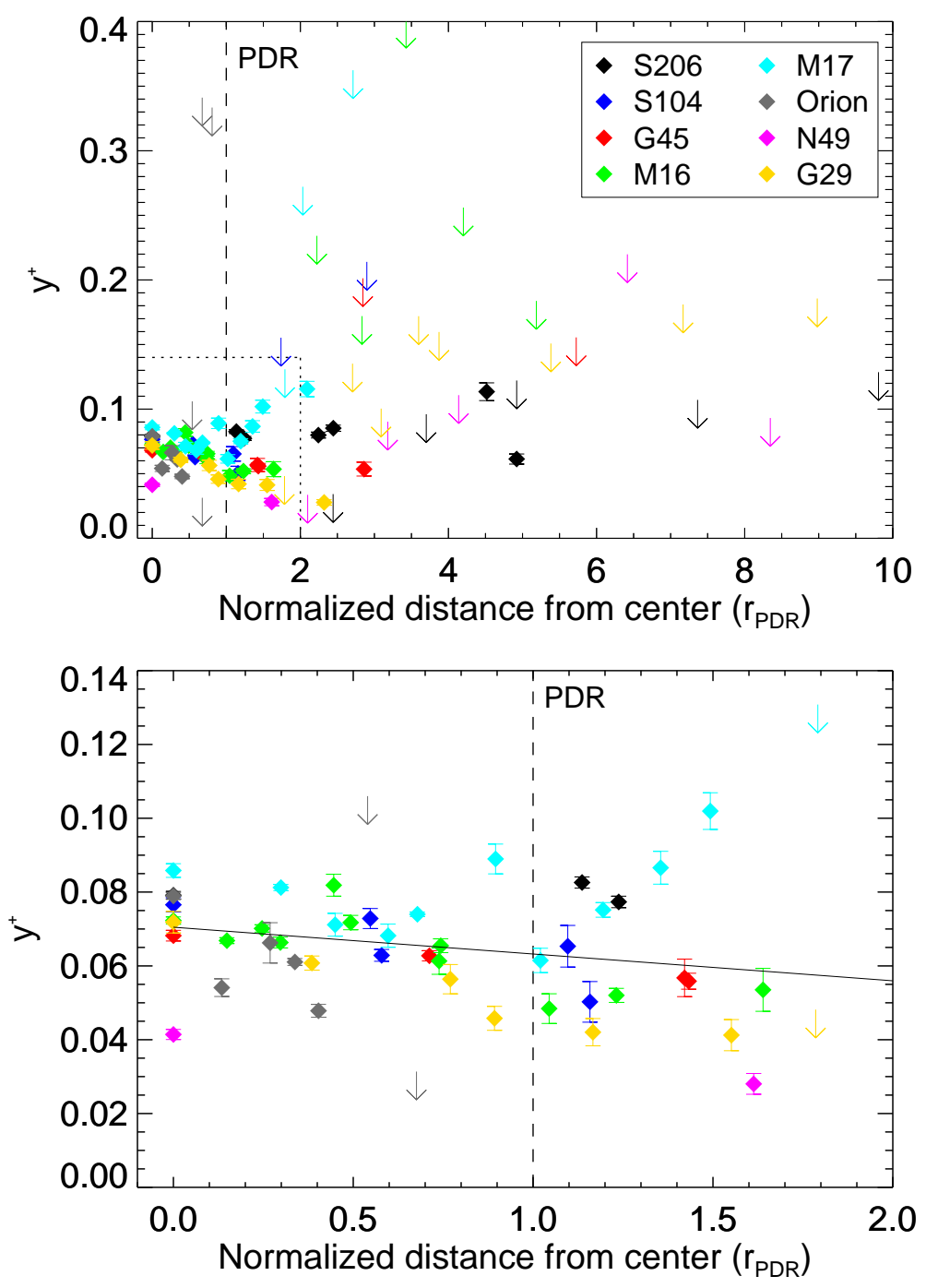

Figure 4.4: Top: The $y^{+}=N\left({ }^{4} \mathrm{He}^{+}\right) / N\left(\mathrm{H}^{+}\right)$ionic abundance ratio as a function of angular offset from the H II region. The distance is normalized by the radius of the PDR boundary along the observed direction. Downward arrows show upper limits and the vertical dashed line indicates the PDR boundary. Except for S206 and M17, $y^{+}$decreases for all regions with distance. Bottom: Same, zoomed into the dotted region shown in the top panel. The solid black line is a linear fit, $y=a+b x$, to the data shown. The fit parameters are $a=0.0705 \pm 0.0036$ and $b=-0.0073 \pm 0.0041$. 


\subsection{Physical Properties of the Ionized Gas}

\subsubsection{LTE Electron Temperatures}

The electron temperature $T_{\mathrm{e}}$ is a proxy for the metallicity of an $\mathrm{H}$ II region (e.g., Rubin, 1985) and can be used to study its intrinsic heating and cooling processes. Previous studies have found conflicting results regarding the relationship between $T_{\mathrm{e}}$ inside and outside the PDR boundaries of HII regions. Most research shows a relatively constant electron temperature distribution within $\mathrm{H}$ II regions (e.g., Roelfsema et al., 1992; Adler et al., 1996; Rubin et al., 2003), but there has been evidence for a decrease of $T_{\mathrm{e}}$ with increasing distance from Orion A (Wilson et al., 2015). Under the assumption of LTE, $T_{\mathrm{e}}$ can be derived by

$$
\left(\frac{T_{\mathrm{e}}^{*}}{\mathrm{~K}}\right)=\left\{7103.3\left(\frac{\nu_{\mathrm{L}}}{\mathrm{GHz}}\right)^{1.1}\left[\frac{T_{\mathrm{C}}}{T_{\mathrm{L}}\left(\mathrm{H}^{+}\right)}\right] \times\left[\frac{\Delta V\left(\mathrm{H}^{+}\right)}{\mathrm{km} \mathrm{s}^{-1}}\right]^{-1} \times\left[1+y^{+}\right]^{-1}\right\}^{0.87},
$$

where $\nu_{\mathrm{L}}=6 \mathrm{GHz}$ is the average frequency of our $\mathrm{Hn} \alpha$ recombination lines, $T_{\mathrm{C}}$ is the continuum antenna temperature, $T_{\mathrm{L}}$ is the $\mathrm{H}$ line antenna temperature, $\Delta V\left(\mathrm{H}^{+}\right)$is the FWHM line width, and $y^{+}$is the ionic abundance ratio from Eq. 4.2 (Mezger \& Ellis, 1968; Quireza et al., 2006).

The derived $T_{\mathrm{e}}^{*}$ are shown in Figure 4.5 for all observed positions where the He line could be detected. While the average electron temperature for each H II region is slightly different, there are no large variations of $T_{\mathrm{e}}^{*}$ with distance for any of the observed regions. For G45, our derived electron temperature of $1240 \pm 1080 \mathrm{~K}$ at a normalized distance of $r_{\mathrm{PDR}}=1.42$ is abnormally low. At this position, our calculated continuum antenna temperature used to derive $T_{\mathrm{e}}^{*}$ is affected by severe baseline instability in our the spectra. We therefore argue that here our value of $T_{\mathrm{e}}^{*}$ does not reflect the actual electron temperature. This assumption is supported by our line profile analysis (§4.8), which yields an estimated electron temperature of $\sim 6000 \mathrm{~K}$ for this direction. We disregard this position for all further analysis. 

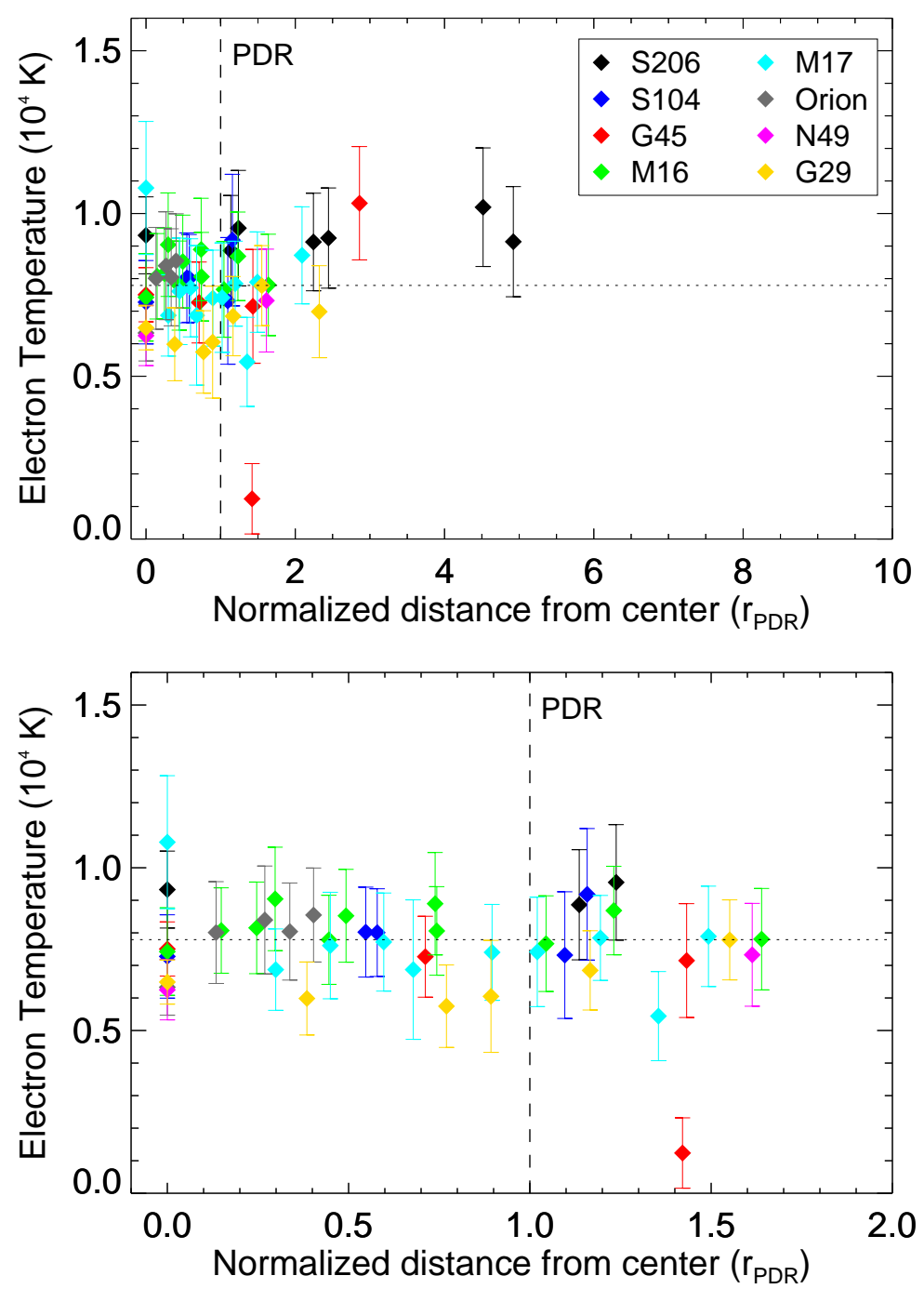

Figure 4.5: Top: The LTE electron temperature $T_{\mathrm{e}}^{*}$ as a function of angular offset from the H II region. The distance is normalized by the radius of the PDR boundary along the observed direction. The vertical dashed line indicates the PDR boundary and the horizontal dotted line shows the average derived electron temperature, $T_{\mathrm{e}}^{*}=$ $7790 \pm 1480$ K. Bottom: Same, zoomed in. 


\subsubsection{Electron Densities}

Assuming that the ionized gas is in LTE, we are able to constrain the emission measure and the root mean square electron number density for each observed direction. The emission measure EM is defined as the integral of the electron density squared $n_{\mathrm{e}}^{2}$ along the line of sight. Because the emission measure is proportional to the optical depth at the line center $\tau_{\mathrm{L}}$, the brightness temperature of a recombination emission line can be estimated by

$$
\frac{T_{\mathrm{b}}}{\mathrm{K}} \approx 1.92 \times 10^{3}\left(\frac{T_{\mathrm{e}}}{\mathrm{K}}\right)^{-3 / 2} \times\left(\frac{\mathrm{EM}}{\mathrm{pc} \mathrm{cm}^{-6}}\right)\left(\frac{\Delta \nu}{\mathrm{kHz}}\right)^{-1}
$$

where $T_{\mathrm{b}}$ is the brightness temperature at the line center and $\Delta \nu$ is the line FWHM (Condon \& Ransom, 2016). Assuming that the RRL emitting region is extended evenly across the GBT beam and using a GBT main beam efficiency of 0.94 at Cband (Maddalena, 2010, 2012), the emission measure can be expressed as

$$
\frac{\mathrm{EM}}{\mathrm{pc} \mathrm{cm}^{-6}} \approx 1.109 \times 10^{-2}\left(\frac{T_{\mathrm{L}}\left(\mathrm{H}^{+}\right)}{\mathrm{K}}\right) \times\left(\frac{\Delta V\left(\mathrm{H}^{+}\right)}{\mathrm{km} \mathrm{s}^{-1}}\right)\left(\frac{T_{\mathrm{e}}}{\mathrm{K}}\right)^{3 / 2} .
$$

We use our LTE electron temperatures $T_{\mathrm{e}}^{*}$ to calculate EM for all positions for which an He line was detected. The resulting EM values range from $270 \mathrm{pc} \mathrm{cm}^{-6}$ to $2.4 \times$ $10^{6} \mathrm{pc} \mathrm{cm}^{-6}$, with the largest values found toward the central directions of Orion and M17 (see Figure 4.6, top panel). All observed H II regions show a decrease in EM with distance from the center, with the exception of M16 whose EM remains roughly constant within the PDR boundaries. The derived EM values are given in Table A.3.

The root mean square electron number density $\bar{n}_{\mathrm{e}}$ can be estimated from the derived emission measures, assuming the H II region geometry is approximated by a slab of constant line-of-sight path length and uniform density. We further assume that the path length for each H II region is twice its radius $R_{\mathrm{PDR}}$ as given in Table 4.1. By definition,

$$
\left(\frac{\mathrm{EM}}{\mathrm{pc} \mathrm{cm}^{-6}}\right)=\int_{\mathrm{los}}\left(\frac{n_{\mathrm{e}}}{\mathrm{cm}^{-3}}\right)^{2} d\left(\frac{s}{\mathrm{pc}}\right)
$$



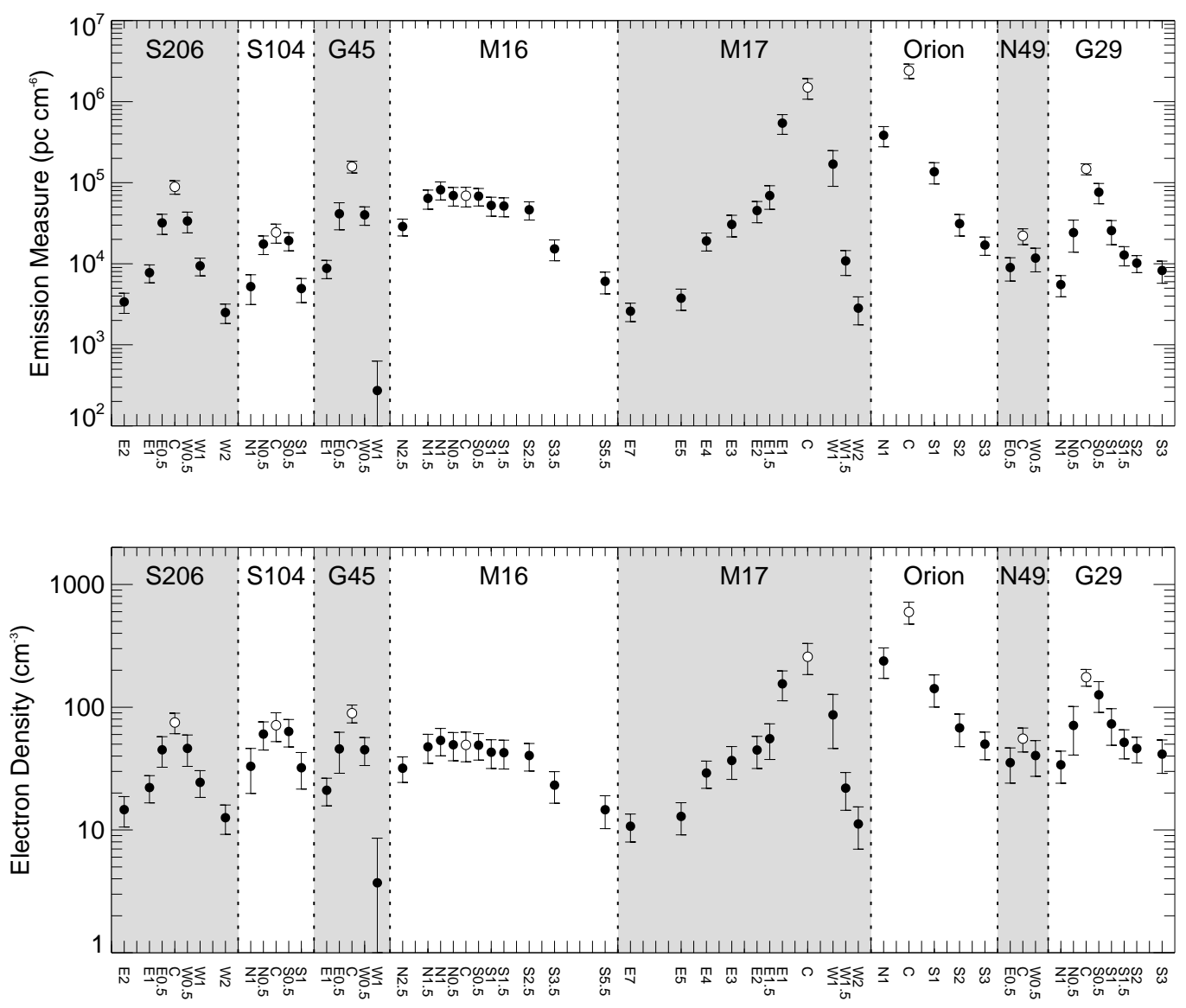

Figure 4.6: Top: The emission measure EM for each observed direction with a $\mathrm{He}$ line detection. The central directions of the $\mathrm{H}$ II regions are indicated by the unfilled circles. The largest EM values are found toward these directions. Bottom: Same, for the root mean square electron density $\bar{n}_{\mathrm{e}}$, assuming a simple slab geometry for each region. 
such that

$$
\frac{\bar{n}_{\mathrm{e}}}{\mathrm{cm}^{-3}} \approx 0.707\left(\frac{\mathrm{EM}}{\mathrm{pc} \mathrm{cm}^{-6}}\right)^{1 / 2}\left(\frac{R_{\mathrm{PDR}}}{\mathrm{pc}}\right)^{-1 / 2} .
$$

We calculate $\bar{n}_{\mathrm{e}}$ for all positions with an He line detection and show the calculated $\bar{n}_{\mathrm{e}}$ values in the bottom panel of Figure 4.6. Due to our assumption of constant path length for each region, $\bar{n}_{\mathrm{e}}$ follows the same trends as EM.

\subsubsection{Non-LTE Analysis}

There is debate in the literature on whether $\mathrm{H}$ II regions and their surroundings are typically close to LTE. In a study of 72 Galactic H II regions, Balser et al. (2011) argue that non-LTE effects, such as stimulated emission should be small within H II regions. Dupree \& Goldberg (1970) show for three Galactic H II regions that the observed intensity ratios of $\mathrm{Hn} \alpha$ and higher-order lines systematically deviate from the theoretical values; in their sample the $\operatorname{Hn} \beta$ to $\operatorname{Hn} \alpha$ line intensity ratio is generally 20$30 \%$ lower than the LTE value. They suggest that this effect is due to departure from LTE and further argue that non-LTE effects are different for each level population (see also Zuckerman et al., 1967). While stimulated emission typically affects $\alpha$-lines more strongly than $\beta$-lines at the same frequency and could account for the lower line ratios, Shaver \& Wilson (1979) suggest that instead the observed line ratios are produced by pressure broadening. They argue that consequently there is no clear evidence of non-LTE effects in single-dish RRL observations of Galactic H II regions (Shaver, 1980).

Unfortunately, our $\beta$ - and $\gamma$-line data cannot be used to test for LTE since we did not detect RRLs in most individual $\operatorname{Hn} \beta$ and $\operatorname{Hn} \gamma$ spectra. While the hydrogen line is detected toward many directions after averaging together the $\beta$ - and $\gamma$-lines, respectively, the averaged spectra are centered at different frequencies than our $\alpha$ lines. Thus, the average beam size varies and a different region of space is sampled for each level population.

The departure from LTE can, however, be directly quantified using our Hn $\alpha$ line data. The following analysis is based on our derived LTE electron temperatures 
(§4.7.1) and root mean square electron densities (§4.7.2). The necessary calculations are from Brocklehurst \& Salem (1977) and Salem \& Brocklehurst (1979), and include the effect of stimulated emission due to an external radiation field, as well as collisional transitions from excited atom-electron collisions. As a first-order approximation, we assume that the conditions toward our observed directions are typical for H II regions but that there is no incident radiation present (see Salem \& Brocklehurst, 1979). Given our average $n=105$ for the observed $\alpha$-lines, we perform a bilinear interpolation between the electron temperature and electron density values from Salem $\&$ Brocklehurst (1979, their Table 1) to find the departure coefficient $b_{\mathrm{n}}$ that matches our values of $T_{\mathrm{e}}^{*}$ and $\bar{n}_{\mathrm{e}} . b_{\mathrm{n}}$ relates the number of atoms in level $n$ to the number that would be there if the system were in thermodynamic equilibrium. Using the same method, we also find the amplification factor $\beta_{\mathrm{n}}$, which describes an enhancement of the stimulated emission due to the overpopulation of level $n$ relative to lower states.

Our values for $b_{\mathrm{n}}$ range from 0.76 to 0.96 and $1-\beta_{\mathrm{n}}=k T_{\mathrm{e}} d\left(\ln b_{\mathrm{n}}\right) / d E_{\mathrm{n}}$ ranges from 46 to 136 . With average $b_{\mathrm{n}}=0.86 \pm 0.04$, there is only a small deviation from LTE for all observed directions. We notice a trend of decreasing $b_{\mathrm{n}}$ with increasing distance from the all H II regions in our sample. This effect is particularly strong for the more luminous H II regions (e.g., M17) and is likely due to the steep decrease in electron density while $T_{e}^{*}$ remains roughly constant. The largest $b_{\mathrm{n}}$ values are found toward the central positions of M17, Orion, and G29, suggesting that due to the large electron densities the collision rates dominate the level populations at these locations.

We note that our non-LTE analysis of the observed positions is only a rough approximation due to the following two reasons. First, $T_{\mathrm{e}}^{*}$ and $\bar{n}_{\mathrm{e}}$ are mean values averaged along the line of sight and over the beam. Given our large average beam $\left(\mathrm{HPBW} \approx 123^{\prime \prime}\right)$ and the H II region geometries, many different physical environments must contribute to these parameters for each observed position. This becomes especially important near the central locations and the PDRs of each H II region where the spatial gradients of electron temperature and density are presumably the steepest. Second, we use $T_{\mathrm{e}}^{*}$ and $\bar{n}_{\mathrm{e}}$ to estimate $b_{\mathrm{n}}$ and $\beta_{\mathrm{n}}$. Since $T_{\mathrm{e}}^{*}$ and $\bar{n}_{\mathrm{e}}$ are based on the assumption of LTE, this approach is only valid for positions that are near LTE. 
While our derived $b_{\mathrm{n}}$ and $\beta_{\mathrm{n}}$ values assume constant density and temperature, and are therefore not truly representative of the values in real H II regions, we argue that $T_{\mathrm{e}}^{*}$ is a reasonable approximation of the true electron temperature averaged along the line of sight and over the HPBW. The line opacities are small for these sources at the observed frequencies, which decreases the impact of stimulated emission. Therefore, $b_{\mathrm{n}}$ provides a good estimate of the deviation from LTE despite typical values of $\geq 100$ for $\beta_{\mathrm{n}}$ (see, e.g., Salem \& Brocklehurst, 1979). With $b_{\mathrm{n}}$ close to unity for all observed positions, it is unlikely that the true average electron temperatures deviate significantly from $T_{\mathrm{e}}^{*}$.

\subsection{Line Profile Analysis}

The observed line widths of RRLs within and near H II regions predominantly depend on two variables: the temperature of the plasma (thermal line broadening) and the amount of turbulence in the local ISM (turbulent line broadening). Other mechanisms that affect the observed line widths, such as natural broadening or pressure broadening, are thought to be negligible given our observed frequencies and electron densities (Hoang-Binh, 1972).

Here we assume that the RRL widths are only affected by thermal broadening and turbulent broadening. Therefore,

$$
\Delta V_{\mathrm{H}}=2.355\left(\frac{k T_{\mathrm{e}}}{m_{\mathrm{H}}}+V_{\text {turb }}^{2}\right)^{1 / 2}
$$

where $\Delta V_{\mathrm{H}}$ is the observed FWHM of the hydrogen RRL, $T_{\mathrm{e}}$ is the electron temperature, $m_{\mathrm{H}}$ is the mass of the hydrogen atom, and $V_{\text {turb }}$ is the velocity contribution due to turbulence. The first term in the parentheses thus corresponds to the thermal contribution, while the constant of 2.355 accounts for the conversion from the onedimensional velocity dispersion to FWHM. Since we can measure $\Delta V_{\mathrm{H}}$ directly, only two unknowns remain, $T_{\mathrm{e}}$ and $V_{\text {turb }}$. These, however, can be expressed in terms of each other by observing the helium RRL toward the same direction, as long as $T_{\mathrm{e}}$ and $V_{\text {turb }}$ are unchanged between the two species. After accounting for natural constants 
and atomic masses,

$$
\left(\frac{T_{\mathrm{e}}}{\mathrm{K}}\right)=29.22\left[\left(\frac{\Delta V_{\mathrm{H}}}{\mathrm{km} \mathrm{s}^{-1}}\right)^{2}-\left(\frac{\Delta V_{\mathrm{He}}}{\mathrm{km} \mathrm{s}^{-1}}\right)^{2}\right] \text {, }
$$

where $\Delta V_{\mathrm{He}}$ is the observed FWHM of the helium RRL.

Our results for $T_{\mathrm{e}}$ are shown in Figure 4.7. As for $T_{\mathrm{e}}^{*}$, only directions for which the helium line was detected are included. The average electron temperature is $T_{\mathrm{e}}=7940 \pm 4720 \mathrm{~K}$ outside the H II region PDRs and $T_{\mathrm{e}}=9180 \pm 3950 \mathrm{~K}$ within. As for $T_{\mathrm{e}}^{*}$, this difference is not statistically significant. The larger deviation between individual values of $T_{\mathrm{e}}$ suggests that this method of calculation is less robust than that described in $\S 4.7 .1$. This may be due to the strong dependence of $T_{\mathrm{e}}$ on the width of the observed hydrogen and helium RRLs, and the fact that $\Delta V_{\mathrm{He}}$ may have larger uncertainties than those given in Table A.2 because of its low intensity and sensitivity to baseline variations. Due to the large deviation between individual values of $T_{\mathrm{e}}$, we use $T_{\mathrm{e}}$ only as a consistency check in this work.

\subsection{Carbon RRLs}

Previous results suggest that carbon RRL emission is often observed from H II region PDRs (e.g., Hollenbach \& Tielens, 1999, L16). This is possibly due to its first ionization potential of only $\sim 11.3 \mathrm{eV}$, which is lower than that of hydrogen or helium. The carbon in H II region PDRs may therefore be ionized by soft-UV photons $(E<13.6 \mathrm{eV})$ that pass through the H II region essentially undisturbed, aside from being attenuated by dust.

We do not observe enhanced carbon RRL emission near the PDR boundaries for most H II regions in our sample (see top panel of Figure 4.8). Instead, the emission is strongest within the HII regions and decreases steadily with distance. While it is likely that a fraction of the PDR is contained within the telescope beam along the line of sight, this may also suggest that a large number of soft-UV photons are attenuated by dust present within the H II regions. S104 is the only observed source for which the 

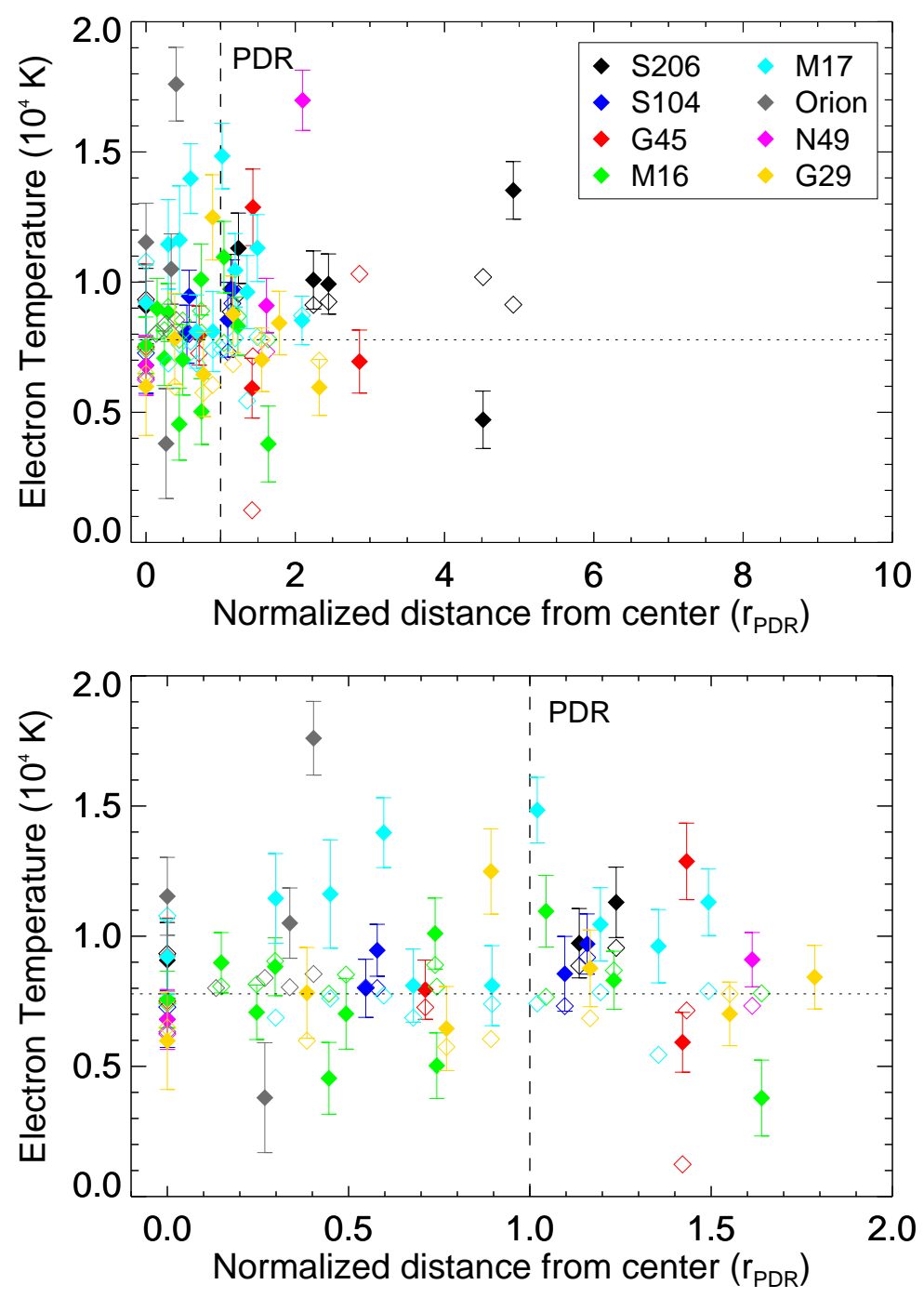

Figure 4.7: Top: The electron temperature derived from our line profile analysis $T_{\mathrm{e}}$ (filled symbols) as a function of angular offset from the H II region. Shown with the unfilled symbols is the LTE electron temperature derived in $\S 4.7 .1$. The distance is normalized by the radius of the PDR boundary along the observed direction. The vertical dashed line indicates the PDR boundary and the horizontal dotted line shows the average derived LTE electron temperature from Figure 4.5. Bottom: Same, zoomed in. 
line emission may be increased near the PDR. The large number of non-detections for S104, however, casts doubt on the statistical significance of this interpretation.

At low frequencies, carbon can be observed in either absorption lines or amplified emission lines due to stimulated emission from inverted populations. Observations of Cas A at $26 \mathrm{MHz}$ show spectral features consistent with the detection of a carbon- $\alpha$ absorption line at $n \sim 630$ (Konovalenko \& Sodin, 1981; Walmsley \& Watson, 1982). In an Ooty Radio Telescope study of RRLs near $327 \mathrm{MHz}$, Roshi et al. (2002) found evidence of stimulated emission, resulting a strong correlation between carbon RRL intensity and continuum emission.

To test whether carbon RRL emission is amplified by stimulated emission at our higher average observing frequency of $\sim 6 \mathrm{GHz}$, we show the carbon RRL intensity as a function of continuum intensity in the bottom panel of Figure 4.8. While there is a correlation between the carbon emission and the continuum intensity, the spread in our data is quite large. Most of the spread, however, is caused by only two sources, Orion and M17, which show stronger carbon RRL emission than expected given their background continuum intensity. These two sources are among the largest and most luminous H II regions in our sample. This suggests that much of the carbon emission may be amplified by stimulated emission. At large distance offsets, a possible lack of amplification of the carbon lines by stimulated emission may explain our carbon non-detections where the continuum emission is weak. It is also possible that a low carbon abundance in the diffuse medium at these positions is responsible for the non-detections.

\section{$4.10 \mathrm{He}^{++}$Emission}

The second ionization potential of helium at $\sim 54.4 \mathrm{eV}$ exceeds helium's first ionization potential by over a factor of two and thus $\mathrm{He}^{+}$is ionized by only the most energetic radiation fields within H II regions. Previous GBT observations by Roshi et al. (2017) failed to detect emission from $\mathrm{He}^{++}$in the diffuse gas surrounding ultracompact H II (UCH II) regions. Their combined $1 \sigma$ upper limit for their setup is 

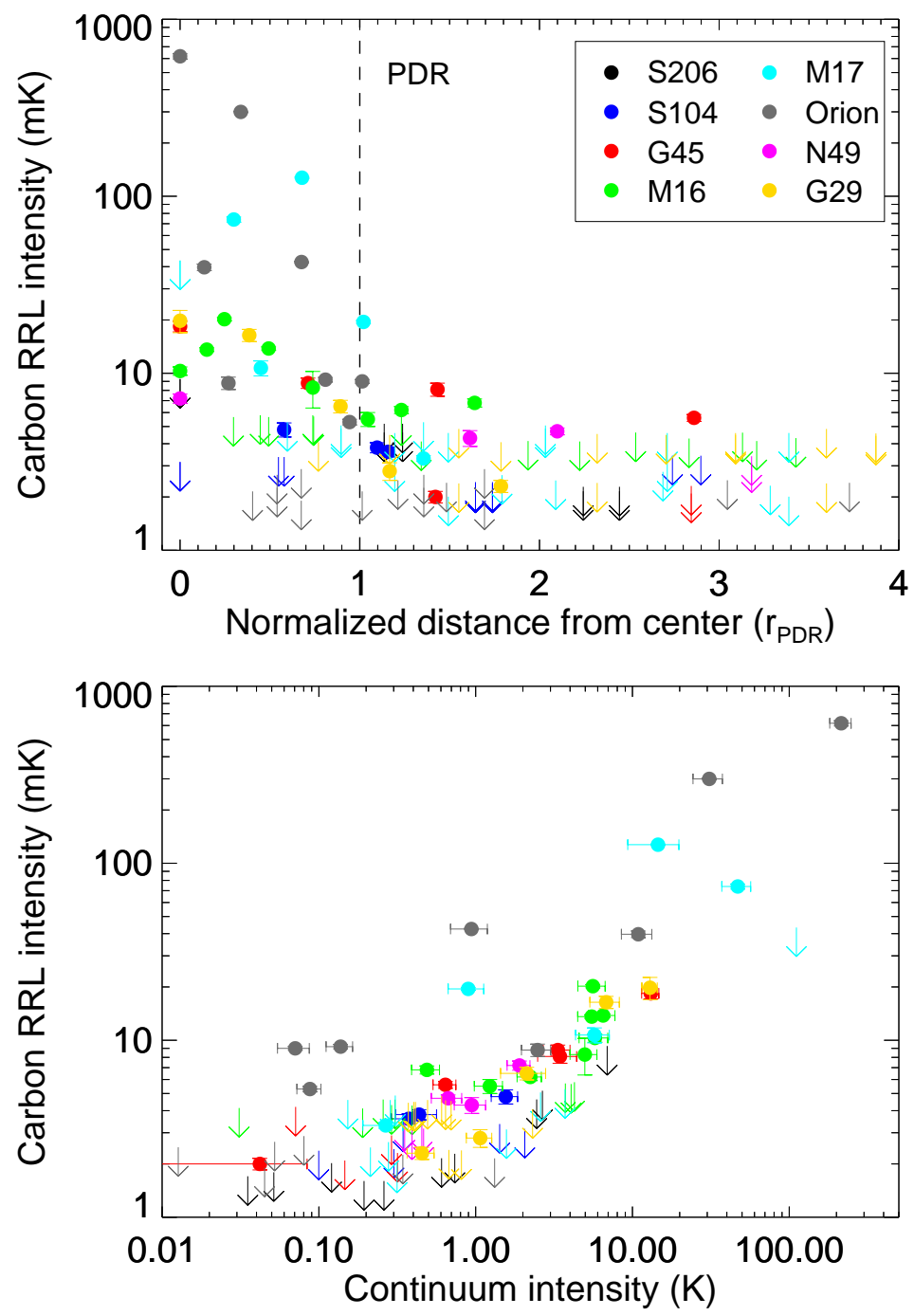

Figure 4.8: Top: The carbon line intensity as a function of angular offset from the H II region. The distance is normalized by the radius of the PDR boundary along the observed direction. Downward arrows show upper limits and the vertical dashed line indicates the PDR boundary. There is no increase in the carbon intensity near the PDR boundaries. Bottom: Correlation between the carbon line intensity and the continuum intensity. The correlation may indicate that our carbon non-detections at large distance offsets are due to a lack of amplification by stimulated emission or that the carbon abundance in the diffuse medium is low. 
$4 \mathrm{mK}$, however, they only observed $3 \mathrm{UCH}$ II regions with a total of 18 independent pointings.

Although many of our sampled positions are cospatial with luminous H II regions, we did not detect the $\mathrm{He}^{++}$line. The rms values for our individual positions range from 3 to $20 \mathrm{mK}$, corresponding to upper limits for the $\mathrm{He}^{++}$line of 9 to $60 \mathrm{mK}$. Our best upper limit for the $\mathrm{He}^{++} / \mathrm{He}^{+}$line ratio is 0.064 , sampled at the central position of Orion. We average together all positions for which the line intensity of singly-ionized $\mathrm{He}$ is at least $30 \mathrm{mK}$ after shifting them in velocity, and again fail to detect the $\mathrm{He}^{++}$line. The rms of our combined, averaged spectrum is $0.6 \mathrm{mK}$, corresponding to a $3 \sigma$ upper limit for the $\mathrm{He}^{++}$line of $1.8 \mathrm{mK}$.

\subsection{Conclusions}

Despite our recent studies (Anderson et al., 2015, L16), radiation leaking from Galactic H II regions is still not well-understood. Models and observations of external galaxies suggest that 30-70\% of the emitted ionizing radiation escapes from H II regions into the ISM (Oey \& Kennicutt, 1997; Zurita et al., 2002; Giammanco et al., 2005; Pellegrini et al., 2012). Due to observational constraints, however, these studies tend to be biased towards the largest and most luminous H II regions which only make up a small fraction of the total number of $\mathrm{H}$ II regions in a given galaxy. Here we observe a sample of Galactic H II regions of various sizes, geometries, and luminosities, including several compact (radius $<5 \mathrm{pc}$ ) H II regions whose extragalactic counterparts may have been missed in previous studies.

If ionizing radiation leaking from an individual $\mathrm{H}$ II region is responsible for maintaining the ionization of the WIM around that region, we expect a decrease in RRL intensity with distance from the region, for which the slope of the decrease is believed to roughly constrain the amount of leaking photons (see L16). We observe a power-law decrease in hydrogen RRL emission intensity outside the PDR of all observed H II regions (see Figure 3 ). We showed in $\S 4.5$ that the hydrogen line intensity decreases with roughly the same slope for all our targets except Orion when 
normalizing the angular offset from the region center by the radius of the PDR. This suggests that the slope in the decrease of hydrogen RRL intensity with distance is directly related to the size of the region. The case of Orion is further complicated by its highly asymmetrical geometry and the presence of several PDR boundaries toward the observed directions. In fact, when defining the innermost visible enhancement in $12 \mu \mathrm{m}$ emission as the main PDR boundary, its slope is similar to that of the other sources, suggesting that the power-law decrease of hydrogen RRL intensity with distance and region size may fundamentally be the same for all Galactic H II regions. The physical implications of this result are unclear, although it may be related to a transition from ionization-bounded to density-bounded behavior (e.g., Rozas et al., 1998; Zurita et al., 2002).

Using the ionic abundance ratio $y^{+}$, the hardness of the interstellar radiation field can be constrained for our region sample. We observe a general trend of decreasing $y^{+}$with distance from the H II regions. This is indicative of absorption of He-ionizing photons within the ionization front of the H II region. It is unclear why two of the observed regions, S206 and M17, do not follow this trend. The difference in their physical properties, such as M17 being ionized by more than one O star, makes it unlikely that this is due to the region's size, luminosity, or morphology.

The electron temperature $T_{\mathrm{e}}$ is believed to remain relatively constant within H II regions (Roelfsema et al., 1992; Adler et al., 1996; Rubin et al., 2003), although recently Wilson et al. (2015) reported on a decrease in $T_{\mathrm{e}}$ with increasing distance from Orion A. We calculate the electron temperature for our positions with He detections using two independent methods. The first method assumes that LTE is satisfied for all observed directions (see also Mezger \& Ellis, 1968; Quireza et al., 2006). We find no general trends in the derived electron temperatures with distance from the $\mathrm{H}$ II regions and no statistically significant difference between $T_{\mathrm{e}}$ within and outside the $\mathrm{H}$ II region PDRs. The second method is based on a line profile analysis and assumes that $T_{\mathrm{e}}$ and the amount of turbulent line broadening remain the same between hydrogen and helium. Although LTE is not required, this method appears to be less robust than the first, possibly due to the larger contribution of line width uncertainties on the 
overall uncertainty in $T_{\mathrm{e}}$. We, again, do not detect a significant difference between electron temperatures within and outside the H II region PDRs.

Our H II region sample spans a wide range of emission measures and root mean square electron densities $\bar{n}_{\mathrm{e}}$. While most observed directions have $10^{2} \lesssim \bar{n}_{\mathrm{e}} \lesssim$ $10^{3} \mathrm{~cm}^{-3}$, the electron densities at the central locations of M17 and Orion are as large as $258 \mathrm{~cm}^{-3}$ and $596 \mathrm{~cm}^{-3}$, respectively. These values are significantly lower than those found by optical line tracers (e.g., Danks, 1970; Kitchin, 1987), possibly because they are based on $T_{\mathrm{e}}^{*}$ averaged along the line of sight and over the HPBW. With an average departure coefficient of $b_{\mathrm{n}}=0.86 \pm 0.04$, we do not find strong departure from LTE in our sample. We note that $b_{\mathrm{n}}$ is largest toward the high- $\bar{n}_{\mathrm{e}}$ regions at the central locations of our H II regions sample where the level populations are dominated by collisions. As expected, $b_{\mathrm{n}}$ decreases with increasing distance from all observed H II regions, suggesting that non-LTE effects become more significant in the lower-density envelopes surrounding Galactic H II regions.

Finally, we do not find enhanced carbon RRL emission near the PDR boundaries, as has been observed previously (Hollenbach \& Tielens, 1999, L16). This may indicate selective attenuation of soft-UV photons by dust within the $\mathrm{HI}$ regions. However, there exists a correlation between carbon RRL intensity and continuum intensity. This suggests that the carbon emission is amplified by stimulated emission. 


\section{Chapter 5}

\section{Current and Future Plans}

Our current work focuses on constraining the large-scale distribution of the WIM, analyzing its physical properties, and determining how leaking photons from H II regions affect the WIM across all spatial scales. The GBT Diffuse Ionized Gas Survey (GDIGS) is a large, ongoing GBT project to map the WIM toward the first Galactic quadrant in RRL emission. These observations, in combination with complementary data (e.g., dust temperature maps from the Herschel Space Observatory), will allow us for the first time to provide a census of the inner-Galaxy WIM unaffected by emission from discrete H II regions and further study the connection between H II regions and the WIM.

\subsection{The GBT Diffuse Ionized Gas Survey}

GDIGS is an RRL and radio continuum survey at C-band (4-8 GHz) using the GBT. Its advantage compared to previous RRL surveys is that we can tune to 64 spectral windows simultaneously, including $22 \mathrm{Hn} \alpha$ lines, $25 \mathrm{Hn} \beta$ transitions, and eight $\mathrm{Hn} \gamma$ transitions. We also tune to eight molecular lines, including $\mathrm{CH}_{3} \mathrm{OH}$ and $\mathrm{H}_{2} \mathrm{CO}$. We then average together all $\mathrm{Hn} \alpha, \mathrm{Hn} \beta$, and $\mathrm{Hn} \gamma$ lines, respectively, making our data the most sensitive large-scale RRL maps extant. The sensitivity is sufficient to detect $\sim 0.1 \mathrm{~cm}^{3}, \sim 10^{4} \mathrm{~K}$ plasma, so long as its path length is $\gtrsim 1 \mathrm{kpc}$ and its emission fills the telescope beam.

The full survey range of GDIGS includes the inner-Galaxy midplane from $32.3^{\circ}>$ $\ell>-5^{\circ}$ and $|b|<0.5^{\circ}$, as well as some explorations above and below the Galactic plane around active star formation regions (see Figure 5.1). At the time of writing (April 2019), GDIGS observations are 85\% complete and we have finalized the data reduction pipeline. 


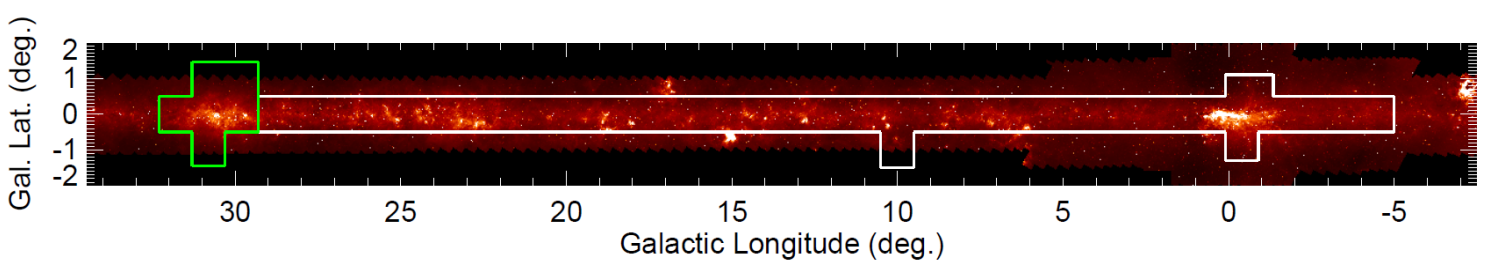

Figure 5.1: Full survey range of GDIGS (shown in white). The background image is of Planck $100 \mathrm{GHz}$ data of the Galactic plane from $34^{\circ}>\ell>-7^{\circ}$. The W43 complex is shown in green.

\subsubsection{Science Goals of GDIGS}

A major science goal of GDIGS is to determine the distribution and dynamical state of the inner-Galaxy WIM. We have performed a similar analysis in $\S 3$, however, our maps in $\S 3$ are heavily interpolated between relatively few discrete telescope pointings. The GDIGS maps are Nyquist-sampled and therefore provide an accurate census of the WIM along all lines of sight. By associating WIM components with spiral arm features in velocity space, we can potentially resolve the KDA and estimate the 3-dimensional distribution of the WIM. We will also create "WIM-only" datacubes that are devoid of emission from discrete HII regions based on the locations and angular sizes of H II regions in the WISE Catalog of Galactic H II Regions.

GDIGS will also allow us to better constrain changes in the radiation field within the diffuse ISM. We know that He-ionizing photons are suppressed as the radiation from individual OB stars escapes through their surrounding PDRs (see $\S 4$ ), but from our data it is not fully clear where this transition takes place and how it is related to the size, age, and morphology of the H II region. With the GDIGS data, we can derive the $N\left({ }^{4} \mathrm{He}^{+}\right) / N\left(\mathrm{H}^{+}\right)$ionic abundance ratio for a large variety of environments to understand what mechanisms may be responsible for the radiation softening.

Using the "WIM-only" datacube, we can investigate the fraction of ionized radiation leaking from H II regions. Within the GDIGS survey range are $\sim 2500 \mathrm{HII}$ regions and $\mathrm{H}$ II region candidates. Compared to $\S 2$ and $\S 4$, the increased sample size will allow us to study regions of all sizes, ages, and luminosities. We can then use these data to estimate the global leaking fraction of the Milky Way. As part of this 
analysis, we will create a model to determine the parameters of H II region photon escape that best match the observed WIM distribution.

Finally, we will study the impact that leaking radiation has on dust emission. In $\S 2$ and $\S 3$ we show that low energy photons must also be escaping from H II regions, which can heat the surrounding dust without destroying it. We can compute dust temperature maps from public Herschel data. Similar to the H II region escape fraction model, we can create a model for the Galaxy's dust temperature distribution based on the WISE catalog. By comparing the model with the observed dust temperatures, we can estimate the global effect of local heating by H II regions.

\subsubsection{The HiI Region Complex W43}

We have fully reduced and have begun to analyze our GDIGS observations of the H II region complex W43 at $\ell \sim 31^{\circ}$ (the region marked in green in Figure 5.1). Our maps encompass six square degrees around W43. These data are ideal for testing the methods described in $§ 5.1 .1$ on a smaller scale.

We show our RRL moment 0 data of W43 in Figure 5.2. The strongest RRL emission comes from the large star-forming clusters W43 (W43-Main) at $\ell \sim 30.8^{\circ}$ and G29 (W43-South) at $\ell \sim 29.9^{\circ}$. There is significant emission from the diffuse gas not associated with any discrete $\mathrm{H}$ II region out to $|b| \sim 1^{\circ}$. In particular, we detect an angularly large, low-intensity structure below the Galactic plane that extends downward from W43 to $b \sim-1^{\circ}$. The structure may be associated with the "wormionized medium" (e.g., Heiles et al., 1996), low-density ionized gas within filamentary structures extending outwards from the Galactic plane. Such worms may act as pathways for Lyman continuum photons to escape from the mid-plane and reach the observed WIM scale height of $\sim 1 \mathrm{kpc}$.

In order to study the WIM emission unaffected by emission from discrete H II regions, we must disentangle the RRL emission based on its origin. We blank emission from within known HII regions and H II region candidates based on their locations, angular sizes, and velocities from the WISE Catalog of Galactic H II Regions. For confirmed H II regions, we only exclude data near the velocity of the region, while for 

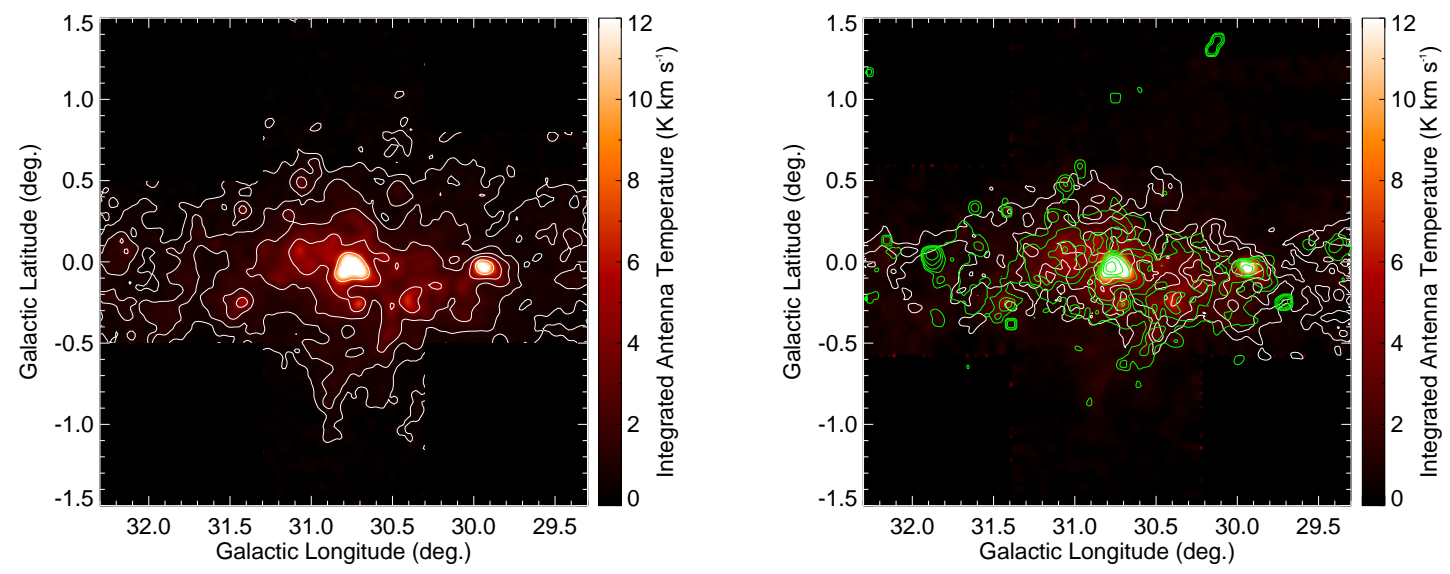

Figure 5.2: Left: moment 0 RRL map of the W43 complex, integrated from 10$130 \mathrm{~km} \mathrm{~s}^{-1}$. Contours are at $0.5,1,2,4$, and $8 \mathrm{~K} \mathrm{~km} \mathrm{~s}^{-1}$. The emission at $\ell \sim 30.8^{\circ}$ is due to W43 itself (W43-Main), while G29 (W43-South) is at $\ell \sim 29.9^{\circ}$. Data at $\ell<$ $30.3^{\circ}$ and $b>0.8^{\circ}$ were blanked due to a bad baseline. Essentially all visible emission is due to hydrogen. Right: same, but the white contours are of integrated ${ }^{13} \mathrm{CO}$ main beam brightness temperature, logarithmically spaced from 7.5 to $120 \mathrm{~K} \mathrm{~km} \mathrm{~s}^{-1}$. The green contours are of VGPS $1.4 \mathrm{GHz}$ continuum emission and are logarithmically spaced between 5 and $320 \mathrm{~K}$. The bright VGPS regions near $(\ell, b)=\left(29.7^{\circ},-0.3^{\circ}\right)$ and $\left(31.7^{\circ},+0.0^{\circ}\right)$ not associated with strong RRL emission are due to supernova remnants (e.g., Green, 2014). 


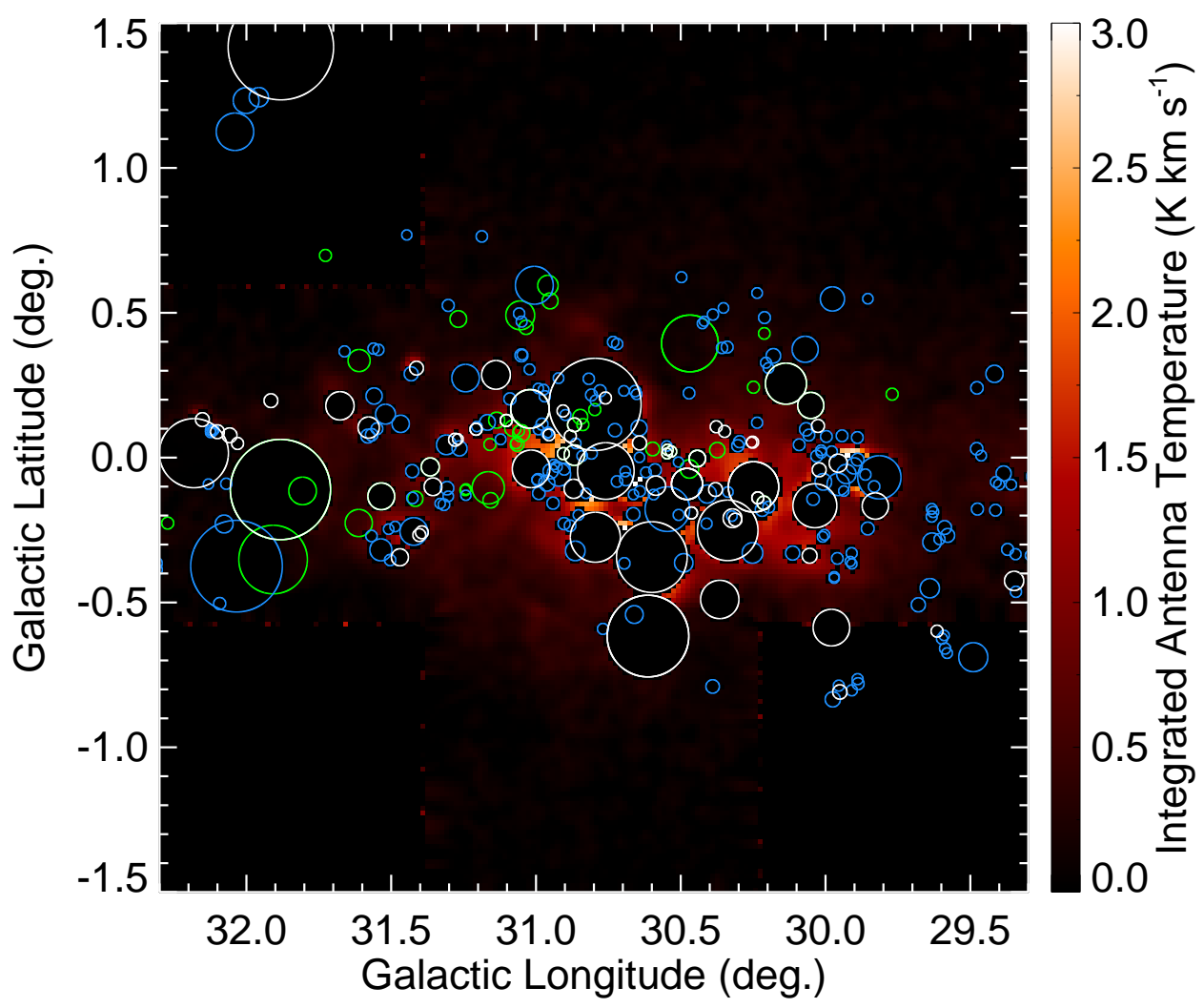

Figure 5.3: Diffuse RRL emission, integrated from $90 \mathrm{~km} \mathrm{~s}^{-1}$ to $110 \mathrm{~km} \mathrm{~s}^{-1}$. Emission from known H II regions within these velocities (white circles) was cut from the map. We also removed emission from H II region candidates (blue circles). The green circles indicate H II regions outside the $90 \mathrm{~km} \mathrm{~s}^{-1}$ to $110 \mathrm{~km} \mathrm{~s}^{-1}$ velocity range.

H II region candidates we blank all velocity channels. We show an example WIM-only map in Figure 5.3.

Based on the WIM-only moment 0 map of W43, we create an empirical model of leaking emission from H II regions. Model inputs are the location and size of each H II region, and its estimated RRL flux density. We assume that the slope of the hydrogen emission with distance from each H II region is roughly inversely proportional to the H II region size traced by its PDR (see $\S 4$ ). We leave as free parameters the average slope of the hydrogen emission with distance from each region, and the fraction of ionizing radiation that escapes from the regions. We assume that the escape fraction is related to the RRL intensity of each H II region, such that $f_{\text {esc }} \propto T_{\mathrm{A}}^{c}$, where $T_{\mathrm{A}}$ is 
the average antenna temperature of the region and $c$ is left as a free parameter. Thus,

$$
T_{\mathrm{A}, \text { model }}=a \sum_{i=1}^{N}\left(T_{\mathrm{A}, i}\right)^{c+1}\left(\frac{r_{i}}{r_{\mathrm{PDR}, i}}\right)^{-b}
$$

where $T_{\mathrm{A} \text {, model }}$ is the antenna temperature of the model, $a$ and $b$ are the remaining fit parameters, $N=298$ is the total number of $\mathrm{H}$ II regions in the map, $T_{\mathrm{A}, i}$ is the GDIGS moment 0 map intensity averaged over H II region $i, r_{i}$ is the distance from the HII region, and $r_{\mathrm{PDR}, i}$ is the radius of the region as given in the WISE catalog. $a$ is always calculated such that the total flux is conserved, i.e., the total integrated intensity of the model map must be equal to the total integrated intensity of the moment 0 map; $a$ is therefore not truly a free parameter.

Our preliminary best-fit values are $a=0.281, b=2.31$, and $c=0.14$, which corresponds to a leaking fraction of roughly 0.26 for W43 and 0.23 for G29 assuming that the average H II region not associated with either complex has $f_{\text {esc }}=0.17$. We show the model map and the difference map between the model and the GDIGS data in Figure 5.4. Overall, the model provides a good fit to the data, as is evident by the strong correlation between model and data (Pearson's $r=0.92$ ). Although the model still needs to be fine-tuned, it gives a valid representation of the data with the assumptions given above.

In $\S 3$, we show that the diffuse RRL emission near W43 is concentrated near two velocities (45 and $100 \mathrm{~km} \mathrm{~s}^{-1}$ ). While the $100 \mathrm{~km} \mathrm{~s}^{-1}$ component appears to be spatially related with W43 at a distance of $\sim 6 \mathrm{kpc}$, the origin of the $45 \mathrm{~km} \mathrm{~s}^{-1}$ component is less clear. The two velocity components are moderately correlated in $(\ell, b)$-space. With GDIGS, we can better untangle these two velocity components and possibly determine their origin. Shown in Figure 5.5 is the longitude-velocity diagram of the GDIGS data near W43. In the WIM-only diagram the two components are clearly separated in velocity. The absence of an interaction signature in velocity space may suggest that the 45 and $100 \mathrm{~km} \mathrm{~s}^{-1}$ gas are located at two distinct distances from the Sun, rather than being due to interacting gas clouds at a single distance.

Finally, we can use our GDIGS data of W43 to constrain the hardness of the 

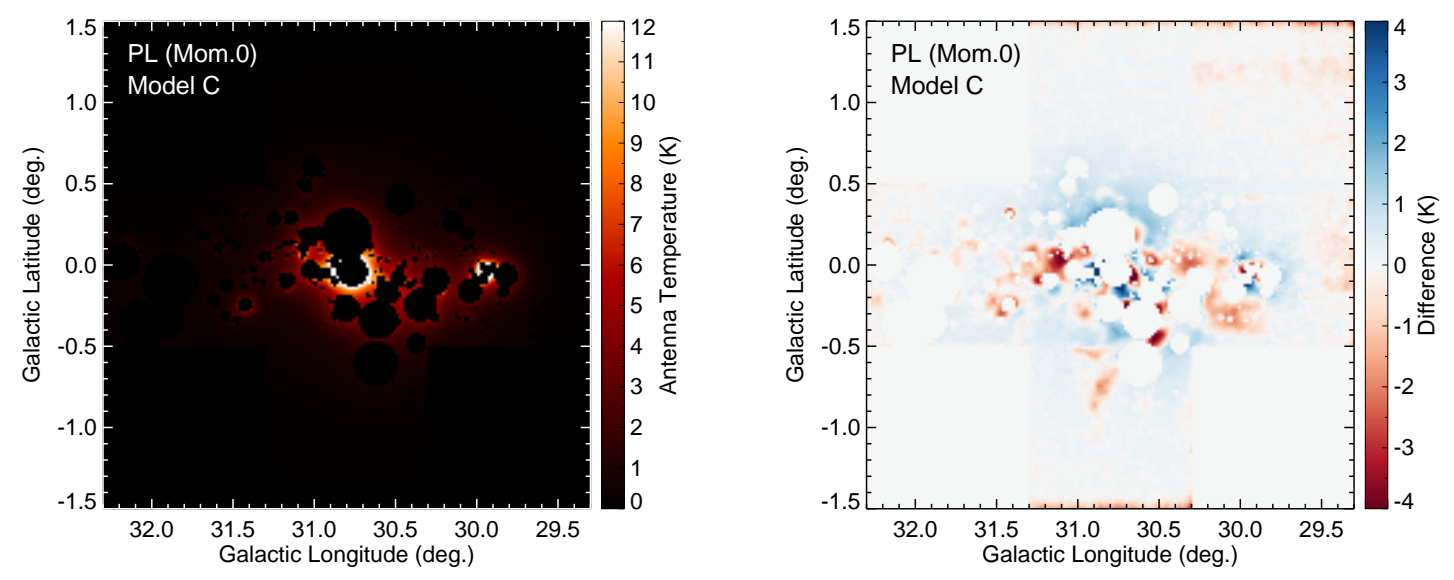

Figure 5.4: Left: model of diffuse hydrogen RRL emission, assuming a power-law decrease in RRL intensity with distance from each H II region and leaving the escape fraction as a free parameter. Right: difference map between the GDIGS moment 0 data and the model.

radiation field by deriving the $y^{+}=N\left({ }^{4} \mathrm{He}^{+}\right) / N\left(\mathrm{H}^{+}\right)$ionic abundance ratio. We perform this analysis individually for each $\mathrm{H}$ II region in the mapped range that has detectable helium emission and for the WIM. Our preliminary analysis shows that the average $\mathrm{H}$ II region has $y^{+}=0.102$, while the abundance ratio for the WIM is only $y^{+}=0.064$. This supports the assumption that the WIM is ionized by a softer radiation field than discrete $\mathrm{H}$ II regions. 

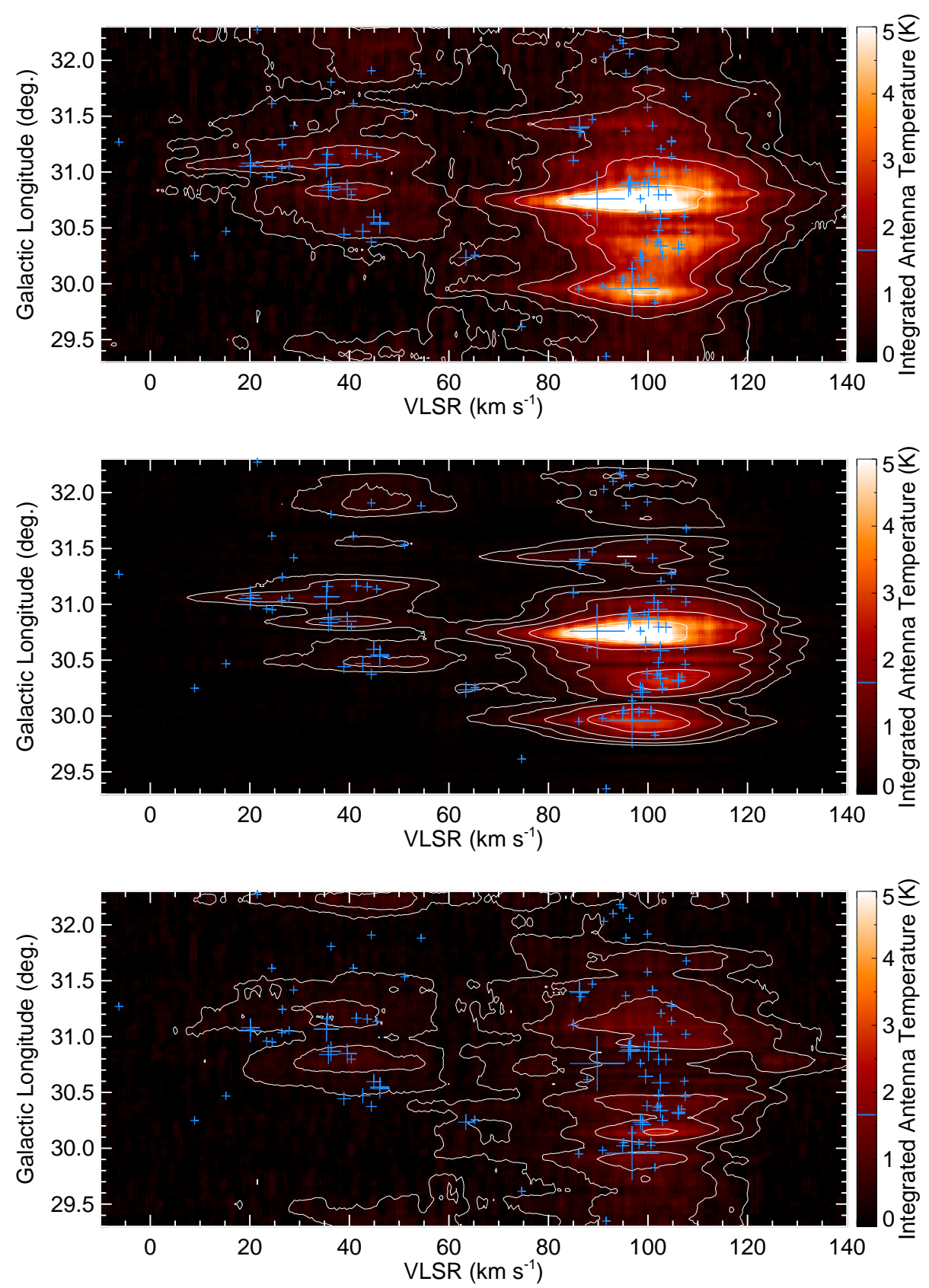

Figure 5.5: Top: longitude-velocity diagram of the W43 complex. The RRL emission clearly originates from two distinct velocities $\left(\sim 45\right.$ and $\left.\sim 100 \mathrm{~km} \mathrm{~s}^{-1}\right)$, with little emission in between. The $\sim 45 \mathrm{~km} \mathrm{~s}^{-1}$ emission is restricted to $\ell>30.3^{\circ}$, while the $\sim 100 \mathrm{~km} \mathrm{~s}^{-1}$ emission is extended across the entire mapped longitude range, possibly tracing the Scutum arm (see $\S 3$ ). Contours are at $0.25,0.5,1,2$, and $4 \mathrm{~K}$. The blue crosses show the locations of discrete H II regions in $(\ell, v)$-space. Middle: same, for discrete H II regions only. Bottom: same, for WIM-only map. The distribution of diffuse gas shows a clear separation between the 45 and $100 \mathrm{~km} \mathrm{~s}^{-1}$ component. 


\section{Chapter 6}

\section{Conclusions}

We have observed Galactic H II regions and the WIM in radio continuum and RRL emission and analyzed the impact of ionizing radiation emitted by OB stars on the ISM. We show that the PDR morphology strongly affects the amount of radiation leaking from an H II region and suggest that compact H II regions may only affect the ambient ISM on small scales. Larger H II region complexes, however, likely have a much greater impact on the ionization of the WIM and we demonstrate that the WIM intensity is correlated with the locations of such H II region complexes. We also

show that the $N\left({ }^{4} \mathrm{He}^{+}\right) / N\left(\mathrm{H}^{+}\right)$ionic abundance ratio decreases with distance from H II regions, suggesting a suppression of He-ionizing photons.

Below we individually address the key questions presented in $§ 1.6$.

\subsection{Mechanisms of Ionizing Photon Escape}

How is radiation originating from the O-stars within Galactic $\mathrm{H}$ II regions able to leak into the ISM?

In order to escape from an $\mathrm{H}$ II region, the radiation produced by the $\mathrm{OB}$ star must either leak through the surrounding PDR or the PDR must be clumpy, with low-density pathways in certain directions. In our study of the compact H II region NGC 7538 ( $(2)$, we produced a radio continuum map of the region and show that the ionized gas traced by the continuum emission is found predominantly toward the north and east of NGC 7538. This indicates that the fraction of leaking radiation is larger toward these directions. While the PDR is clearly visible in $12 \mu \mathrm{m}$ emission around the entire region, the gas column density toward the north-east is lower than in the south-west. We do not detect clear "holes" in the PDR as were found for other H II regions (e.g., for RCW 120; see Anderson et al., 2010). 
We also derive a dust temperature map of NGC7538 and find increased dust temperatures outside the HII region toward the north-east of NGC 7538. This suggests that radiation escaping in that direction heats the ambient medium, while the radiation is more confined toward directions with lower temperatures. Dust temperatures sharply drop farther east of NGC 7538, suggesting that a second PDR structure confines most of the radiation escaping from the primary PDR.

Our results from NGC 7538 compared with previous work (Anderson et al., 2010; Anderson et al., 2015) show that the PDR morphology strongly affects how radiation is able to leak from H II regions and into the ISM. In the case of NGC 7538, the radiation appears to primarily leak through a low-density PDR not showing any discontinuities, while for RCW 120 discrete, small holes in the PDR account for a significant fraction $(\sim 20 \%)$ of all leaked photons.

\subsection{Changes in the Radiation Field}

\section{How does the radiation field change as the photons escape through the $\mathrm{H}$ II region}

\section{PDR?}

The hardness of the radiation field can be estimated indirectly by the helium-tohydrogen $N\left({ }^{4} \mathrm{He}^{+}\right) / N\left(\mathrm{H}^{+}\right)$ionic abundance ratio since the first ionization potential of helium $(24.6 \mathrm{eV})$ is larger than that of hydrogen $(13.6 \mathrm{eV})$. For seven of the nine observed H II regions (one in $\S 2$ and six in $\S 4$ ), we measure a decrease in the ratio of integrated He-to-H RRL emission $y^{+}$with the distance from each region. This suggests that He-ionizing photons are selectively suppressed as radiation escapes from an H II region.

This work marks the first time that this effect has been observed in a larger (> 5 sources) sample of Galactic H II regions of different sizes, ages, and morphologies. Our results are consistent with previous observations (e.g., Pankonin et al., 1980) of individual H II regions and photoionization models (e.g., Wood \& Mathis, 2004; Weber et al., 2019).

We show that $y^{+}$decreases relatively steadily with the angular offset from most 
H II regions, regardless of the location of their PDR. This may support the hypothesis that a large fraction of He-ionizing photons is being absorbed within the H II region boundary, possibly due to their larger ionization cross-sections when compared to hydrogen. It is not fully clear why two H II regions (S206 and M17) do not follow the trend of decreasing $y^{+}$with distance from the region $(\S 4)$. For S206, $y^{+}$increases with the distance and for M17 the results are inconclusive, possibly because both regions have very hard radiation fields and little neutral helium within their hydrogen ionization fronts (Balser, 2006).

\subsection{The Impact of Leaking Photons on the ISM}

What fraction of ionizing radiation leaks from individual $\mathrm{H} \mathrm{II}$ regions and how does this radiation affect the ambient ISM?

We find a leaking fraction of $15 \pm 5 \%$ of the radio continuum emission for the compact Galactic H II region NGC $7538(\S 2)$. This is in rough agreement with the leaking fraction (25 $\pm 10 \%$ ) found by Anderson et al. (2015) for RCW 120 based on $\mathrm{H} \alpha$ observations. We show, however, that the leaked radiation is constrained by a second, outer PDR and seems to only affect the local ambient medium. Our measurements of the giant star formation complex W43, on the other hand, indicate that the decrease in RRL intensity with distance is much less steep than for NGC 7538. This suggests that radiation escaping from W43 may have a larger effect on maintaining the ionization of the WIM.

Our analysis of hydrogen RRL detections around a sample of eight Galactic H II regions $(\S 4)$ supports the hypothesis that larger H II regions affect the ISM more strongly. We find that the HII region size, traced by the PDR boundary, is related to the total amount of escaping ionizing radiation, such that physically smaller H II regions show a steeper decrease in RRL intensity with increasing distance from the region centers. The PDRs surrounding large and luminous H II regions may generally be weaker than those surrounding more compact regions. Large, evolved H II regions are also more likely to be density-bounded rather than radiation-bounded and may 
therefore allow a larger fraction of ionizing radiation to escape.

We further show that diffuse $8 \mu \mathrm{m}$ emission from PAHs is correlated with RRL emission from the WIM (§3). This may suggest that the soft-UV radiation field responsible for the excitation of PAHs has the same origin as the Lyman-continuum radiation maintaining the WIM. Toward lines of sight with the strongest RRL intensities the $8 \mu \mathrm{m}$ emission flattens out. We hypothesize that at these locations the radiation field is energetic enough to partially destroy the PAH population.

\subsection{Large-Scale Properties of the WIM}

What are the large-scale spatial distribution, kinematics, and physical properties of the WIM?

We find that the WIM in the range $40^{\circ}>\ell>18^{\circ}$ is concentrated near two velocities: $45 \mathrm{~km} \mathrm{~s}^{-1}$ and $100 \mathrm{~km} \mathrm{~s}^{-1}(\S 3)$. Mapping these velocities to distances is challenging since the KDA is not easily resolved for the diffuse gas. Since the $100 \mathrm{~km} \mathrm{~s}^{-1}$ emission is strongly associated with the HII region complex W43 at a distance of $5.5_{-0.3}^{+0.4} \mathrm{kpc}$, we assume that the $100 \mathrm{~km} \mathrm{~s}^{-1}$ component is also at the near kinematic distance of $\sim 6 \mathrm{kpc}$. The origin of the $45 \mathrm{~km} \mathrm{~s}^{-1}$ emission is less clear. The total H II region ionization rate at $\sim 45 \mathrm{~km} \mathrm{~s}^{-1}$ is higher at the far kinematic distance and we find a larger fraction of molecular gas at the far distance. This may suggest that the $45 \mathrm{~km} \mathrm{~s}^{-1}$ WIM emission originates at the far kinematic distance of $\sim 12 \mathrm{kpc}$ as well. However, there is a strong spatial correlation between the 45 and $100 \mathrm{~km} \mathrm{~s}^{-1}$ emission near W43. At this location it may be possible that the two velocity components are caused by interacting gas clouds at the same (near) distance.

Both the 45 and $100 \mathrm{~km} \mathrm{~s}^{-1}$ WIM component are spatially concentrated near $\ell=31^{\circ}$ and $\ell=24^{\circ}$. This comes as no surprise as the two largest and most luminous star forming complexes in the surveyed range are located near these directions. We find no evidence of depletion cavities in $\mathrm{HI}$ and ${ }^{13} \mathrm{CO}$ emission toward regions of strong RRL emission from the WIM. This suggests that neutral gas may coexist with the WIM in such regions. 
Through this thesis we have obtained a clearer picture of the connection between H II regions and the ISM. We analyzed the mechanisms that allow photons emitted by OB stars to escape from H II regions, showed that the radiation field softens as it escapes, estimated the effect this radiation has on the ISM, and constrained the large-scale distribution of the WIM. Together with our ongoing GDIGS observations, this work gives us a better understanding of the WIM and the impact of leaking radiation on the ISM from small to large spatial scales. 
Appendix A

Supplemental Data for Ionization Profiles of Galactic H II Regions

Table A.1: Observed Positions

\begin{tabular}{lccccl}
\hline Source & $\begin{array}{c}\text { RA } \\
(\mathrm{J} 2000)\end{array}$ & $\begin{array}{c}\text { Dec } \\
(\mathrm{J} 2000)\end{array}$ & $\begin{array}{c}\ell \\
(\text { deg. })\end{array}$ & $\begin{array}{c}b \\
(\text { deg. })\end{array}$ & PDR $^{a}$ \\
\hline S206_C & $04: 03: 15.8$ & $51: 18: 54$ & 150.593 & -0.951 & in \\
S206_E0.5 & $04: 03: 20.1$ & $51: 16: 57$ & 150.623 & -0.968 & out \\
S206_E1 & $04: 03: 24.2$ & $51: 15: 02$ & 150.652 & -0.985 & out \\
S206_E2 & $04: 03: 32.8$ & $51: 11: 07$ & 150.712 & -1.019 & out \\
S206_E3 & $04: 03: 41.1$ & $51: 07: 15$ & 150.771 & -1.053 & out \\
S206_W0.5 & $04: 03: 11.5$ & $51: 20: 51$ & 150.563 & -0.934 & out \\
S206_W1 & $04: 03: 07.5$ & $51: 22: 46$ & 150.534 & -0.917 & out \\
S206_W2 & $04: 02: 58.8$ & $51: 26: 41$ & 150.474 & -0.883 & out \\
S206_W3 & $04: 02: 50.3$ & $51: 30: 33$ & 150.415 & -0.849 & out \\
S206_W4 & $04: 02: 41.9$ & $51: 34: 25$ & 150.356 & -0.815 & out \\
& & & & & \\
S104_C & $20: 17: 41.9$ & $36: 45: 31$ & 74.762 & 0.619 & in \\
S104_N0.5 & $20: 17: 34.0$ & $36: 46: 49$ & 74.765 & 0.653 & in \\
S104_N1 & $20: 17: 26.2$ & $36: 48: 09$ & 74.769 & 0.687 & on \\
S104_N1.5 & $20: 17: 18.3$ & $36: 49: 27$ & 74.772 & 0.721 & out \\
S104_S0.5 & $20: 17: 49.8$ & $36: 44: 14$ & 74.759 & 0.585 & in \\
S104_S1 & $20: 17: 57.7$ & $36: 42: 56$ & 74.756 & 0.551 & on \\
S104_S1.5 & $20: 18: 05.5$ & $36: 41: 35$ & 74.752 & 0.517 & out \\
S104_S2.5 & $20: 18: 21.1$ & $36: 38: 57$ & 74.745 & 0.449 & out \\
& & & & & \\
G45_C & $19: 14: 19.6$ & $11: 09: 01$ & 45.448 & 0.064 & in \\
G45_E0.5 & $19: 14: 22.6$ & $11: 10: 56$ & 45.482 & 0.068 & out \\
G45_E1 & $19: 14: 25.8$ & $11: 12: 49$ & 45.516 & 0.071 & out \\
G45_E2 & $19: 14: 31.8$ & $11: 16: 39$ & 45.584 & 0.079 & out \\
G45_E3 & $19: 14: 38.0$ & $11: 20: 28$ & 45.652 & 0.086 & out \\
G45_W0.5 & $19: 14: 16.6$ & $11: 07: 06$ & 45.414 & 0.060 & in \\
G45_W1 & $19: 14: 13.4$ & $11: 05: 12$ & 45.380 & 0.057 & out \\
\hline & & & Continued on next page
\end{tabular}


Table A.1 - Continued from previous page

\begin{tabular}{lccccl}
\hline Source & RA & $\begin{array}{c}\text { Dec } \\
\text { (J2000) }\end{array}$ & $\begin{array}{c}\ell \\
\text { (J2000) }\end{array}$ & $\begin{array}{c}b \\
\text { (deg.) }\end{array}$ & $\begin{array}{l}\text { PDR }^{a} \\
\text { (deg.) }\end{array}$ \\
\hline G45_W2 & $19: 14: 07.4$ & $11: 01: 22$ & 45.312 & 0.049 & out \\
G45_W3 & $19: 14: 01.2$ & $10: 57: 34$ & 45.244 & 0.042 & out \\
& & & & & \\
M16_C & $18: 18: 37.0$ & $-13: 43: 46$ & 17.001 & 0.869 & in \\
M16_N0.5 & $18: 18: 29.3$ & $-13: 42: 58$ & 16.998 & 0.903 & in \\
M16_N1 & $18: 18: 21.5$ & $-13: 42: 09$ & 16.995 & 0.937 & in \\
M16_N1.5 & $18: 18: 13.9$ & $-13: 41: 18$ & 16.993 & 0.971 & in \\
M16_N2.5 & $18: 17: 58.4$ & $-13: 39: 41$ & 16.987 & 1.039 & out \\
M16_N4.5 & $18: 17: 27.3$ & $-13: 36: 22$ & 16.976 & 1.176 & out \\
M16_N6.5 & $18: 16: 56.5$ & $-13: 33: 05$ & 16.965 & 1.312 & out \\
M16_N8.5 & $18: 16: 25.4$ & $-13: 29: 45$ & 16.954 & 1.449 & out \\
M16_N10.5 & $18: 15: 54.6$ & $-13: 26: 27$ & 16.943 & 1.585 & out \\
M16_S0.5 & $18: 18: 44.8$ & $-13: 44: 35$ & 17.004 & 0.835 & in \\
M16_S1 & $18: 18: 52.5$ & $-13: 45: 23$ & 17.007 & 0.801 & in \\
M16_S1.5 & $18: 19: 00.1$ & $-13: 46: 15$ & 17.009 & 0.767 & in \\
M16_S2.5 & $18: 19: 15.7$ & $-13: 47: 51$ & 17.015 & 0.699 & in \\
M16_S3.5 & $18: 19: 31.3$ & $-13: 49: 33$ & 17.020 & 0.630 & on \\
M16_S5.5 & $18: 20: 02.2$ & $-13: 52: 49$ & 17.031 & 0.494 & out \\
M16_S9.5 & $18: 21: 04.3$ & $-13: 59: 22$ & 17.053 & 0.221 & out \\
M16_S11.5 & $18: 21: 35.3$ & $-14: 02: 38$ & 17.064 & 0.085 & out \\
& & & & & \\
M17_C & $18: 20: 30.1$ & $-16: 10: 44$ & 15.057 & -0.689 & in \\
M17_E1 & $18: 20: 41.1$ & $-16: 07: 35$ & 15.124 & -0.703 & in \\
M17_E1.5 & $18: 20: 46.4$ & $-16: 05: 57$ & 15.158 & -0.709 & in \\
M17_E2 & $18: 20: 51.8$ & $-16: 04: 24$ & 15.191 & -0.716 & in \\
M17_E3 & $18: 21: 02.8$ & $-16: 01: 15$ & 15.258 & -0.730 & in \\
M17_E4 & $18: 21: 13.7$ & $-15: 58: 06$ & 15.325 & -0.744 & out \\
M17_E5 & $18: 21: 24.5$ & $-15: 54: 55$ & 15.392 & -0.757 & out \\
M17_E6 & $18: 21: 35.4$ & $-15: 51: 46$ & 15.459 & -0.771 & out \\
M17_E7 & $18: 21: 46.3$ & $-15: 48: 37$ & 15.526 & -0.785 & out \\
M17_E9 & $18: 22: 08.0$ & $-15: 42: 17$ & 15.660 & -0.812 & out \\
M17_E11 & $18: 22: 29.6$ & $-15: 35: 57$ & 15.794 & -0.839 & out \\
M17_W1 & $18: 20: 19.1$ & $-16: 13: 53$ & 14.990 & -0.675 & in \\
\hline & & & Continued on next page
\end{tabular}


Table A.1 - Continued from previous page

\begin{tabular}{lccccl}
\hline Source & RA & $\begin{array}{c}\text { Dec } \\
(\mathrm{J} 2000)\end{array}$ & $\begin{array}{c}\ell \\
(\mathrm{deg})\end{array}$ & $\begin{array}{c}b \\
(\text { deg. })\end{array}$ & $\mathrm{PDR}^{a}$ \\
\hline M17_W1.5 & $18: 20: 13.8$ & $-16: 15: 31$ & 14.956 & -0.669 & on \\
M17_W2 & $18: 20: 08.3$ & $-16: 17: 03$ & 14.923 & -0.662 & out \\
M17_W3 & $18: 19: 57.3$ & $-16: 20: 12$ & 14.856 & -0.648 & out \\
M17_W4 & $18: 19: 46.3$ & $-16: 23: 21$ & 14.789 & -0.634 & out \\
M17_W5 & $18: 19: 35.6$ & $-16: 26: 32$ & 14.722 & -0.621 & out \\
& & & & & \\
Orion_C & $05: 35: 15.5$ & $-05: 23: 35$ & 209.012 & -19.389 & in \\
Orion_N1 & $05: 35: 28.6$ & $-05: 21: 06$ & 208.999 & -19.322 & in \\
Orion_N2 & $05: 35: 41.7$ & $-05: 18: 38$ & 208.986 & -19.255 & in \\
Orion_N3 & $05: 35: 54.7$ & $-05: 16: 09$ & 208.973 & -19.188 & on \\
Orion_N4 & $05: 36: 08.0$ & $-05: 13: 39$ & 208.960 & -19.120 & out \\
Orion_N5 & $05: 36: 21.1$ & $-05: 11: 10$ & 208.947 & -19.053 & out \\
Orion_S1 & $05: 35: 02.5$ & $-05: 26: 03$ & 209.025 & -19.456 & in \\
Orion_S2 & $05: 34: 49.4$ & $-05: 28: 32$ & 209.038 & -19.523 & in \\
Orion_S3 & $05: 34: 36.3$ & $-05: 31: 00$ & 209.051 & -19.590 & in \\
Orion_S4 & $05: 34: 23.0$ & $-05: 33: 30$ & 209.064 & -19.658 & in \\
Orion_S5 & $05: 34: 09.9$ & $-05: 35: 58$ & 209.077 & -19.725 & in \\
Orion_S6 & $05: 33: 56.9$ & $-05: 38: 26$ & 209.090 & -19.792 & in \\
Orion_S7 & $05: 33: 43.8$ & $-05: 40: 55$ & 209.103 & -19.859 & in \\
Orion_S9 & $05: 33: 17.6$ & $-05: 45: 51$ & 209.129 & -19.993 & out \\
Orion_S11 & $05: 32: 51.1$ & $-05: 50: 45$ & 209.154 & -20.128 & out \\
& & & & & \\
N49_C & $18: 44: 45.3$ & $-03: 45: 21$ & 28.828 & -0.228 & in \\
N49_E0.5 & $18: 44: 47.8$ & $-03: 43: 23$ & 28.862 & -0.222 & out \\
N49_E1 & $18: 44: 50.1$ & $-03: 41: 27$ & 28.895 & -0.216 & out \\
N49_E2 & $18: 44: 54.8$ & $-03: 37: 28$ & 28.963 & -0.203 & out \\
N49_W0.5 & $18: 44: 42.9$ & $-03: 47: 20$ & 28.794 & -0.234 & out \\
N49_W1 & $18: 44: 40.5$ & $-03: 49: 16$ & 28.761 & -0.240 & out \\
N49_W2 & $18: 44: 35.9$ & $-03: 53: 15$ & 28.693 & -0.253 & out \\
& & & & & \\
G29_C & $18: 46: 04.7$ & $-02: 39: 27$ & 29.956 & -0.020 & in \\
G29_N0.5 & $18: 45: 57.5$ & $-02: 38: 28$ & 29.957 & 0.014 & on \\
G29_N1 & $18: 45: 50.3$ & $-02: 37: 29$ & 29.958 & 0.048 & out \\
\hline & & & & Continued on next page
\end{tabular}


Table A.1 - Continued from previous page

\begin{tabular}{lccccl}
\hline Source & $\begin{array}{c}\text { RA } \\
(\text { J2000 })\end{array}$ & $\begin{array}{c}\text { Dec } \\
(\mathrm{J} 2000)\end{array}$ & $\begin{array}{c}\ell \\
(\text { deg. })\end{array}$ & $\begin{array}{c}b \\
(\text { deg. })\end{array}$ & PDR $^{a}$ \\
\hline G29_N1.5 & $18: 45: 43.0$ & $-02: 36: 28$ & 29.959 & 0.083 & out \\
G29_N2 & $18: 45: 35.8$ & $-02: 35: 29$ & 29.960 & 0.117 & out \\
G29_N3 & $18: 45: 21.5$ & $-02: 33: 31$ & 29.962 & 0.185 & out \\
G29_N4 & $18: 45: 07.2$ & $-02: 31: 32$ & 29.964 & 0.253 & out \\
G29_N5 & $18: 44: 52.6$ & $-02: 29: 33$ & 29.966 & 0.322 & out \\
G29_S0.5 & $18: 46: 11.8$ & $-02: 40: 26$ & 29.955 & -0.054 & in \\
G29_S1 & $18: 46: 19.0$ & $-02: 41: 25$ & 29.954 & -0.088 & in \\
G29_S1.5 & $18: 46: 26.3$ & $-02: 42: 26$ & 29.953 & -0.123 & on \\
G29_S2 & $18: 46: 33.5$ & $-02: 43: 25$ & 29.952 & -0.157 & out \\
G29_S3 & $18: 46: 47.8$ & $-02: 45: 23$ & 29.950 & -0.225 & out \\
G29_S4 & $18: 47: 02.1$ & $-02: 47: 21$ & 29.948 & -0.293 & out \\
G29_S5 & $18: 47: 16.7$ & $-02: 49: 21$ & 29.946 & -0.362 & out \\
\hline
\end{tabular}

Note — a "out"... outside the PDR, "on" ... on the PDR, "in"... inside the PDR. 


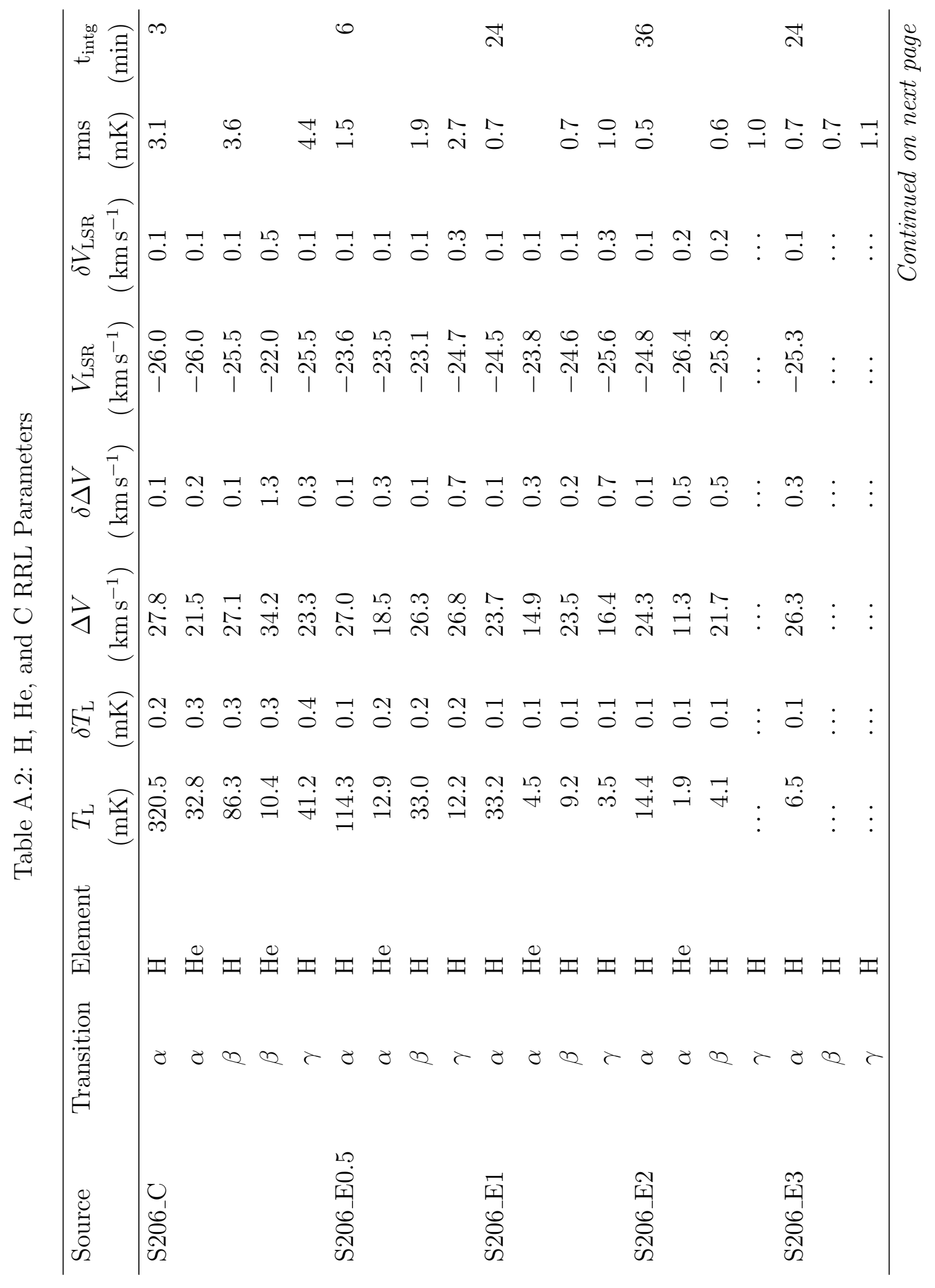




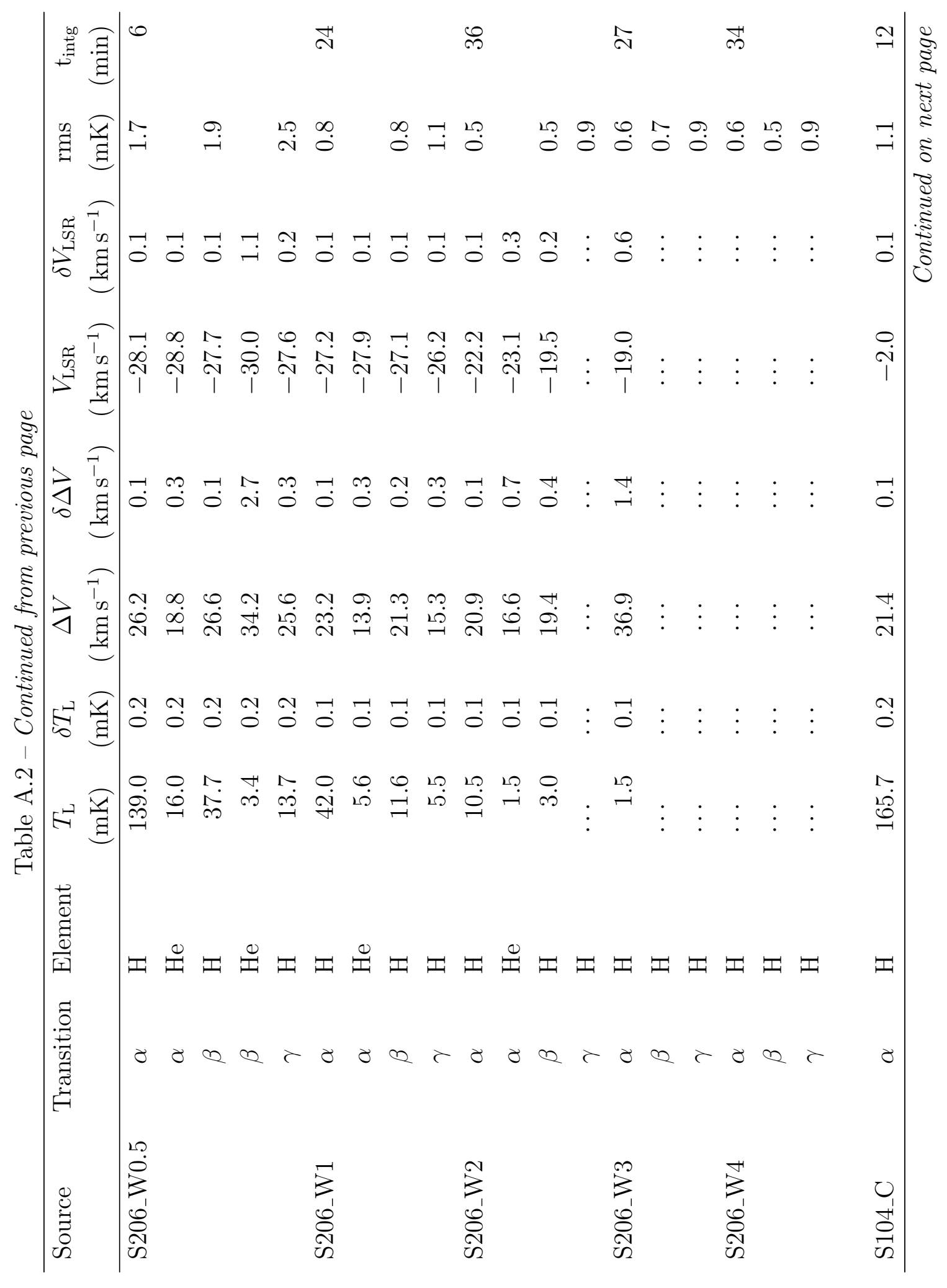




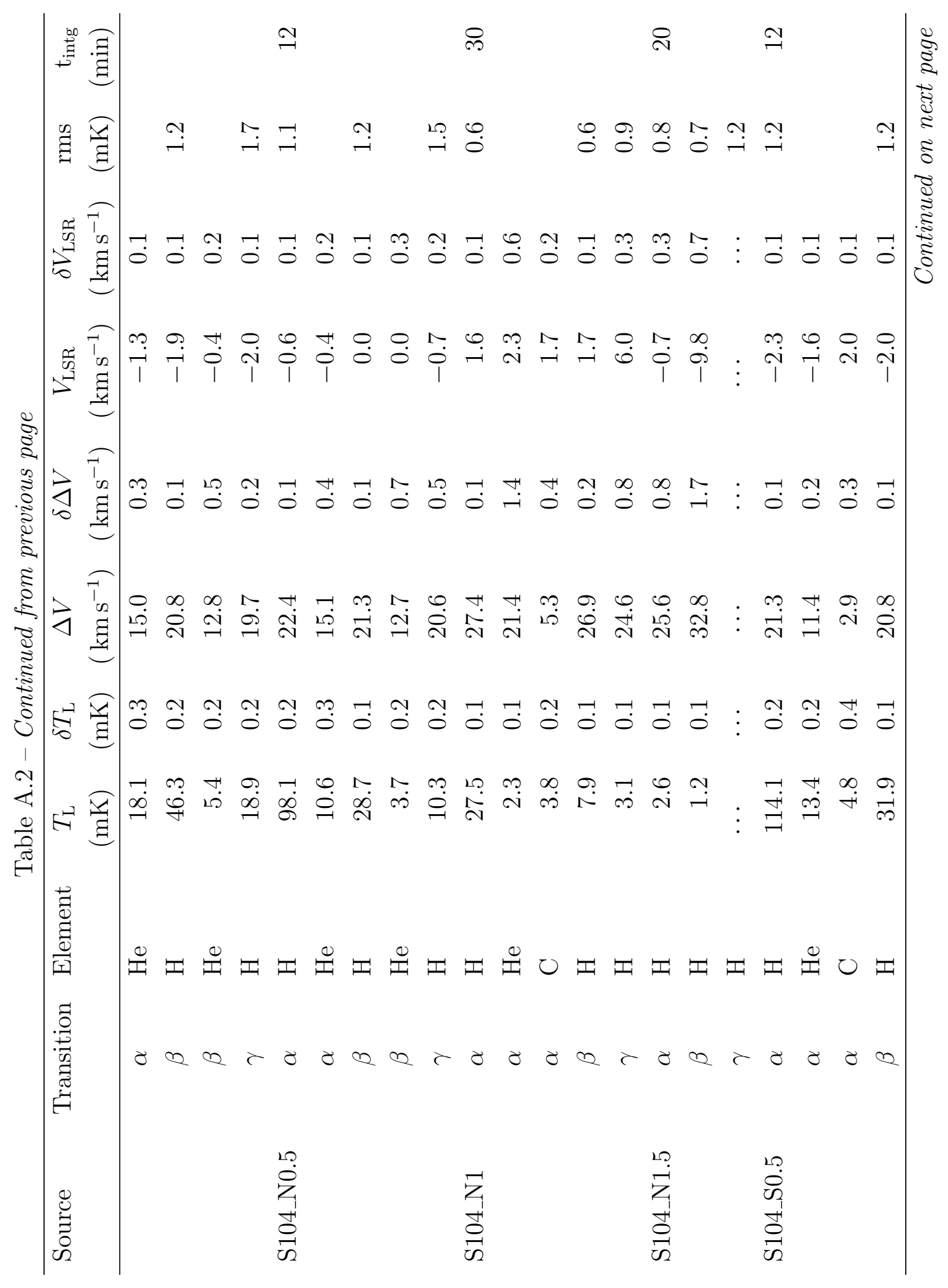




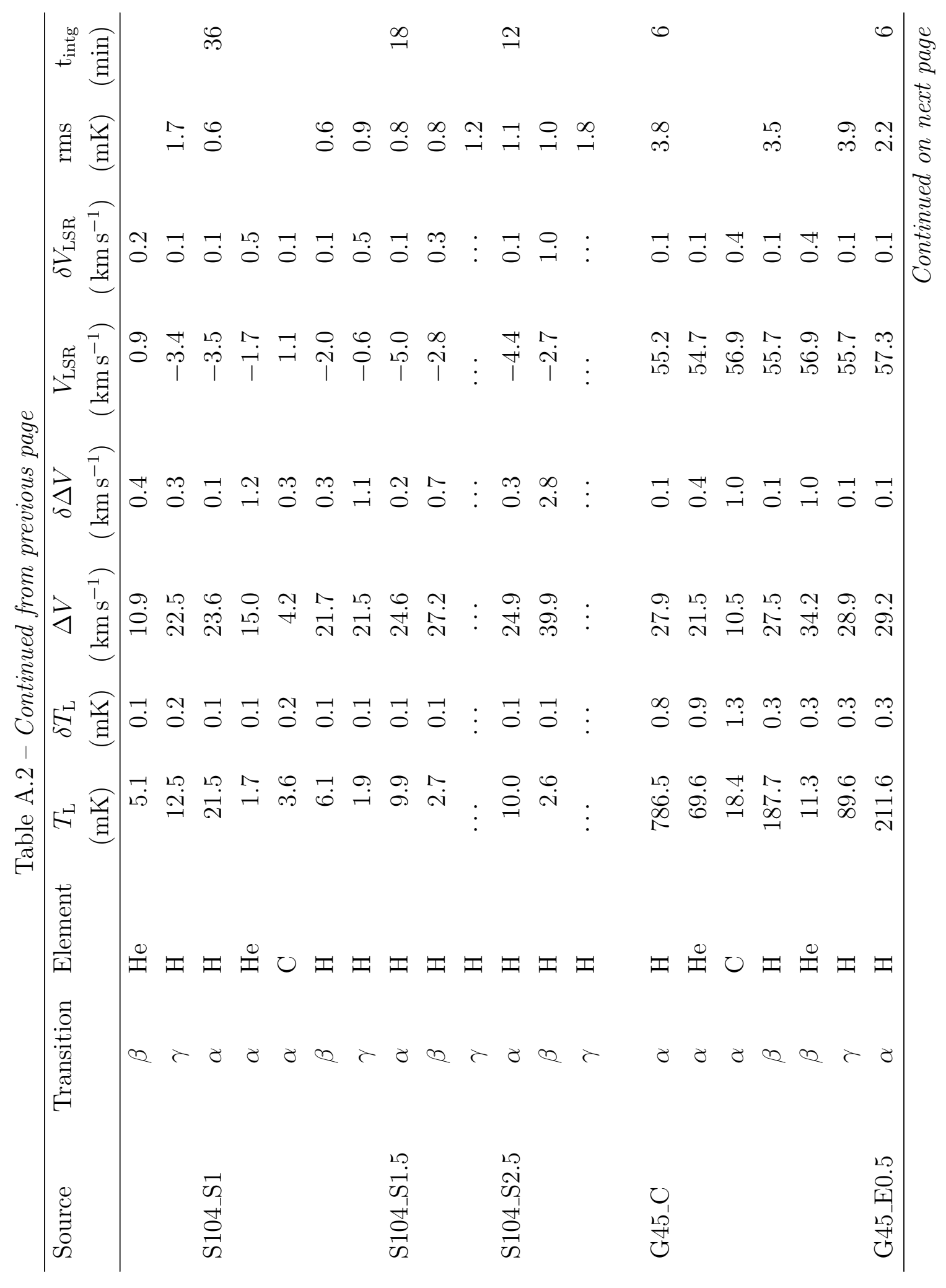




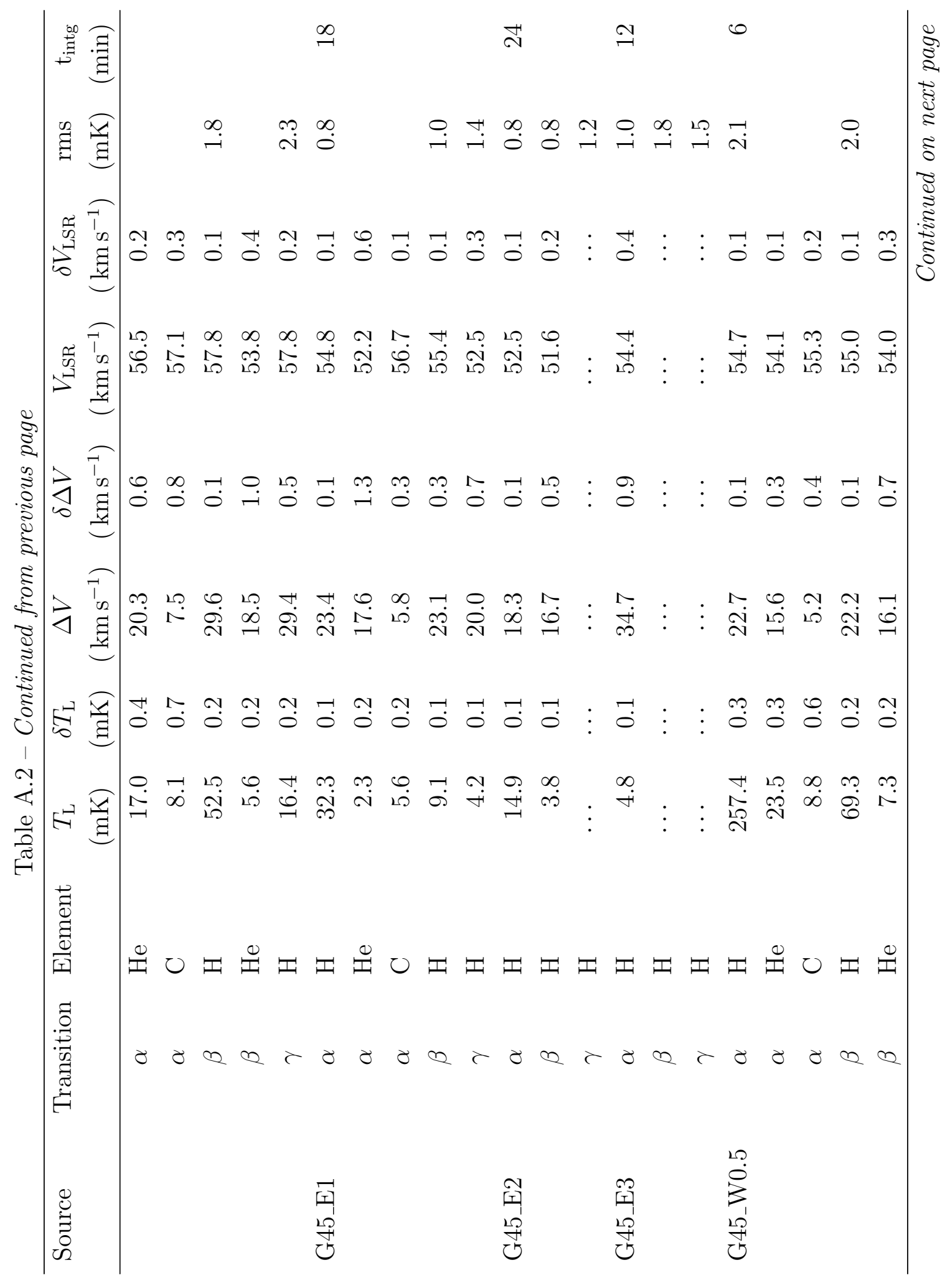




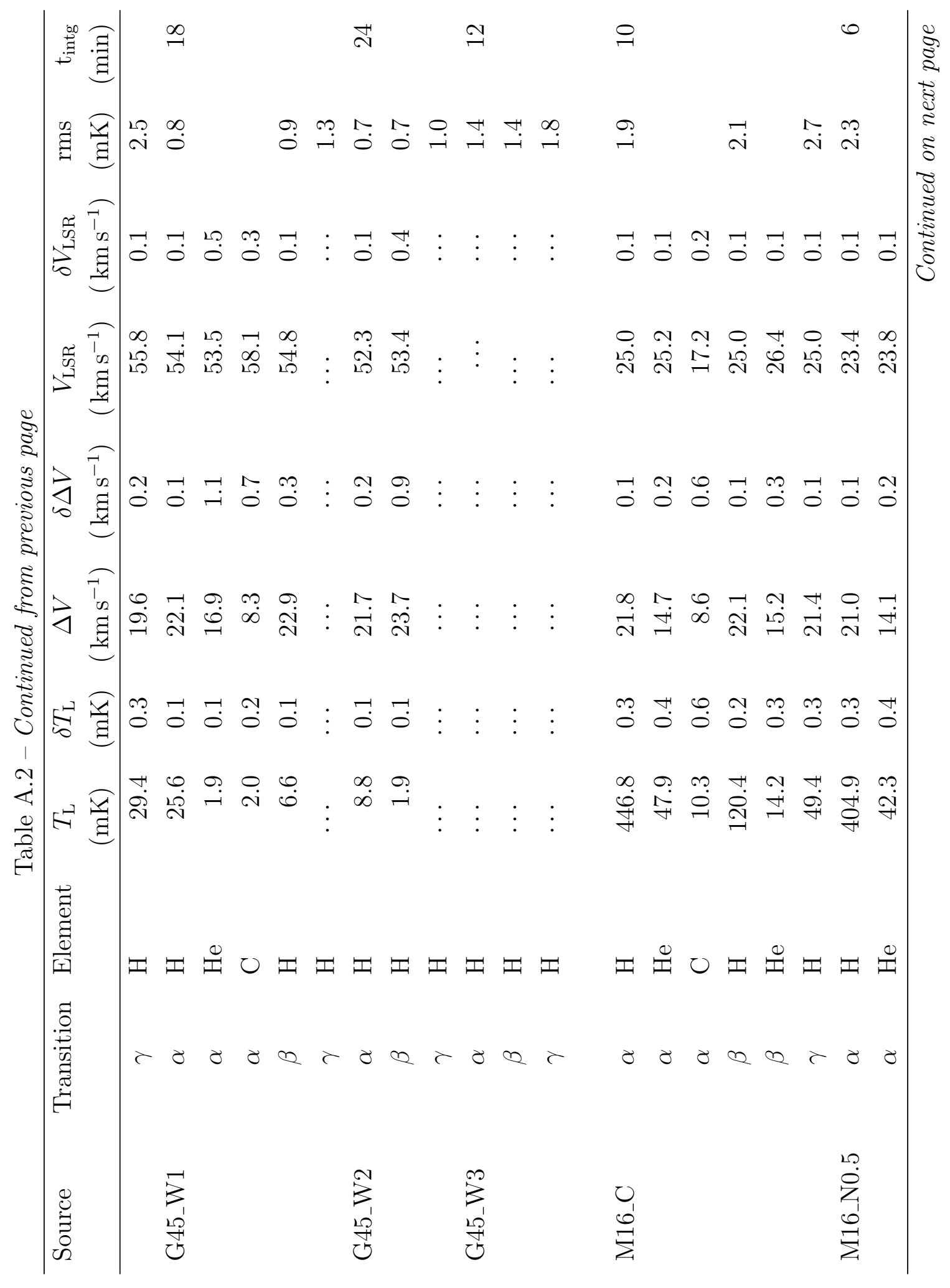




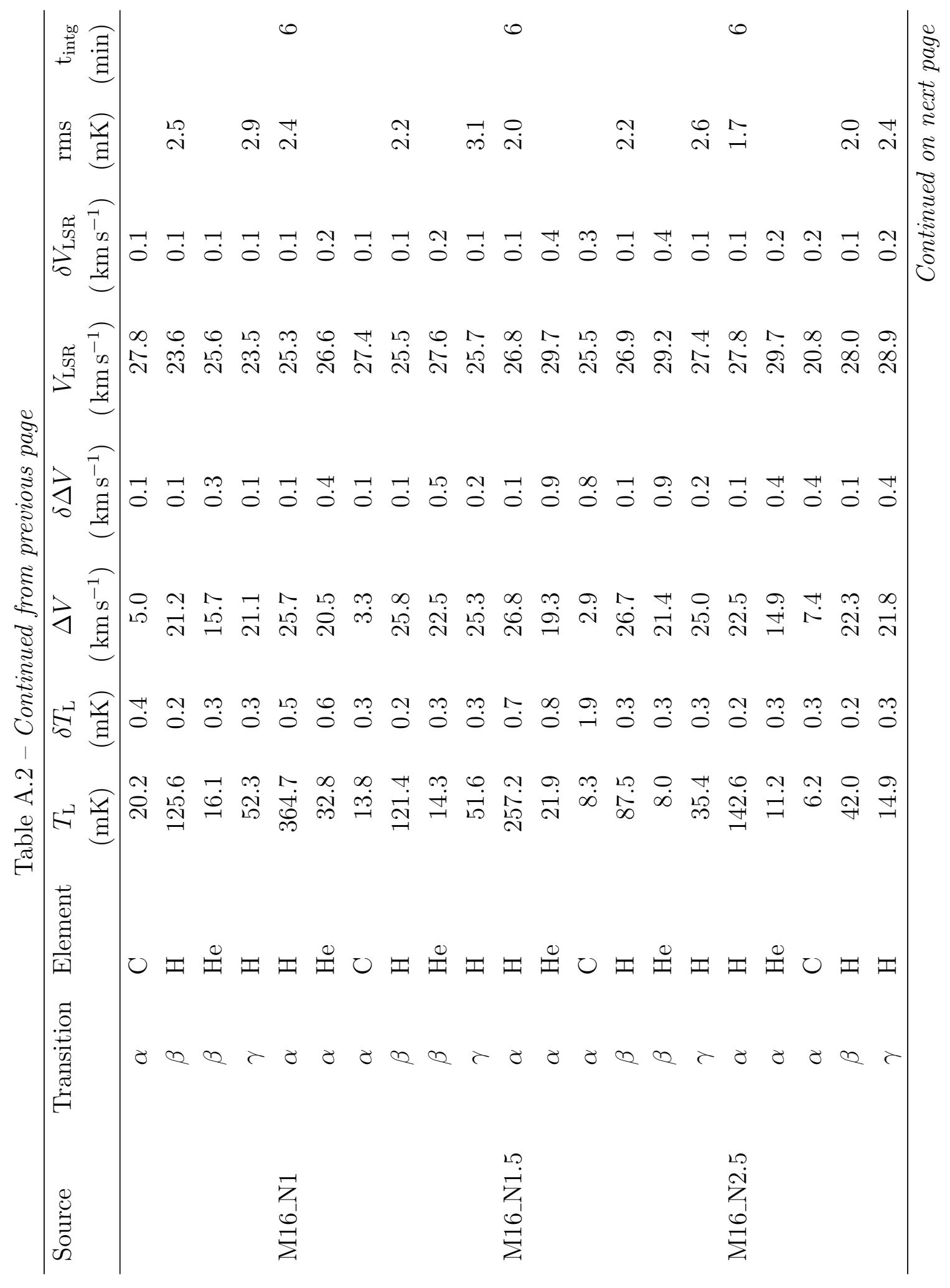




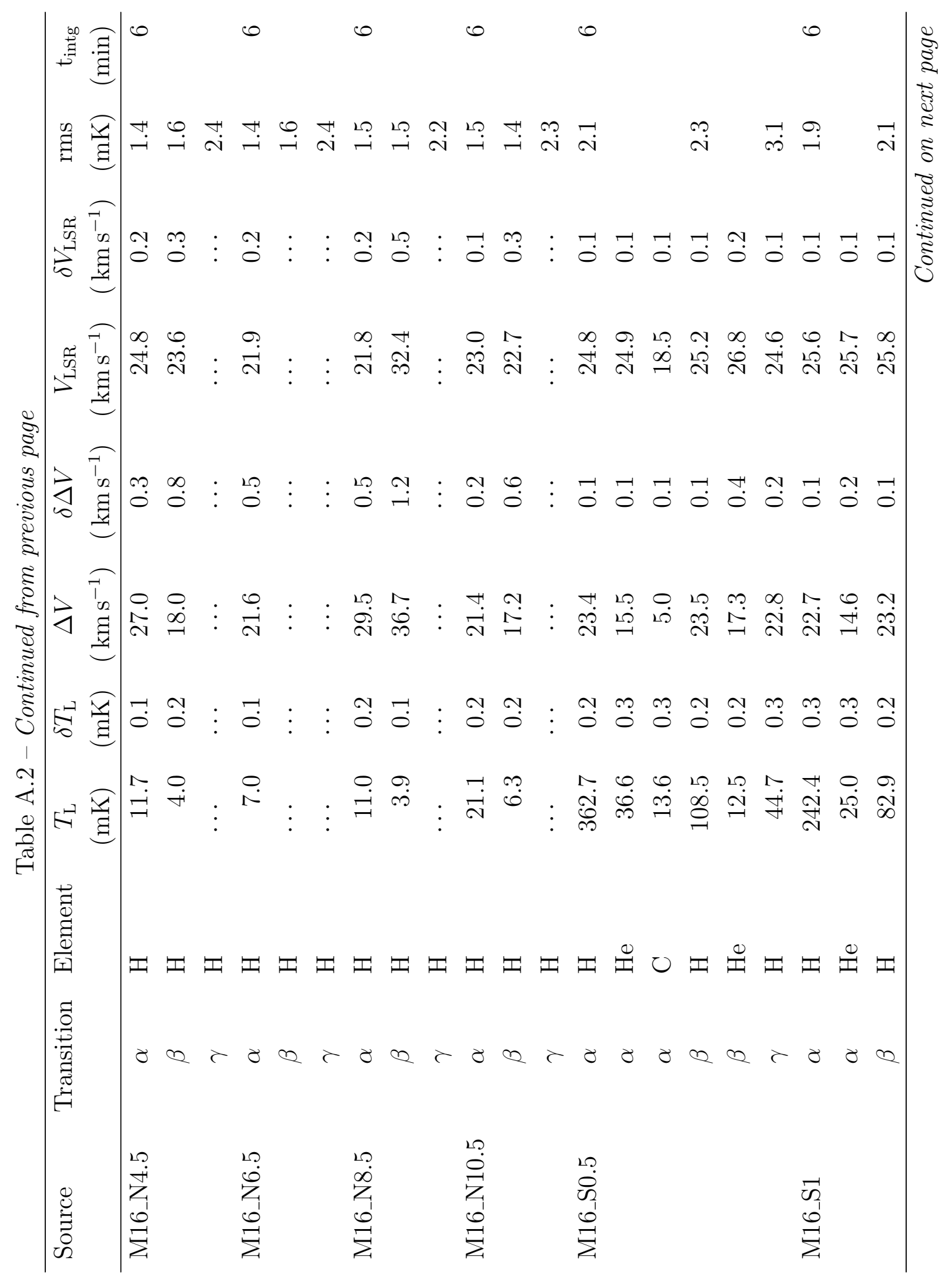




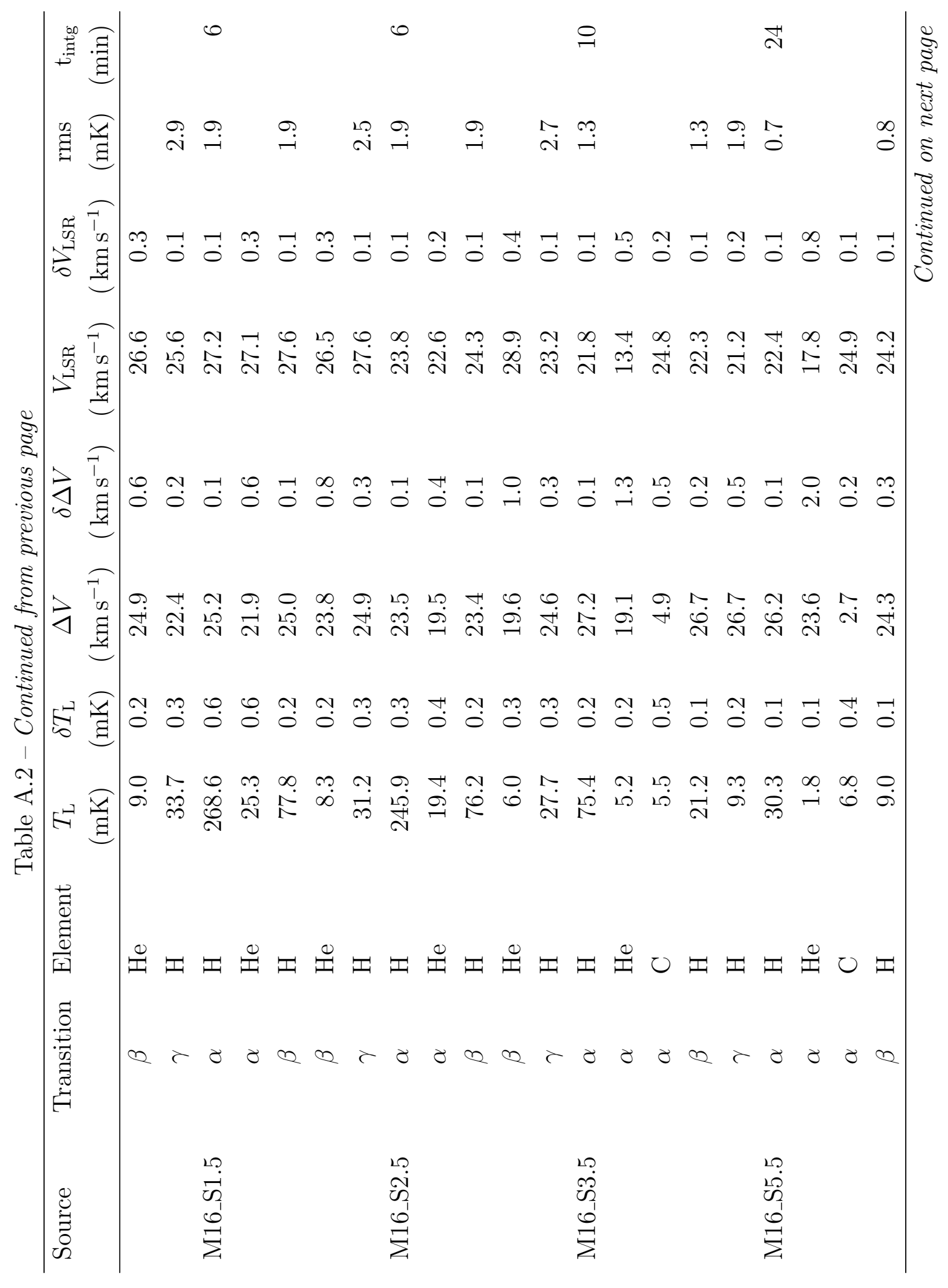




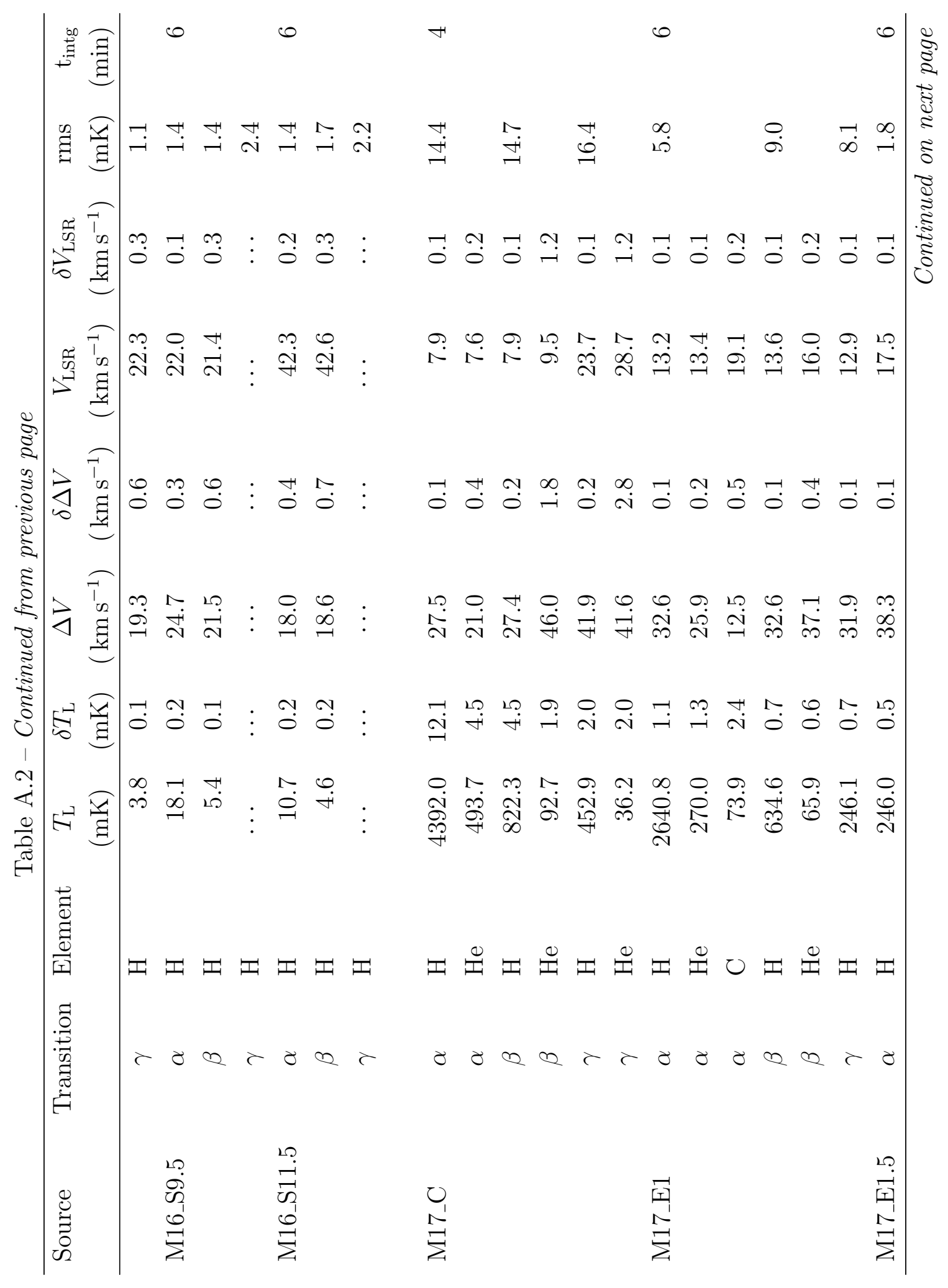




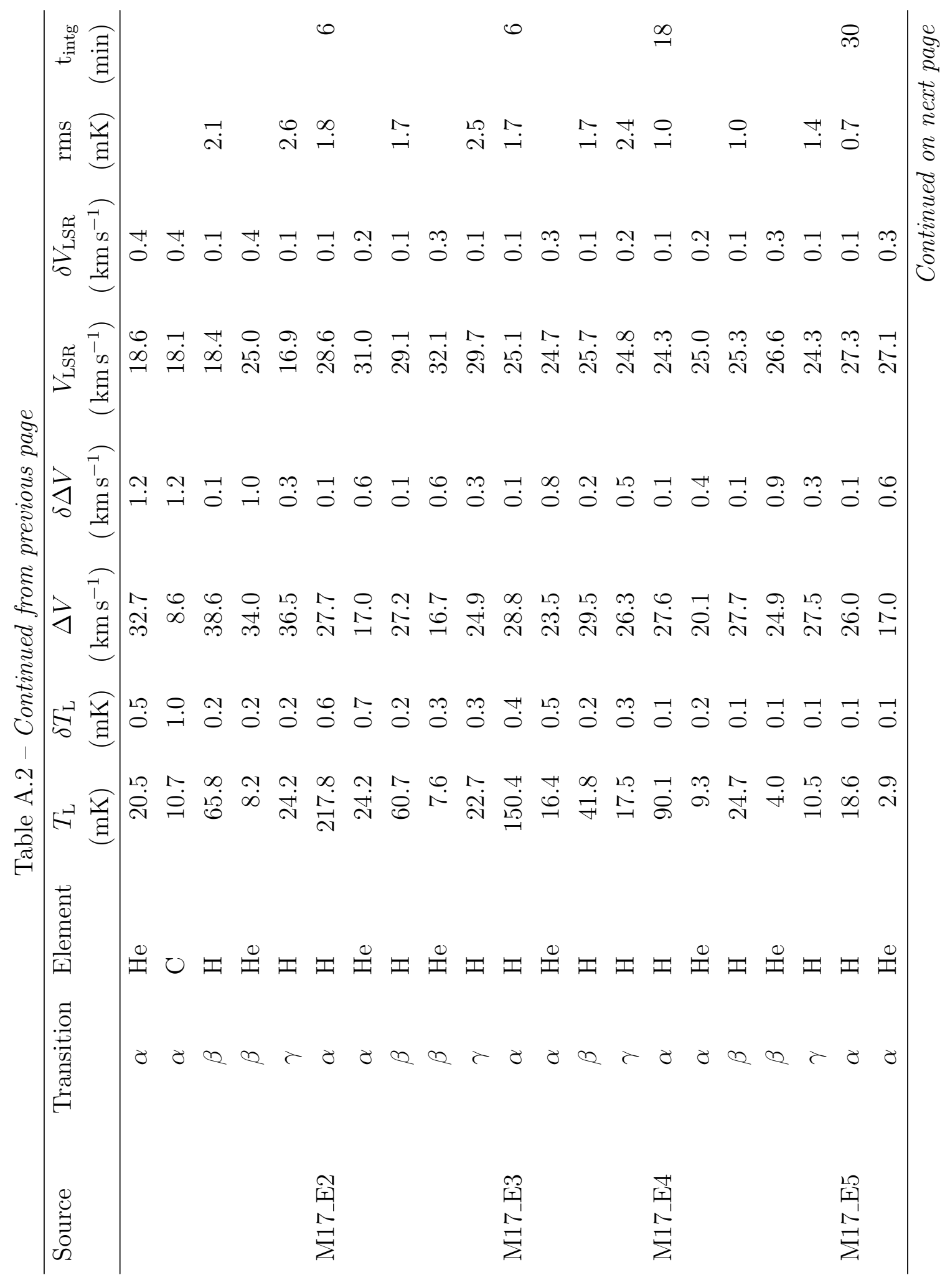




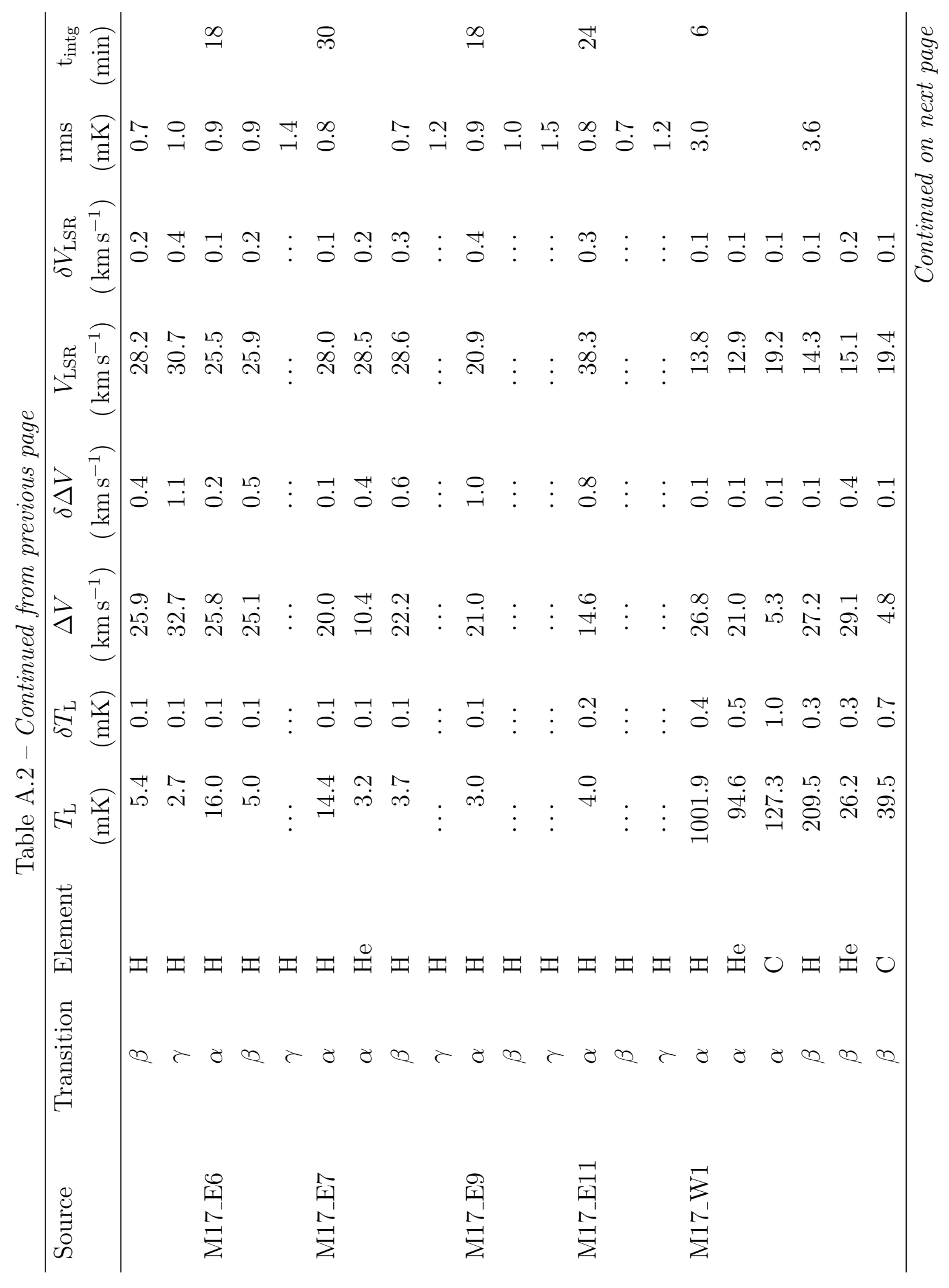




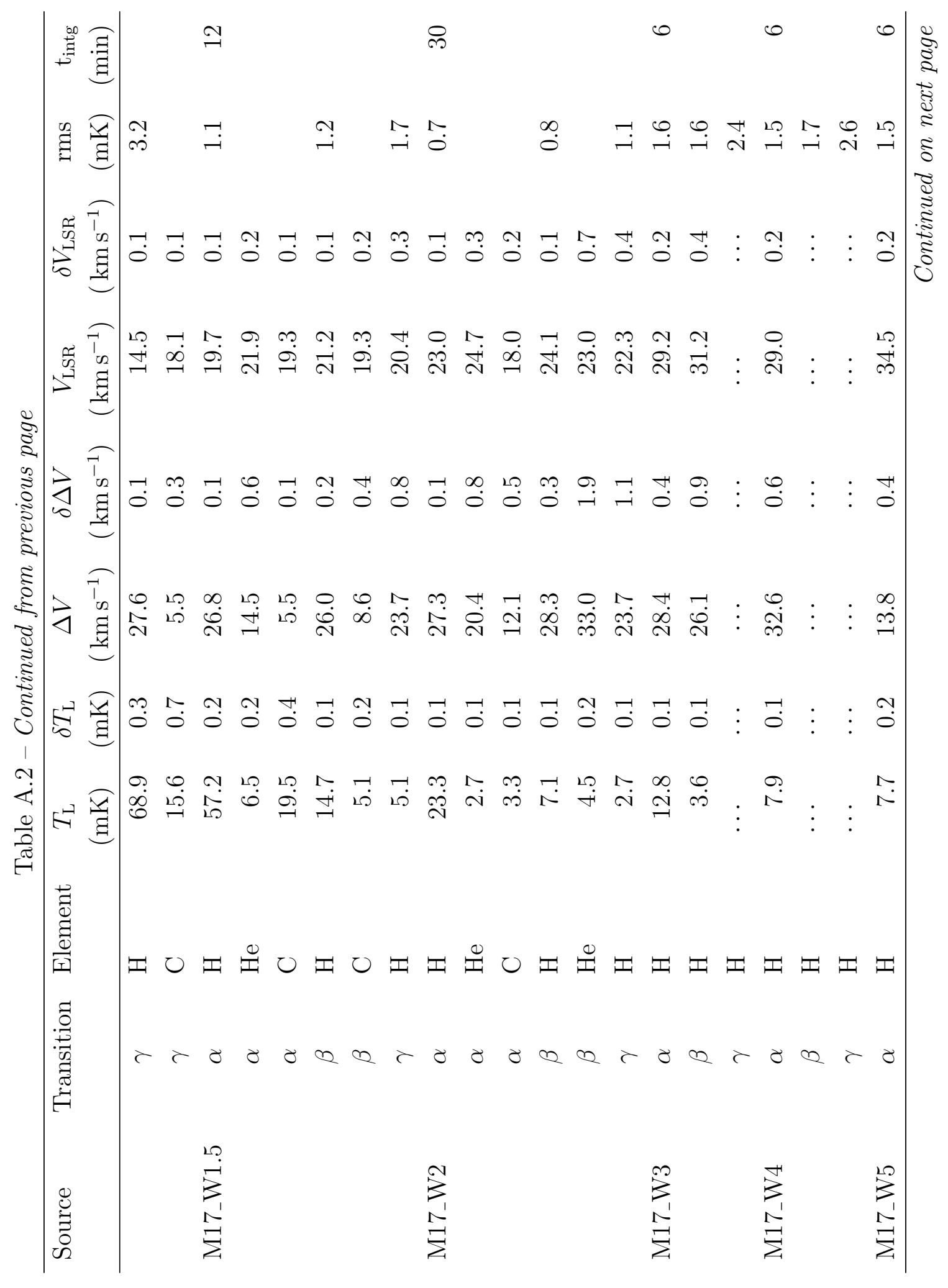




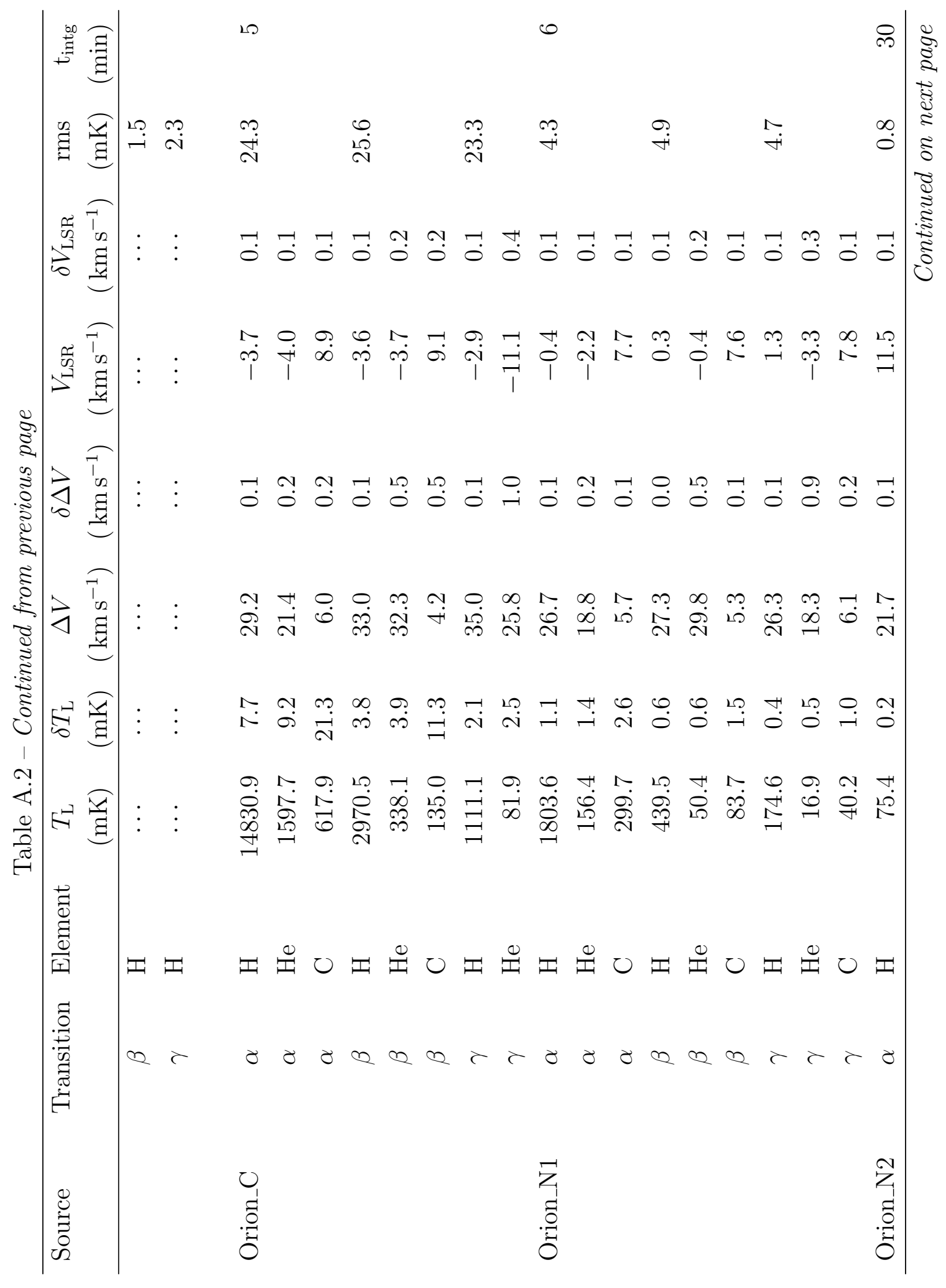




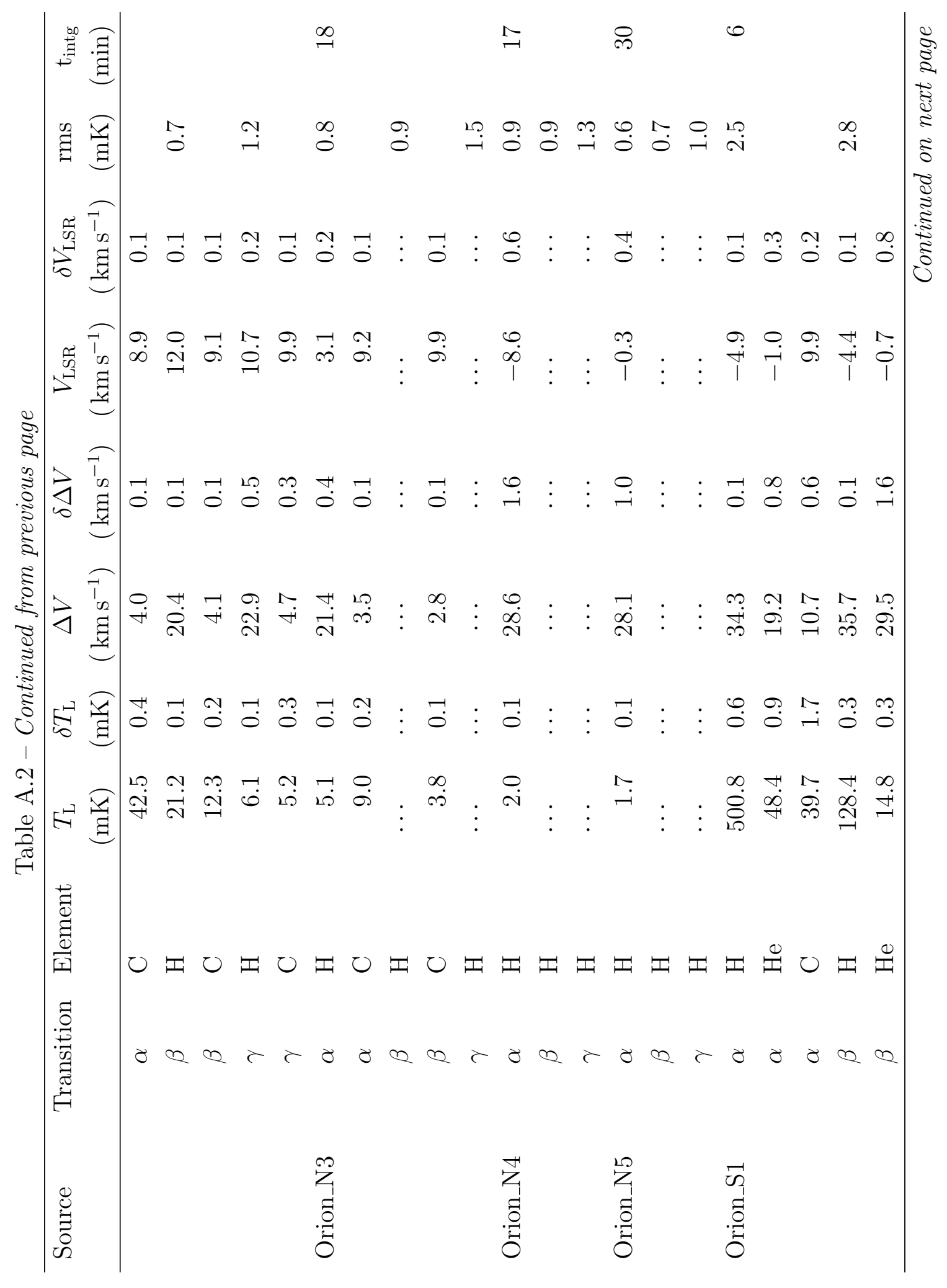




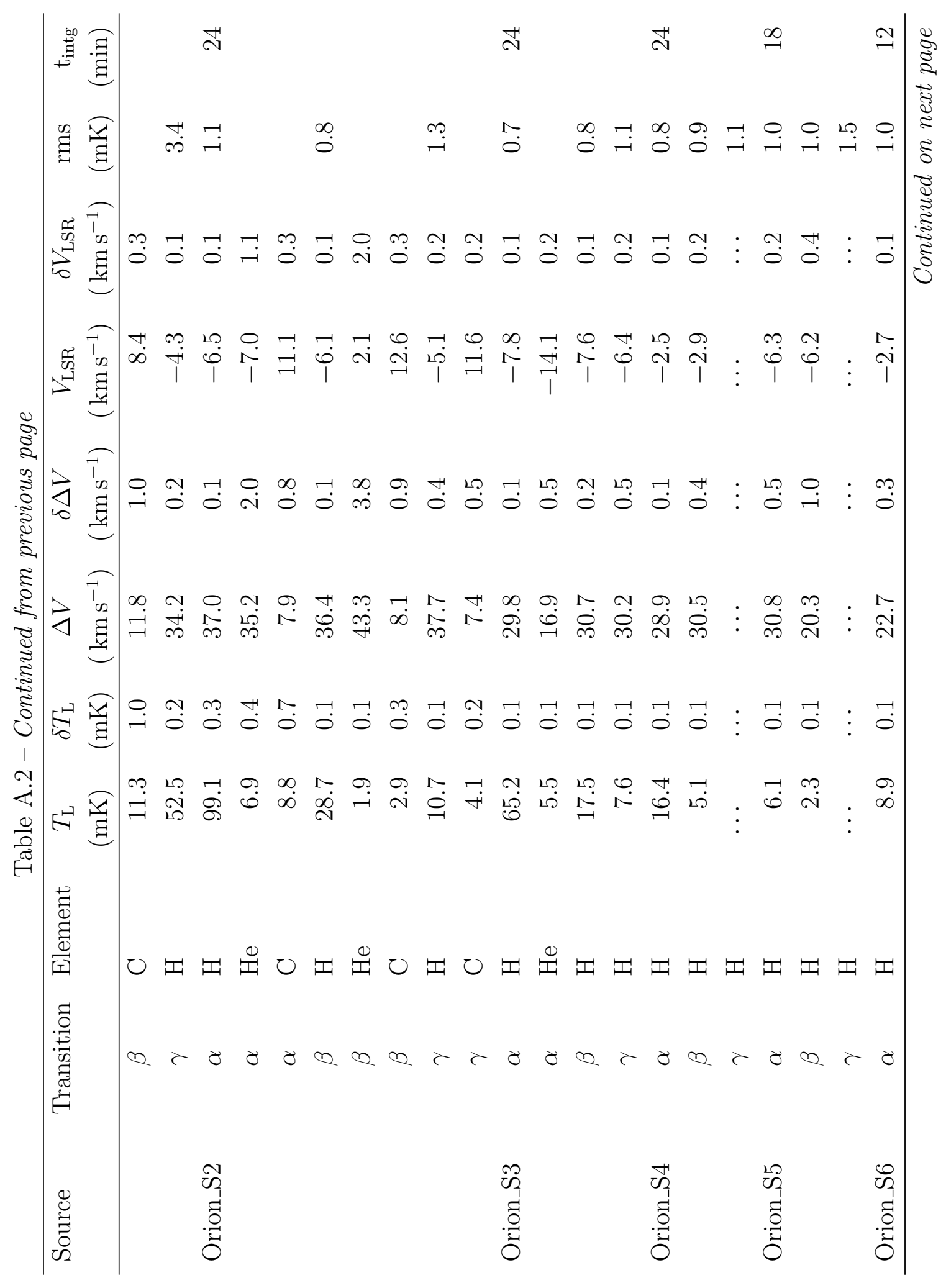




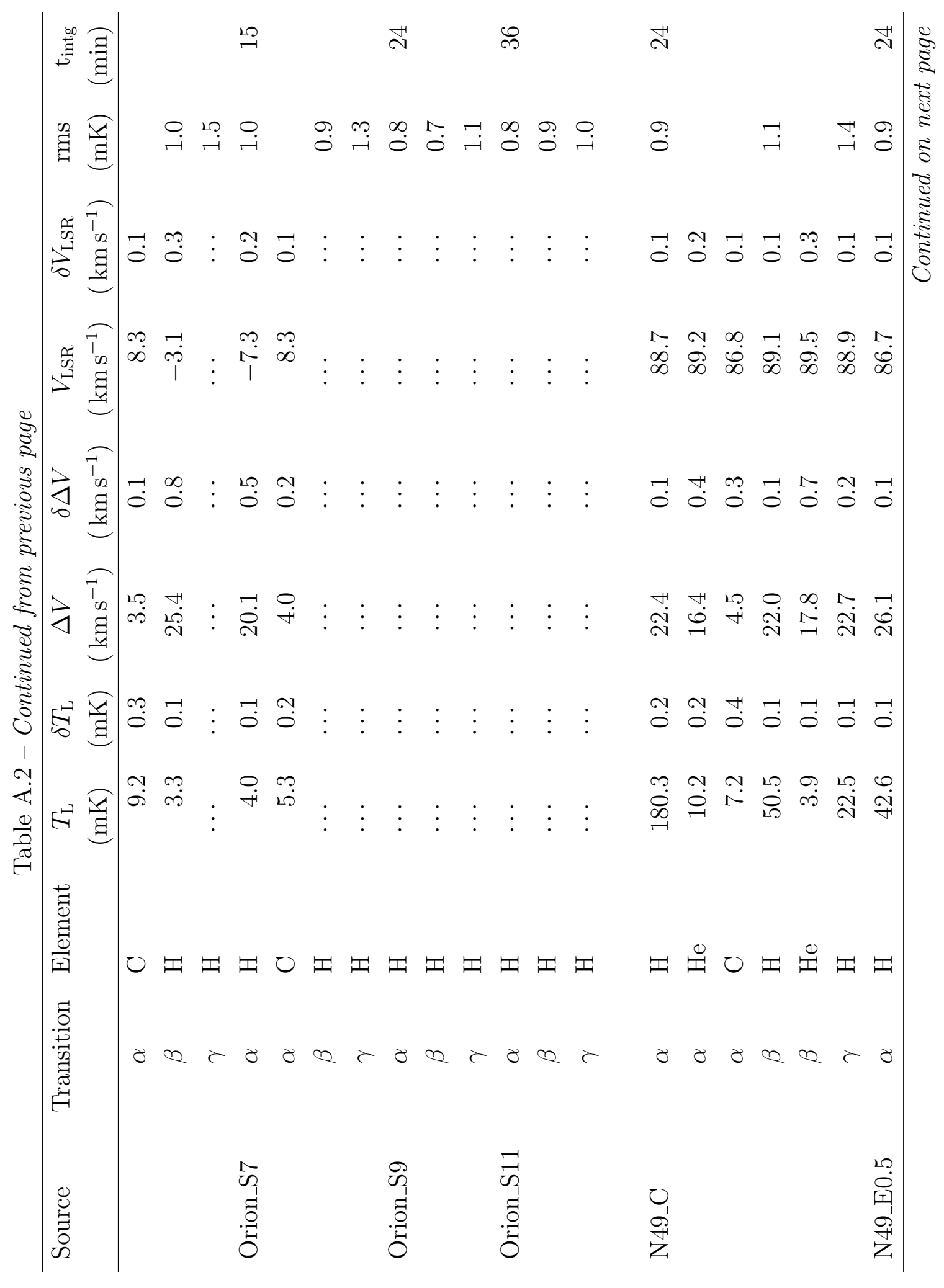




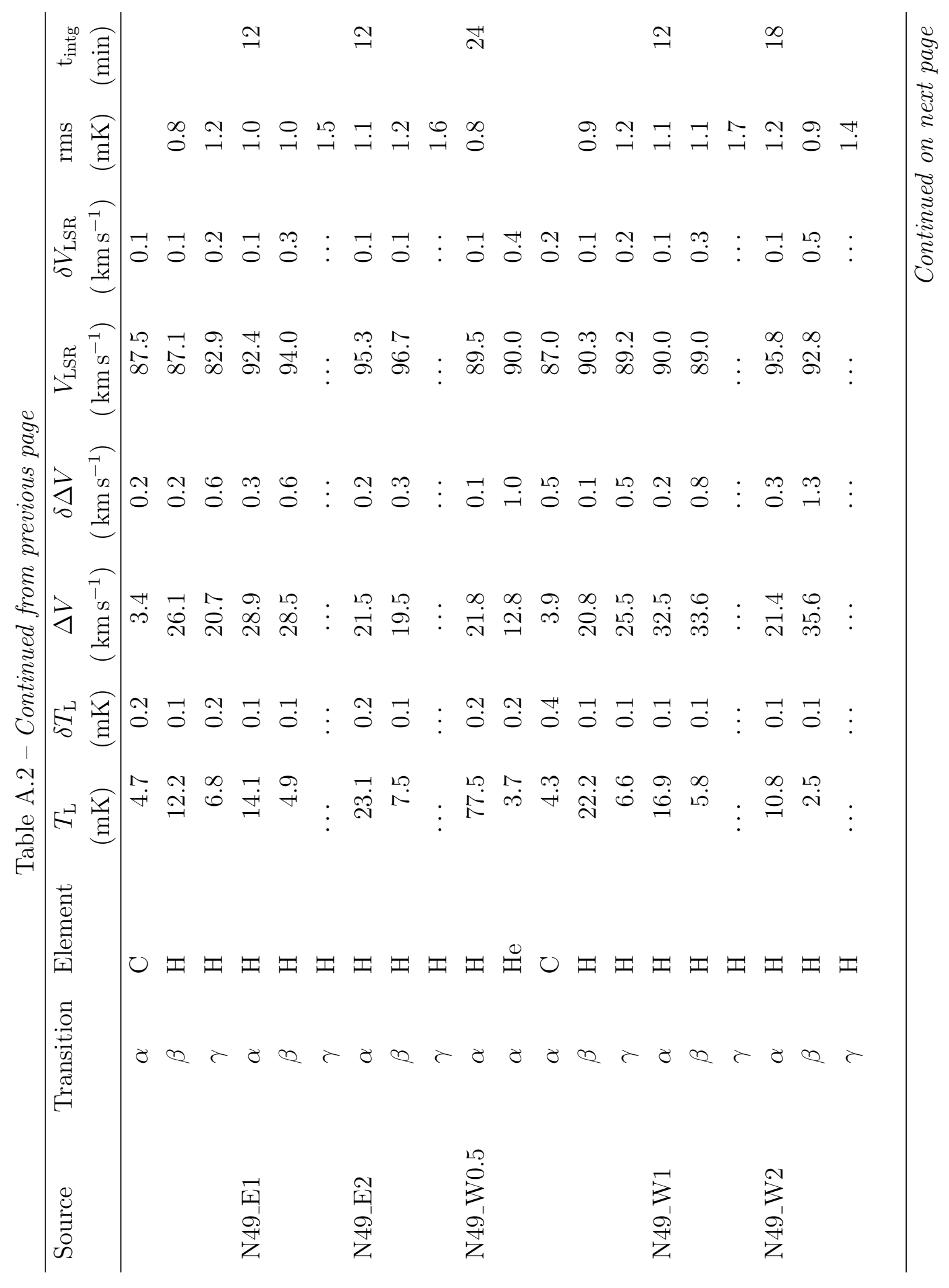




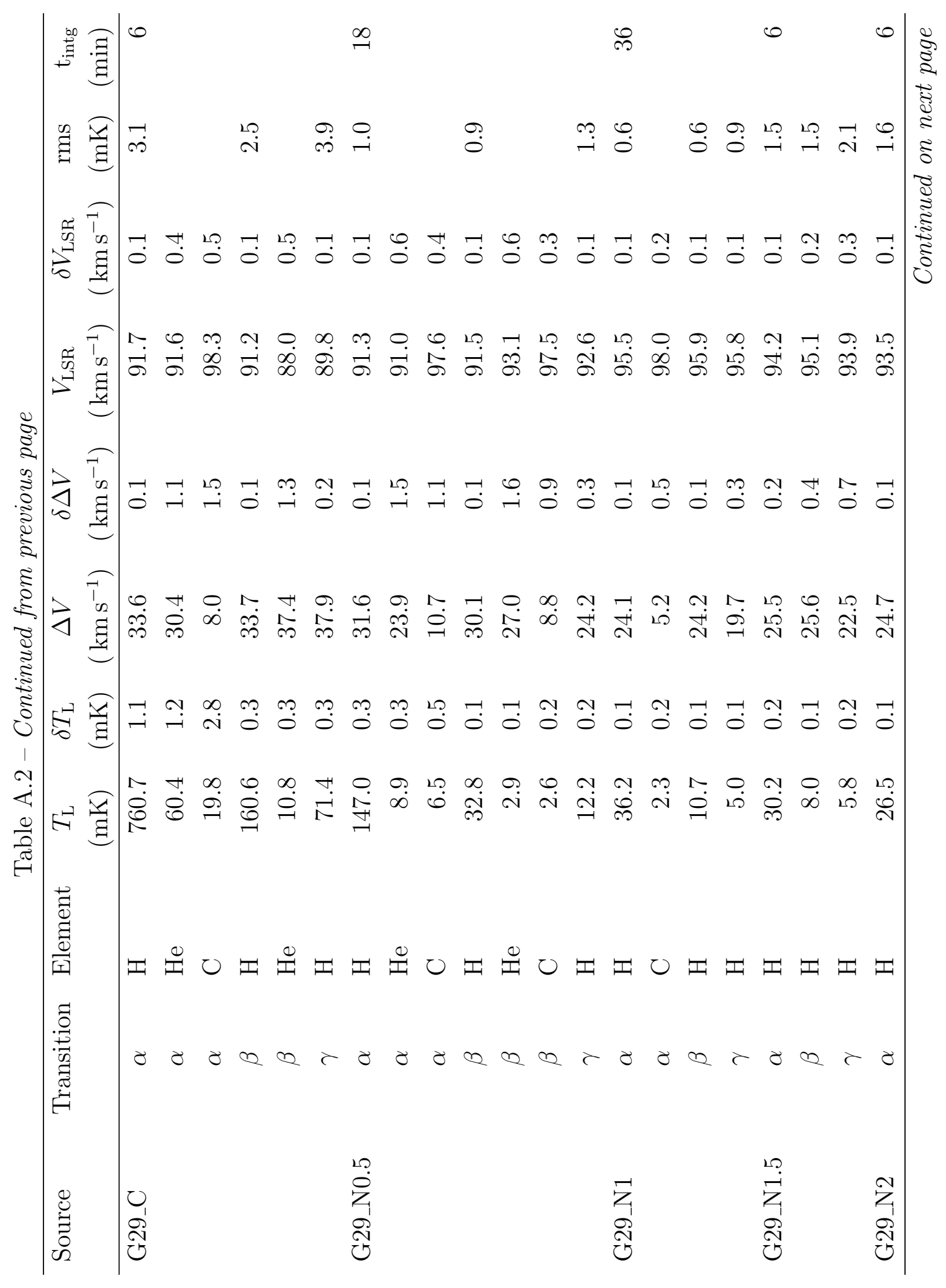




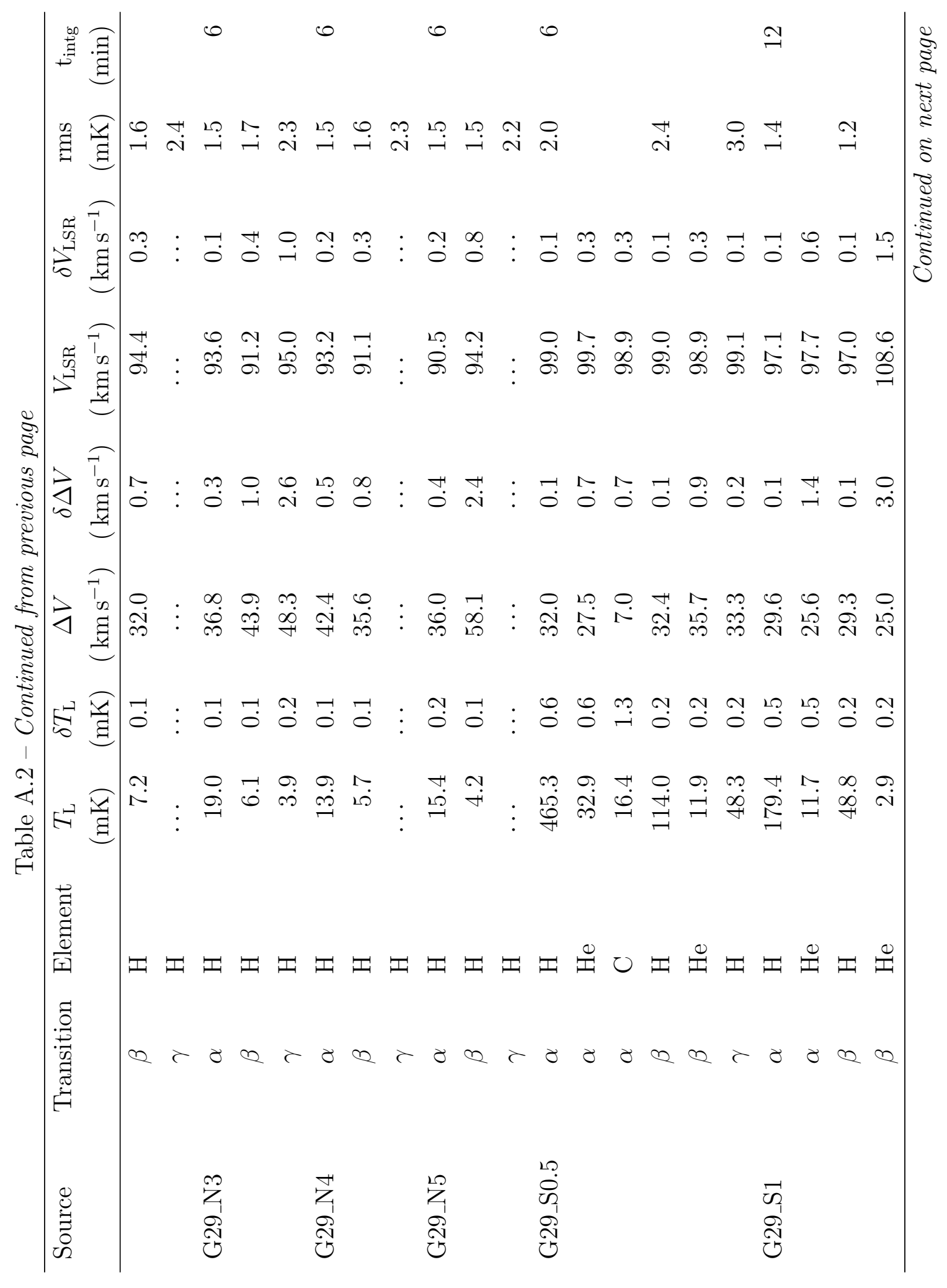




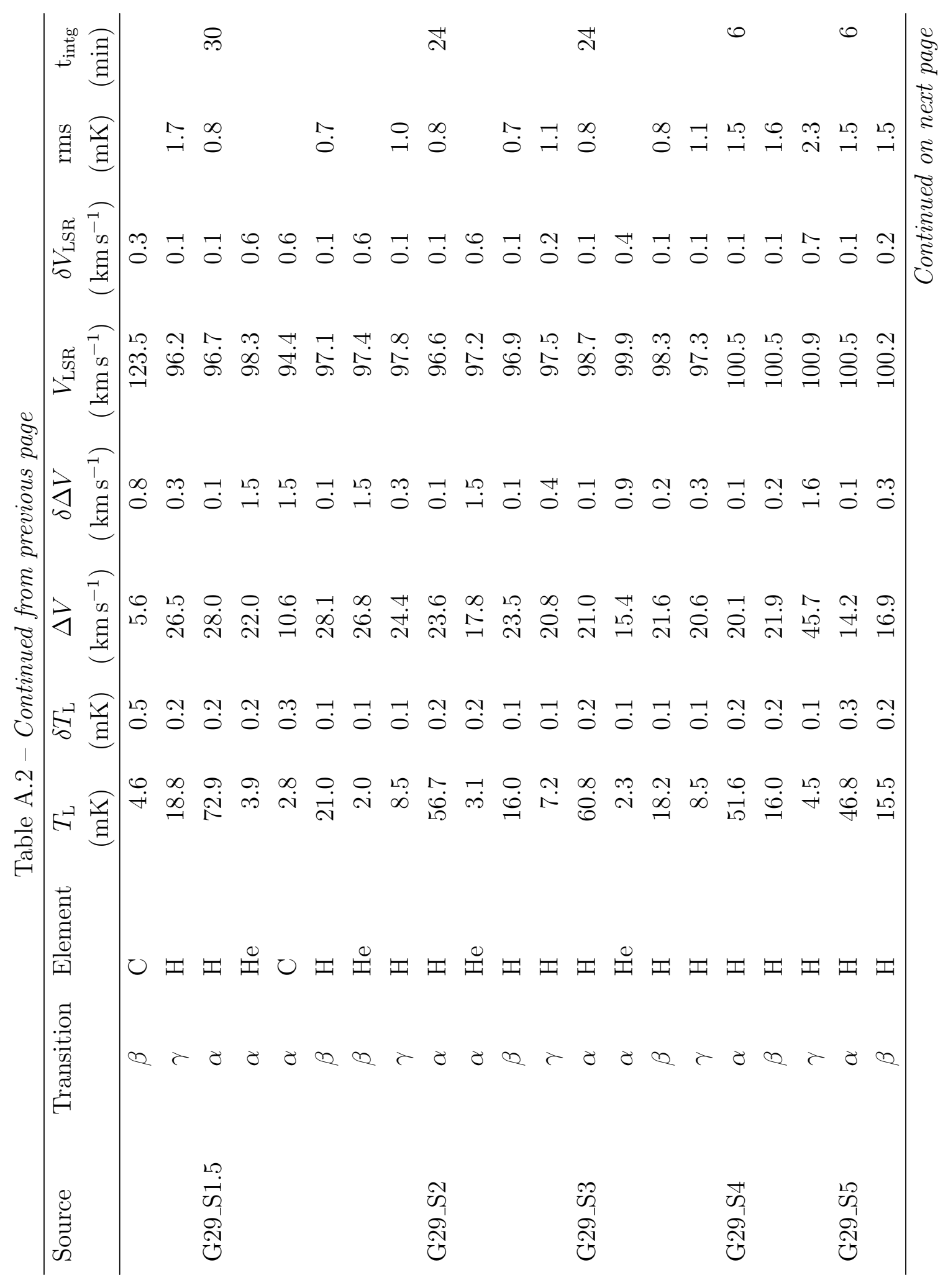




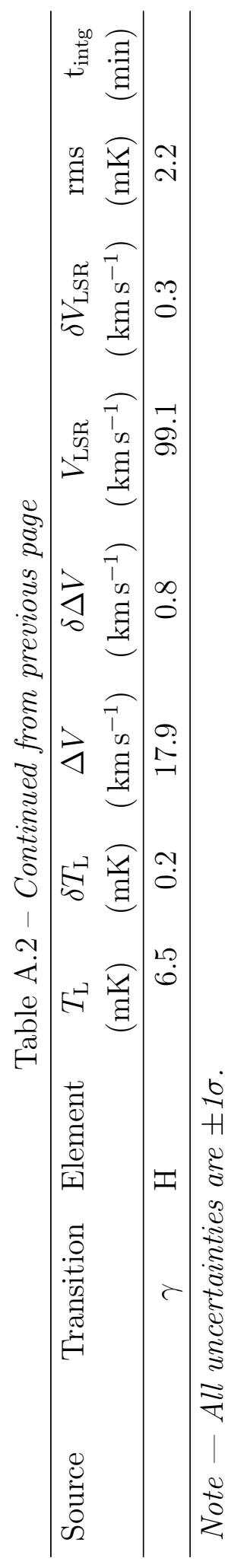


Table A.3: Radio Continuum Temperatures and Source Properties

\begin{tabular}{lcccccc}
\hline Source & $T_{\mathrm{C}}$ & $\delta T_{\mathrm{C}}$ & $\begin{array}{c}T_{\mathrm{e}}^{*} \\
(\mathrm{~K})\end{array}$ & $\begin{array}{c}\delta T_{\mathrm{e}}^{*} \\
(\mathrm{~K})\end{array}$ & $\begin{array}{c}\mathrm{EM} \\
(\mathrm{K})\end{array}$ & $\begin{array}{c}\delta \mathrm{EM} \\
\left(\mathrm{pc} \mathrm{cm}^{-6}\right)\end{array}$ \\
\hline S206_C & 6.90 & 1.00 & 9330 & 1180 & 89030 & 16910 \\
S206_E0.5 & 2.45 & 0.52 & 9550 & 1770 & 31940 & 8890 \\
S206_E1 & 0.61 & 0.11 & 9250 & 1540 & 7760 & 1940 \\
S206_E2 & 0.26 & 0.05 & 9130 & 1690 & 3390 & 940 \\
S206_E3 & 0.12 & 0.03 & $\ldots$ & $\ldots$ & $\ldots$ & $\ldots$ \\
S206_W0.5 & 2.67 & 0.58 & 8860 & 1690 & 33690 & 9650 \\
S206_W1 & 0.74 & 0.14 & 9130 & 1500 & 9420 & 2320 \\
S206_W2 & 0.19 & 0.04 & 10190 & 1820 & 2500 & 670 \\
S206_W3 & 0.05 & 0.02 & $\ldots$ & $\ldots$ & $\ldots$ & $\ldots$ \\
S206_W4 & 0.04 & 0.01 & $\ldots$ & $\ldots$ & $\ldots$ & $\ldots$ \\
& & & & & & \\
S104_C & 2.06 & 0.41 & 7270 & 1280 & 24400 & 6440 \\
S104_N0.5 & 1.42 & 0.28 & 8020 & 1380 & 17510 & 4520 \\
S104_N1 & 0.44 & 0.13 & 7320 & 1950 & 5230 & 2090 \\
S104_N1.5 & 0.10 & 0.02 & $\ldots$ & $\ldots$ & $\ldots$ & $\ldots$ \\
S104_S0.5 & 1.55 & 0.30 & 8010 & 1350 & 19310 & 4870 \\
S104_S1 & 0.38 & 0.09 & 9180 & 2020 & 4950 & 1630 \\
S104_S1.5 & 0.30 & 0.05 & $\ldots$ & $\ldots$ & $\ldots$ & $\ldots$ \\
S104_S2.5 & 0.34 & 0.06 & $\ldots$ & $\ldots$ & $\ldots$ & $\ldots$ \\
& & & & & & \\
G45_C & 13.09 & 1.64 & 7500 & 830 & 158190 & 26290 \\
G45_E0.5 & 3.45 & 0.96 & 7150 & 1750 & 41410 & 15200 \\
G45_E1 & 0.64 & 0.11 & 10320 & 1740 & 8780 & 2220 \\
G45_E2 & 0.32 & 0.22 & $\ldots$ & $\ldots$ & $\ldots$ & $\ldots$ \\
G45_E3 & 0.29 & 0.04 & $\ldots$ & $\ldots$ & $\ldots$ & $\ldots$ \\
G45_W0.5 & 3.34 & 0.65 & 7270 & 1240 & 40140 & 10300 \\
G45_W1 & 0.04 & 0.04 & 1240 & 1080 & 270 & 360 \\
G45_W2 & 0.15 & 0.05 & $\ldots$ & $\ldots$ & $\ldots$ & $\ldots$ \\
G45_W3 & 0.07 & 0.02 & $\ldots$ & $\ldots$ & $\ldots$ & $\ldots$ \\
M16_C & 5.76 & 1.20 & 7420 & 1340 & 69080 & 18750 \\
M16_N0.5 & 5.59 & 1.11 & 8150 & 1410 & 69420 & 17970 \\
\hline & 6.49 & 1.24 & 8520 & 1430 & 81790 & 20530 \\
\hline & & & & & Continued on next page
\end{tabular}


Table A.3 - Continued from previous page

\begin{tabular}{|c|c|c|c|c|c|c|}
\hline Source & $\begin{array}{l}T_{\mathrm{C}} \\
(\mathrm{K})\end{array}$ & $\begin{array}{l}\delta T_{\mathrm{C}} \\
(\mathrm{K})\end{array}$ & $\begin{array}{l}T_{\mathrm{e}}^{*} \\
(\mathrm{~K})\end{array}$ & $\begin{array}{l}\delta T_{\mathrm{e}}^{*} \\
(\mathrm{~K})\end{array}$ & $\begin{array}{c}\mathrm{EM} \\
\left(\mathrm{pc} \mathrm{cm}^{-6}\right)\end{array}$ & $\begin{array}{c}\delta \mathrm{EM} \\
\left(\mathrm{pc} \mathrm{cm}^{-6}\right)\end{array}$ \\
\hline M16_N1.5 & 4.97 & 0.97 & 8900 & 1570 & 64130 & 17010 \\
\hline M16_N2.5 & 2.23 & 0.39 & 8690 & 1360 & 28800 & 6770 \\
\hline M16_N4.5 & 0.19 & 0.06 & $\cdots$ & $\cdots$ & $\cdots$ & $\cdots$ \\
\hline M16_N6.5 & 0.03 & 0.05 & $\cdots$ & $\cdots$ & $\cdots$ & $\cdots$ \\
\hline M16_N8.5 & 0.26 & 0.05 & $\cdots$ & $\cdots$ & $\cdots$ & $\cdots$ \\
\hline M16_N10.5 & 0.31 & 0.06 & $\ldots$ & $\ldots$ & $\cdots$ & $\ldots$ \\
\hline M16_S0.5 & 5.50 & 1.03 & 8070 & 1310 & 68250 & 16640 \\
\hline M16_S1 & 4.06 & 0.82 & 9040 & 1590 & 52460 & 13830 \\
\hline M16_S1.5 & 4.27 & 0.85 & 7790 & 1370 & 51580 & 13600 \\
\hline M16_S2.5 & 3.73 & 0.72 & 8060 & 1360 & 46360 & 11740 \\
\hline M16_S3.5 & 1.23 & 0.25 & 7670 & 1470 & 15270 & 4390 \\
\hline M16_S5.5 & 0.49 & 0.10 & 7810 & 1560 & 6070 & 1820 \\
\hline M16_S9.5 & 0.39 & 0.08 & $\cdots$ & $\cdots$ & $\ldots$ & $\ldots$ \\
\hline M16_S11.5 & 0.29 & 0.04 & $\cdots$ & $\cdots$ & $\ldots$ & $\ldots$ \\
\hline M17_C & 111.11 & 24.07 & 10780 & 2040 & 1500080 & 426320 \\
\hline M17_E1 & 46.96 & 9.81 & 6870 & 1250 & 543540 & 148400 \\
\hline M17_E1.5 & 5.73 & 1.39 & 7610 & 1640 & 69350 & 22360 \\
\hline M17_E2 & 3.72 & 0.82 & 7720 & 1510 & 45360 & 13290 \\
\hline M17_E3 & 2.59 & 0.58 & 7400 & 1470 & 30580 & 9130 \\
\hline M17_E4 & 1.57 & 0.30 & 7840 & 1300 & 19160 & 4780 \\
\hline M17_E5 & 0.32 & 0.07 & 7890 & 1540 & 3760 & 1100 \\
\hline M17_E6 & 0.28 & 0.08 & $\cdots$ & $\cdots$ & $\cdots$ & $\cdots$ \\
\hline M17_E7 & 0.21 & 0.04 & 8720 & 1490 & 2600 & 670 \\
\hline M17_E9 & $\cdots$ & $\cdots$ & $\cdots$ & $\cdots$ & $\cdots$ & $\cdots$ \\
\hline M17_E11 & $\cdots$ & $\cdots$ & $\cdots$ & $\ldots$ & $\ldots$ & $\cdots$ \\
\hline M17_W1 & 14.55 & 5.22 & 6870 & 2140 & 169580 & 79350 \\
\hline M17_W1.5 & 0.90 & 0.23 & 7410 & 1680 & 10850 & 3690 \\
\hline M17_W2 & 0.27 & 0.08 & 5440 & 1370 & 2830 & 1070 \\
\hline M17_W3 & 0.31 & 0.04 & $\cdots$ & $\ldots$ & $\cdots$ & $\cdots$ \\
\hline M17_W4 & 0.29 & 0.05 & $\cdots$ & $\cdots$ & $\ldots$ & $\cdots$ \\
\hline M17_W5 & 0.15 & 0.04 & $\cdots$ & $\cdots$ & $\cdots$ & $\cdots$ \\
\hline
\end{tabular}


Table A.3 - Continued from previous page

\begin{tabular}{|c|c|c|c|c|c|c|}
\hline Source & $\begin{array}{l}T_{\mathrm{C}} \\
(\mathrm{K})\end{array}$ & $\begin{array}{l}\delta T_{\mathrm{C}} \\
(\mathrm{K})\end{array}$ & $\begin{array}{l}T_{\mathrm{e}}^{*} \\
(\mathrm{~K})\end{array}$ & $\begin{array}{l}\delta T_{\mathrm{e}}^{*} \\
(\mathrm{~K})\end{array}$ & $\begin{array}{c}\text { EM } \\
\left(\mathrm{pc} \mathrm{cm}^{-6}\right)\end{array}$ & $\begin{array}{c}\delta \mathrm{EM} \\
\left(\mathrm{pc} \mathrm{cm}^{-6}\right)\end{array}$ \\
\hline Orion_C & 214.56 & 33.39 & 6330 & 860 & 2418330 & 492550 \\
\hline Orion_N1 & 30.89 & 6.57 & 8040 & 1490 & 384950 & 107070 \\
\hline Orion_N2 & 0.94 & 0.25 & $\ldots$ & $\ldots$ & $\ldots$ & $\ldots$ \\
\hline Orion_N3 & 0.07 & 0.02 & $\ldots$ & $\ldots$ & $\ldots$ & $\ldots$ \\
\hline Orion_N4 & 0.05 & 0.01 & $\ldots$ & $\ldots$ & $\ldots$ & $\ldots$ \\
\hline Orion_N5 & 0.05 & 0.01 & $\ldots$ & $\ldots$ & $\ldots$ & $\ldots$ \\
\hline Orion_S1 & 10.90 & 2.40 & 8010 & 1560 & 136530 & 40000 \\
\hline Orion_S2 & 2.48 & 0.52 & 8400 & 1660 & 31280 & 9260 \\
\hline Orion_S3 & 1.32 & 0.25 & 8550 & 1440 & 17030 & 4320 \\
\hline Orion_S4 & 0.34 & 0.07 & $\ldots$ & $\ldots$ & $\ldots$ & $\ldots$ \\
\hline Orion_S5 & 0.08 & 0.03 & $\ldots$ & $\ldots$ & $\ldots$ & $\ldots$ \\
\hline Orion_S6 & 0.14 & 0.03 & $\ldots$ & $\ldots$ & $\ldots$ & $\ldots$ \\
\hline Orion_S7 & 0.09 & 0.02 & $\ldots$ & $\ldots$ & $\ldots$ & $\ldots$ \\
\hline Orion_S9 & 0.01 & 0.01 & $\ldots$ & $\ldots$ & $\ldots$ & $\ldots$ \\
\hline Orion_S11 & $\cdots$ & $\cdots$ & $\cdots$ & $\cdots$ & $\cdots$ & $\cdots$ \\
\hline N49_C & 1.90 & 0.32 & 6250 & 920 & 22120 & 4890 \\
\hline N49_E0.5 & 0.67 & 0.15 & 8100 & 1720 & 8990 & 2870 \\
\hline N49_E1 & 0.39 & 0.06 & $\ldots$ & $\ldots$ & $\ldots$ & $\ldots$ \\
\hline N49_E2 & 0.47 & 0.07 & $\ldots$ & $\ldots$ & $\ldots$ & $\ldots$ \\
\hline N49_W0.5 & 0.94 & 0.21 & 7330 & 1580 & 11750 & 3800 \\
\hline N49_W1 & 0.45 & 0.13 & $\ldots$ & $\ldots$ & $\ldots$ & $\ldots$ \\
\hline N49_W2 & 0.35 & 0.10 & $\ldots$ & $\ldots$ & $\ldots$ & $\cdots$ \\
\hline G29_C & 12.94 & 1.46 & 6490 & 680 & 148100 & 23140 \\
\hline G29_N0.5 & 2.12 & 0.68 & 6050 & 1720 & 24240 & 10340 \\
\hline G29_N1 & 0.45 & 0.09 & 6890 & 1340 & 5530 & 1620 \\
\hline G29_N1.5 & 0.40 & 0.08 & $\ldots$ & $\cdots$ & $\cdots$ & $\cdots$ \\
\hline G29_N2 & 0.61 & 0.07 & $\cdots$ & $\cdots$ & $\ldots$ & $\cdots$ \\
\hline G29_N3 & 0.70 & 0.07 & $\ldots$ & $\cdots$ & $\ldots$ & $\cdots$ \\
\hline G29_N4 & 0.49 & 0.06 & $\cdots$ & $\cdots$ & $\cdots$ & $\cdots$ \\
\hline G29_N5 & 0.37 & 0.07 & $\cdots$ & $\cdots$ & $\cdots$ & $\cdots$ \\
\hline G29_S0.5 & 6.80 & 1.45 & 5980 & 1120 & 76420 & 21480 \\
\hline
\end{tabular}


Table A.3 - Continued from previous page

\begin{tabular}{lcccccc}
\hline Source & $\begin{array}{c}T_{\mathrm{C}} \\
(\mathrm{K})\end{array}$ & $\begin{array}{c}\delta T_{\mathrm{C}} \\
(\mathrm{K})\end{array}$ & $\begin{array}{c}T_{\mathrm{e}}^{*} \\
(\mathrm{~K})\end{array}$ & $\begin{array}{c}\delta T_{\mathrm{e}}^{*} \\
(\mathrm{~K})\end{array}$ & $\begin{array}{c}\mathrm{EM} \\
\left(\mathrm{pc} \mathrm{cm}^{-6}\right)\end{array}$ & $\begin{array}{c}\delta \mathrm{EM} \\
\left(\mathrm{pc} \mathrm{cm}^{-6}\right)\end{array}$ \\
\hline G29_S1 & 2.31 & 0.56 & 5750 & 1270 & 25650 & 8480 \\
G29_S1.5 & 1.07 & 0.20 & 6850 & 1220 & 12820 & 3420 \\
G29_S2 & 0.81 & 0.12 & 7780 & 1230 & 10190 & 2410 \\
G29_S3 & 0.67 & 0.15 & 6980 & 1410 & 8260 & 2510 \\
G29_S4 & 0.41 & 0.15 & $\ldots$ & $\ldots$ & $\ldots$ & $\ldots$ \\
G29_S5 & 0.64 & 0.14 & $\ldots$ & $\ldots$ & $\ldots$ & $\ldots$ \\
\hline
\end{tabular}

Note - All uncertainties are $\pm 1 \sigma$. 

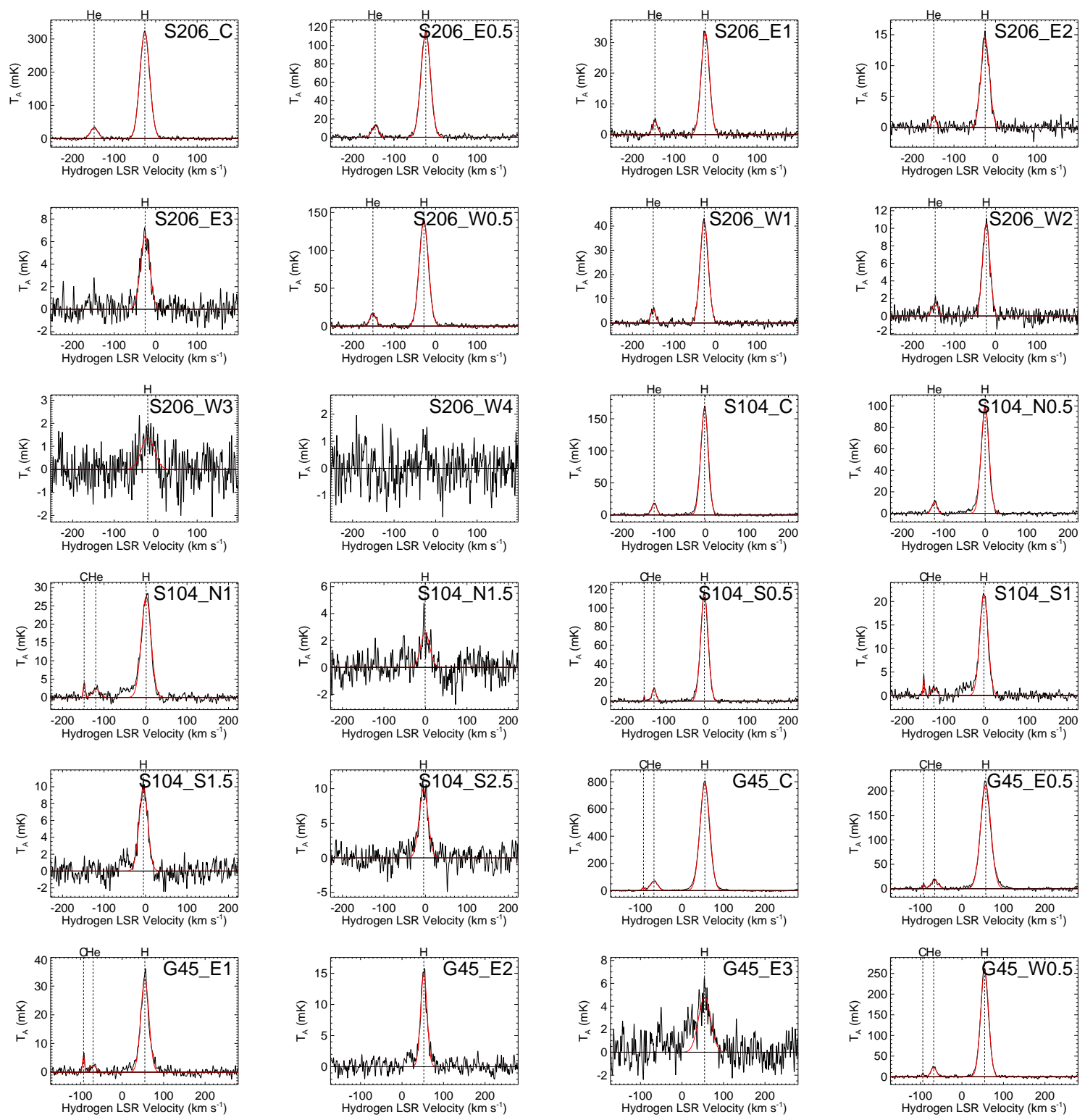

Figure A.1: $\alpha$ RRL spectra of all observed positions, smoothed to a spectral resolution of $1.86 \mathrm{~km} \mathrm{~s}^{-1}$. Plotted is the antenna temperature as a function of hydrogen LSR velocity. The helium and carbon lines are offset from hydrogen by $-124 \mathrm{~km} \mathrm{~s}^{-1}$ and $-149 \mathrm{~km} \mathrm{~s}^{-1}$, respectively. We approximate hydrogen, helium, and carbon emission above the $\mathrm{S} / \mathrm{N}$ threshold defined in $\S 4.3$ with the Gaussian model fits shown in red. The centers of the Gaussian peaks are indicated by dashed vertical lines. The name of the observed position is given in the upper right-hand corner of each plot. 

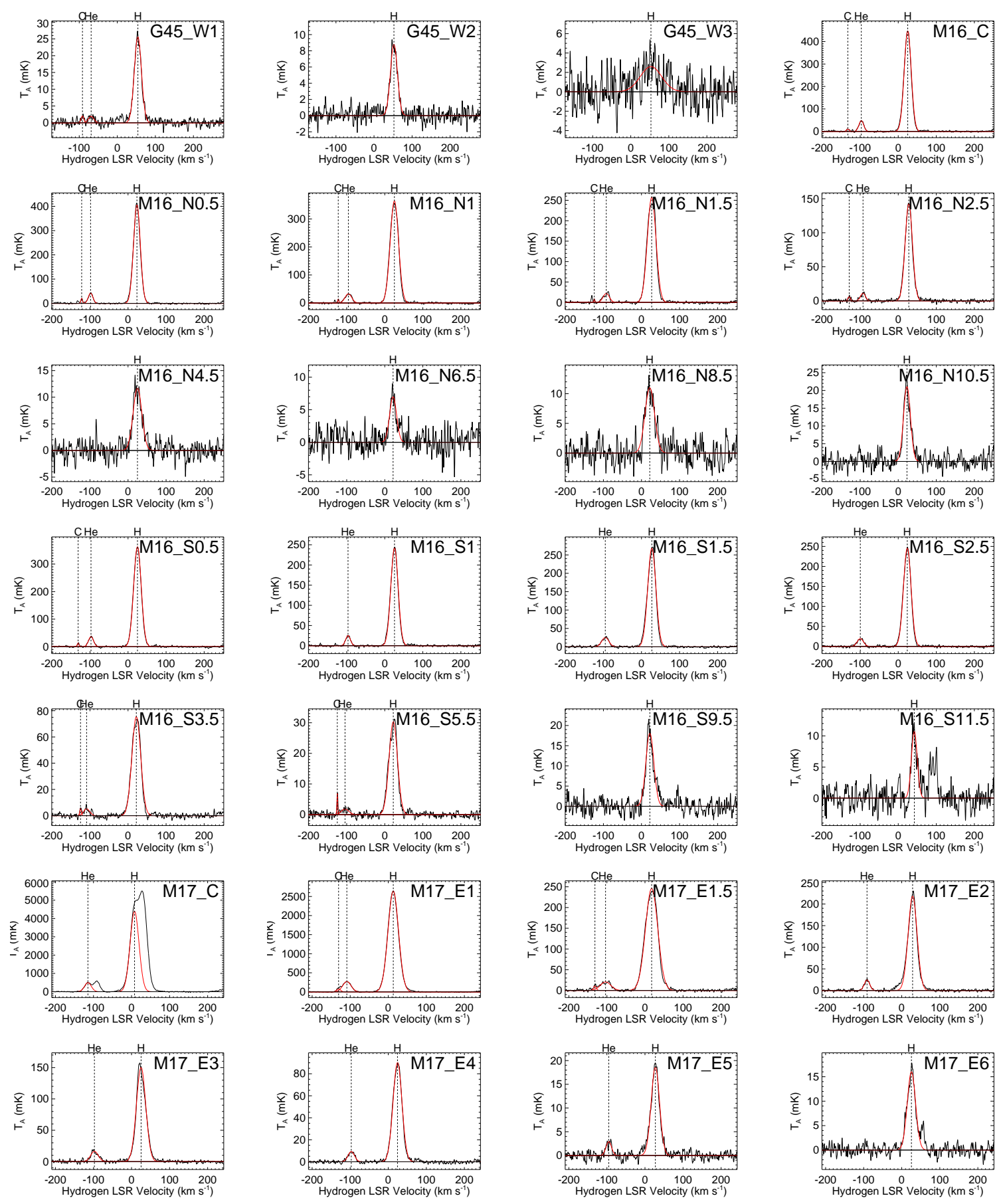

Figure A.1 (cont.) 

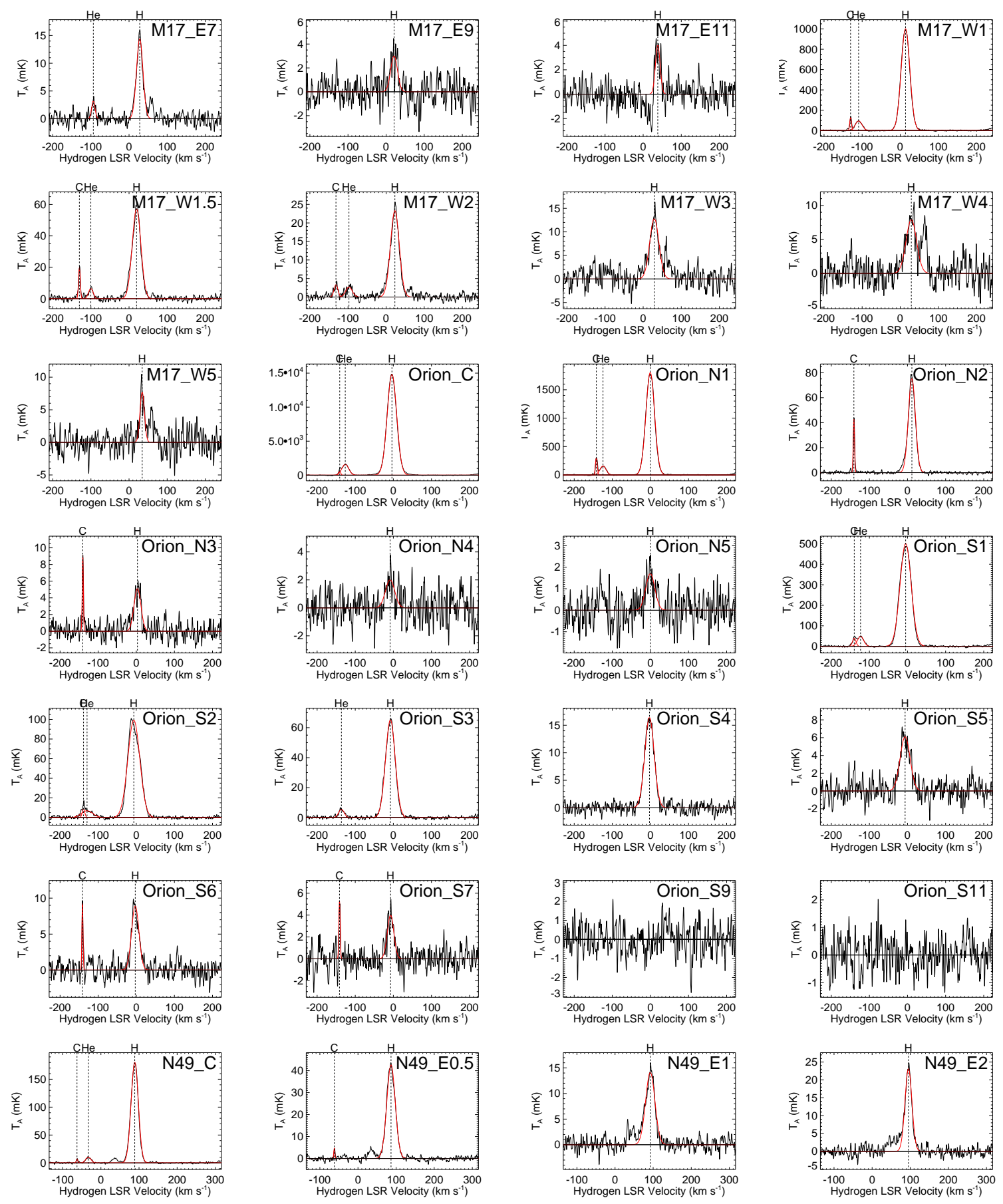

Figure A.1 (cont.) 

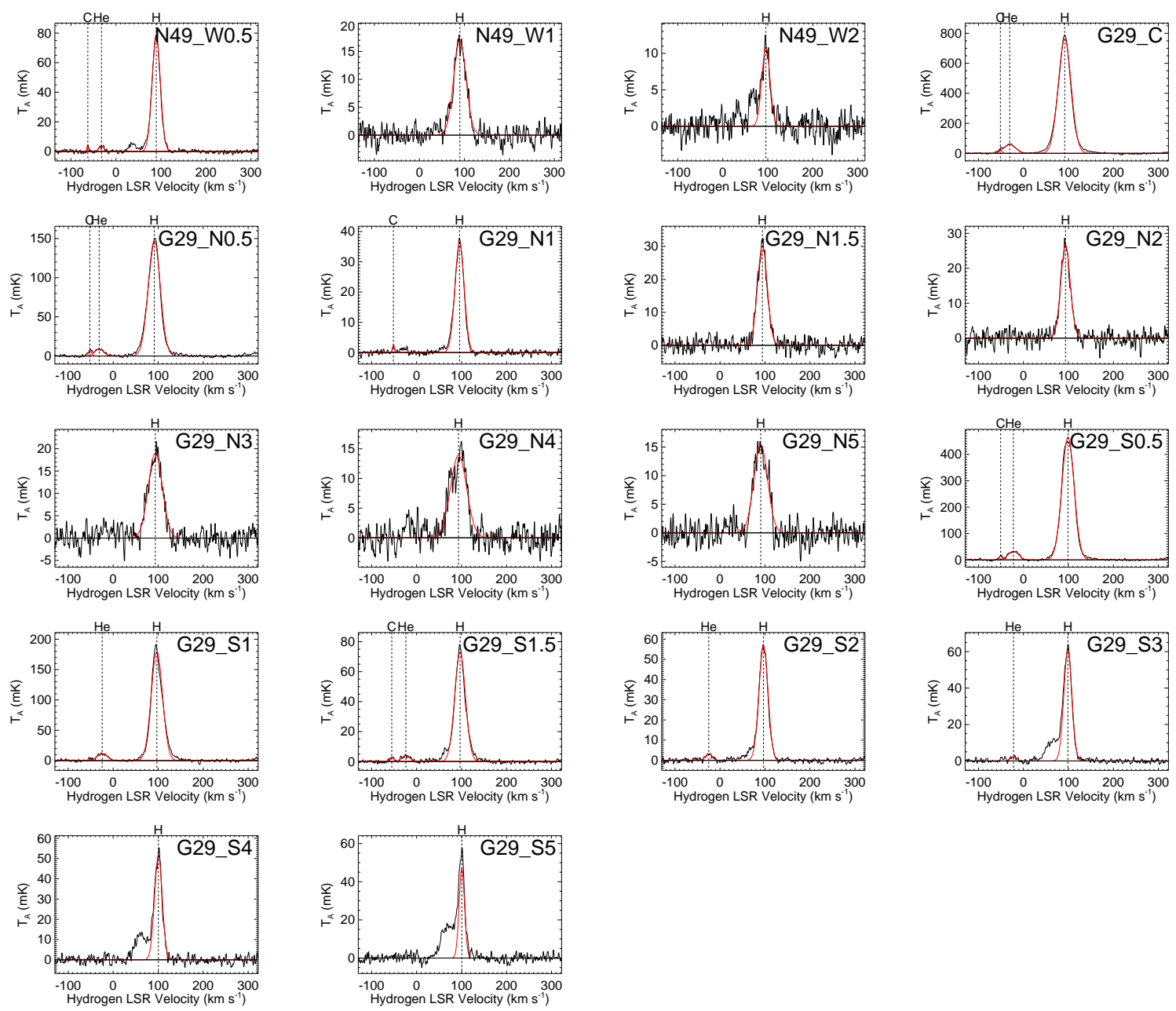

Figure A.1 (cont.) 

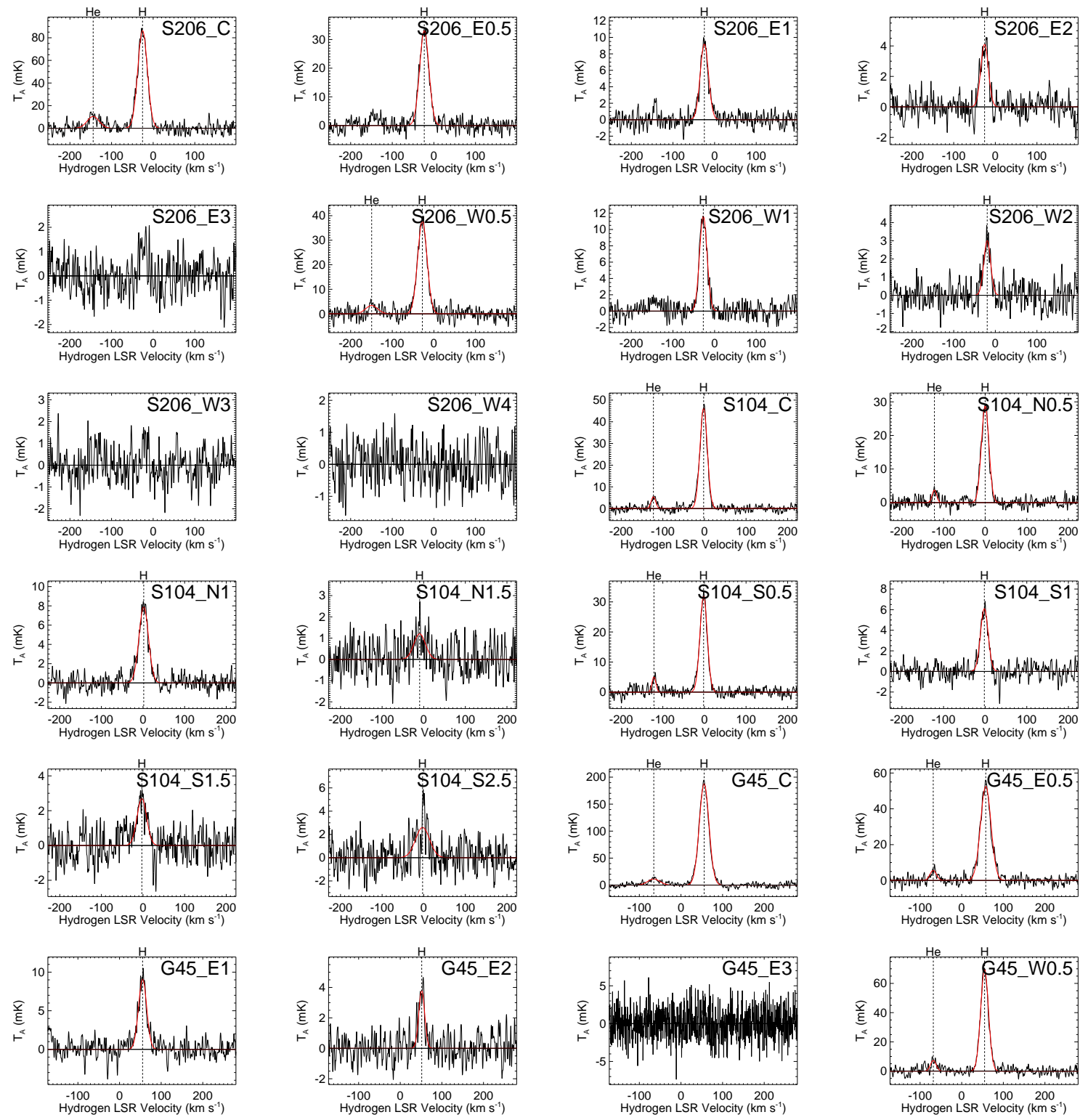

Figure A.2: $\beta$ RRL spectra of all observed positions, smoothed to a spectral resolution of $1.86 \mathrm{~km} \mathrm{~s}^{-1}$. Plotted is the antenna temperature as a function of hydrogen LSR velocity. The helium and carbon lines are offset from hydrogen by $-124 \mathrm{~km} \mathrm{~s}^{-1}$ and $-149 \mathrm{~km} \mathrm{~s}^{-1}$, respectively. We approximate hydrogen, helium, and carbon emission above the $\mathrm{S} / \mathrm{N}$ threshold defined in $\S 4.3$ with the Gaussian model fits shown in red. The centers of the Gaussian peaks are indicated by dashed vertical lines. The name of the observed position is given in the upper right-hand corner of each plot. 

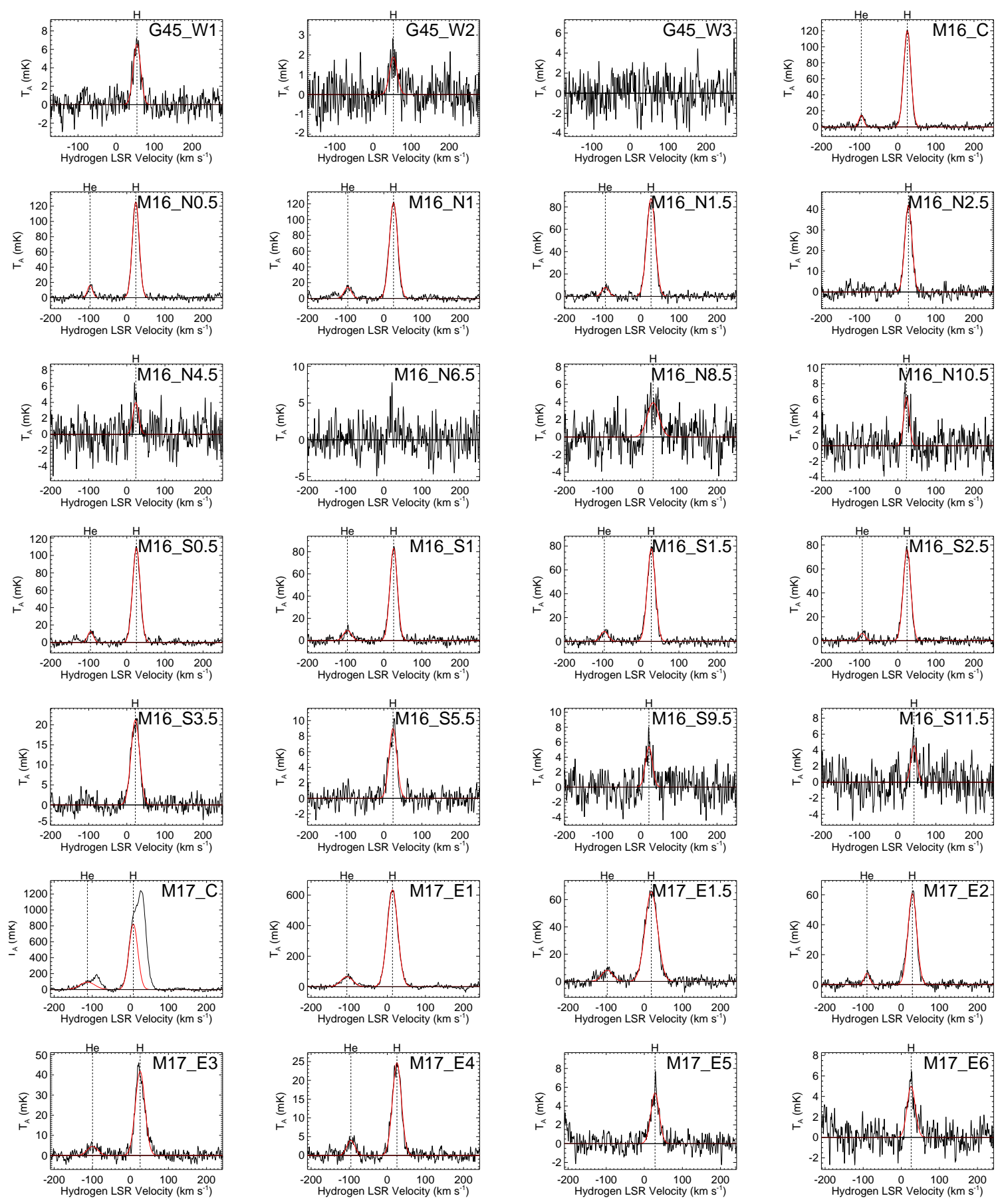

Figure A.2 (cont.) 



Figure A.2 (cont.) 

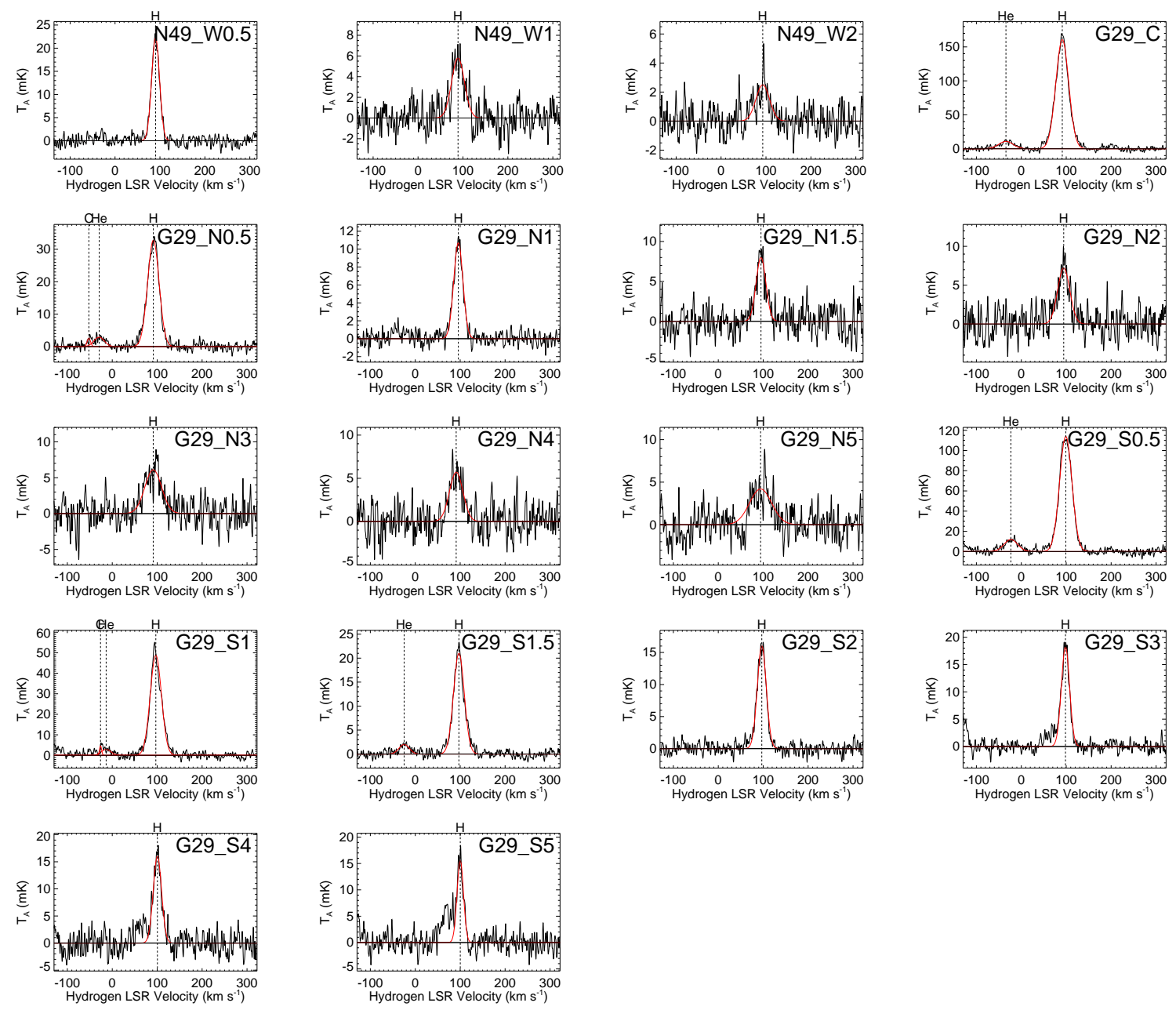

Figure A.2 (cont.) 

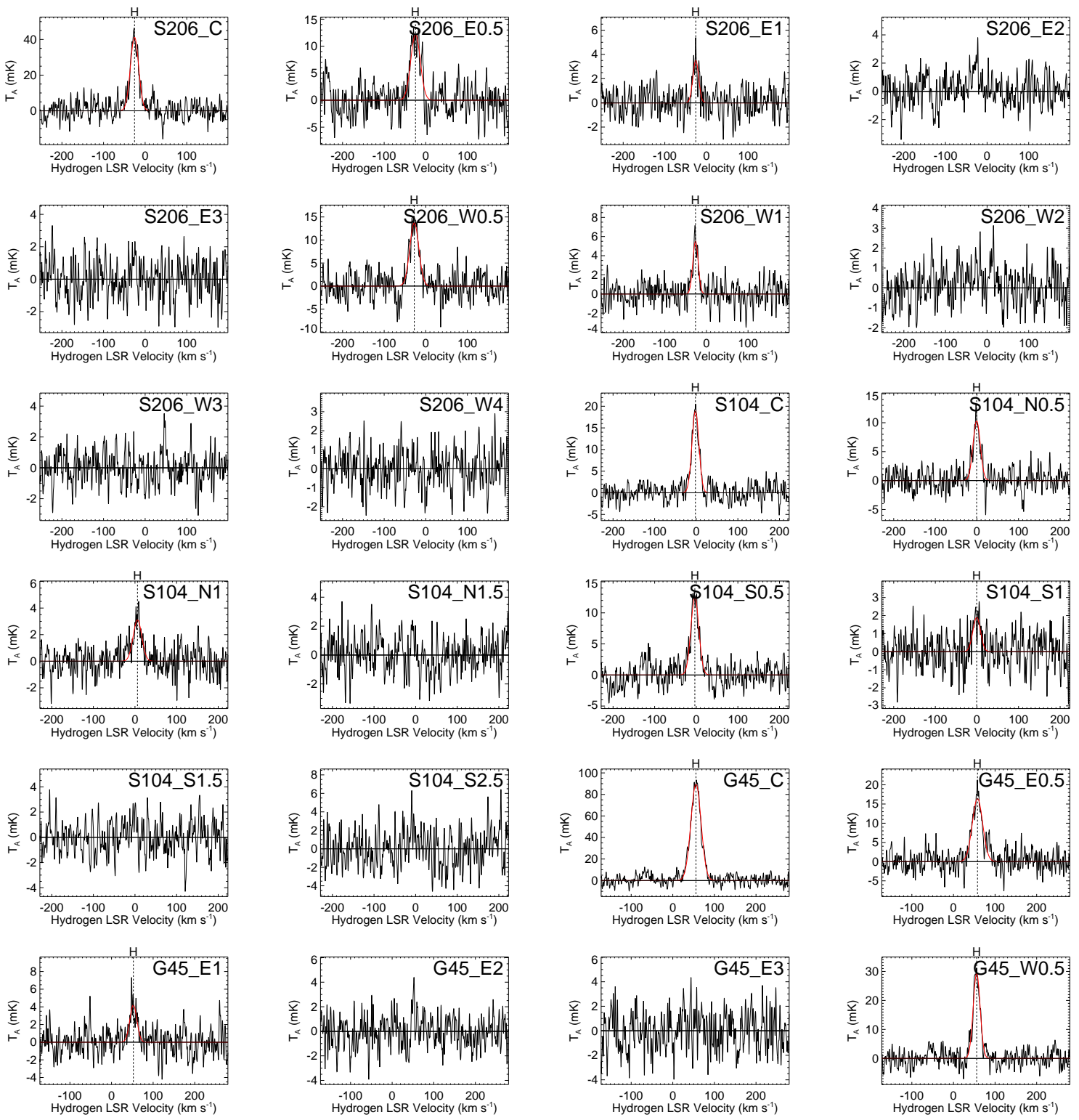

Figure A.3: $\gamma$ RRL spectra of all observed positions, smoothed to a spectral resolution of $1.86 \mathrm{~km} \mathrm{~s}^{-1}$. Plotted is the antenna temperature as a function of hydrogen LSR velocity. The helium and carbon lines are offset from hydrogen by $-124 \mathrm{~km} \mathrm{~s}^{-1}$ and $-149 \mathrm{~km} \mathrm{~s}^{-1}$, respectively. We approximate hydrogen, helium, and carbon emission above the $\mathrm{S} / \mathrm{N}$ threshold defined in $\S 4.3$ with the Gaussian model fits shown in red. The centers of the Gaussian peaks are indicated by dashed vertical lines. The name of the observed position is given in the upper right-hand corner of each plot. 

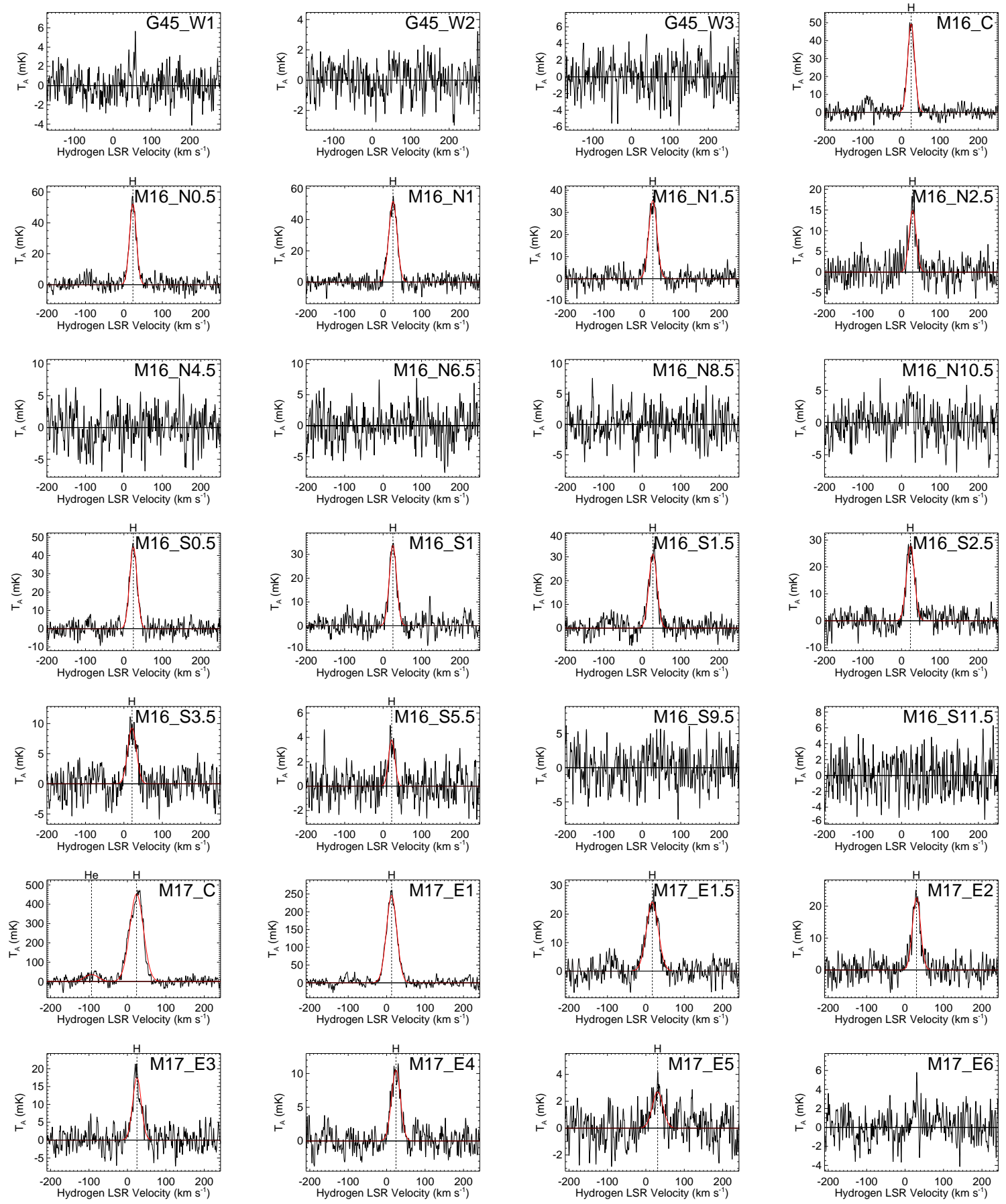

Figure A.3 (cont.) 

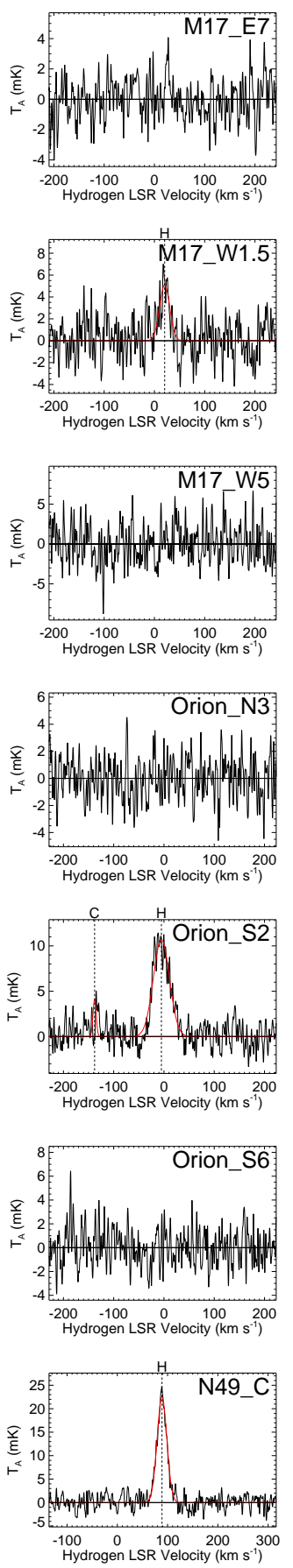
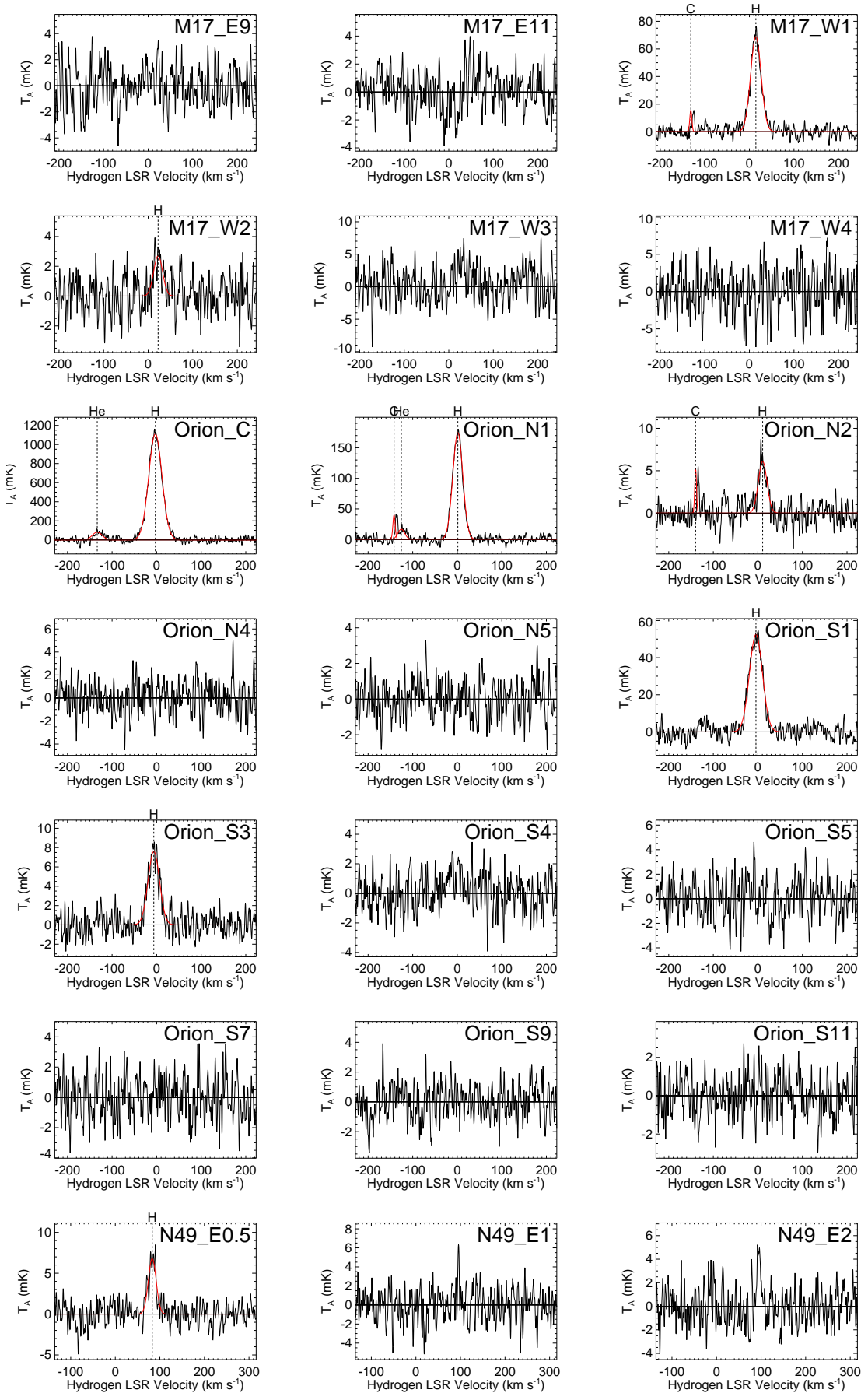

Figure A.3 (cont.) 

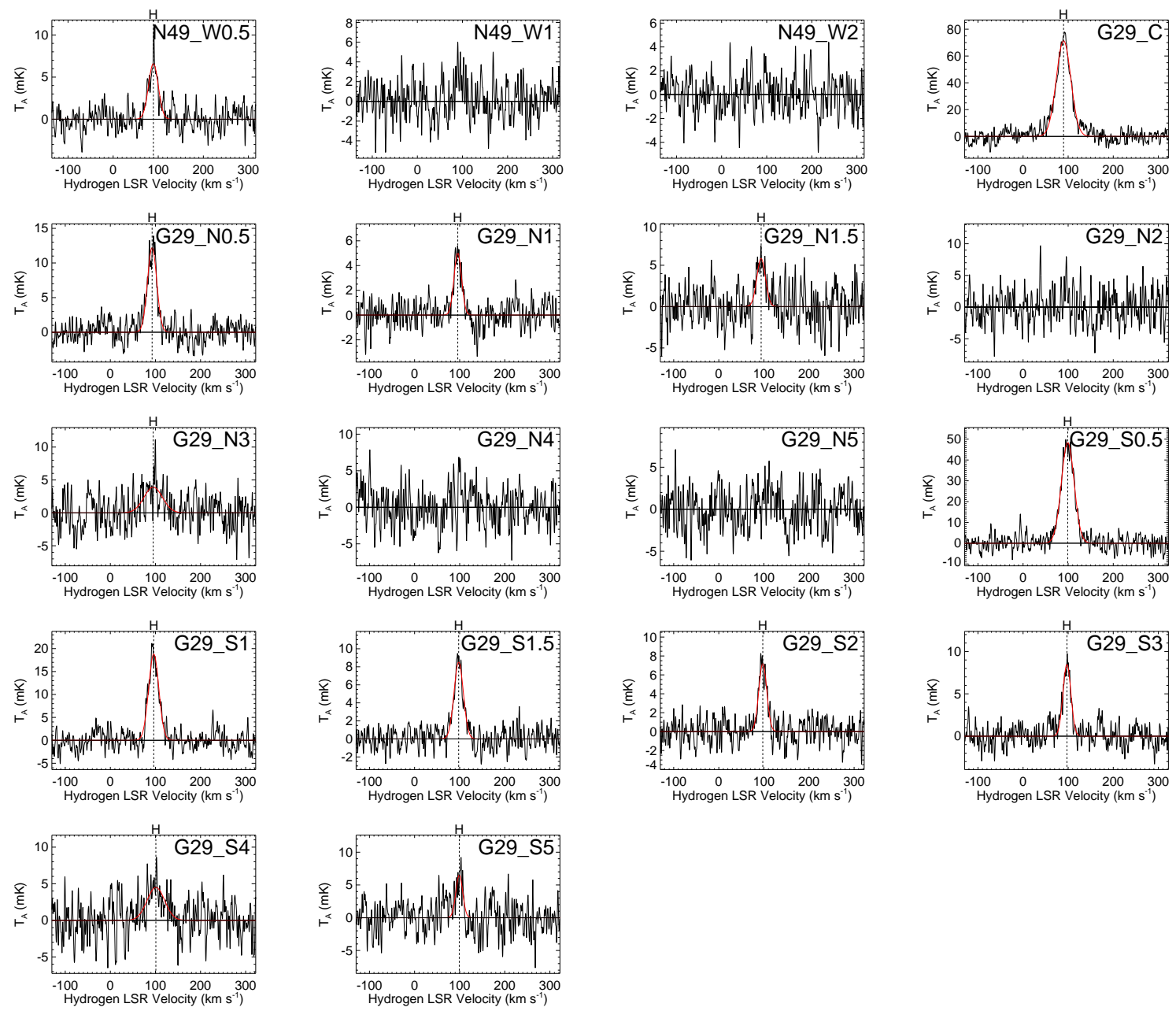

Figure A.3 (cont.) 


\section{Bibliography}

Adler, D. S., Wood, D. O. S., \& Goss, W. M. 1996, ApJ, 471, 871

Afflerbach, A., Churchwell, E., \& Werner, M. W. 1997, ApJ, 478, 190

Alves, M. I. R., Calabretta, M., Davies, R. D., et al. 2015, MNRAS, 450, 2025

Alves, M. I. R., Davies, R. D., Dickinson, C., et al. 2012, MNRAS, 422, 2429

—. 2010, MNRAS, 405, 1654

Anantharamaiah, K. R. 1986, JApA, 7, 131

Anderson, L. D. 2010, PhD thesis, Boston University

Anderson, L. D., Armentrout, W. P., Johnstone, B. M., et al. 2015a, ApJS, 221, 26

Anderson, L. D., Armentrout, W. P., Luisi, M., et al. 2018, ApJS, 234, 33

Anderson, L. D., Bania, T. M., Balser, D. S., \& Rood, R. T. 2011, ApJS, 194, 32

Anderson, L. D., Bania, T. M., Balser, D. S., \& Rood, R. T. 2012a, ApJ, 754, 62

Anderson, L. D., Bania, T. M., Balser, D. S., et al. 2014, ApJS, 212, 1

Anderson, L. D., Hough, L. A., Wenger, T. V., Bania, T. M., \& Balser, D. S. 2015b, ApJ, 810, 42

Anderson, L. D., Zavagno, A., Rodón, J. A., et al. 2010, A\&A, 518, L99

Anderson, L. D., Zavagno, A., Deharveng, L., et al. 2012b, A\&A, 542, 27

Anderson, L. D., Deharveng, L., Zavagno, A., et al. 2015, ApJ, 800, 101

Arthur, S. J., Kurtz, S. E., Franco, J., \& Albarrán, M. Y. 2004, ApJ, 608, 282

Balser, D. S. 2006, AJ, 132, 2326

Balser, D. S., Bania, T. M., Rood, R. T., \& Wilson, T. L. 1999, ApJ, 510, 759 
Balser, D. S., Goss, W. M., \& De Pree, C. G. 2001, AJ, 121, 371

Balser, D. S., Rood, R. T., Bania, T. M., \& Anderson, L. D. 2011, ApJ, 738, 27

Bania, T., Wenger, T., Balser, D., \& Anderson, L. 2014, in tmbidl: TMBIDL v7.1, Zenodo

Bania, T. M., Anderson, L. D., \& Balser, D. S. 2012, ApJ, 759, 96

Bania, T. M., Anderson, L. D., Balser, D. S., \& Rood, R. T. 2010, ApJL, 718, L106

Barentsen, G., Farnhill, H. J., Drew, J. E., et al. 2014, MNRAS, 444, 3230

Becker, R. H., White, R. L., \& Edwards, A. L. 1991, ApJS, 75, 1

Beckman, J. E., Rozas, M., \& Knapen, J. H. 1998, PASA, 15, 83

Beckman, J. E., Rozas, M., Zurita, A., Watson, R. A., \& Knapen, J. H. 2000, AJ, 119,2728

Bell, M. B., Avery, L. W., MacLeod, J. M., \& Vallée, J. P. 2011, Ap\&SS, 333, 377

Benjamin, R. A., Churchwell, E., Babler, B. L., et al. 2003, PASP, 115, 953

Beuther, H., Meidt, S., Schinnerer, E., Paladino, R., \& Leroy, A. 2017, A\&A, 597, A 85

Beuther, H., Tackenberg, J., Linz, H., et al. 2012, A\&A, 538, A11

Binney, J., \& Tremaine, S. 2008, Galactic Dynamics: Second Edition (Princeton University Press)

Bissantz, N., \& Gerhard, O. 2002, MNRAS, 330, 591

Brocklehurst, M., \& Salem, M. 1977, Computer Physics Communications, 13, 39

Broos, P. S., Feigelson, E. D., Townsley, L. K., et al. 2007, ApJS, 169, 353

Burton, W. B., Liszt, H. S., \& Baker, P. L. 1978, ApJL, 219, L67 
Cecil, G., Bland-Hawthorn, J., \& Veilleux, S. 2002, ApJ, 576, 745

Cesaroni, R., Churchwell, E., Hofner, P., Walmsley, C. M., \& Kurtz, S. 1994, A\&A, 288,903

Churchwell, E., Mezger, P. G., \& Huchtmeier, W. 1974, A\&A, 32, 283

Churchwell, E., Babler, B. L., Meade, M. R., et al. 2009, PASP, 121, 213

Ciardi, B., Bianchi, S., \& Ferrara, A. 2002, MNRAS, 331, 463

Compiegne, M., Abergel, A., Verstraete, L., \& Habart, E. 2008, A\&A, 491, 797

Condon, J. J., \& Ransom, S. M. 2016, Essential Radio Astronomy, ed. D. N. Spergel (Princeton University Press)

Cox, D. P., \& Smith, B. W. 1974, ApJL, 189, L105

Dale, J. E., Bonnell, I. A., Clarke, C. J., \& Bate, M. R. 2005, MNRAS, 358, 291

Dame, T. M., Hartmann, D., \& Thaddeus, P. 2001, ApJ, 547, 792

Dame, T. M., Ungerechts, H., Cohen, R. S., et al. 1987, ApJ, 322, 706

Danks, A. C. 1970, A\&A, 9, 175

Dettmar, R.-J. 1990, A\&A, 232, L15

Domgörgen, H., \& Mathis, J. S. 1994, ApJ, 428, 647

Dove, J. B., Shull, J. M., \& Ferrara, A. 2000, ApJ, 531, 846

Draine, B. T. 2011, Physics of the Interstellar and Intergalactic Medium, ed. D. N. Spergel (Princeton)

Drew, J. E., Greimel, R., Irwin, M. J., et al. 2005, MNRAS, 362, 753

Dupree, A. K., \& Goldberg, L. 1970, ARA\&A, 8, 231

Fallscheer, C., Reid, M. A., Di Francesco, J., et al. 2013, ApJ, 773, 102 
Ferguson, A. M. N., Wyse, R. F. G., Gallagher, III, J. S., \& Hunter, D. A. 1996, AJ, 111,2265

Garwood, R. W., \& Dickey, J. M. 1989, ApJ, 338, 841

Georgelin, Y. M., Georgelin, Y. P., \& Roux, S. 1973, A\&A, 25, 337

Ghigo, F., Maddalena, R., Balser, D., \& Langston, G. 2001, GBT Commisioning Memo 10, Tech. rep., National Radio Astronomy Observatory

Giammanco, C., Beckman, J. E., \& Cedrés, B. 2005, A\&A, 438, 599

Gottesman, S. T., \& Gordon, M. A. 1970, ApJL, 162, L93

Green, D. A. 2014, Bulletin of the Astronomical Society of India, 42, 47

Gregory, P. C., \& Condon, J. J. 1991, ApJS, 75, 1011

Guélin, M. 1974, in IAU Symposium, Vol. 60, Galactic Radio Astronomy, ed. F. J. Kerr \& S. C. Simonson, 51

Haddock, F. T., Mayer, C. H., \& Sloanaker, R. M. 1954, ApJ, 119, 456

Haffner, L. M., Reynolds, R. J., \& Tufte, S. L. 1999, ApJ, 523, 223

Haffner, L. M., Dettmar, R.-J., Beckman, J. E., et al. 2009, RvMP, 81, 969

Heiles, C., Koo, B.-C., Levenson, N. A., \& Reach, W. T. 1996, ApJ, 462, 326

Hewish, A., Bell, S. J., Pilkington, J. D. H., Scott, P. F., \& Collins, R. A. 1968, Nature, 217, 709

Hoang-Binh, D. 1972, in Les Spectres des Astres dans l'Infrarouge et les Microondes, $367-370$

Höglund, B., \& Mezger, P. G. 1965, Science, 150, 339

Hollenbach, D. J., \& Tielens, A. G. G. M. 1997a, ARA\&A, 35, 179 
-. 1997b, ARA\&A, 35, 179

Hollenbach, D. J., \& Tielens, A. G. G. M. 1999, RvMP, 71, 173

Hoopes, C. G., \& Walterbos, R. A. M. 2003, ApJ, 586, 902

Howk, J. C., \& Savage, B. D. 2000, AJ, 119, 644

Hoyle, F., \& Ellis, G. R. A. 1963, AJP, 16, 1

Jackson, J. M., Rathborne, J. M., Shah, R. Y., et al. 2006, ApJS, 163, 145

Kalberla, P. M. W., \& Kerp, J. 2009, ARA\&A, 47, 27

Kim, K.-T., \& Koo, B.-C. 2001, ApJ, 549, 979

Kitchin, C. R. 1987, Stars, Nebulae and the Interstellar Medium. Observational Physics and Astrophysics (Bristol: Hilger, 1987)

Knapp, G. R. 1974, AJ, 79, 527

Kolpak, M. A., Jackson, J. M., Bania, T. M., Clemens, D. P., \& Dickey, J. M. 2003, ApJ, 582, 756

Konovalenko, A. A., \& Sodin, L. G. 1981, Nature, 294, 135

Krabbe, A. C., \& Copetti, M. V. F. 2002, A\&A, 387, 295

Kuchar, T. A., \& Bania, T. M. 1994, ApJ, 436, 117

Kurtz, S., Churchwell, E., \& Wood, D. O. S. 1994, ApJS, 91, 659

Lahulla, J. F. 1985, A\&AS, 61, 537

Langer, W. D., Velusamy, T., Goldsmith, P. F., et al. 2017, A\&A, 607, A59

Lebouteiller, V., Bernard-Salas, J., Whelan, D. G., et al. 2011, ApJ, 728, 45

Lenz, D. D., \& Ayres, T. R. 1992, PASP, 104, 1104

Lichten, S. M., Rodriguez, L. F., \& Chaisson, E. J. 1979, ApJ, 229, 524 
Lilley, A. E., Menzel, D. H., Penfield, H., \& Zuckerman, B. 1966, Nature, 209, 468

Liu, B., McIntyre, T., Terzian, Y., et al. 2013, AJ, 146, 80

Luisi, M., Anderson, L. D., Balser, D. S., Bania, T. M., \& Wenger, T. V. 2016, ApJ, 824,125

Luisi, M., Anderson, L. D., Balser, D. S., Wenger, T. V., \& Bania, T. M. 2017, ApJ, 849,117

Luisi, M., Anderson, L. D., Bania, T. M., et al. 2018, PASP, 130, 084101

Luisi, M., Anderson, L. D., Liu, B., Anish Roshi, D., \& Churchwell, E. 2019, ApJS, 241,2

Mac Low, M.-M., \& Ferrara, A. 1999, ApJ, 513, 142

Maddalena, R. J. 2010, Theoretical Ratio of Beam Efficiency to Aperture Efficiency, Tech. rep., National Radio Astronomy Observatory

-. 2012, Modeling the Elevation and Frequency Dependence of Aperture Efficiency for the GBTs Pipeline, Tech. rep., National Radio Astronomy Observatory

Madsen, G. J., Reynolds, R. J., \& Haffner, L. M. 2006, ApJ, 652, 401

Martins, F., Schaerer, D., \& Hillier, D. J. 2005, A\&A, 436, 1049

Mathis, J. S. 1990, ARA\&A, 28, 37

McCall, M. L. 1979, ApJ, 229, 962

McGee, R. X., \& Newton, L. M. 1981, MNRAS, 196, 889

Mehringer, D. M. 1994, ApJS, 91, 713

Mezger, P. G. 1978, A\&A, 70, 565

Mezger, P. G., Burke, B. F., Reifenstein, III, E. C., \& Wilson, T. L. 1967, AJ, 72, 817 
Mezger, P. G., \& Ellis, S. A. 1968, ApJL, 1, 159

Miller, III, W. W., \& Cox, D. P. 1993, ApJL, 417, 579

Moisés, A. P., Damineli, A., Figuerêdo, E., et al. 2011, MNRAS, 411, 705

Moscadelli, L., Reid, M. J., Menten, K. M., et al. 2009, ApJ, 693, 406

Motte, F., Zavagno, A., Bontemps, S., et al. 2010, A\&A, 518, L77

Murray, N., \& Rahman, M. 2010, ApJ, 709, 424

Nguyen Luong, Q., Motte, F., Schuller, F., et al. 2011, A\&A, 529, A41

Norman, C. A., \& Ikeuchi, S. 1989, ApJ, 345, 372

O’Dell, C. R., Kollatschny, W., \& Ferland, G. J. 2017, ApJ, 837, 151

Oey, M. S., \& Kennicutt, R. C. J. 1997, MNRAS, 291, 827

Ojha, D. K., Tamura, M., Nakajima, Y., et al. 2004, ApJ, 616, 1042

Oliveira, V. A., Copetti, M. V. F., \& Krabbe, A. C. 2008, A\&A, 492, 463

Osterbrock, D. 1989, Astrophysics of Gaseous Nebulae and Active Galactic Nuclei (University Science Books)

Pankonin, V., Walmsley, C. M., \& Thum, C. 1980, A\&A, 89, 173

Peimbert, M., Rodriguez, L. F., Bania, T. M., Rood, R. T., \& Wilson, T. L. 1992, ApJ, 395, 484

Pellegrini, E. W., Oey, M. S., Winkler, P. F., et al. 2012, ApJ, 755, 40

Peng, B., Kraus, A., Krichbaum, T. P., \& Witzel, A. 2000, A\&AS, 145, 1

Povich, M. S., Stone, J. M., Churchwell, E., et al. 2007, ApJ, 660, 346

Puga, E., Marn-Franch, A., Najarro, F., et al. 2010, A\&A, 517, A2

Quireza, C., Rood, R. T., Balser, D. S., \& Bania, T. M. 2006, ApJS, 165, 338 
Quireza, C., Rood, R. T., Bania, T. M., Balser, D. S., \& Maciel, W. J. 2006, ApJ, 653,1226

Reid, M. J., Dame, T. M., Menten, K. M., \& Brunthaler, A. 2016, ApJ, 823, 77

Reid, M. J., Menten, K. M., Brunthaler, A., et al. 2014, ApJ, 783, 130

Renaud, F., Bournaud, F., Emsellem, E., et al. 2013, MNRAS, 436, 1836

—. 2015, MNRAS, 454, 3299

Reynolds, R. J. 1971, PhD thesis, University of Wisconsin-Madison

-. 1984, ApJ, 282, 191

-. 1989, ApJL, 339, L29

Reynolds, R. J., Scherb, F., \& Roesler, F. L. 1973, ApJ, 185, 869

Reynolds, R. J., Sterling, N. C., \& Haffner, L. M. 2001, ApJL, 558, L101

Reynolds, R. J., \& Tufte, S. L. 1995, ApJL, 439, L17

Reynolds, R. J., Tufte, S. L., Haffner, L. M., Jaehnig, K., \& Percival, J. W. 1998, PASA, 15, 14

Reynolds, R. J., Tufte, S. L., Kung, D. T., McCullough, P. R., \& Heiles, C. 1995, ApJ, 448, 715

Robitaille, T. P., Churchwell, E., Benjamin, R. A., et al. 2012, A\&A, 545, A39

Roelfsema, P. R., Goss, W. M., \& Mallik, D. C. V. 1992, ApJ, 394, 188

Roman-Duval, J., Jackson, J. M., Heyer, M., et al. 2009, ApJ, 699, 1153

Roshi, D. A., \& Anantharamaiah, K. R. 2001, ApJ, 557, 226

Roshi, D. A., Churchwell, E., \& Anderson, L. D. 2017, ApJ, 838, 144

Roshi, D. A., Kantharia, N. G., \& Anantharamaiah, K. R. 2002, A\&A, 391, 1097 
Roshi, D. A., Plunkett, A., Rosero, V., \& Sravani, V. 2012, ApJ, 749, 49

Rossa, J., \& Dettmar, R.-J. 2003, A\&A, 406, 493

Rossa, J., Dettmar, R.-J., Walterbos, R. A. M., \& Norman, C. A. 2004, AJ, 128, 674

Rozas, M., Castaneda, H. O., \& Beckman, J. E. 1998, A\&A, 330, 873

Rubin, R. H. 1968, ApJ, 154, 391

—. 1985, ApJS, 57, 349

Rubin, R. H., Martin, P. G., Dufour, R. J., et al. 2003, MNRAS, 340, 362

Salem, M., \& Brocklehurst, M. 1979, ApJS, 39, 633

Sellwood, J. A., \& Sánchez, R. Z. 2010, MNRAS, 404, 1733

Shaver, P. A. 1980, A\&A, 90, 34

Shaver, P. A., \& Wilson, T. L. 1979, A\&A, 79, 312

Smith, L. F., Biermann, P., \& Mezger, P. G. 1978, A\&A, 66, 65

Sota, A., Maíz Apellániz, J., Walborn, N. R., et al. 2011, ApJS, 193, 24

Stil, J. M., Taylor, A. R., Dickey, J. M., et al. 2006, AJ, 132, 1158

Stock, D. J., \& Peeters, E. 2017, ApJ, 837, 129

Strickland, D. K., Heckman, T. M., Colbert, E. J. M., Hoopes, C. G., \& Weaver, K. A. 2004, ApJS, 151, 193

Strömgren, B. 1939, ApJ, 89, 526

Struve, O., \& Elvey, C. T. 1938, ApJ, 88, 364

Ter Haar, D. 1946, MNRAS, 106, 283

Terebey, S., Fich, M., Taylor, R., Cao, Y., \& Hancock, T. 2003, ApJ, 590, 906 
Thum, C., Mezger, P. G., \& Pankonin, V. 1980, A\&A, 87, 269

Voit, G. M. 1992, MNRAS, 258, 841

Walmsley, C. M., \& Watson, W. D. 1982, ApJ, 260, 317

Walter, D. K. 1994, PASP, 106, 106

Watson, C., Povich, M. S., Churchwell, E. B., et al. 2008, ApJ, 681, 1341

Weber, J. A., Pauldrach, A. W. A., \& Hoffmann, T. L. 2019, A\&A, 622, A115

Weilbacher, P. M., Monreal-Ibero, A., Kollatschny, W., et al. 2015, A\&A, 582, A114

Wenger, T. V., Bania, T. M., Balser, D. S., \& Anderson, L. D. 2013, ApJ, 764, 34

Wilson, T. L., Bania, T. M., \& Balser, D. S. 2015, ApJ, 812, 45

Wood, D. O. S., \& Churchwell, E. 1989, ApJS, 69, 831

Wood, K., Hill, A. S., Joung, M. R., et al. 2010, ApJ, 721, 1397

Wood, K., \& Loeb, A. 2000, ApJ, 545, 86

Wood, K., \& Mathis, J. S. 2004, MNRAS, 353, 1126

Wright, E. L., Eisenhardt, P. R. M., Mainzer, A. K., et al. 2010, AJ, 140, 1868

Zhang, B., Moscadelli, L., Sato, M., et al. 2014, ApJ, 781, 89

Zuckerman, B., Palmer, P., Penfield, H., \& Lilley, A. E. 1967, ApJL, 149, L61

Zurita, A., Beckman, J. E., Rozas, M., \& Ryder, S. 2002, A\&A, 386, 801

Zurita, A., Rozas, M., \& Beckman, J. E. 2000, A\&A, 363, 9 